\title{
Ultrasonic Localization of a Quadrotor using a Portable Beacon
}

by

\author{
John O'Keefe \\ A Thesis submitted to \\ the Faculty of Graduate Studies and Research \\ in partial fulfilment of \\ the requirements for the degree of \\ Master of Applied Science \\ in
}

\author{
Aerospace Engineering \\ Department of Mechanical and Aerospace Engineering \\ Carleton University \\ Ottawa, Ontario, Canada
}

October 2019

Copyright (C)

2019 - John O’Keefe 
The undersigned recommend to

the Faculty of Graduate Studies and Research

acceptance of the Thesis

\title{
Ultrasonic Localization of a Quadrotor using a Portable Beacon
}

\author{
Submitted by John O'Keefe \\ in partial fulfilment of the requirements for the degree of \\ Master of Science
}

Dr. J. Etele, Supervisor

Dr. R. Miller, Department Chair

Carleton University

2019 


\section{Abstract}

This thesis presents a method for localization of a quadrotor using ultrasound with five receiving nodes and a portable beacon. The time of flight is measured from when the ultrasonic signal is produced to when it is received and triggered through threshold detection. Two different lateration algorithms are explored to determine the position, the analytical trilateration and linear least squares method. The linear least squares algorithm outperforms the analytical trilateration method for static position testing and GPS-denied hover. A new approach named the combined lateration algorithm combines these two methods and has the best stationary standard deviation with $8.0 \mathrm{~cm}$ within a one metre radius at a height of $1.77 \mathrm{~m}$. It calculates a successful measurement $93 \%$ of the time, which provides an average update rate of $9.3 \mathrm{~Hz}$. Successful autonomous hover and landing is performed using a stationary and moving beacon within a GPS-denied environment. 


\section{Acknowledgments}

There are so many people who have helped me through my journey at Carleton University. To start, I would like to thank Carleton University for providing a fantastic learning experience through my Undergraduate and masters degree.

A massive thanks goes to Professor Jason Etele who has been an excellent mentor throughout my degree. I'm still amazed at the ability to break down a problem to its roots and solve it methodically. I always enjoy the presentation dry runs where you completely reorganize presentations in a clearer manner. I am thankful for the research opportunity you provided through a project I am genuinely interested in with the ability to grow and learn new skills. The opportunity you provided to travel to San Diego and present at AIAA was phenomenal. The rowing chats are fun and have given me a benchmark to strive for when I take up rowing.

A huge thanks goes to Ali Raza for the continuous support and help throughout my degree. From the very beginning you were excited to explain everything about TARA and your patience was incredible. You were always ready and willing to video chat when I was running into problems and provided great insight. Your experience with controllers was incredibly helpful while tuning my own PID controllers. You provided me with a great start with access to TARA 1 and a preliminary version of the ultrasonic localization system.

Many thanks to Mark Sutherland who designed and built TARA 3 which I used for most of my testing. Thanks for providing me ideas on the first steps on integrating 
the ultrasonic localization system on TARA 3.

A special thanks goes to the RUAS group, more specifically Brian Lemky and Craig Osborn. They developed the idea of the ultrasonic localization and provided a first version of the system.

A massive thanks to Nagui Mikhail, who without I'd still be playing around on a breadboard. You were a massive help with developing my first PCB and troubleshooting the issues along the way. Beforehand I didn't even know how to solder or even what a PCB stood for.

A huge thanks to my family for their continuous support through this journey. I'd like to thank my mom for her excellent video skills. I'd also like to thank my mom and dad for always being ready to drive me to and from school for testing.

I'd like to thank everyone from my office and my friends for the good times and helpful conversations. A special thanks goes to Brendan Ooi Xin-Zhi and Salman Shafi who are always ready to chat, help me test or help me with anything. I'd also like to thank Pao for helping me test and research.

Thank you to Steve Truttman for providing all the tools I would ever need. Thanks to David Raude who would help me dispose of my expired batteries. A big thanks to Stephan Biljan for printing my parts and doing it in a rush after I crashed. Thanks to James Cann who helped me investigate cross correlation techniques. Thanks to Alex Proctor and Kevin Sangster who are always available to chat and let me make small modifications in the shop even if they're busy.

Thanks to everyone in the MAE Office, including Janet Perras, Dan Sawyer, and Allyson Fitch for always allowing me to book the meeting room and collecting my packages. I'd like to thank Professor Irani for meeting with to discuss the implementation of linear least squares and an extended Kalman filter. 


\section{Table of Contents}

Abstract $\quad$ iii

Acknowledgments $\quad$ iv

Table of Contents $\quad$ vi

List of Tables $\quad$ xi

List of Figures $\quad$ xii

List of Acronyms $\quad$ xvi

List of Symbols $\quad$ xx

1 Introduction 1

1.1 Overview and Motivation . . . . . . . . . . . . . . . . 1

1.2 Problem Statement . . . . . . . . . . . . . . . . . . . 3

1.3 Organization of Thesis . . . . . . . . . . . . . . 3

2 Literature Review $\quad 5$

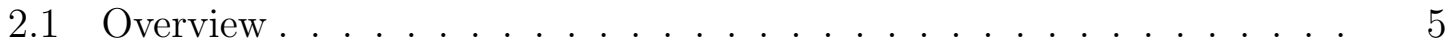

2.2 Localization Methods . . . . . . . . . . . . . 6

2.2.1 Global Navigation Satellite System . . . . . . . . . . 6

2.2.2 Vision-Based Localization _. . . . . . . . . . . . . . . . . . 9 
2.2 .3 Ultrasound Localization . . . . . . . . . . . . . . . . . 12

2.2 .4 Ultra-wideband Localization . . . . . . . . . . . . . . . . . 13

2.2 .5 Infrared Localization . . . . . . . . . . . . . . . . 13

2.2 .6 WiFi Localization . . . . . . . . . . . . . . . . . . . 13

2.2 .7 Bluetooth Localization . . . . . . . . . . . . . . . . . . 14

2.2.8 Radio Frequency Identification Device Localization . . . . . . 14

2.3 Ultrasonic Ranging Techniques . . . . . . . . . . . . . . . . . 14

2.3 .1 Received Signal Strength . . . . . . . . . . . . . . . . . 15

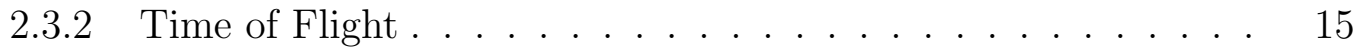

2.3.3 Time Difference of Arrival . . . . . . . . . . . . . . 16

2.3.4 Return Time of Flight . . . . . . . . . . . . . . . . 16

2.3.5 Phase of Arrival . . . . . . . . . . . . . . . . . . . . 17

2.4 Ultrasonic ToF Detection Methods . . . . . . . . . . . . . 17

2.4.1 Threshold . . . . . . . . . . . . . . . . . . . . . . . . 18

2.4 .2 Correlation Techniques . . . . . . . . . . . . . . . . . . . 19

2.4 .3 Envelope . . . . . . . . . . . . . . . . . . . . 20

2.5 Ultrasonic ToF Lateration Techniques . . . . . . . . . . . . . 20

2.5.1 Analytical Trilateration _. . . . . . . . . . . . . . . 21

2.5.2 Linear Least Squares . . . . . . . . . . . . . . . . . . . 22

2.5 .3 Nonlinear Least Squares . . . . . . . . . . . . . . . . . 23

2.5.4 Extended Kalman Filter . . . . . . . . . . . . . . . . . 23

2.5.5 Subset Generation . . . . . . . . . . . . . . 23

2.5.6 Angle of Arrival . . . . . . . . . . . . . . . . . . . . 24

2.6 Lateration Experimental Work . . . . . . . . . . . . . . . 24

2.6 .1 Early Work . . . . . . . . . . . . . . . . . . . 24

2.6 .2 Ultrasonic Localization . . . . . . . . . . . . . . . . . 25

2.6.3 Other Localization Methods . . . . . . . . . . . . . . . 28 
2.6 .4 Commercial Systems . . . . . . . . . . . . . . . . . . 30

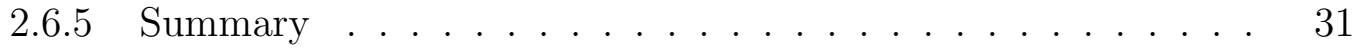

3 Flight Vehicle $\quad 38$

3.1 Sensors . . . . . . . . . . . . . . . . . . . . . . . . . . . . . . . 39

3.1 .1 Pixhawk . . . . . . . . . . . . . . . . 39

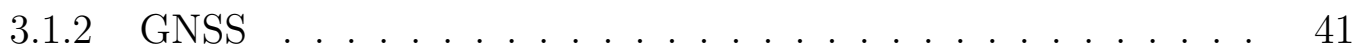

3.2 Software . . . . . . . . . . . . . . . . . . . . . . 41

3.2 .1 Arducopter . . . . . . . . . . . . . . . . . 42

3.2.2 Raspberry Pi Position Controller . . . . . . . . . . . . . . 43

3.2 .3 Control . . . . . . . . . . . . . . . . . . . 46

4 Ultrasonic Localization System $\quad 53$

4.1 Ultrasonic Time of Flight Localization . . . . . . . . . . . . . 54

4.2 Hardware . . . . . . . . . . . . . . . . . . . . . 58

4.2 .1 Piezo Electric Transducer _ . . . . . . . . . . . . . . . . 60

$4.2 .2 \quad$ Beacon . . . . . . . . . . . . . . . . . . . . . . 61

4.2 .3 Receiver Hardware . . . . . . . . . . . . . . . . . . 63

4.2.4 PSoC Digital Hardware . . . . . . . . . . . . . . . . . 67

4.2.5 Theoretical Detection Distance _. . . . . . . . . . . 72

4.3 Main Control Loop . . . . . . . . . . . . . . . . . . . . . . 74

4.3.1 Analytical Method . . . . . . . . . . . . . . . . . . 74

4.3 .2 Linear Least Squares Method _. . . . . . . . . . . . . . 79

4.3 .3 Dilution of Precision . . . . . . . . . . . . . . 85

4.3 .4 Kalman Filter . . . . . . . . . . . . . . . . . . . . . . . . 93

4.3 .5 Algorithms . . . . . . . . . . . . . . . . 96 
5.1 Lateration Algorithm . . . . . . . . . . . . . . . . . . . . 102

5.1.1 Accuracy Assessment . . . . . . . . . . . . . . . 102

5.1 .2 GPS-Denied Hover Flight Testing . . . . . . . . . . . . . . . . 107

5.2 Combined Lateration Algorithm . . . . . . . . . . . . . . . . . . 111

5.2.1 Accuracy Assessment . . . . . . . . . . . . . . . 114

5.2.2 GPS-Denied Hover Flight Testing . . . . . . . . . . . . . 115

5.2 .3 GPS-Denied Landing . . . . . . . . . . . . . . . . . . 117

5.3 GPS Comparison . . . . . . . . . . . . . . . . 121

5.3.1 Stationary Testing . . . . . . . . . . . . . . . 121

5.3.2 Hover Testing . . . . . . . . . . . . . . . . . . 122

5.4 Portable Beacon . . . . . . . . . . . . . . . . 125

5.4 Hover ......................... 125

5.4 Landing ..................... . . 127

6 Conclusion $\quad 130$

6.1 Summary of Contributions . . . . . . . . . . . . . . . . . 131

6.2 Future Research . . . . . . . . . . . . . . . . . . . . . . 132

List of References $\quad 134$

Appendix A Additional Accuracy Tests 140

A.1 Beacon Setup . . . . . . . . . . . . . . . 140

A.2 Propellers Running . . . . . . . . . . . . . . . . . . . . . . . . 141

A.3 Receiver Mounting Location on Quadrotor . . . . . . . . . . . . . . . 142

Appendix B Outdoor Flight Testing $\quad 145$

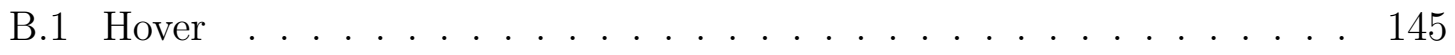

B.2 Landing . . . . . . . . . . . . . . . . . . . 147 
B.3 GPS ULS Combined Mission . . . . . . . . . . . . . . . 150

$\begin{array}{ll}\text { Appendix C Wake Velocity } & 154\end{array}$ 


\section{List of Tables}

2.1 GPS Accuracy (95\% Confidence) $[1] \ldots \ldots$. . . . . . . . . 8

2.2 Experimental Results Summary ('-' does not apply) . . . . . . . . . 33

3.1 GPS Accuracy . . . . . . . . . . . . . . . . . 42

3.2 Position Stabilization Gains . . . . . . . . . . . . . . 45

3.3 Summary of PID Gains . . . . . . . . . . . . . . . . . . . . 48

3.4 Attitude Stabilization Gains . . . . . . . . . . . . . . . . . . . 50

3.5 Altitude Stabilization Gains . . . . . . . . . . . . . . 51

4.1 Summary of the receiver positions . . . . . . . . . . . . 58

4.2 Band pass Filter Parameters . . . . . . . . . . . . . . . . 67

4.3 Summary of the analytical method rotation angles . . . . . . . . . 78

5.1 GPS Accuracy . . . . . . . . . . . . . . . . . . 123

C.1 Rotor velocity calculation . . . . . . . . . . . . . 155 


\section{List of Figures}

2.1 Vision-based state estimation control scheme . . . . . . . . . . . . . 10

2.2 Vision-based perception control scheme . . . . . . . . . . . . . . 10

2.3 Threshold detection . . . . . . . . . . . . . . . . . . . . . . 18

2.4 Envelope detection . . . . . . . . . . . . . . . . . . 20

2.5 3D Lateration Problem . . . . . . . . . . . . . . . . . . . . 21

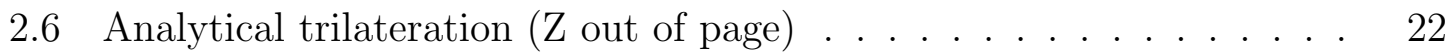

3.1 TARA 3 layout . . . . . . . . . . . . . . . . . 39

3.2 RPi position control block diagram . . . . . . . . . . . . . . . 44

3.3 Experimental flight data for finding $K_{u}$ and $T_{u} \ldots \ldots \ldots$. . . . 47

3.4 Implemented control scheme . . . . . . . . . . . . . . . . . . . . 49

3.5 Altitude hold control block diagram . . . . . . . . . . . . . . . . 52

4.1 Typical ultrasonic signal produced by the beacon . . . . . . . . . 55

4.2 Typical ultrasonic signal received by a receiver $1 \mathrm{~m}$ away . . . . . . . 56

4.3 Multilateration shown on quadrotor (X into the page) . . . . . . . 57

4.4 Receiver layout on quadrotor (Z into the page) . . . . . . . . . 57

4.5 Ultrasonic Localization System block diagram . . . . . . . . . . . . 59

4.6 Ultrasonic transducer schematic $[2] \ldots \ldots$. . . . . . . . . . . . . 61

4.7 Transmission length accuracy $\mathrm{P}(100,0,177) \ldots \ldots 2$

4.8 Beacon signal amplifier . . . . . . . . . . . . . . 63

4.9 Beacon top view . . . . . . . . . . . . . . . . 64 
4.10 Wire connections . . . . . . . . . . . . . . . 65

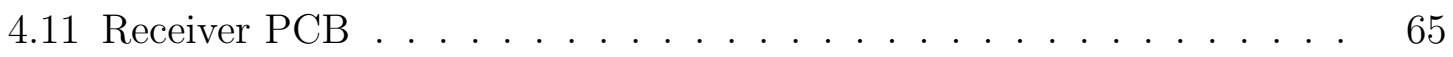

4.12 Receiver sensitivity $[2] \ldots \ldots \ldots \ldots \ldots$

$4.13 \mathrm{PSoC}$ ToF digital logic $\ldots \ldots \ldots \ldots$

4.14 Edge holder block . . . . . . . . . . . . . . . . . . 70

4.15 Time Stamp Controller block . . . . . . . . . . . . . . . 71

4.16 Stamp channel block . . . . . . . . . . . . . . . . . . . . 71

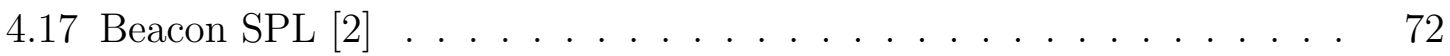

4.18 Receiver sensitivity $[2] \ldots \ldots \ldots \ldots \ldots$

4.19 Attenuation of the ultrasonic signal $[2] \ldots \ldots \ldots \ldots$

4.20 Beacon beam pattern $[2] \ldots \ldots \ldots \ldots \ldots$

4.21 Sensitivity beam pattern $[2] \ldots \ldots \ldots \ldots \ldots$

4.22 Analytical local coordinate frame top view . . . . . . . . 75

4.23 Analytical local coordinate frame side view . . . . . . . . 76

4.24 Low GDoP . . . . . . . . . . . . . . . . . 86

4.25 High GDoP $\ldots \ldots \ldots \ldots \ldots \ldots \ldots$

4.26 Dilution of precision at different locations . . . . . . . . . . . 92

4.27 PSoC analytical method block diagram . . . . . . . . . . . 98

4.28 PSoC block diagram LLS . . . . . . . . . . . . . . . . . . . . . 99

4.29 Transmission rate accuracy $\mathrm{P}(100,0,177) \ldots \ldots \ldots \ldots$

5.1 Accuracy testing . . . . . . . . . . . . . . . . . 102

5.2 Trilateration position accuracy . . . . . . . . . . . . . . . 104

5.3 Four-receiver LLS position accuracy . . . . . . . . . . . . . 106

5.4 Five-receiver LLS position accuracy . . . . . . . . . . . . . . 107

5.5 GPS-denied flight testing (X into the page) . . . . . . . . 108

5.6 Hovering in GPS-denied environment using three-receiver algorithm . 109

5.7 Hovering in GPS-denied environment using four-receiver algorithm . . 110 
5.8 Hovering in GPS-denied environment using five-receiver algorithm . . 111

5.9 PSoC block diagram combined lateration algorithm . . . . . . . . . . 113

5.10 Combined lateration position accuracy . . . . . . . . . . . . . 115

5.11 Hovering in GPS-denied environment using combined lateration algo-

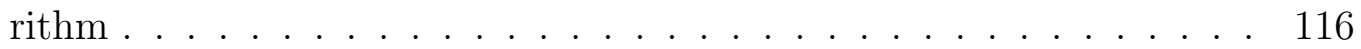

5.12 Extended hovering in GPS-denied environment using combined later-

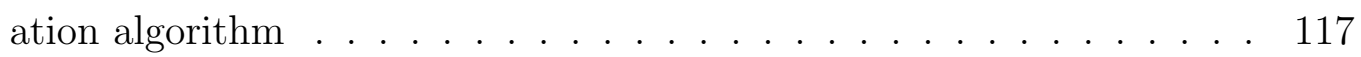

5.13 Extended hovering altitude using combined lateration algorithm . . . 118

5.14 Landing flow diagram . . . . . . . . . . . . . . . . . . . 119

5.15 Landing visualization . . . . . . . . . . . . . . . . 120

5.16 Landing in GPS-denied environment . . . . . . . . . . . . . . . 120

5.17 Outdoor flight-testing using TARA $3 \ldots \ldots$. . . . . . . . 122

5.18 ULS - GPS comparison, X axis . . . . . . . . . . . . 124

5.19 ULS - GPS comparison, Y axis . . . . . . . . . . . . . 124

5.20 ULS - GPS comparison, Z axis . . . . . . . . . . . . . . . 124

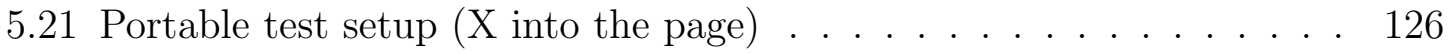

5.22 Portable beacon autonomous hover . . . . . . . . . . . . . . . . 127

5.23 Portable beacon autonomous landing . . . . . . . . . . . . . . . . . 129

A.1 Position accuracy of a single transducer . . . . . . . . . . . . . . . . . 141

A.2 Position accuracy of the full beacon . . . . . . . . . . . . . . 141

A.3 Position accuracy with propellers at half hover throttle . . . . . . . . 142

A.4 Position accuracy with no motors . . . . . . . . . . . . . . . . 142

A.5 Orthogonal receiver configuration . . . . . . . . . . . . 143

A.6 Position accuracy of receivers mounted on motor arms . . . . . . . . 144

A.7 Position accuracy of the orthogonal layout . . . . . . . . . . . . . . . 144

B.1 Hover in an outdoor environment using the ULS . . . . . . . . . . 147

B.2 Hover in an outdoor environment using GPS . . . . . . . . . . . . . 148 
B.3 Landing in an outdoor environment using the ULS . . . . . . . . . . 149

B.4 Landing in an outdoor environment using the GPS system . . . . . 150

B.5 Mission profile . . . . . . . . . . . . . . . . 151

B.6 Mission in an outdoor environment using the ULS . . . . . . . . . . . 152

B.7 Mission in an outdoor environment using the GPS . . . . . . . . . 153 


\section{List of Acronyms}

\begin{tabular}{|c|c|}
\hline Acronyms & Definition \\
\hline AoA & Angle of Arrival \\
\hline $\mathrm{BT}$ & Bluetooth \\
\hline BVLoS & Beyond Visual Line of Sight \\
\hline $\mathrm{CC}$ & Cross-Correlation \\
\hline CDMA & Code Division Multiple Access \\
\hline DGPS & Differential Global Positioning System \\
\hline DMA & Direct Memory Access \\
\hline DOP & Dilution of Precision \\
\hline EMI & Electromagnetic Interference \\
\hline $\mathrm{EKF}$ & Extended Kalman Filter \\
\hline FDMA & Frequency Division Multiple Access \\
\hline FHSS & Frequency Hop Spread Spectrum \\
\hline FIFO & First in First out \\
\hline
\end{tabular}




\begin{tabular}{|c|c|}
\hline FPS & Frames per Second \\
\hline GDoP & Geometric Dilution of Precision \\
\hline GNSS & Global Navigation Satellite System \\
\hline GPS & Global Positioning System \\
\hline HDoP & Horizontal Dilution of Precision \\
\hline IMU & Inertial Measurement Unit \\
\hline IR & Infrared \\
\hline $\mathrm{LS}$ & Least Squares \\
\hline LLS & Linear Least Squares \\
\hline LSB & Least Significant Byte \\
\hline MAV & Micro Aerial Vehicle \\
\hline MSB & Most Significant Byte \\
\hline NLS & Non-Linear Least Squares \\
\hline OGV & On Ground Vision \\
\hline $\mathrm{PCB}$ & Printed Circuit Board \\
\hline $\mathrm{PP}$ & Peak to Peak \\
\hline PoA & Phase of Arrival \\
\hline PID & Proportional Integral Derivative \\
\hline PSoC & Programmable System on Chip \\
\hline
\end{tabular}




$\begin{array}{ll}\text { PWM } & \text { Pulse Width Modulated } \\ \text { RF } & \text { Radio Frequency } \\ \text { RFID } & \text { Radio Frequency Identification Device } \\ \text { RMS } & \text { Root Mean Square } \\ \text { RMSE } & \text { Root Mean Square Error } \\ \text { RPi } & \text { Raspberry Pi } \\ \text { RSS } & \text { Received Signal Strength } \\ \text { RTK } & \text { Real Time Kinematic } \\ \text { SLAM } & \text { Simultaneous Localization and Mapping } \\ \text { RToF } & \text { Return Time of Flight } \\ \text { TDoA } & \text { Time Difference of Arrival } \\ \text { VDMA } & \text { Time Division Multiple Access } \\ \text { ULS } & \text { Visual Line of Sight } \\ \text { TGRN } & \text { Target Relative Navigation } \\ \text { UWB } & \text { Time of Flight } \\ \text { Ultra-wideband }\end{array}$


WAAS Wide Area Augmentation System

Z-N Ziegler-Nichols Tuning 


\section{List of Symbols}

\begin{tabular}{ll}
\hline Symbols & Definition \\
\hline \hline$A(t)$ & Matrix of Coefficient's for LLS \\
$a$ & Amplitude of Oscillation \\
$a_{x}$ & Speed of Sound \\
& Distance to Second Receiver in Local Coordinate Frame \\
$B$ & Centered on Receiver \\
$b$ & State Transition Matrix \\
$b_{x}, b_{y}$ & Matrix of Constants for LLS \\
& Distance to Third Receiver in Local Coordinate Frame \\
$C$ & Centered on Receiver \\
$D$ & Dilution of Precision Matrix \\
$d t$ & Distance Measurement Matrix \\
$E$ & Distance to Beacon \\
& Change in Time
\end{tabular}




\begin{tabular}{|c|c|}
\hline$e$ & Distance Error Matrix \\
\hline$f_{c}$ & Center Frequency \\
\hline$G_{k}$ & Process Noise Model \\
\hline$H_{k}$ & Observation Matrix \\
\hline$I$ & Sound Intensity \\
\hline$J$ & LLS Cost Function \\
\hline$K_{D}$ & Derivative Gain \\
\hline$K_{I}$ & Integral Gain \\
\hline$K_{P}$ & Proportional Gain \\
\hline$K_{u}$ & Ultimate Gain for Stability \\
\hline$m$ & Number of Subsets \\
\hline$n$ & Number of Receivers \\
\hline$P$ & $\begin{array}{l}\text { Position of the Beacon Relative to the Center of the } \\
\text { Quadrotor }\end{array}$ \\
\hline$P_{L}$ & Sound Power Level \\
\hline$p$ & Pressure \\
\hline$p_{0}$ & Reference Pressure \\
\hline$Q_{d}$ & Dilution of Precision Co-variance Matrix \\
\hline$Q_{k}$ & Process Co-variance Matrix \\
\hline
\end{tabular}




\begin{tabular}{|c|c|}
\hline$R$ & position of the receiver \\
\hline$R_{g}$ & specific gas constant \\
\hline$R_{x y}$ & Radius in XY plane \\
\hline$S$ & Receiver Sensitivity \\
\hline$S_{0}$ & Reference Sensitivity \\
\hline$S P L$ & Sound Pressure Level \\
\hline$T$ & Air Temperature \\
\hline$T_{u}$ & Period of Oscillation for Stability Limit \\
\hline$t$ & Time \\
\hline$U$ & Control Outputs \\
\hline$u$ & Measurement Noise \\
\hline V & Measurement Noise Matrix \\
\hline$W_{x}, W_{z}$ & Rotation Matrix \\
\hline$w$ & Process Noise \\
\hline$X$ & State Vector \\
\hline$x, y, z$ & Position \\
\hline$\dot{x}, \dot{y}, \dot{z}$ & Velocity \\
\hline$\ddot{x}, \ddot{y}, \ddot{z}$ & Acceleration \\
\hline$\alpha, \beta$ & Local Coordinate Frame Rotations \\
\hline
\end{tabular}


$\epsilon$

$\gamma$

$\gamma_{g}$

$\zeta_{k}$

$\lambda$

$\mu$

$\delta$

$\sigma$

$\phi, \Theta, \psi$

$\dot{\phi}, \dot{\Theta}, \dot{\psi}$

Superscript

I

\section{Subscript}

$d$

E

G

$k$

$E$
Sonar noise

Quadrotor Position Angle

Heat Capacity Ratio

Measurement Vector

Wavelength

Unit Vector

Phase

Variance

Roll, Pitch and Yaw Angles

Roll, Pitch and Yaw Rates
Local Coordinate Frame Centered on Receiver

True Measurement

Mean Measurement
$d$
Desired

Pixhawk Extended Kalman Filtered States

G Raw GPS

$k \quad$ Kalman filtered states 
$p$

$r$

$s$
Position of the Beacon

Position of the Receiver

Sonar

xxiv 


\section{Chapter 1}

\section{Introduction}

\subsection{Overview and Motivation}

Quadrotors have become increasingly popular over the years, where they are involved in many jobs and hobbies. Typical unmanned aerial vehicle (UAV) missions can be referred to as the 5 Ds. These represent dull, dirty, dangerous, denied, and dash. Dull missions include a persistent surveillance or extended missions. Dirty represents pollution or agricultural monitoring. Dangerous in monitoring wind turbines or hostile environments. Denied such as operating indoors and dash such as rapid delivery [3] [4]. To complete these missions a quadrotor must know where it is for obstacle avoidance and path planning and requires a method for localization.

Localization is the process of determining the location of objects or people. As humans, we use our senses to locate objects and people relative to ourselves. Our brain uses all our senses to give us a picture of the world around us. Quadrotors require sensors to see the world. They also combine different measurements to obtain a more accurate picture.

The Global Navigation Satellite System (GNSS) is one of the most widely used systems for localization. This includes the Global Positioning System (GPS), GLONASS, and Galileo systems. For some applications the standard GPS accuracy is insufficient 
such as for a precision landing. Other applications require operation in a GPS denied environment. In hostile environments, quadrotors are useful for reconnaissance. They provide information about the surroundings through video or thermal imaging [5]. A GPS signal can be tracked or spoofed. The signal can be tracked when the GPS coordinate is transmitted to a base station. In other environments GPS may be unavailable due to interference, such as for indoor localization or under a forest canopy. In addition, relying solely on GPS for localization presents a vulnerability and additional options need to be explored.

Google's Wing aviation and Drone Delivery Canada are currently the leaders for drone delivery [6]. They can achieve this by working closer with the regulators. Google's wing aviation has recently been certified by the Federal Aviation Administration (FAA) to perform drone delivery in Virginia [7]. Drone Delivery Canada has been working with Transport Canada on Beyond Visual Line of Sight (BVLoS) trials to help create regulations [8]. Current Drone Delivery Canada trials are performed using radar to augment the GPS capabilities. One of the largest aspects impacting regulations on urban flying is a localization system if GPS signal is lost [9]. Large scale localization systems in urban centers could augment GPS capabilities. This would mitigate some issues for drone delivery in urban centers.

Small scale localization can provide increased accuracy for the critical phases of flight. The drones can fly at designated flight levels for cruise and have access to a clear sky for GPS. The landing phase of any delivery is the most dangerous, due to obstacles and a possible loss of GPS signal. Another localization method is the most important during this phase of flight. When a user is expecting a package, they can place a landing pad or beacon for close range localization. This allows for increased accuracy and reliability for landing. 


\subsection{Problem Statement}

The goal of this thesis is to perform ultrasonic localization of a quadrotor using a portable beacon and to integrate the control system with commercially available hardware in a GPS denied environment.

Ultrasound offers a relatively simple and cheap solution over alternative hardware. Low cost hardware allows for a commercially viable product. The main limitation of ultrasound is the range due to the mechanical nature of the wave. The principles can be expanded to other hardware in the future.

The localization system should interface with commercially available hardware. The system should provide outputs like GPS which can be used in a larger system. Integrating the system into a commercially available product shows the feasibility for implementation. For this application a Pixhawk autopilot is selected.

A portable beacon allows for a more versatile system. Typical ultrasonic localization systems, nodes are placed at known and fixed locations. This requires precise setup and limits localization to this location. Mounting the nodes on the quadrotor allows for a portable beacon. This allows for an easy implementation of the system in different locations. It allows for the capability of implementing it on a moving object or person.

\subsection{Organization of Thesis}

In chapter 2 , the results of a literature review are discussed. First an overview of the technologies is discussed. Based on related work, localization using ultrasound typically uses lateration. A lateration problem can be broken down into three steps. This begins with ranging techniques, then detection methods and finally lateration techniques. 
The flight platform and the required software for flight are discussed in Chapter 3. It provides an overview of the sensors required for quadrotor flight using a Pixhawk as the main hardware component. Next the software is introduced and the control schemes for the different flights that are tested.

The ultrasonic localization system (ULS) is discussed in chapter 4. An introduction into time of flight localization is provided which is required to understand the following hardware choices. The hardware is introduced with the main purpose of determining the time of flight of an ultrasonic signal. Next the software is covered which is required to determine the position of the quadrotor.

In chapter 5, first the performance and accuracy of the ULS is discussed using different lateration algorithms. Flight data for hover and landing in a GPS denied environment is analyzed. A new lateration algorithm is developed to improve the position accuracy and update rate. The ULS performance is compared to GPS performance. Finally the performance using a moving beacon is tested in a GPS denied environment through hover and landing.

The conclusion to the research and future possibilities are provided in chapter 6 . 


\section{Chapter 2}

\section{Literature Review}

\subsection{Overview}

There are a variety of different methods for localization with a tradeoff on range, complexity, and accuracy. One method for localization is lateration. In lateration the first step is to determine the distances between the transmitter and the receivers. The next step is to determine if a signal is received which presents a tradeoff between complexity and noise rejection to determine the true distance. These ranges are then combined to produce an estimate of the position using one of the lateration techniques. The lateration problem can be broken down into four main steps: the localization method, ranging technique, detection method and lateration technique.

The lateration related experiments are introduced once the localization and lateration concepts have been covered. The experiments provide insight into the performed localization configurations and the corresponding accuracies. 


\subsection{Localization Methods}

\subsubsection{Global Navigation Satellite System}

The GNSS is one of the most widely used systems for localization. There are four main systems which include Global Positioning System (GPS), GLONASS, Galileo, and Beidou.

GPS is the most widely used satellite system and is owned by the American military. It consists of up to 32 satellites on 6 different medium earth orbits. GLONASS is operated by the Russian defense forces and consists of up to 24 satellites. Galileo will be operated by the European Space Agency and is estimated to be fully functional by 2020. BeiDou is a Chinese operated satellite system which currently only covers the Asia-Pacific area with 16 satellites and intends to reach global coverage by 2020 with 35 satellites [10].

Localization for the satellite system is a lateration problem which uses a ToF method. The position of each satellite is configured when the GPS is first powered on. Each satellite transmits a pseudo-random code to the receiver which the receiver can decipher to determine when the message is sent. The time on each satellite is based on an atomic clock accurate up to $10 \mathrm{~ns}$ [11]. The signal travels at the speed of the light and can be used to determine the distance to each satellite. The receivers use a quartz clock which is less accurate than an atomic clock. To correct for this inaccuracy the time offset is represented by a variable. Typically, linear least squares is used to solve the system of equations for the unknowns $(\mathrm{x}, \mathrm{y}, \mathrm{z}, \mathrm{t})$.

GPS accuracy with a $95 \%$ confidence level conducted by an independent study for the FAA is shown in Table 2.1 [1]. The vertical position error is larger than the horizontal position error due to the difference in horizontal dilution of precision (HDoP) and vertical dilution of precision (VDoP). Dilution of precision is discussed later in section 4.3.3. The user range error refers to the error in distance from the 
satellite to the user. The user range rate error gives insight to the error on the instantaneous position or relative position. The accuracy of the system is limited by atmospheric and local effects. Some of the effects are [12]:

1. Ionosphere: Slows the propagation speed due to an ionized atmosphere. The effect increases as the satellite moves towards the horizon and is frequency dependent. The error is mitigated by passing multiple frequencies and calculating the error for each. Military applications and expensive receivers have access to a second frequency whereas civilian applications have access to only one frequency.

2. Troposphere: The troposphere contains water molecules which effect the propagation speed. The effects are variable and change faster than the ionosphere.

3. Multipath Propagation: If there are nearby buildings or mountains, the signal can bounce off the surface and provide an inaccurate distance measurement.

4. Satellite Geometry: The positions of the satellites relative to each other can affect the multilateration calculation, this is referred to as Geometric Dilution of Precision (GDoP) and is discussed in further detail later.

5. Orbit: The receiver estimates the position and velocity of the satellites based on known orbits, however the orbits and velocity are not constant.

Differential GPS (DGPS) solves the position error down to within a few centimeters. DGPS is initially used for coastal applications to allow boats to navigate through busy harbors [13]. A fixed localized base station receives the pseudo-random code from each satellite, also known as local area augmentation system (LAAS). It calculates the error for each satellite and broadcasts it out to all nearby receivers. The difficulty with DGPS is the limitation in range to ensure the base station and 
Table 2.1: GPS Accuracy (95\% Confidence) [1]

\begin{tabular}{|c|c|c|}
\hline Parameter & Value & Unit \\
\hline \hline Horizontal Position Error & 1.891 & $\mathrm{~m}$ \\
\hline Vertical Position Error & 3.872 & $\mathrm{~m}$ \\
\hline User Range Error & 2.850 & $\mathrm{~m}$ \\
\hline Maximum User Range Rate Error & 0.05 & $\mathrm{~m} / \mathrm{s}$ \\
\hline
\end{tabular}

the receivers are experiencing the same propagation losses. This has been used for accurate positioning for quadrotors to enhance the accuracy over typical GPS [14] [15].

The FAA developed the Wide area augmentation system (WAAS) to provide accurate position measurements at airports. Like DGPS, ground stations measure the GPS satellite orbit inaccuracies, and delays caused by atmospheric and ionospheric attenuation. Correction factors are sent to geostationary satellites over the US which broadcast the errors to aircraft, using this method the error is $0.34 \mathrm{~m}$ with $95 \%$ confidence [16].

Real time kinematic (RTK) operates on the same principle as DGPS by sending a correction from a base station. However, RTK does not use the pseudo random code-based approach to determine the ToF of the signal. It uses a carrier-based approach, counting the number of carrier cycles between the receiver and satellite and multiplying by the wavelength to determine a distance. The base station sends out a phase difference correction factor allowing for sub wavelength accuracy. RTK has had reported accuracies of $6 \mathrm{~cm}$ [17]. 
Tansuriyong [18] used RTK to localize a drone for autonomous landing at a station to recharge. For accuracy testing, Tansuriyong walked around a rectangular course with a standard GPS and an RTK enhanced GPS several times. For the standard GPS there is significant scatter and does not resemble the course. For the RTK GPS there is an average $75 \mathrm{~cm}$ variation direction. For a landing test the drone landed at $50 \mathrm{~cm}$ away from the landing pad.

In many scenarios localization is required in GPS-denied environments due to interference or multi-path propagation such as in an indoor environment, near urban canyons or under dense forest coverage. This can also be the case while operating in hostile environments where the GPS signal can be tracked so a local localization method is required [19]. The GPS signal can be tracked if the quadrotor is transmitting the GPS location to a base station. GPS spoofing can also be an issue while operating near hostiles. A spoofer sends code-based signals like GPS and the receiver reads this as the pseudo random code. The spoofer can inject these fake GPS signals to take control of the autonomous aircraft and conduct a mission or crash. The spoofer is considered in control of the aircraft once it can provide the $3 \mathrm{D}$ position and velocity. Civilian GPS are much more prone to spoofing because there is less encryption and the pseudo random code is easier to predict.

\subsubsection{Vision-Based Localization}

Vision-based localization does not use lateration but is covered for completeness. There are two different approaches to vision-based localization systems, through state estimation and perception. State estimation is the process of determining an aircraft's $3 \mathrm{D}$ state. The vision system is part of the measurement block and would be used for feedback control which is shaded in Fig. 2.1. It provides inputs to the measurement block which can be used to replace a GPS. Perception uses the vision-based estimates to generate position and velocity inputs to the guidance system as a desired position 
or velocity set point as shown in red in Fig. 2.2. The controller uses GPS or another localization method to estimate its current position and velocity for feedback control. Since perception relies on another localization method for control it will not be discussed. There are two main state-estimation approaches that are discussed, on-ground vision, and target relative navigation. Kendoul [20] has a more complete review of vision-based approaches.

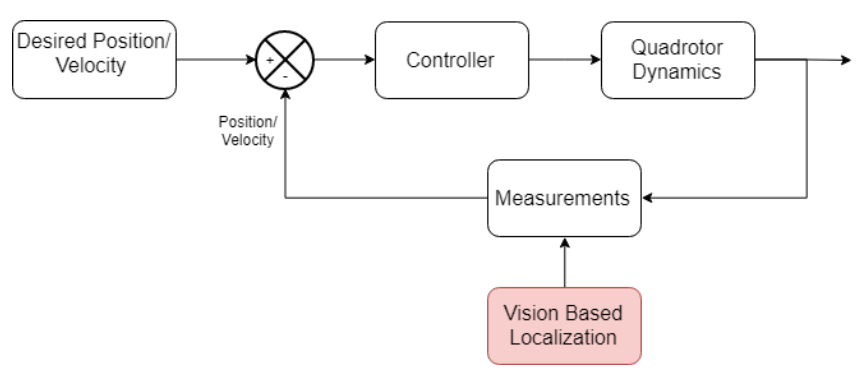

Figure 2.1: Vision-based state estimation control scheme

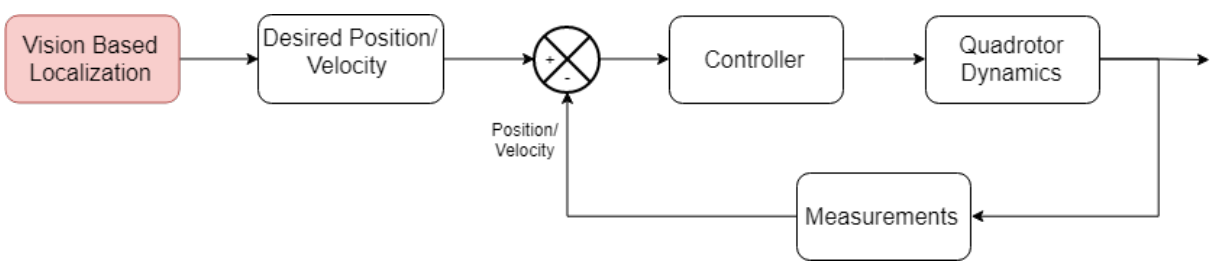

Figure 2.2: Vision-based perception control scheme

On-ground vision consists of camera's being placed on the ground to track an aircraft state. This reduces payload but camera infrastructure must be in place. This limits the application to landing and near field object tracking. Typically, the cameras track coloured landmarks on the object. Multiple cameras can be oriented to perform multilateration. An example of on-ground vision is work done at the Universidad Politecnica de Madrid [21] where researchers are able to achieve autonomous landing and hovering. For in-flight accuracies the university compares position estimates from their vision system with positions from a standard GPS fused with an inertial measurement unit (IMU) with a lateral root mean square error (RMSE) of $75.5 \mathrm{~cm}$. 
There is no stationary accuracy data and it is tough to gauge the accuracy of the system since both measurements have error.

Target relative navigation is the process of detecting a specific target to estimate position. This approach requires a visual target which is always within the field of view of the camera. There are two main applications which this is used for, visionbased landing on a known target and vision-based mobile target tracking [20]. The landing target is designed to simplify image processing and to allow the orientation to be determined from all angles. The pattern and size of the landing pad is known, allowing an aircraft to determine the scale factor and determine its relative position and velocity to the landing pad.

Some examples of target relative navigation using a known target include, Lange [22] uses a black and white circular pattern to determine the center cut out in a hexagonal shape to determine orientation. The main drawback of this design is that the entire pattern needs to be in sight at all times. Stationary position tests have an maximum error of less than $5 \mathrm{~cm}$. Tests of his system showed a horizontal standard deviation of $3.8 \mathrm{~cm}$ while hovering over a landing pad for five minutes using an off board camera to determine the ground truth position. There is not any accuracy data related to landing. Bosnak [23] used a unique landing pad to determine the aircraft state relative to this landing pad and is able to successfully perform directed positioning over the landing pad as well as landing. This system uses off-board processing which results in a low frequency update and thus delays which are compensated for using Kalman filtering techniques. In flight hover data is presented but is not compared against truth data for accuracy assessment. Cocchioni [24] expands on a single landing pad by using four unique designs, one for each leg of the quadrotor. Each leg fits into a copper fitting to charge the quadrotor when on the landing pad. This allows the quadrotor to autonomously land and take off when fully charged. A lateral static position accuracy of less than $2 \mathrm{~cm}$ is obtained at a height less than $2 \mathrm{~cm}$. In 
flight accuracy is not assessed against truth data.

Vision-based mobile target tracking creates difficulties because the relative velocity is determined rather than the absolute velocity. The algorithm for image processing must be able to track the specific target as the object moves. Examples using an unknown target include, Lee [25] who is able to perform this using a Pixhawk. Due to the processing requirements the object is limited to move one metre or less and has to wait for the quadrotor to reposition. The main limitations are the low performance processing board and the image recognition delays using OpenCV. There are no accuracies reported with this system. Another system designed by Georgia Tech [26] is used to track a white van by estimating the contour of the van. Center only relative state estimation tracked only the center of the van and uses an extended Kalman filter to estimate the states. A second approach named subtended angle relative state estimation tracks both the center of the contour as well as the corners. Both approaches can successfully track the van, but the scale factor is unknown since the size of the van is unknown which leads to inaccurate distances to the van. There are not any reported accuracies related with this system. Wang [27] successfully lands a quadrotor using vision-based methods on a vessel deck by incorporating the motions of the vessel. In flight hover accuracy is assessed with a stationary platform and a moving platform using a Vicon motion capture system to assess the accuracy. The average error is less than $2 \mathrm{~cm}$ for a stationary platform and less than $3 \mathrm{~cm}$ for a moving platform at an altitude of $100 \mathrm{~cm}$. For stationary landing the average distance error from the desired landing position is $7.9 \mathrm{~cm}$ and it is $12 \mathrm{~cm}$ for a moving landing pad.

\subsubsection{Ultrasound Localization}

Ultrasound (US) consists of pressure waves oscillating at a frequencies greater than 20 $\mathrm{kHz}$. It has a relatively low detection range reaching up to a maximum of $30 \mathrm{~m}$ [28]. 
As the signal propagates at the speed of sound, this allows for decreased complexity in processing hardware. Experimental data is provided after introducing lateration techniques.

\subsubsection{Ultra-wideband Localization}

Ultra-wideband (UWB) spreads information over a wide range of the frequency spectrum allowing a large amount of data to be transferred with low energy consumption. It uses short pulses, less than $1 \mathrm{~ns}$, over a bandwidth greater than $500 \mathrm{MHz}$ [29]. It does not interfere with other frequencies within the spectrum because the individual frequency amplitude is low. UWB can penetrate walls effectively because it occupies a large portion of the frequency spectrum. Current Canadian laws regulate the UWB emissions per frequency band and have more strict regulations for outdoor use than indoor use per RS-220 [30]. Experimental data is provided after introducing lateration techniques.

\subsubsection{Infrared Localization}

Infrared (IR) operates using invisible light just below visible red. Typical localization setups use diffuse IR, these setups emit IR in all directions. Receivers can be positioned within rooms to detect if a transmitter is nearby or measure the incoming angle for triangulation. Experimental data is provided after introducing lateration techniques.

\subsubsection{WiFi Localization}

WiFi, otherwise known as the IEEE 802.11 standard, is popular to provide internet access in private, commercial, or public places. WiFi typically has a range of 100 $\mathrm{m}[29]$ in an open environment but interference and attenuation reduce this range. 
Typical accuracy of systems ranges between $1 \mathrm{~m}$ to $30 \mathrm{~m}$ [31]. Experimental data is provided after introducing lateration techniques.

\subsubsection{Bluetooth Localization}

Bluetooth (BT), or IEEE 802.15.1, operates on the $2.4 \mathrm{GHz}$ frequency band and it is designed for low power peer-peer communication. The range of Bluetooth is approximately $10 \mathrm{~cm}$ to $10 \mathrm{~m}$. Due to the short communication range and fast propagation speed it is mostly limited to received signal strength localization [31]. Experimental data is provided after introducing lateration techniques.

\subsubsection{Radio Frequency Identification Device Localization}

A Radio Frequency Identification Device (RFID) system consists of tags which emit data and readers to receive the data. Each tag and reader have a specific frequency and protocol which are used to communicate [29]. There are two main types of RFID systems active and passive. Active systems have a power source and can operate over

a range of hundreds of meters. Passive systems do not have a power source and are limited to a range of a few meters. Experimental data is provided after introducing lateration techniques.

\subsection{Ultrasonic Ranging Techniques}

The localization method used in this work is ultrasound because it is simple, cheap and can reach sub centimeter accuracy [32]. It provides a good basis to perform GPS-denied localization which can be expanded to other localization methods in the future. Therefore, ranging techniques compatible with ultrasonic localization will be discussed. 
Every lateration problem requires distance measurements to the target from three or more sources. The ranging techniques section introduces different methods which these distances can be obtained using ultrasound.

\subsubsection{Received Signal Strength}

Received Signal Strength (RSS) is the easiest solution to implement for localization. RSS is based on the fact that the the received power decreases as the distance between the transmitter and receiver increases. If the transmitting power is known, the distance can be determined using a signal propagation model. For ultrasound the signal propagation model follows the inverse square law of the distance from the transmitter to the receiver, given by Eq. 2.1 (where $I$ is the measured intensity, $P W L$ is the transmitted sound power level, and $d$ is the calculated distance). The distance represents the radius of a spherical surface of where the transmitter could lie centered on the receiver. RSS localization suffers from inaccuracies due to attenuation losses which include environmental and directional effects.

$$
I=\frac{P W L}{4 \pi d^{2}}
$$

\subsubsection{Time of Flight}

Time of flight (ToF) refers to the time the signal takes to travel from a transmitter to a receiver. It can be taken as the difference between the time of reception $\left(t_{R_{i}}\right.$ and time of transmission $\left(t_{T}\right)$ which requires the clocks on the beacon and receivers to be synchronized. This difference can be converted to a distance if the signal propagation speed is known as per Eq. 2.2. Similar to RSS, the distance represents a spherical surface. 


$$
d_{i}=a *\left(t_{R_{i}}-t_{T}\right)
$$

\subsubsection{Time Difference of Arrival}

Time difference of arrival (TDoA) follows the same principles as ToF but the time of the transmission is not required. For TDoA the ToF from one receiver is subtracted from the other receivers, thus eliminating the time of transmission from the calculation as shown by Eqs. 2.3 and 2.4. This reduces complexity as the clocks on the receiver and the transmitter need not need be synchronized. The transmitter is no longer located on the surface of a spherical surfaces centered on the receiver but rather on a hyperbolic surface. To determine a $3 \mathrm{D}$ position a minimum of four receivers are required as one receiver is taken as a reference.

$$
\begin{gathered}
d_{1}-d_{2}=a *\left(t_{R_{1}}-t_{T}\right)-a *\left(t_{R_{2}}-t_{T}\right) \\
d_{1}-d_{2}=a *\left(t_{R_{1}}-t_{R_{2}}\right)
\end{gathered}
$$

\subsubsection{Return Time of Flight}

Return time of flight (RToF) follows the same principle as ToF but once a signal is received a signal is transmitted back and received by the original transmitter. This technique also avoids clock synchronization between the transmitter and the receiver. It also mitigates one directional noise such as wind because the interference on the first transmission will be balanced by the second transmission. A major drawback is that a transmitter and a receiver are required at both locations. In addition, the localization update rate is decreased because the signal must propagate twice as far. 


\subsubsection{Phase of Arrival}

Phase of arrival (PoA) measures the phase shift of a sinusoidal wave between the transmitter and the receiver. The distance can be calculated by multiplying the wavelength by the difference of the receiver phase and the transmitter phase which requires clock synchronization. This requires the receivers to be within one wavelength of the transmitter which for a $40 \mathrm{kHz}$ ultrasonic wave is $8.5 \mathrm{~mm}$. Typically the receivers on the flying vehicle are outside of this range making this approach difficult. However, this approach can be used in combination with ToF or TDoA to provide sub wavelength accuracy.

\subsection{Ultrasonic ToF Detection Methods}

ToF is selected as the ranging technique in this thesis for several reasons. It provides more accurate results than received signal strength [31]. It requires one less receiver than TDoA and it is less complex than RToF with multiple receivers. Enhancing the accuracy using phase of arrival is not necessary as an accuracy less than $8.5 \mathrm{~mm}$ is not required and most likely unacheivable with timing errors. Clock synchronization between the beacon and receivers is performed using a radio frequency signal propagating at the speed of light.

To determine the ToF the transmission time and the reception time are required. The transmission time normally corresponds to when the signal is produced. There are different methods to determine the time of reception, ideally it is consistent at different ranges and different noise levels. A constant ramp up time can be subtracted from where the signal is detected to determine the true range. This is typically factored into an overall delay. 


\subsubsection{Threshold}

ToF detection methods refer to how the signal is captured by the receiver. Threshold detection is the fastest and easiest technique as once a signal is received with a certain power level the time stamp is captured. An example of threshold detection using a $40 \mathrm{kHz}$ ultrasonic wave is shown in Fig. 2.3. Before the third peak the signal passes the threshold, and this triggers that a signal is received. A constant ramp up time to reach this threshold can be subtracted to obtain the ToF of the signal. Threshold detection can lead to inconsistencies because it can be detected on different peaks depending on noise and range. This would make the ramp up time inconsistent and effects the accuracy of the ToF. This method can lead to range and noise issues. If the threshold value is set too high the detection range is limited because of signal attenuation. If the threshold is set too low the receiver is subject to noise and false triggering.

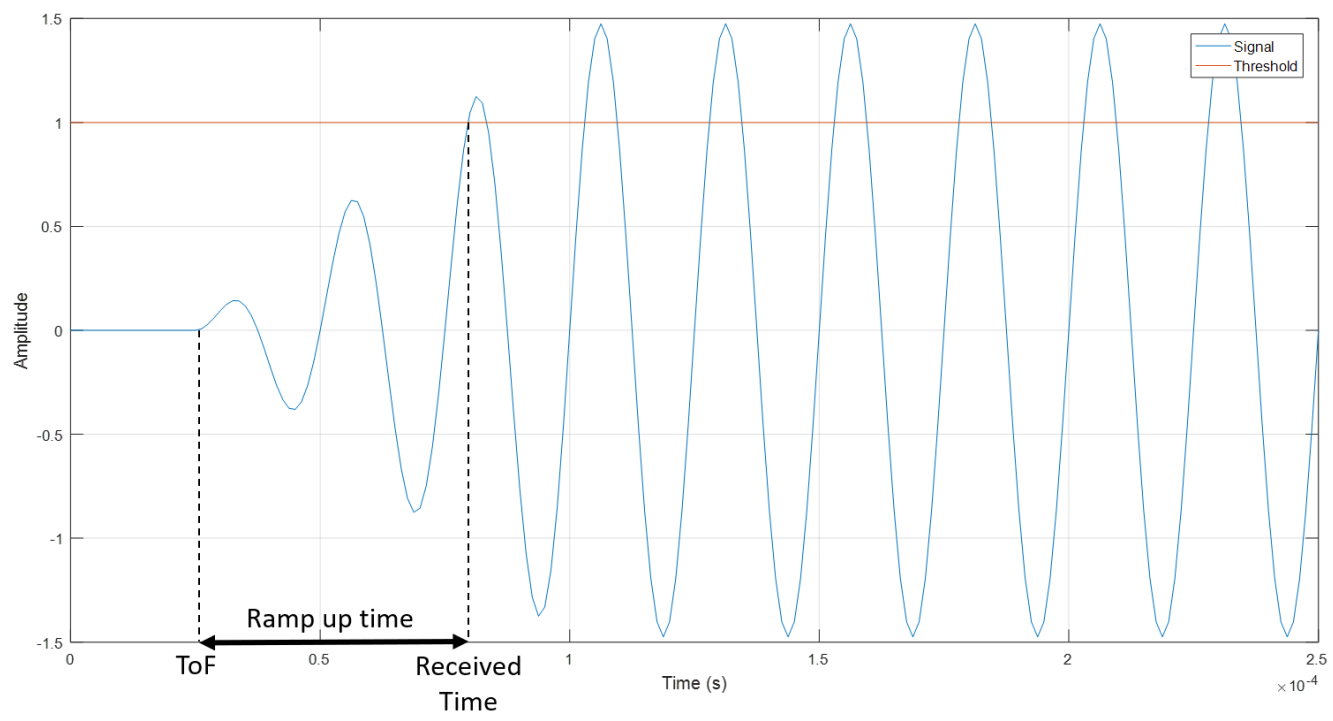

Figure 2.3: Threshold detection 


\subsubsection{Correlation Techniques}

Correlation techniques involve performing cross correlation (CC) with a measured signal and a reference signal. The reference signal is produced by the transmitter and is designed for the application by changing the amplitude or frequency as a function of time. Similar to threshold detection the signal can be requested and transmitted, or it can also be in constant transmission. If the signal is constantly transmitting the reference signal must be designed long enough such that the maximum detection range is outside of one wavelength. Amplitude modulation suffers decreased detection range when the amplitude of the transmitted wave is low. The received time can be determined when the correlation coefficient reaches a peak, this is when the transmitted signal and the reference signal are in phase. Some algorithms use the max peak, while others search for an earlier peak [32]. The ToF can be determined by subtracting the ramp up time. This method has increased range than the threshold technique because it does not require a signal threshold to filter out the noise. The noise is filtered out through the cross-correlation process since the correlation coefficient between white Gaussian noise and the reference signal will be zero.

Cross correlation allows for unique reference signals which can uniquely identify transmitters. These transmitters can be located at fixed locations and the unique identifier can be used for localization in large scale operations. Code division multiple access (CDMA) assigns each transmitter a unique code such as a sine wave with a specific frequency fluctuation, each code is set such that it is orthogonal to the other

local codes. In frequency hop spread spectrum (FHSS) a pseudo-random frequency hopping pattern is set for each transmitter. For both methods cross correlation is used to determine the time of flight of each transmitter. 


\subsubsection{Envelope}

Envelope detection develops an envelope around the signal and the peak of the envelope is used as the ToF shown in Fig. 2.4 for a $40 \mathrm{kHz}$ ultrasonic wave. The time to reach the maximum of the first peak of the envelope will be used as the received time and the ramp up time can be subtracted to obtain the ToF. The envelope has a more consistent detection than the threshold because the maximum of the envelope should be consistent depending on the range and noise. The Hilbert transform can be applied to determine the envelope, however, this is impractical for real time sampling.

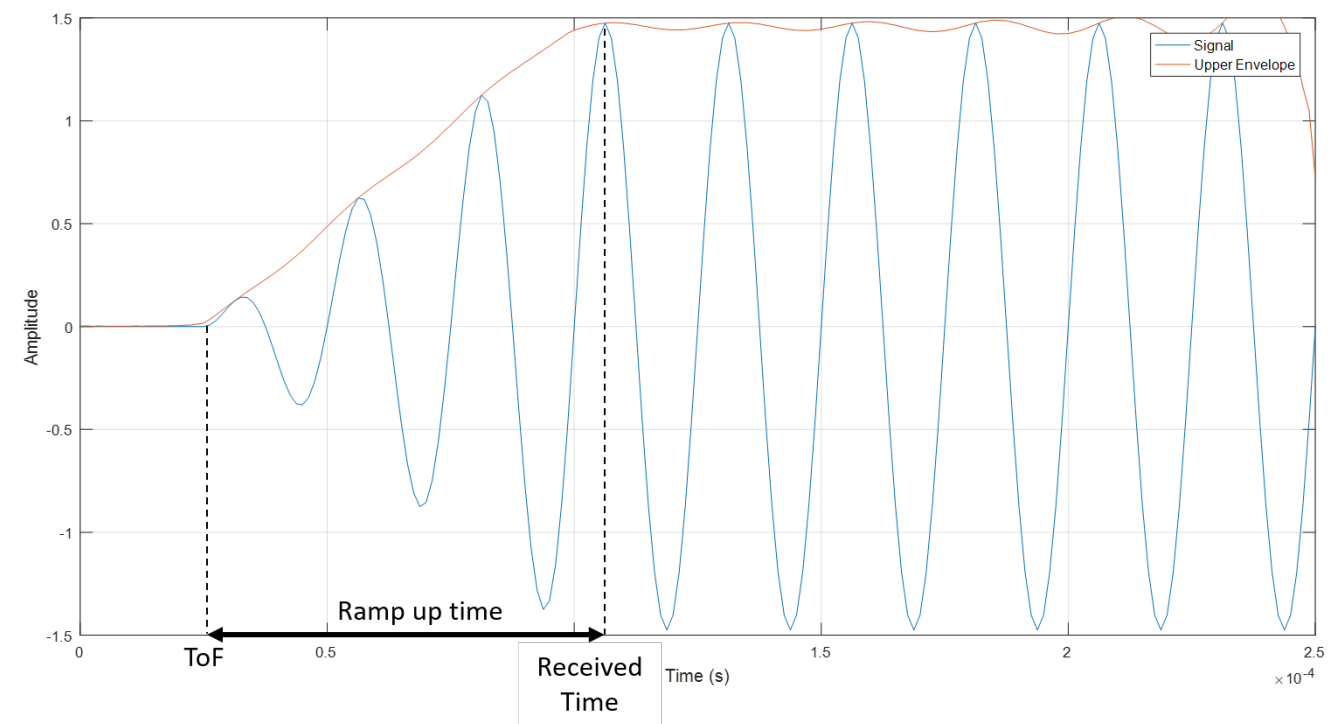

Figure 2.4: Envelope detection

\subsection{Ultrasonic ToF Lateration Techniques}

The selected detection method for this theis is threshold because it is fast and easy to implement. Cross correlation and envelope detection are computationally slower which limit the update rate for real time localization of a quadrotor.

Lateration techniques refer to the process of combining three or more distance 
measurements to obtain an estimate of position. Each distance measurement represents a spherical surface and at the intersection of three or more of these surfaces lies the position of the beacon, $P\left(x_{p}, y_{p}, z_{p}\right)$, as shown in Fig. 2.5. Most of these techniques assume that there are errors in each distance measurement and try to combine information in a manner to find the most accurate position.

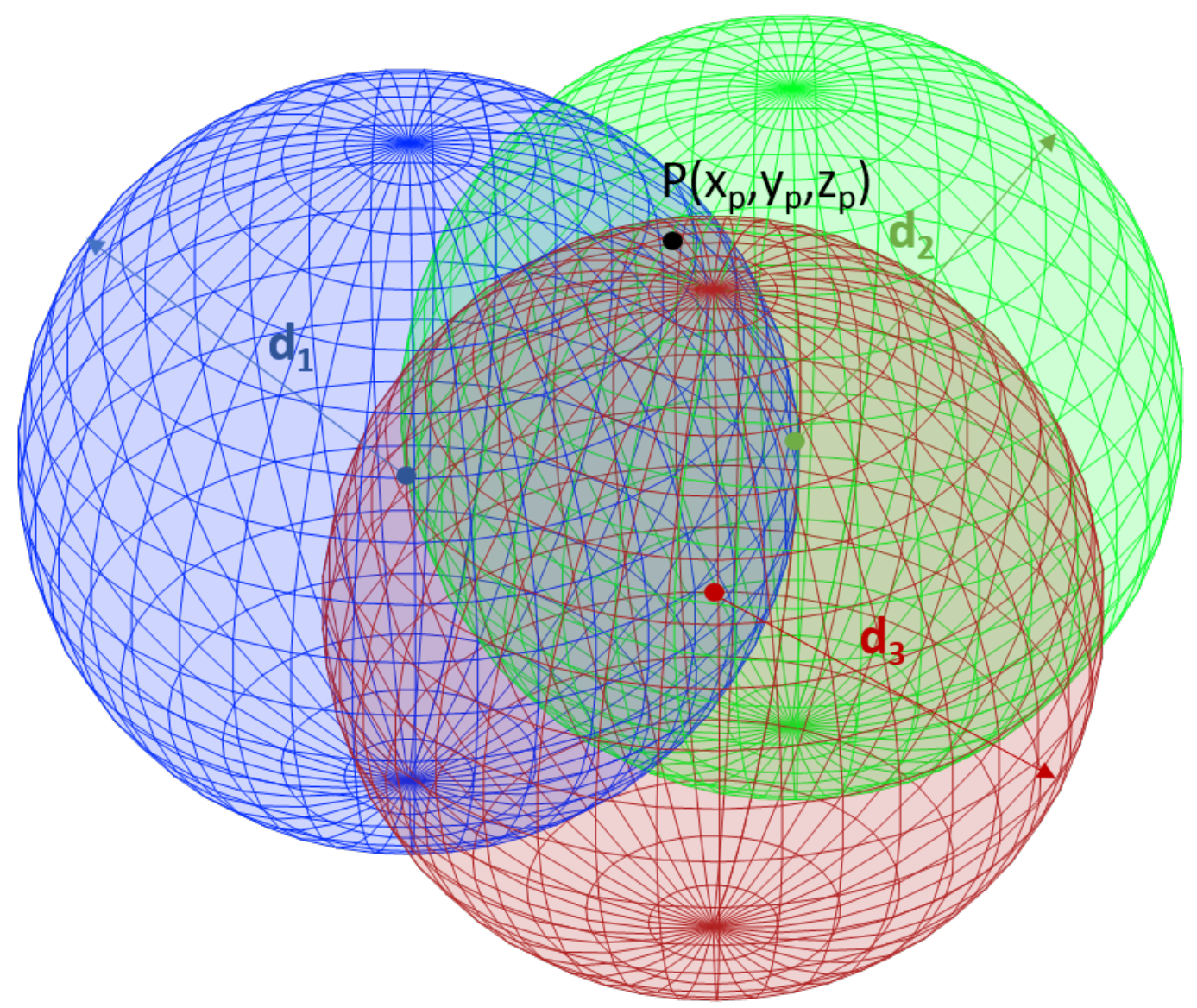

Figure 2.5: 3D Lateration Problem

\subsubsection{Analytical Trilateration}

A solution to the problem can be written if the coordinate frame of the receivers is selected according to Fig. 2.6. Any three independent receivers form a plane. The 
origin of this plane is selected to be at the first receiver, the second receiver is located along the $\mathrm{x}$ axis and the third receiver is located somewhere in the XY plane. A local coordinate frame (centered on the first receiver) can be used for this calculation and a transformation can be applied to change the calculated position into a local frame centered on the quadrotor. The geometry of the receivers is known which allows the transformation to be performed. An issue with this approach is that it does not consider that each distance measurement has errors and any error will have a direct impact on the position calculation.

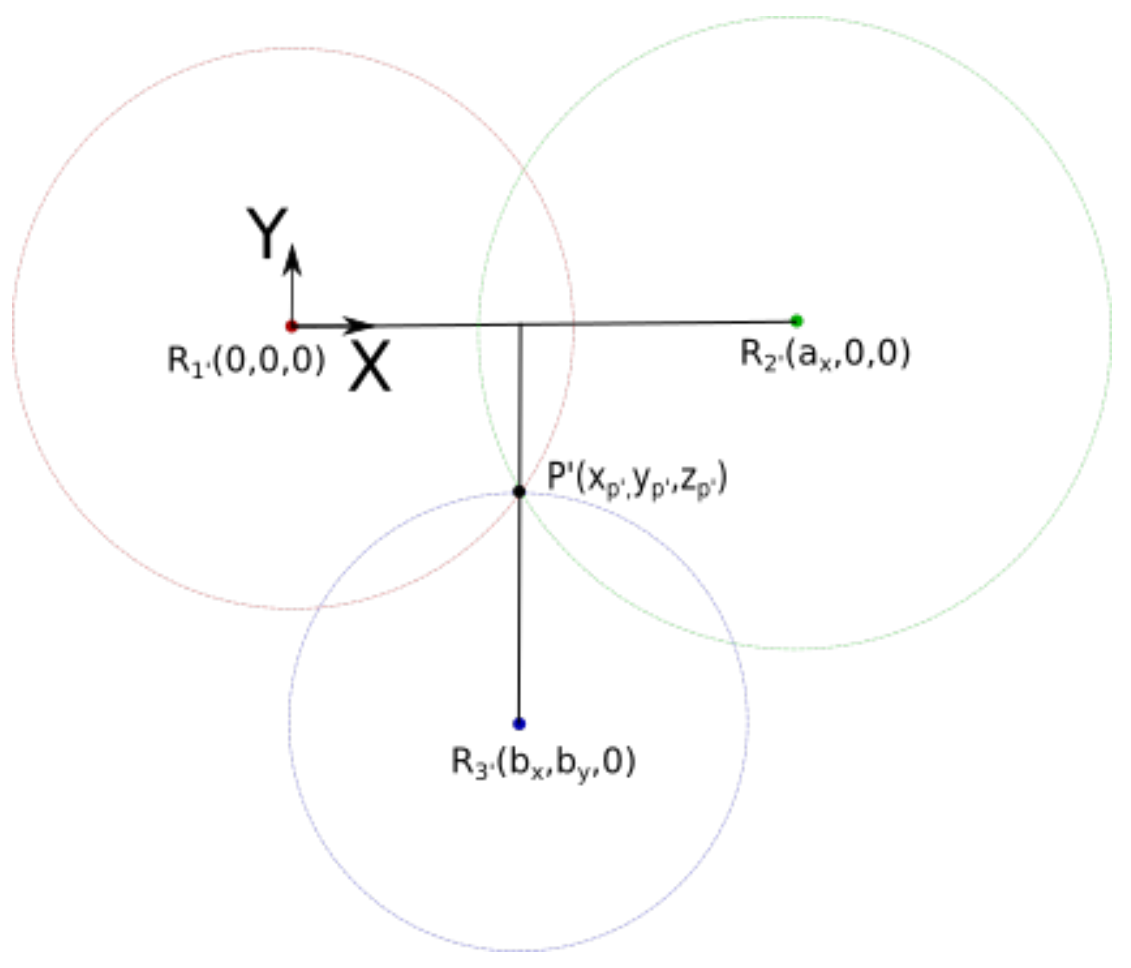

Figure 2.6: Analytical trilateration (Z out of page)

\subsubsection{Linear Least Squares}

Linear least squares (LLS) assumes each distance measurement has Gaussian error and solves for the solution that minimizes the error squared. LLS requires the original 
equation to be linear, this is done by subtracting each equation by a reference equation. A minimum of four receivers is required to obtain an LLS solution, using more receivers can decrease position error. Since LLS assumes the noise to be Gaussian it does not handle outliers well.

\subsubsection{Nonlinear Least Squares}

The nonlinear least squares (NLS) solution minimizes the error squared for a nonlinear function. The LLS solution provides the first estimate for the nonlinear solution. Using the first estimate the nonlinear set of equations are solved iteratively to find the NLS solution. This results in a more accurate solution than LLS for non-linear functions.

\subsubsection{Extended Kalman Filter}

A Kalman filter observes measurements over time to produce an estimate of the position. The EKF expands on the Kalman filter by expanding the model and observation equations for non-linear dynamics. The extended Kalman filter (EKF) approach assumes that both the process and measurement models can be modelled with Gaussian noise [33]. The 3D position and velocity represent the states of the filter.

\subsubsection{Subset Generation}

Subset generation involves forming combinations of three receivers and calculating the position using the analytical method. The total number of subsets is equal to the total number of combinations possible while choosing three receivers. The position estimates from each subset are combined to obtain a single position. There are many methods to combine the position estimates. The average of the positions will include any outlier and may not provide the best position estimate. 


\subsubsection{Angle of Arrival}

Angle of arrival (AoA) is different from the rest of the techniques because it consists of determining the intersection of the angles between the receiver antennae array and a transmitter rather than the intersection of the spheres. The ToF is measured between receivers in an array and the angle is calculated using the distance between the receivers. For a $2 \mathrm{D}$ solution two receivers are required but three receivers are required for a $3 \mathrm{D}$ solution. This technique is known as triangulation because the angle is used for localization instead of the distance. Angle of arrival works well for short distances but as the distance increases a small error in angle can result in a large position error.

\subsection{Lateration Experimental Work}

The selected lateration techniques for this thesis are analytical trilateration and LLS. Analytical trilateration is tested because there is limited space on the quadrotor for receivers and it only requires three. LLS is tested to see if adding more receivers can increase the position accuracy. Both of these methods provide fast update rates.

Therefore, to summarize, the results presented in this thesis are based on ultrasonic localization using ToF ranging with threshold detection. Either analytical trilateration or LLS are used for lateration. The following section details relevant literature introducing early work and similar techniques which are previously introduced.

\subsubsection{Early Work}

One of the earliest GPS-denied lateration localization systems is considered the active badge location system developed by AT\&T [34] for indoor localization. The system 
consists of employees wearing a badge that emits pulse width modulated infrared signals for approximately 0.1 s every 15 seconds. Each badge has a unique code to be able to distinguish the employees. The detection range is approximately 6 metres which allows an entire room to be covered while having a short enough range to not allow propagation into the neighboring rooms. This system does not have multiple receivers in the room to be able to perform trilateration but simply detects which room the employee is in.

A few years later AT\&T [35] developed another system using radio frequency (RF) and ultrasound referred to as the Bat system. A base station sends a RF signal to a unique Bat unit and it transmits an ultrasonic pulse. Receivers are placed at known locations throughout the building and if an ultrasonic transmission reaches a certain threshold the time of flight ( $\mathrm{ToF}$ ) is calculated. If the signal reaches more than three receivers the position is calculated through multilateration, a stationary accuracy of $9 \mathrm{~cm}$ with $95 \%$ confidence is obtained. If multiple bat units are placed on a single object, the orientation can also be calculated. Reverberations take up to $20 \mathrm{~ms}$ to die away which limits the update rate for the next bat to transmit its signal. Each transmitter is separated by a time delay to avoid interference, this is considered time division multiple access (TDMA). The cricket system [36] is the next key development for indoor localization, which is very similar to the bat system, but the localization is performed on a mobile device which improved privacy issues.

\subsubsection{Ultrasonic Localization}

There are only a few authors who use ultrasound for localization of a quadrotor, this is most likely due to the range limitation and complexity versus tracking a ground robot. Eckert [37, 38, 39] performs the simultaneous indoor localization of a quadrotor and multiple ground robots using ultrasound. Eckert uses multiple robots which have the capability to transmit and receive ultrasonic signals to allow for self-localization and 
the localization of other ground or aerial robots, the signals being modulated through TDMA. By using many self localization robots, Eckert is able to create a network for indoor localization. Eckert notes that, the issue with many localization methods for a quadrotor is the real-time localization aspect. A quadrotor requires fast position updates $(<5 \mathrm{~Hz})$ due to the vehicle dynamics while many of the previously discussed methods are too computational slow for use on a quadrotor. To determine the TOF threshold detection is used. Eckert favoured forming subsets rather than performing least squares. Eckert developed a Kalman type approach to predict the position from given subsets. Since all sensors are mounted on the same $\mathrm{Z}$ axis plane, the height of the drone responds drastically to small errors causing what is known as poor position dilution of precision (PDoP). Eckert uses additional sonar sensors to determine the height of the drone to an accuracy of $\pm 2 \mathrm{~cm}$. Vibrations from the motors carried through the frame are damped by suspending the beacon beneath the drone using cables. Eckert has a single beacon located on the quadrotor making localization only possible if there are four nearby ground robots. The maximum stationary variance of the system is $2 \mathrm{~cm}$, there is no in flight accuracy data recorded.

Parades [40] localizes a quadrotor indoors using ultrasound with five beacons mounted in the ceiling. Parades uses TDoA with cross correlation detection and nonlinear least squares to determine the position of the quadrotor. Since each beacon produces an ultrasonic signal they avoid interference through a combined time division and code division multiple access approach which leads to an average $2.4 \mathrm{~Hz}$ update rate. Similar to Eckert, Paredes tests a different approach which uses the ultrasonic system to detmine position $(\mathrm{x}, \mathrm{y})$ and to estimate the height through the use of a ToF camera which Parades calls the hybrid system. For stationary positioning Parades compares the hybrid system and using the ultrasonic to determine position and height. The hybrid system has a 3D stationary position standard deviation of $0.37 \mathrm{~cm}$ and the ultrasonic system of $1.58 \mathrm{~cm}$, an average accuracy increase of $76 \%$. Parades only 
presents stationary positioning data without any flight data.

Katona [41] investigates indoor localization for quadrotors using ultrasound. Katona uses the Marvelmind ultrasonic positioning system (discussed later) for localization. Katona notices that indoors the magnetometers tend to drift and can cause issues for control. To correct for this Katona places two receivers on the quadrotor such that it can detect the orientation to replace the magnetometer. Katona performs computer simulations for indoor localization but does not provide any experimental accuracies.

Other experiments use ultrasonic localization on ground robots, such as Nguyen [42]. Nguyen uses ToF with a sine wave detection algorithm to decrease the computational requirements of the cross-correlation detection methods. For stationary distance measuring an average error of $0.218 \mathrm{~cm}$ is obtained. Using five receivers, Nguyen compares the static LLS solution with the nonlinear least squares solution with a $40 \mathrm{~Hz}$ update rate. The LLS solution has an average error of $1.33 \mathrm{~cm}$ and the nonlinear least squares has an average error of $1.20 \mathrm{~cm}$ showing a $10.8 \%$ increase in accuracy. For dynamic testing around a circle a maximum distance error below $5 \mathrm{~cm}$ is achieved. However, this is a $2 \mathrm{D}$ solution with fixed receivers in the ceiling and a transmitter on the robot.

There are other experiments performed where ultrasonic localization is used not for control but simply tested on a moving object. Martin [33] uses ultrasonic ToF subset generation with threshold detection for indoor non line of sight localization. Martin organizes subset positions in order and finds the median value. Martin then averages the values within one standard deviation of the median value to find an estimate of the true position. This approach is efficient at rejecting outliers while still averaging multiple measurements. Martin tests dynamic positioning data by moving the beacon on a robotic arm, Martin obtains a maximum positioning error of less than $0.5 \mathrm{~mm}$. Qi [43] uses the Hilbert transform to determine the envelope for peak 
detection and uses linear least squares for multilateration. Qi obtains a maximum distance error of $1.42 \mathrm{~mm}$ at a distance of $3.151 \mathrm{~m}$. Qi obtains a maximum lateral position accuracy of $10.2 \mathrm{~mm}$ on a moving ground based robot using a Vicon system with $1 \mathrm{~mm}$ accuracy as truth data.

Other experiments on stationary objects have obtained high accuracy using ultrasonic localization. Saad [32] obtains range measurements using cross correlation featuring frequency hop spread spectrum. Saad improves his accuracy by using the phase shift of the signal. Saad obtains a distance accuracy of $0.5 \mathrm{~mm}$ with $90 \%$ confidence. Saad does not perform lateration to calculate the position. Medina [44] performs ultrasonic localization using ToF with phase of arrival, envelope detection and LLS for lateration. Medina uses quadrature detection to determine the envelope of the wave and phase shift to obtain a root mean square error less than $2 \mathrm{~mm}$ for the ToF measurements. For positioning Medina uses LLS and obtains a stationary RMSE of $9.6 \mathrm{~mm}$. De Angelis [45] performs localization with ultrasound using ToF with cross correlation detection and LLS for positioning. A stationary position standard deviation of $0.8 \mathrm{~mm}$ is obtained at a distance of $4 \mathrm{~m}$. Bischoff [46] uses angle of arrival and TDoA with threshold detection for ultrasonic indoor localization. For stationary position Bischoff obtains an average standard deviation of $0.57 \mathrm{~cm}$. Khyam [47] uses phase of arrival to augment the TDoA measurement captured using a correlation method for indoor ultrasonic localization and LLS for positioning. Khyam obtains a RMSE stationary position accuracy of $6.12 \mathrm{~mm}$.

\subsubsection{Other Localization Methods}

Several authors have used ultra wideband localization for quadrotors or ground robots, which is an improvement over ultrasonic due to its increased range. Benini [48] performs indoor localization of a quadrotor using Ubisense UWB technologies. It operates using TDoA ranging, threshold detection and an EKF for lateration. The 
system is capable of a stationary distance accuracy of $15 \mathrm{~cm}$ with $95 \%$ confidence. The maximum distance is $160 \mathrm{~m}$ and maximum update rate is $10 \mathrm{~Hz}$. There is no accuracy calculated using in flight data.

Raja [49] performs UWB indoor localization of a quadrotor using TDoA ranging technique but does not discuss the lateration technique. A standard deviation of stationary ranging distances of $14 \mathrm{~mm}$ is achieved. A stationary position standard deviation of $0.538 \mathrm{~mm}$ is achieved. This is implemented on a Parrot drone but there is no dynamic data presented. Segura [50] performs UWB localization of a ground robot using TDoA ranging, threshold detection and a least squares approach. Segura obtains a stationary lateral RMSE of $10.3 \mathrm{~cm}$. Segura performs indoor localization using a ground robot and received errors less than $20 \mathrm{~cm}$ with a $95 \%$ confidence.

Kirchner [51] uses IR for localization of indoor UAVs, Kirchner uses diffuse IR with the emitter located on the UAV. The receivers measure the incoming angle and calculate the position using triangulation. The system has a maximum stationary error of $1.6 \mathrm{~cm}$ between $1 \mathrm{~m}-2 \mathrm{~m}$, outside this range the error increases. A position is calculated $93 \%$ of the time for dynamic flight testing, however no associated accuracies are reported.

Lategahn [52] performs WiFi localization of a target using both TDoA and received signal strength ranging techniques with an EKF for indoor localization. By fusing both estimates together Lategahn is able to get a more accurate position than using TDoA alone. A person walked in a rectangular grid and using TDoA alone an average error of $1.5 \mathrm{~m}$ is obtained and by fusing both methods an average error if 1.0 $\mathrm{m}$ is obtained. This system is not connected to a control system.

Paterna [53] uses bluetooth localization of a ground robot with received signal strength and uses a weighted trilateration algorithm to determine the position. The stationary position accuracy is $70 \mathrm{~cm}$ with $90 \%$ confidence. The position accuracy using a moving ground robot is $182 \mathrm{~cm}$ with $90 \%$ confidence. 
Zhang [54] performs large scale RFID localization of a target using active RFID tags with received signal strength ranging and interpolation between tags to determine the position. A stationary position accuracy of up to $30 \mathrm{~cm}$ is achieved over a distance of $1000 \mathrm{~m}$. There is no dynamic data available. This system is not connected to a control system.

\subsubsection{Commercial Systems}

There are a few commercial systems which offer localization in a GPS-denied environment. The selected systems are the most popular using their localization method.

Optitrack [55] uses vision-based localization, the basic system consists of 4 cameras which operate at 100 FPS and have a resolution of $640 \times 480$. This system is capable localization of up to 4 quadrotors in a $2 \mathrm{~m}$ diameter circle and a height of $2 \mathrm{~m}$. The required setup area for this is $6 \mathrm{~m}$. This system can provide a position error of less than $0.3 \mathrm{~mm}$. Upgrades can increase the quantity and quality of the cameras to increase area up to $15 \times 15 \times 6$ m, 360 FPS, $2048 \times 2048$ resolution and track up to 48 drones. Vicon motion capture is another vision-based localization company which offer similar services with similar accuracy.

Marvelmind [28] is an ultrasonic localization system which offers 4 beacons (receivers) and 1 mobile Hedgehog (transmitter) for $\$ 400$ USD. It claims a 2 cm stationary distance accuracy with a maximum update rate of $45 \mathrm{~Hz}$. It does not provide a position accuracy because that depends on the geometry of the receivers setup. The maximum range without noise is $30 \mathrm{~m}$. It uses threshold detection with TDoA.

Pozyx [56] is an UWB localization company that offers a tag (transmitter) and four anchors (receivers) for $\$ 600$ USD. It offers $10 \mathrm{~cm}$ stationary distance accuracy, an update rate of $140 \mathrm{~Hz}$ and a typical range of $30 \mathrm{~m}$. In an open environment the range can reach up to $100 \mathrm{~m}$. There is no position accuracy offered because it depends on the geometry of the receivers. There is an IMU within the tag which is integrated into 
the position measurement through a Kalman filter to provide more accurate position information. The system uses threshold detection and TDoA. The system typically uses non-linear least squares but offers other techniques for testing or research.

\subsubsection{Summary}

A summary of the experimental results are provided in Table 2.2. The application refers to whether it is used for control for a flying robot, a ground robot or if is just tracking the target and not used for control purposes.

The system designed in this thesis uses ultrasonic localization with ToF ranging, threshold detection and for lateration LLS or analytical trilateration. The other reviewed flying applications do not use this configuration, Eckert [57] uses subsets instead of LLS for lateration. Eckert achieves the best stationary accuracy for flying applications with a stationary maximum error of less than $2 \mathrm{~cm}$. For his application Eckert includes possible non line of sight localization which introduces outliers and LLS does not handle outliers well. For this thesis it is limited to line of sight localization only. Parades [40] uses a more complicated setup with cross correlation detection and nonlinear least squares which achieves a stationary standard deviation of $3.7 \mathrm{~cm}$ but at a maximum update rate of $2.4 \mathrm{hz}$. For this thesis a simpler approach is taken which should have a worse accuracy but a better update rate.

This localization configuration has been used in earlier work by AT\&T which uses the system to track a target on a person with a $9 \mathrm{~cm}$ accuracy (95\% confidence). This is the expected accuracy of the system, which is sufficient for this application as it is more accurate than the $1.891 \mathrm{~m}$ (95\% confidence) horizontal GPS accuracy as previously mentioned [1].

Similar ultrasonic configurations have potential for higher accuracy, the most accurate system tested on a moving target is Qi [43] who uses the same configuration 
except envelope detection instead of threshold detection. Qi is able to obtain a maximum stationary error of $1.42 \mathrm{~mm}$ and a maximum moving error of $10.2 \mathrm{~mm}$ on a moving ground robot. The speed of the ground robot and the update rate are not provided but based on similar configurations the update rate is low due to computational requirements.

Nearly all of the researched ultrasonic systems use a single beacon on a target (person, robot, quadrotor) and place multiple receivers at fixed locations around the target. This limits localization to areas where the fixed receivers are located. Eckert presents the only work which places the multiple receivers on moving robots to create a moving framework. However this still requires fixed receivers to localize the ground robots as their internal IMU drifts over time. Rather than placing the movable beacon on the quadrotor, this work places the fixed receivers on the quadrotor and allows the beacon to be portable. This approach has not been encountered in the literature review.

Wang [27] is able to perform the same applications as this thesis using a vision system. Wang is able to achieve an average error in hover of less than $2 \mathrm{~cm}$ for a stationary target and less than $3 \mathrm{~cm}$ for a moving target. Vision systems present an alternative approach to the problem but require higher computational power, in Wang's application the image processing is performed on an off flight platform computer which is connected over WiFi. In this thesis the ultrasonic localization system is all on board and does not require an external computer. 


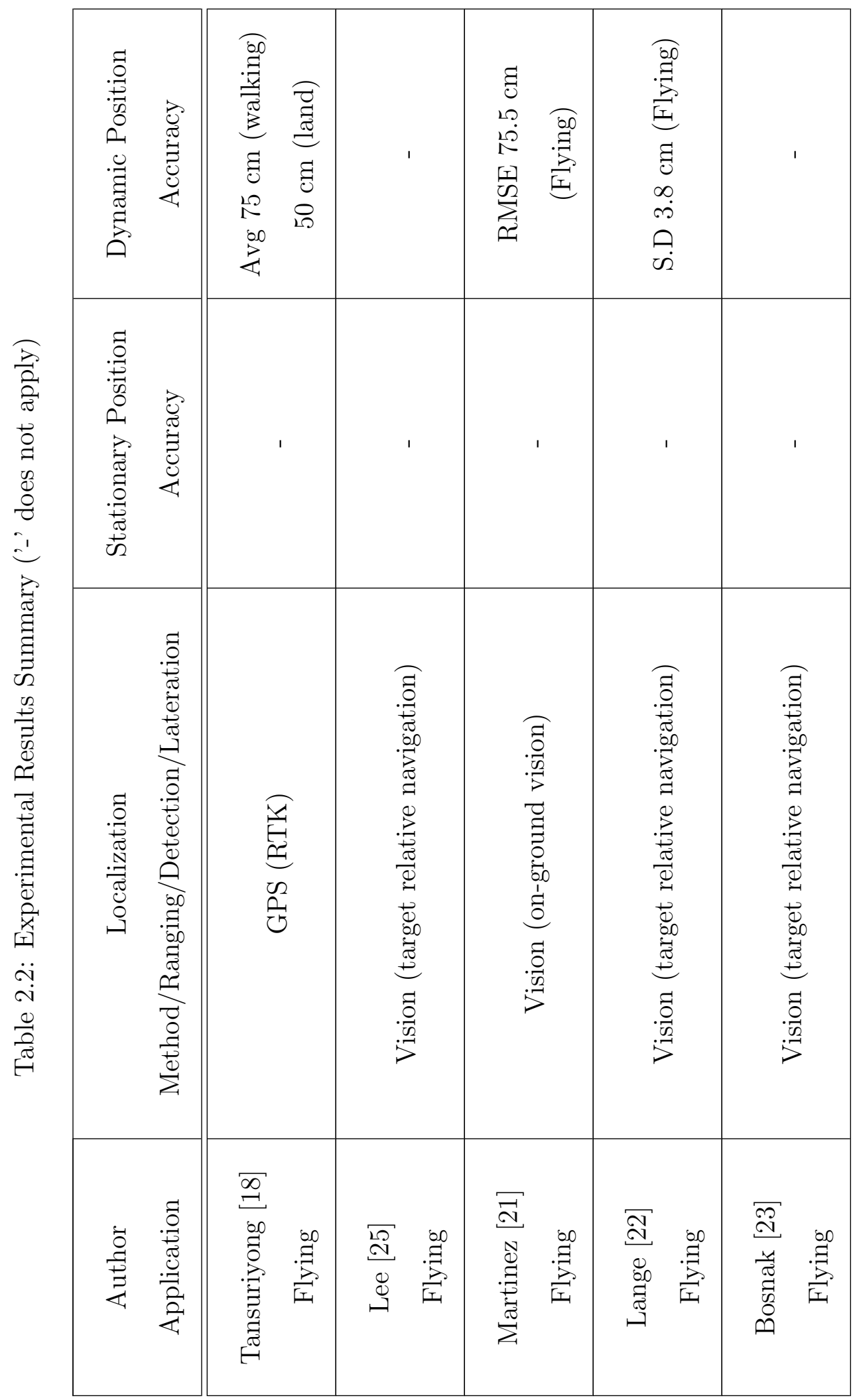




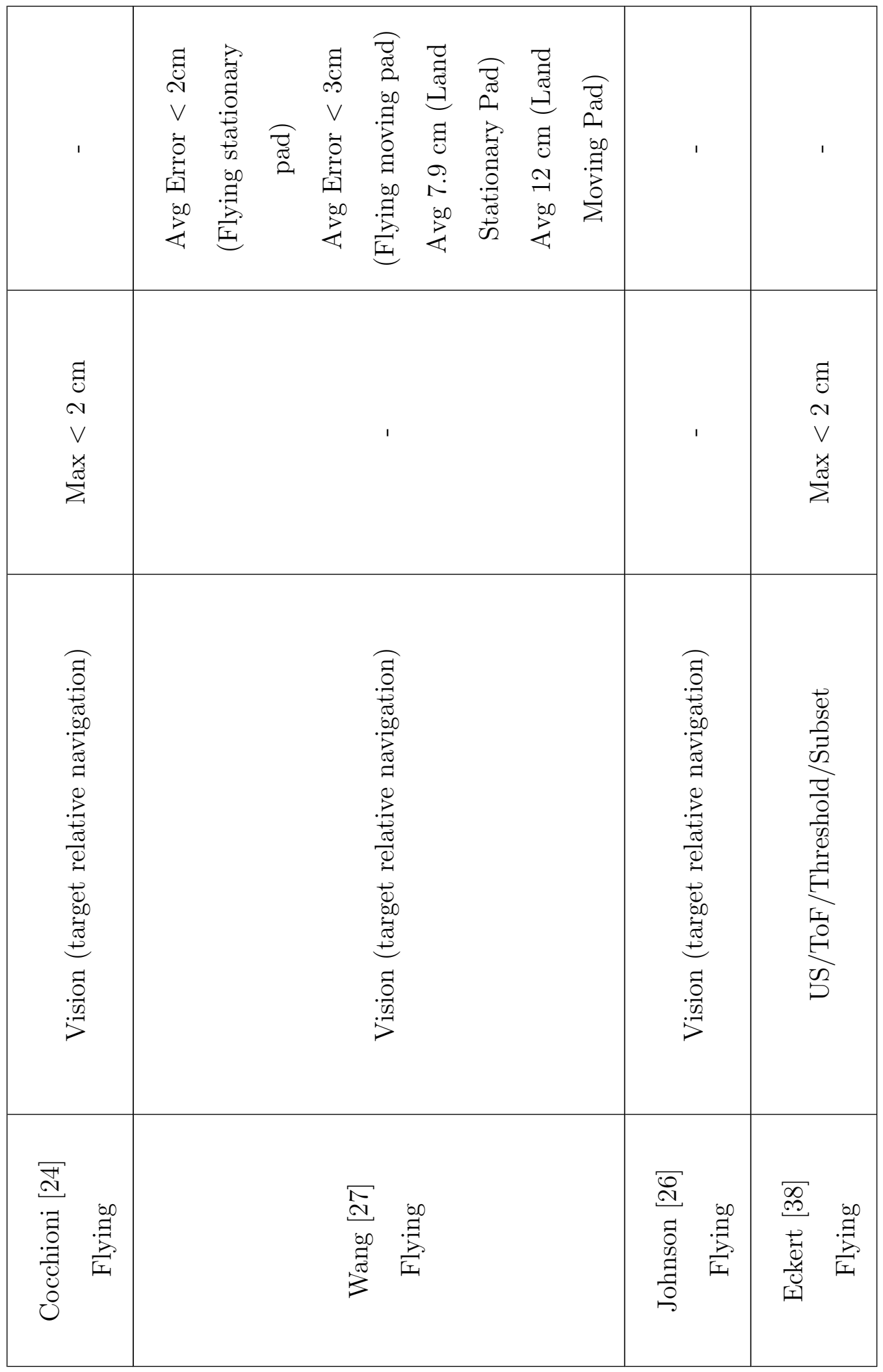




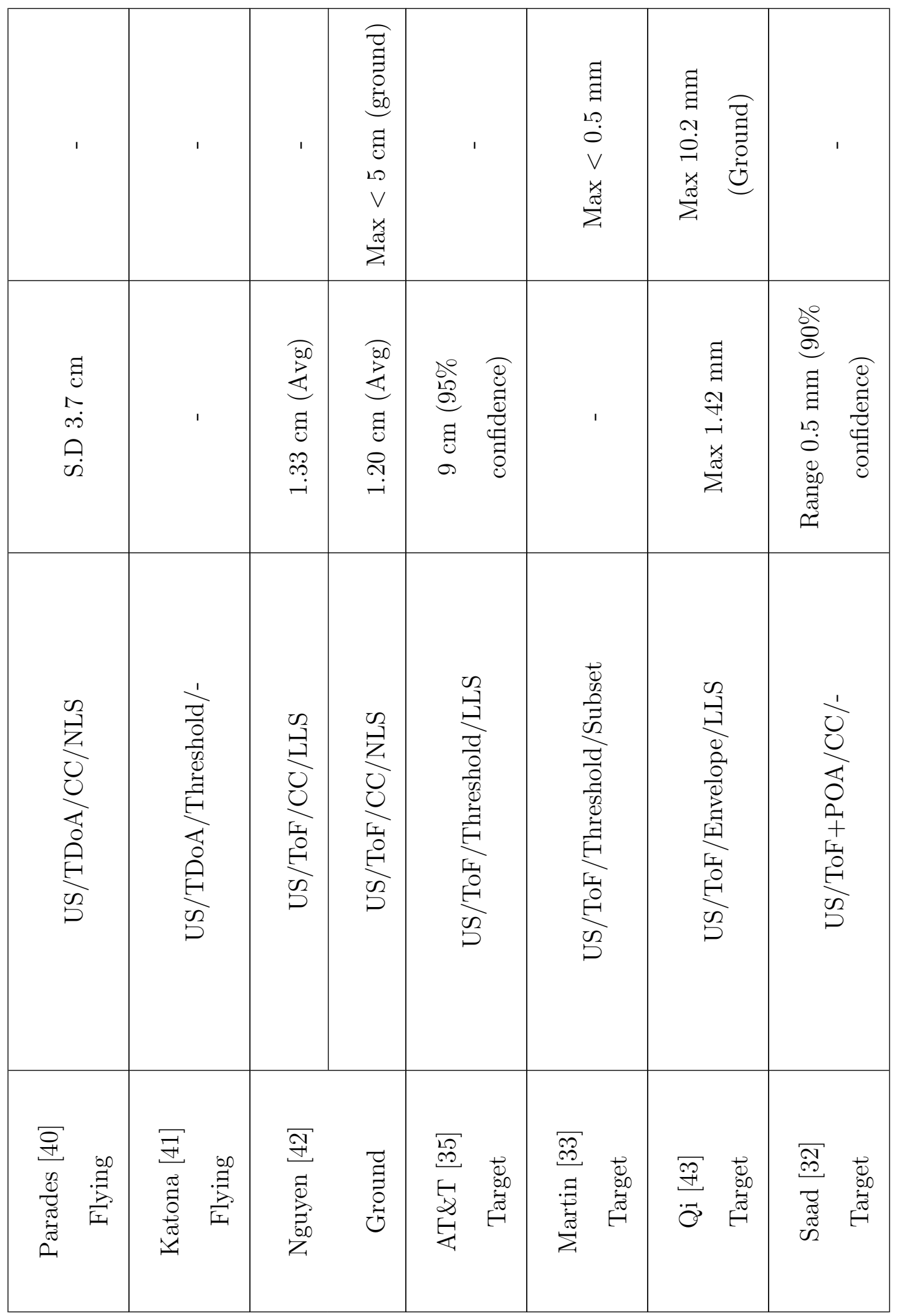




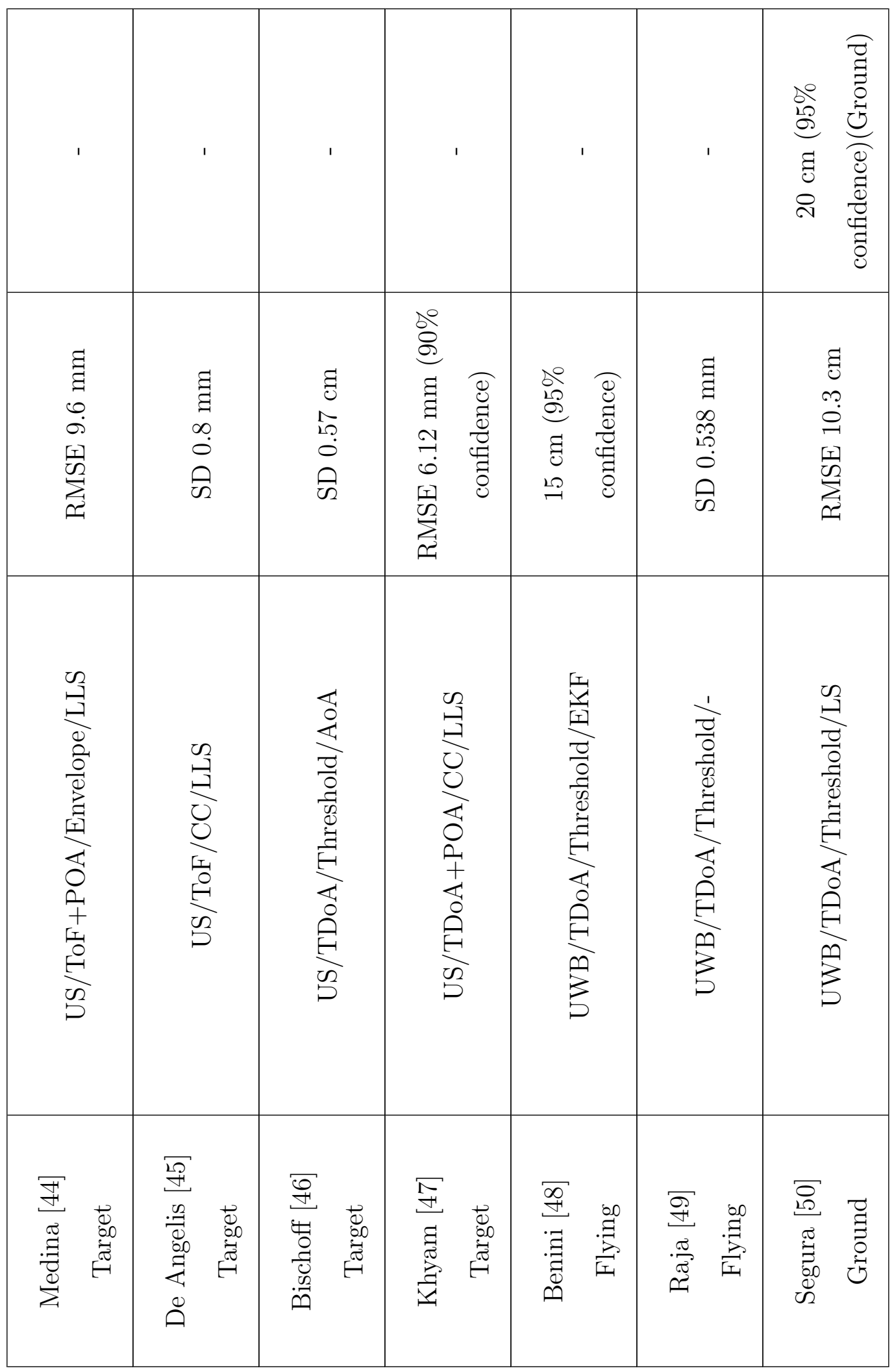




\begin{tabular}{|c|c|c|c|c|c|c|c|}
\hline & 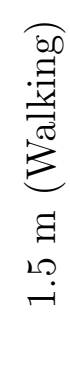 & 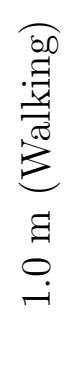 & 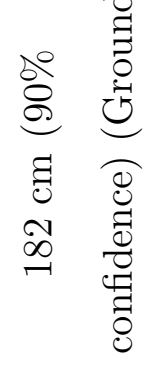 & ' & 1 & 1 & ' \\
\hline $\begin{array}{l}\text { व્ } \\
\text { o } \\
\stackrel{-}{-}\end{array}$ & 1 & ' & 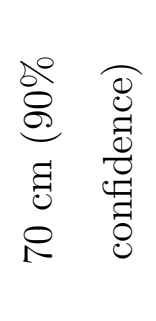 & 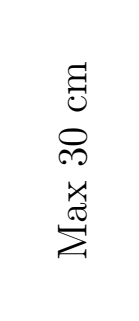 & 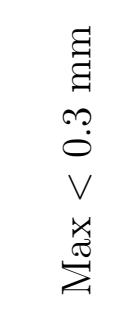 & 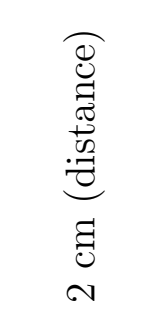 & 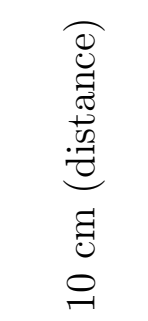 \\
\hline 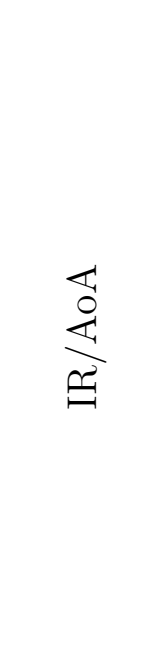 & 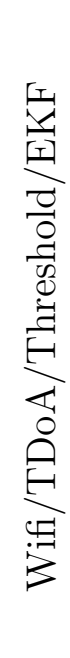 & 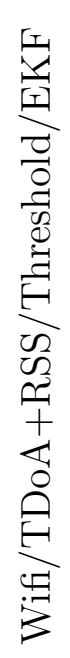 & 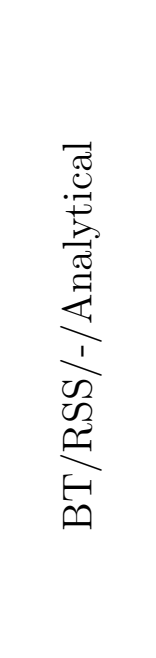 & 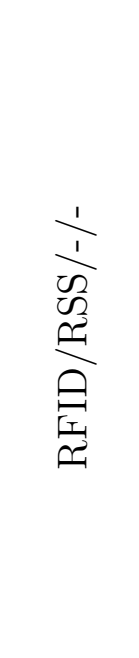 & 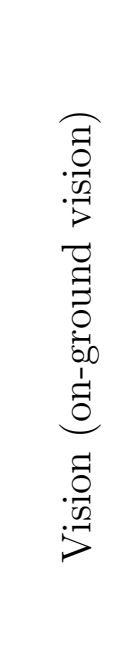 & 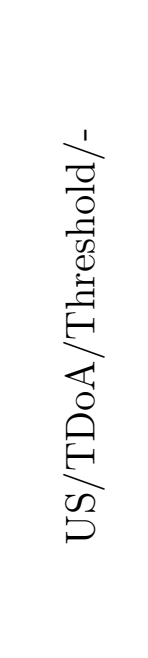 & 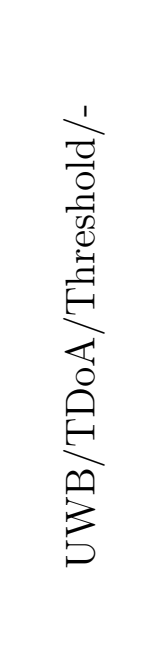 \\
\hline 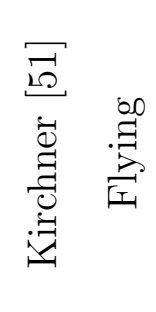 & 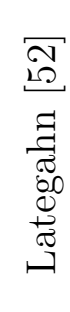 & 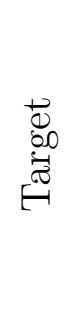 & 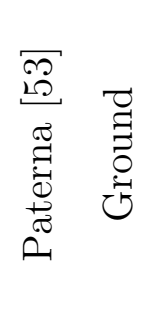 & 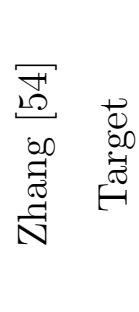 & 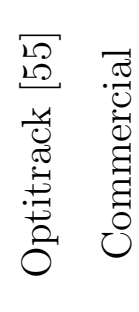 & 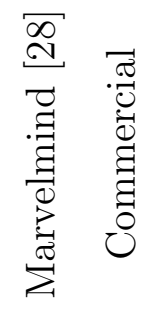 & 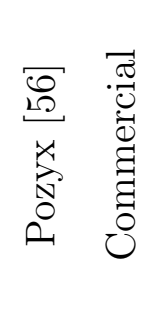 \\
\hline
\end{tabular}




\section{Chapter 3}

\section{Flight Vehicle}

The quadrotor used as a test platform is shown in Fig. 3.1 and is named TARA 3. TARA 3 is $58 \mathrm{~cm}$ wide but has a wingspan of $80 \mathrm{~cm}$ from corner to corner. TARA 3 is an aluminum framed quadrotor which uses a Pixhawk running ArduCopter (3.5.7) software for control. The piezo electric transducer's, arm extensions and Programmable System on Chip (PSoC) are part of the ultrasonic localization system (ULS). The ULS is not necessary for the flight vehicle and will be discussed in the next chapter. ArduCopter interfaces with other devices through Micro Aerial Vehicle Link (MAVLink) which limits what devices it can connect to. It is unable to directly connect with the ultrasonic localization system (ULS), therefore a Raspberry Pi 3 (RPi) provides an interface between Arducopter and the ULS. TARA 3 must be able to hover and land using the ULS in a GPS denied environment and a real world outdoor environment.

Next the flight vehicle is divided into two main sections, the sensors, and the software components. 


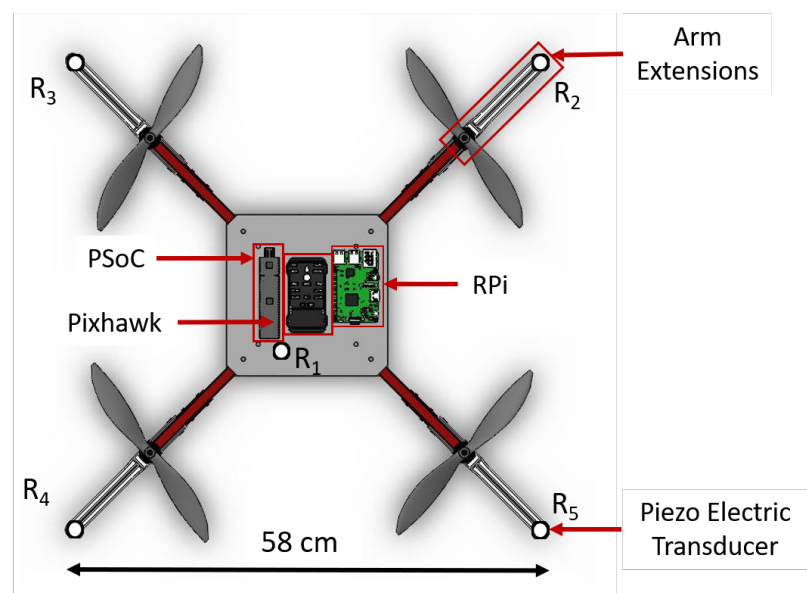

Figure 3.1: TARA 3 layout

\subsection{Sensors}

The Pixhawk is the main hardware component which contains several sensors and provides a connection interface for external sensors. The Pixhawk contains an inertial measurement unit (IMU) for attitude stabilization. The IMU consists of an accelerometer, gyroscope, and magnetometer for each axis. The Pixhawk also contains a barometer for a relative altitude measurement. For position and velocity estimation typically a GNSS module is used, but for GPS denied environments, the ULS performs this function.

\subsubsection{Pixhawk}

Pixhawk is an open-hardware autopilot which can be used to control fixed wing aircraft, ground robots, and many other platforms. The Pixhawk communicates with companion computers using MAVlink messages. The technical specification for the Pixhawk are as follows [12]:

\section{Processor}

- 32bit STM32F427 Cortex M4 core with FPU, 168 MHz, 256 KB RAM, 2 MB 
Flash

- 32-bit STM32F103 failsafe co-processor

\section{Internal Sensors}

- Invensense MPU 6000 16-bit accelerometer/gyroscope (Main) [58]

- ST Micro L3GD20H 16-bit gyroscope [59]

- ST Micro LSM303D 14-bit accelerometer / magnetometer [60]

- MEAS MS5611 barometer [61]

The Pixhawk uses two accelerometers and two gyroscopes which are made by different companies. Having multiple sensors provides reliability in case one sensor fails in flight, the vehicle can still be landed using the other sensor for control. The Pixhawk determines if a sensor has failed by comparing the sensor measurements to the other sensor and an EKF prediction, if the sensor has too large of variance it may require re-calibration or replacement. By using sensors by different manufacturers it decreases the chances of them failing for the same reason as each brand is constructed differently. While both sensors are working a more accurate measurement is obtained by fusing the measurements within an internal EKF in the Arducopter software.

\section{IMU}

The IMU contains a three-axis accelerometer to measure accelerations along each axis, a three-axis gyroscope to measure angular rates along each axis, and a threeaxis magnetometer to measure the magnetic field strength along each axis. These measured accelerations can be integrated over time to determine relative velocities and a relative position of the quadrotor. The angular rates are integrated to determine the attitude of the quadrotor. The issue with integration is that the velocity, 
position, and attitude accumulate errors over time and require corrections from other instruments. The Pixhawk samples these measurements at a maximum of $100 \mathrm{~Hz}$. The magneteic field measurements can be used to determine the yaw of the quadrotor relative to magnetic North. The Pixhawk uses a maximum update rate of $14 \mathrm{~Hz}$ for the magnetometer [62].

\section{Barometer}

The barometer measures atmospheric pressure, the change in atmospheric pressure can be related to the change in altitude. This pressure is not used to determine absolute altitude but instead relative altitude. The time rate of change of the relative altitude can be used to determine the vertical velocity. The Pixhawk uses a MS561101BA03 barometer which has a resolution of $10 \mathrm{~cm}$ [61] and updates at a maximum rate of $14 \mathrm{~Hz}[62]$.

\subsubsection{GNSS}

A GNSS module is used for comparison to the ULS. The Neo-M8N GNSS can access GPS, GLONASS, BeiDou and Galileo systems as well as simultaneously accessing GPS and GLONASS for increased satellite coverage. The reported specifications are provided in Table 5.1.

\subsection{Software}

The software is broken down into Arducopter, RPi Position controller and complete quadrotor control schemes to allow these to function. 
Table 3.1: GPS Accuracy

\begin{tabular}{|c|c|c|}
\hline Parameter & Reported [63] & Units \\
\hline \hline Accuracy & 250 (Absolute) & $\mathrm{cm}$ \\
\hline Frequency & $<14$ & $\mathrm{~Hz}$ \\
\hline Velocity Accuracy & 5 & $\mathrm{~cm} / \mathrm{s}$ \\
\hline Heading Accuracy & $0.3^{\circ}$ & $\mathrm{N} / \mathrm{A}$ \\
\hline
\end{tabular}

\subsubsection{Arducopter}

ArduCopter is an open source code developed and maintained by DIY Drones. The code is responsible for attitude stabilization and is designed for autonomous control through GPS waypoints. The code can be operated in one of several flight modes,

A Altitude Hold: This mode is used for manual takeoff and positioning the quadrotor into the desired starting position, after which it is switched into one of the other modes depending on the mission. This mode is designed to stabilize the desired attitude and with the throttle position in neutral a quadrotor will maintain its altitude. Increasing or decreasing the throttle will cause the quadrotor to ascend or descend respectively. The user controls the desired attitude through an RC transmitter. This mode does not require GPS.

B GPS Hold: This mode is used for GPS hovering. It maintains the current GPS position through a position PID controller. The position controller provides desired attitude inputs into the attitude controller. The desired yaw angle is set to the current yaw angle when this mode is initialized. This mode is not 
used in this thesis but is featured as a comparison in Appendix B.

C Land: This mode is used for GPS landing. Lands at the current location using a set a downward velocity of $50 \mathrm{~cm} / \mathrm{s}$. It will control its lateral position using the same controller as GPS Hold. The desired yaw angle is set to the current yaw angle when this mode is initialized. This mode is not used in this thesis but is featured as a comparison in Appendix B.

D Guided No GPS: This mode is used for ULS hovering and landing. It allows for autonomous control through desired attitude commands, while ArduCopter stabilizes the desired attitude. The desired vertical velocity must be specified and the desired yaw angle is set to the current yaw angle when this mode is initialized.

Arducopter has an EKF which is used to combine measurements from the sensors to estimate the aircraft state. When a GPS is connected the EKF can provide accurate estimates of position, $\left(x_{E}, y_{E}, z_{E}\right)$, and velocities, $\left(\dot{x}_{E}, \dot{y}_{E}, \dot{z}_{E}\right)$. This allows for position control for the GPS Hold and Land mode. Without the GPS, there is no reliable position or velocity data for feedback control. However it still produces accurate attitude $\left(\phi_{E}, \theta_{E}, \psi_{E}\right)$, angular rates, $\left(\dot{\phi}_{E}, \dot{\theta}_{E}, \dot{\psi}_{E}\right)$, and angular accelerations $\left(\ddot{\phi}_{E}, \ddot{\theta}_{E}, \ddot{\psi}_{E}\right)$. This is the reason Guided no GPS requires an attitude rather than a position. Through the barometer the vertical velocity, $\dot{z}_{E}$, can still be calculated which allows the vertical velocity to be specified.

\subsubsection{Raspberry Pi Position Controller}

A RPi 3 is used for the position control to interface with the Pixhawk. The RPi receives the position from the ULS through $I^{2} C$ protocol. The RPi connects with the Pixhawk through UART protocol and communicates to ArduCopter through 
MAVlink messages. The main control loop runs at $25 \mathrm{~Hz}$ which is greater than the position inputs from the ULS at $10 \mathrm{~Hz}$. This frequency surpasses the Nyquist criterion and ensures that the position data is current. The old position data is used for control when new data is unavailable.

The RPi receives positions, $\left(x_{k}, y_{k}, z_{k}\right)$, and velocities $\left(\dot{x}_{k}, \dot{y}_{k}, \dot{z}_{k}\right)$ from the ULS. It must convert these positions and velocities into desired roll and pitch angles $\left(\phi_{d}, \theta_{d}\right)$, and a desired vertical velocity $\dot{z}_{d}$. It performs this through a position PID controller which takes in the position error and calculates a desired attitude and desired vertical velocity. The position error is calculated based on the ULS position and the desired position $\left(x_{d}, y_{d}, z_{d}\right)$. For hover, the desired location is above the beacon at a desired hover height, this is represented by $\left(0,0, z_{d}\right)$. For landing it follows a similar principle but the desired height, $z_{d}$, continually decreases until it reaches the ground. The control scheme is shown in Fig. 3.2.

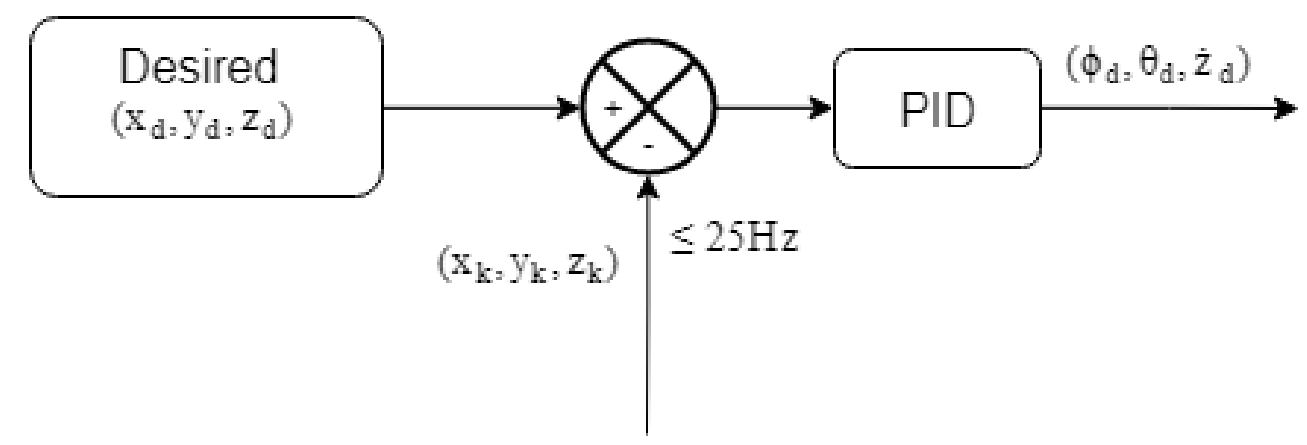

Figure 3.2: RPi position control block diagram

For the quadrotor to move forward along the $\mathrm{X}$ axis, it must pitch forward. Similarily, for the quadrotor to move forward along the $\mathrm{Y}$ axis, it must roll right. A desired pitch and roll angle is calculated based on the position error and the velocity error. The position, $\left(x_{k}, y_{k}\right)$, is measured through the ULS. 


$$
\begin{gathered}
\theta_{d}=K_{P_{X}} \underbrace{\left(x_{d}-x_{k}\right)}_{\text {position error }}+K_{I_{X}} \int_{0}^{t}\left(x_{d}-x_{k}\right) d t+K_{D_{X}} \frac{d\left(x_{d}-x_{k}\right)}{d t} \\
\phi_{d}=K_{P_{Y}} \underbrace{\left(y_{d}-y_{k}\right)}_{\text {position error }}+K_{I_{Y}} \int_{0}^{t}\left(y_{d}-y_{k}\right) d t+K_{D_{Y}} \frac{d\left(y_{d}-y_{k}\right)}{d t}
\end{gathered}
$$

The altitude error is converted into a desired velocity, $\dot{z}_{d}$,

$$
\dot{z}_{d}=K_{P_{Z}} \underbrace{\left(z_{d}-z_{k}\right)}_{\text {altitude error }}+K_{I_{Z}} \int_{0}^{t}\left(z_{d}-z_{k}\right) d t+K_{D_{Z}} \frac{d\left(z_{d}-z_{k}\right)}{d t}
$$

The gains for the position PID controllers are provided in Table 3.2. These gains are initially tuned using the ZieglerNichols $(\mathrm{Z}-\mathrm{N})$ method and then further tuned experimentally. The Z-N method is discussed in Section 3.2.2.

Table 3.2: Position Stabilization Gains

\begin{tabular}{|l|c|c|c|c|c|c|c|c|}
\hline$K_{P_{X}}$ & $K_{I_{X}}$ & $K_{D_{X}}$ & $K_{P_{Y}}$ & $K_{I_{Y}}$ & $K_{D_{Y}}$ & $K_{P_{Z}}$ & $K_{I_{Z}}$ & $K_{D_{Z}}$ \\
\hline \hline 0.50 & 0.050 & 4.0 & 0.50 & 0.050 & 4.0 & 0.017 & 0.030 & 0.060 \\
\hline
\end{tabular}

\section{Ziegler-Nichols Tuning}

Ziegler-Nichols (Z-N) is a PID tuning algorithm which estimates controller gains by measuring the ultimate gain, $K_{u}$, for loop stability and the period of oscillation, $T_{u}$, at the stability limit. It assumes the transfer function for the system follows the form given by:

$$
G(s)=\frac{K_{Z N} e^{-s t}}{\chi+s}
$$


This model predicts that there is one pole $(\chi)$ with a time delay $(t)$ to capture the amplitude roll off and the phase of the system. This simplification works well if the system is linear and the response is dominated by a single pole exponential lag [64]. According to the Nyquist stability criterion, the stability limit has a 180 degree phase shift. The first order pole contributes $90^{\circ}$ of phase shift while the rest of the phase is covered by the phase adjustment.

The Z-N method is used to tune the lateral position controller, provided in Eq. 3.1 and 3.2. To obtain the ultimate gain, $K_{u}$, and the period of oscillation, $T_{u}$, the gain of the system is increased until a steady oscillation occurs, at which point the stability limit has been reached. The gain at this point is then the ultimate gain, $K_{u}$, while the period of the observed oscillation is $T_{u}$. The PID gains can then be calculated according to the nominal values provided in Table 3.3.

This is performed by providing a position offset and allowing the quadrotor to move back and forth. The measured position versus time along each axis in the horizontal plane at the stability limit is shown in Fig. 3.3. As shown, the measured period is approximately $13 \mathrm{~s}$, which is obtained with a gain, $K_{u}$, of 1.30 . The PID gains are presented in Table 3.3 using the classical Z-N approach. Since the Z-N method approximates the system as a first order system the gains do not result in good control behaviour. Fine tuning is performed to obtain acceptable control behaviour.

\subsubsection{Control}

The control schemes are divided into different sections based on the flight mode, only the flight modes used in this thesis are discussed. The Guided No GPS mode is discussed first as it is a continuation from the RPi position control scheme. 


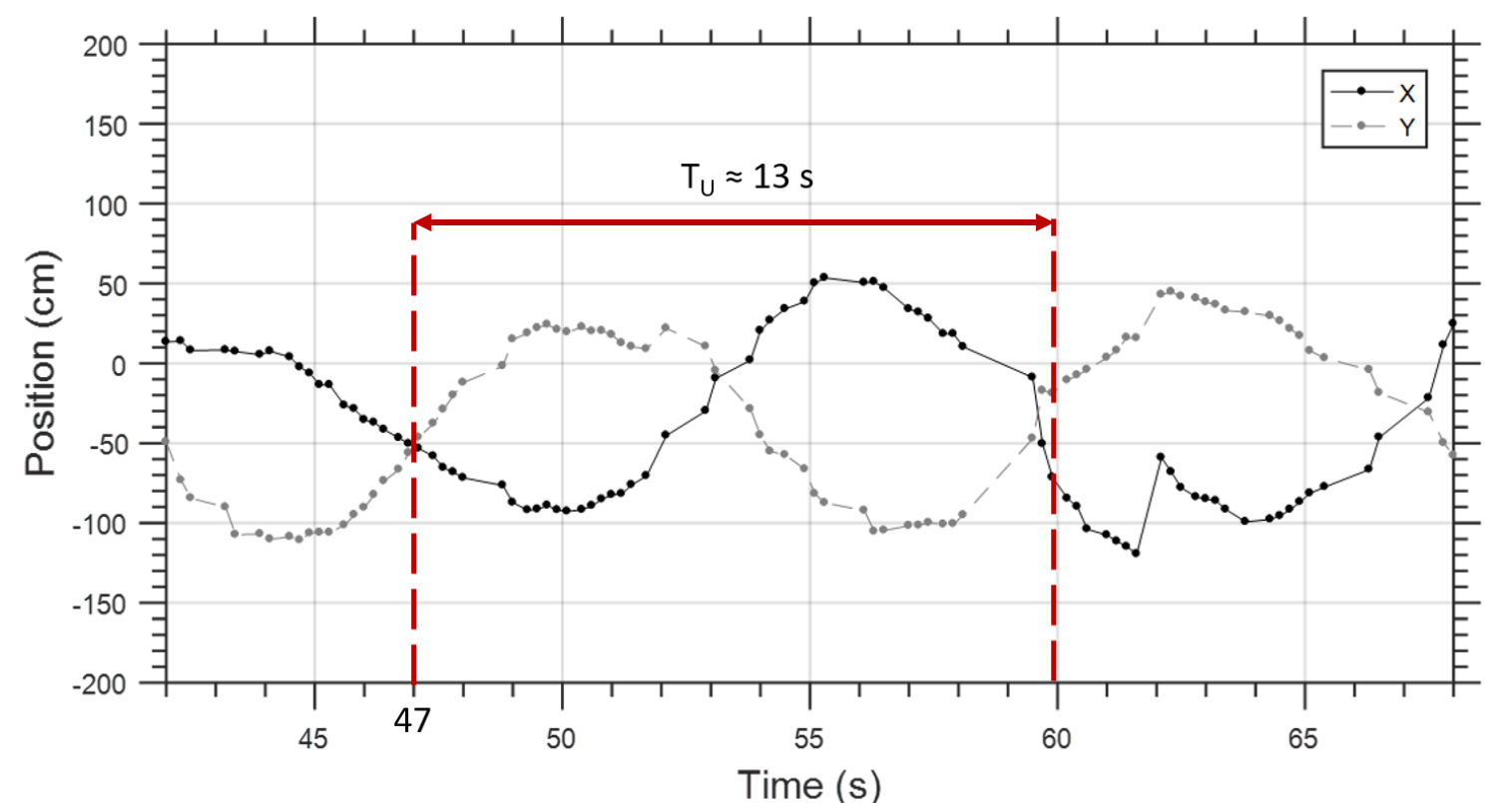

Figure 3.3: Experimental flight data for finding $K_{u}$ and $T_{u}$

\section{Guided No GPS}

Guided No GPS flight mode is selected while the ULS is used. This mode requires an external RPi controller because the ULS provides position and velocity data but this flight mode requires desired attitude inputs. The overall control scheme is shown in Fig. 3.4, where the RPi position controller has previously been discussed. This discussion will focus on the ArduCopter portion. The desired yaw angle is set to the current yaw angle when this mode is initialized. ArduCopter is responsible for stabilizing the desired attitude, $\left(\phi_{d}, \theta_{d}, \psi_{d}\right)$ and the desired vertical velocity, $\dot{z}_{d}$ provided by the RPi. The attitude stabilization will be discussed first followed by the altitude control.

The first controller is a proportional controller designed to stabilize the roll, pitch and yaw angles. The desired roll and pitch angles $\left(\phi_{d}, \theta_{d}\right)$ are calculated based off the position controller (Eq's 3.1-3.2). The current angles $\left(\phi_{E}, \theta_{E}, \psi_{E}\right)$ are calculated by the ArduCopter EKF. This proportional controller calculates the desired angular 
Table 3.3: Summary of PID Gains

\begin{tabular}{|c|c|c|c|}
\hline Gain & Nominal & Value & Fine Tuning \\
\hline \hline$K_{P}$ & $0.6 K_{u}$ & 0.72 & 0.50 \\
\hline$K_{I}$ & $\frac{1.2 K_{u}}{T_{u}}$ & 0.12 & 0.05 \\
\hline$K_{D}$ & $\frac{3.0 K_{u} T_{u}}{40}$ & 1.08 & 4.0 \\
\hline
\end{tabular}

rates, $\left(\dot{\phi}_{d}, \dot{\theta}_{d}, \dot{\psi}_{d}\right)$, based off the angular error to obtain the desired attitude. These control laws are shown in Eq's 3.5-3.7.

$$
\begin{aligned}
& \dot{\phi}_{d}=K_{P_{\phi}} \underbrace{\left(\phi_{d}-\phi_{E}\right)}_{\text {angular error }} \\
& \dot{\theta}_{d}=K_{P_{\theta}} \underbrace{\left(\theta_{d}-\theta_{E}\right)}_{\text {angular error }} \\
& \dot{\psi}_{d}=K_{P_{\psi}} \underbrace{\left(\psi_{d}-\psi_{E}\right)}_{\text {angular error }}
\end{aligned}
$$

A PID controller is implemented to stabilize the desired roll, pitch and yaw rates, $\left(\dot{\phi}_{d}, \dot{\theta}_{d}, \dot{\psi}_{d}\right)$, and produces motor outputs, $\left(U_{\phi}, U_{\theta}, U_{\psi}\right)$. The desired angular rates, $\left(\dot{\phi}_{d}, \dot{\theta}_{d}, \dot{\psi}_{d}\right)$, are calculated in the previous controller (Eq's 3.5-3.7). The current angular rates, $\left(\dot{\phi}_{E}, \dot{\theta}_{E}, \dot{\psi}_{E}\right)$ are calculated by the ArduCopter EKF. The control laws to stabilize the angular rates are shown in Eq. 3.8, Eq. 3.9, and Eq. 3.12.

$$
U_{\phi}=K_{P_{\dot{\phi}}} \underbrace{\left(\dot{\phi}_{d}-\dot{\phi}_{E}\right)}_{\text {rate error }}+K_{I_{\dot{\phi}}} \int_{0}^{t}\left(\dot{\phi}_{d}-\dot{\phi}_{E}\right) d t+K_{D_{\phi}} \frac{d\left(\dot{\phi}_{d}-\dot{\phi}_{E}\right)}{d t}
$$




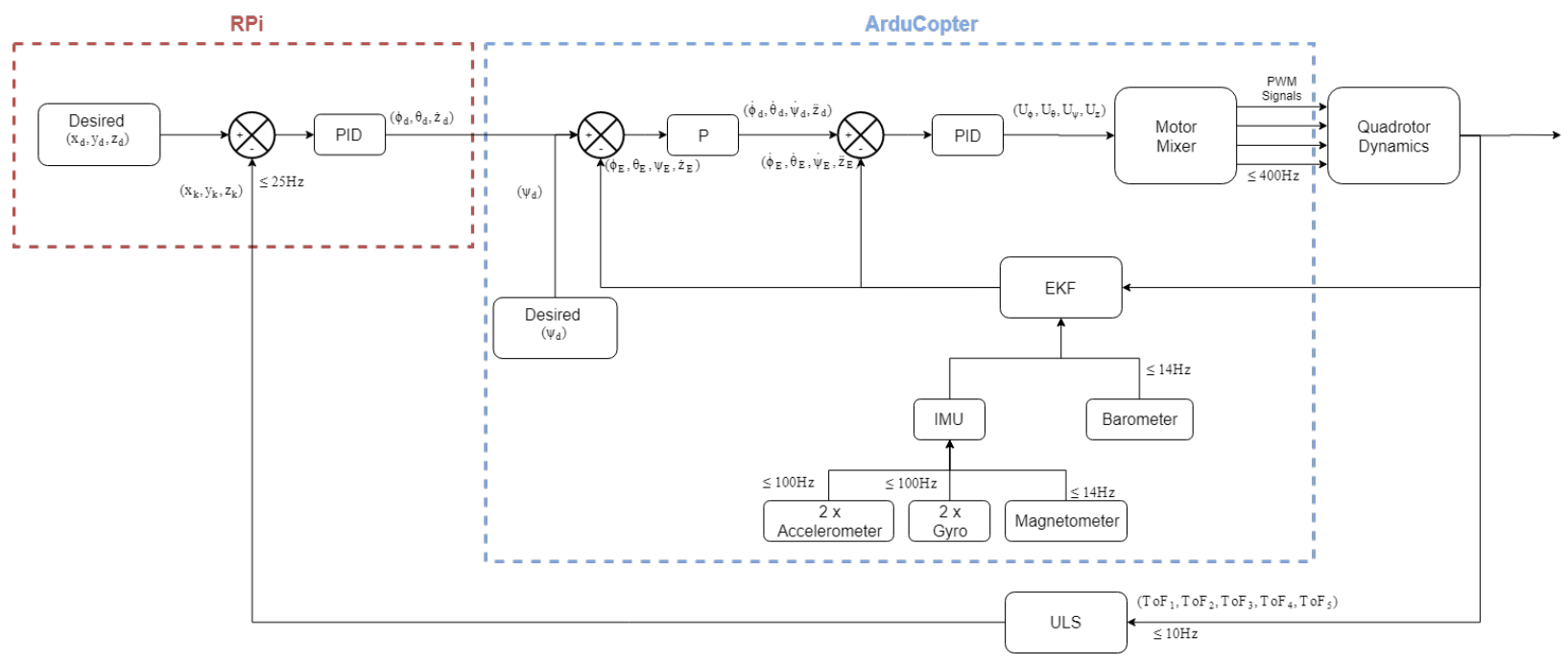

Figure 3.4: Implemented control scheme

$$
U_{\theta}=K_{P_{\dot{\theta}}} \underbrace{\left(\dot{\theta}_{d}-\dot{\theta}_{E}\right)}_{\text {rate error }}+K_{I_{\dot{\theta}}} \int_{0}^{t}\left(\dot{\theta}_{d}-\dot{\theta}_{E}\right) d t+K_{D_{\dot{\theta}}} \frac{d\left(\dot{\theta}_{d}-\dot{\theta}_{E}\right)}{d t}
$$

$$
U_{\psi}=K_{P_{\dot{\psi}}} \underbrace{\left(\dot{\psi}_{d}-\dot{\psi}_{E}\right)}_{\text {rate error }}+K_{I_{\dot{\psi}}} \int_{0}^{t}\left(\dot{\psi}_{d}-\dot{\psi}_{E}\right) d t+K_{D_{\phi}} \frac{d\left(\dot{\psi}_{d}-\dot{\psi}_{E}\right)}{d t}
$$

The gains are obtained using the auto-tune feature in ArduCopter and are provided in Table 3.4. 
Table 3.4: Attitude Stabilization Gains

\begin{tabular}{|c|c|c|c|c|c|c|c|c|c|c|c|}
\hline \multicolumn{3}{|c|}{ Angle } & \multicolumn{10}{|c|}{ Rates } \\
\hline$K_{P_{\phi}}$ & $\mathrm{K}_{P_{\theta}}$ & $\mathrm{K}_{P_{\psi}}$ & $K_{P_{\dot{\phi}}}$ & $K_{I_{\dot{\phi}}}$ & $K_{D_{\dot{\phi}}}$ & $K_{P_{\dot{\theta}}}$ & $K_{I_{\dot{\theta}}}$ & $K_{D_{\dot{\theta}}}$ & $K_{P_{\dot{\psi}}}$ & $K_{I_{\dot{\psi}}}$ & $K_{D_{\dot{\psi}}}$ \\
\hline \hline 12.3 & 11.1 & 4.87 & 0.2235 & 0.2235 & 0.00770 & 0.217 & 0.217 & 0.00705 & 1.50 & 0.150 & 0.00 \\
\hline
\end{tabular}

For altitude control, it follows the same control scheme as the attitude control but using a desired vertical velocity, $\dot{z}_{d}$ rather than a desired angle. The velocity error is calculated based off the desired velocity (Eq. 3.3) and the current velocity, $\dot{z}_{E}$, from the EKF. This produces a desired acceleration, $\ddot{z}_{d}$, which the quadrotor must operate to achieve the desired velocity. The control law is given by,

$$
\ddot{z}_{d}=K_{P_{\dot{z}}} \underbrace{\left(\dot{z}_{d}-\dot{z}_{E}\right)}_{\text {rate error }}
$$

A PID controller is implemented to stabilize the desired vertical acceleration through a motor input, $U_{Z}$. The desired acceleration, $\ddot{z}_{d}$ is produced by the previous controller and the current acceleration, $\ddot{z}_{E}$, is calculated through the ArduCopter EKF to produce the acceleration error. The control law is given as,

$$
U_{Z}=K_{P_{\ddot{z}}} \underbrace{\left(\ddot{z}_{d}-\ddot{z}_{E}\right)}_{\text {acceleration error }}+K_{I_{\ddot{z}}} \int_{0}^{t}\left(\ddot{z}_{d}-\ddot{z}_{E}\right) d t+K_{D_{z}} \frac{d\left(\ddot{z}_{d}-\ddot{z}_{E}\right)}{d t}
$$

The altitude gains are based off of the default ArduCopter values, these are provided in Table. 3.5.

Now the motor inputs for thrust, roll, pitch, and yaw $\left(U_{Z}, U_{\phi}, U_{\theta}, U_{\psi}\right)$ have all been derived. The motor mixer block combines all the independent motor inputs and converts them into individual motor pulse width modulated (PWM) signals. The 
Table 3.5: Altitude Stabilization Gains

\begin{tabular}{|l|l|l|l|}
\hline$K_{P_{\dot{Z}}}$ & $K_{P_{\ddot{z}}}$ & $K_{I_{\ddot{z}}}$ & $K_{D_{\ddot{z}}}$ \\
\hline \hline 5.000 & 0.500 & 1.000 & 0.000 \\
\hline
\end{tabular}

EKF estimates the states for feedback control. For attitude stabilization a GPS is not required because there are no feedback loops which require the position or velocity. The update rates are according to the ArduCopter source code version 3.5.5 [62]. The ULS measures the TOF's to produce the position outputs for the RPi controller.

\section{Altitude Hold}

The quadrotor is under manual control during takeoff, using altitude hold mode. After it is hovering over the beacon it is switched into Guided No GPS mode. The control scheme for the altitude hold mode is shown in Fig. 3.5. The main difference is the $\mathrm{RC}$ transmitter provides the input rather than the RPi controller. Rather than providing a desired yaw input, it provides a desired yaw rate input. ArduCopter provides the same control for this control loop as the previous besides the slight yaw difference. 


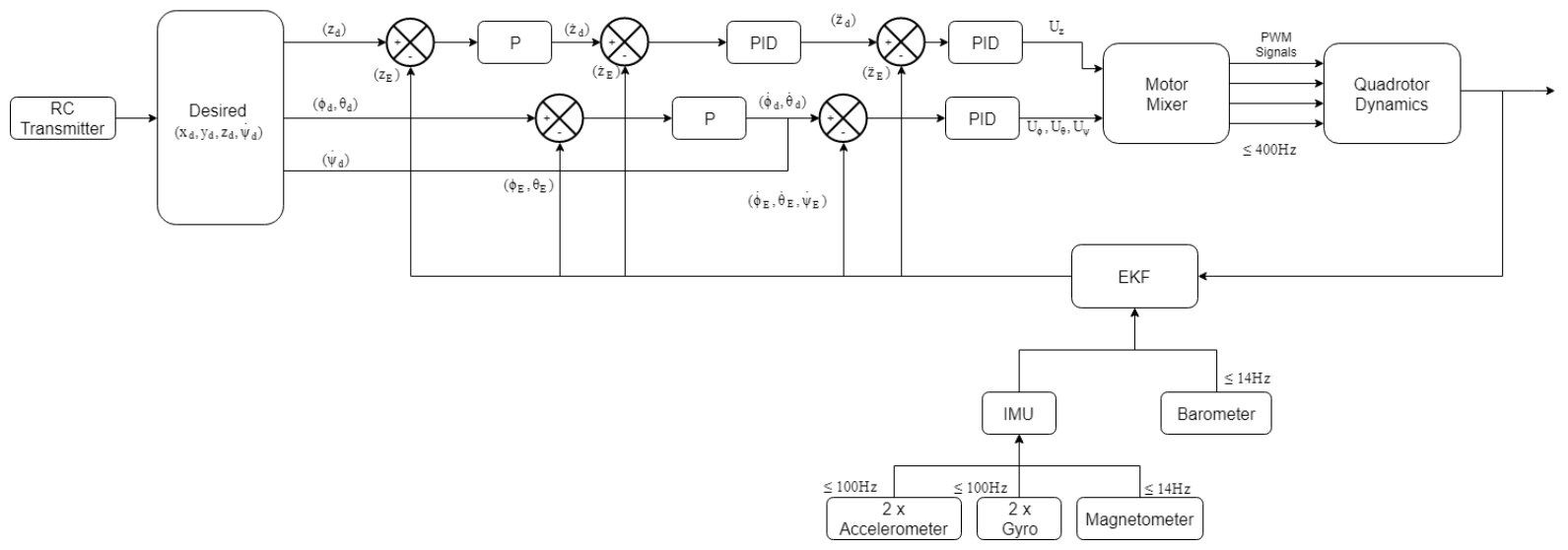

Figure 3.5: Altitude hold control block diagram 


\section{Chapter 4}

\section{Ultrasonic Localization System}

The selected localization method uses ultrasound because it is simple, cheap and can reach sub-centimeter accuracy [32]. Ultrasonic range is limited $(<5 \mathrm{~m})$ but for a landing application it is sufficient. Ultrasonic localization has successfully been used for indoor flying localization. There are no examples where the receivers are located on the drone and the beacon is capable of moving. This is likely due to the high dilution of precision (DoP) which is obtained by mounting the receivers relatively close to each other. But this provides the possibility of tracking a moving object or landing on a moving object. Currently vision systems are capable of this [27], but the ultrasonic solution requires less computer processing and works in foggy environments.

For this localization method, the ranging technique selected is ToF because it provides fast results with fewer receivers. The clocks are synchronized using a radio frequency signal propagating at the speed of light which is considered instantaneous relative to the speed of sound. This method is selected over time difference of arrival (TDoA) because TDoA requires an extra distance measurement, and the number of receivers located on the quadrotor is limited. TDoA requires a minimum of five receivers which there are, but using ToF with an additional receiver increases the

accuracy of the position estimate (as shown later with LLS). Threshold detection is selected because it provides fast results for real time application. The selected 
lateration algorithms are the analytical trilateration method and the LLS method. Since space and payload are limited on a quadrotor, the ideal setup uses only three receivers via the analytical method if the accuracy is sufficient. LLS is then tested using up to five receivers to determine the impact on accuracy.

Threshold detection is used to capture the TOF of the ultrasonic signal, such that a signal is captured if it reaches a threshold value. This method is simple and fast but leads to range and noise issues. If the threshold value is set too high the detection range is limited because of amplitude decay and interference. If the threshold is set too low the receiver is subject to noise through multipath propagation, vibrations and electromagnetic interference (EMI). The wake turbulence has been shown to decrease the received energy of the ultrasonic wave by more than a factor of 10 [65].

The following sections first introduce ToF and how it is measured on the quadrotor. Next the hardware required to measure these ToF's are discussed and this produces five ToF measurements. Then these ToF's are transferred to the software to perform lateration calculations and remove noise to provide the final position used for control, $\left(x_{k}, y_{k}, z_{k}\right)$.

\subsection{Ultrasonic Time of Flight Localization}

An ultrasonic signal can be modeled as a wave shown by Eq. (4.1).

$$
s(t)=A(t) \cdot \sin \left(2 \pi f_{c} t+\delta(t)\right)
$$

$\mathrm{A}(\mathrm{t})$ is the fluctuating amplitude which depends on the distance, the beam angle, and the rise time of the beacon. The center frequency, $f_{c}$, is chosen as $40 \mathrm{kHz}$ and the phase, $\delta(t)$, changes depending on the position of the receiver. A typical shape for the waveform produced by the beacon is shown in Fig. 4.1. The signal ramps up as the piezo-electric material begins to resonate, reaching its peak resonance depending 
on the driving voltage which is $20 \mathrm{Vpp}$ here. After 10 wavelengths the driving voltage stops and the signal begins to decay while producing waves due to the inertia of the vibrating material.

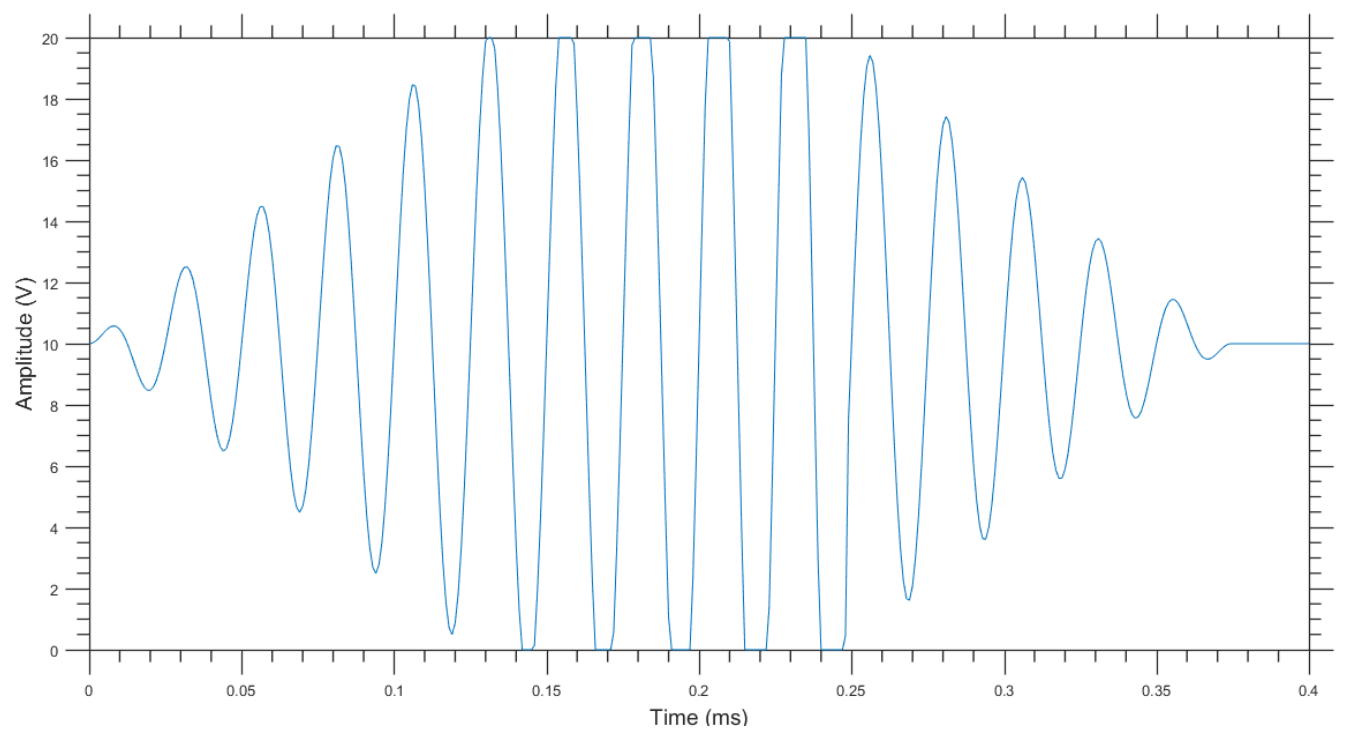

Figure 4.1: Typical ultrasonic signal produced by the beacon

The signal propagates through the air and is received by a receiver where the signal has reduced amplitude and has been phase shifted as shown in Fig. 4.2. The phase shift corresponds to a distance travelled of one metre from the beacon. The threshold voltage triggers a signal capture before the second peak, the signal capture time corresponds to the ToF.

The position of the beacon, $\boldsymbol{P}$, (see Fig. 4.3) is measured with respect to the center of the quadrotor which is used as the origin. The position of each receiving node, $\boldsymbol{R}$, relative to the origin is denoted in Fig. 4.3 and 4.4. Each receiver is uniquely identified with $\boldsymbol{R}_{1}$ in the middle, then $\boldsymbol{R}_{2}$ in the positive X and Y quadrant and the remaining receivers following a counter clockwise order. These receiver labels will be used to identify which receivers are used in a lateration algorithm. The receivers use piezo-electric transducers for ultrasonic localization mounted on arm extensions 


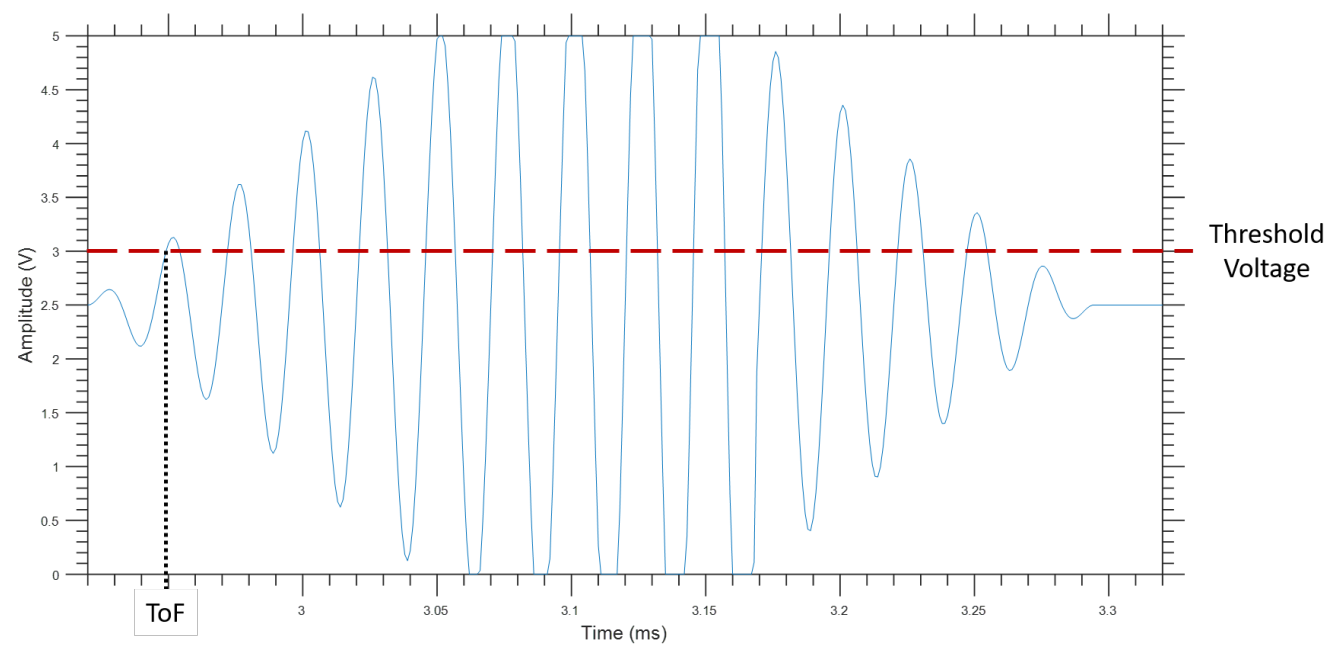

Figure 4.2: Typical ultrasonic signal received by a receiver $1 \mathrm{~m}$ away

outside of the propeller wake. The physical positions of the receivers are provided in Table 4.1. The first receiver is mounted slightly under the quadrotor due to spacial limitations.

The ToF is measured from when the ultrasonic signal is produced by the beacon to when it is received by each receiver. The distance to the beacon from each receiver can then be calculated by Eq. 4.2, where the speed of sound is given by Eq. 4.3 and $\mathrm{T}$ is the ambient air temperature.

$$
\begin{gathered}
d_{i}=a \cdot \text { TOF }_{i}, \quad i \in[1,5] \\
a=\sqrt{\gamma_{g} R_{g} T}, \quad(\mathrm{~m} / \mathrm{s})
\end{gathered}
$$

Each distance represents a spherical surface centered on the receiver as shown by Eq. 4.4. Each measurement has an error, e, due to noise and receiver positioning. The position is determined through the intersection of three or more of these spherical surfaces. The unknowns $\left(x_{p}, y_{p}, z_{p}\right)$ are all nonlinear. The positions of the receivers are known based on the geometry of the setup, $\left(x_{r i}, y_{r i}, z_{r i}\right)$. The measurement error, 


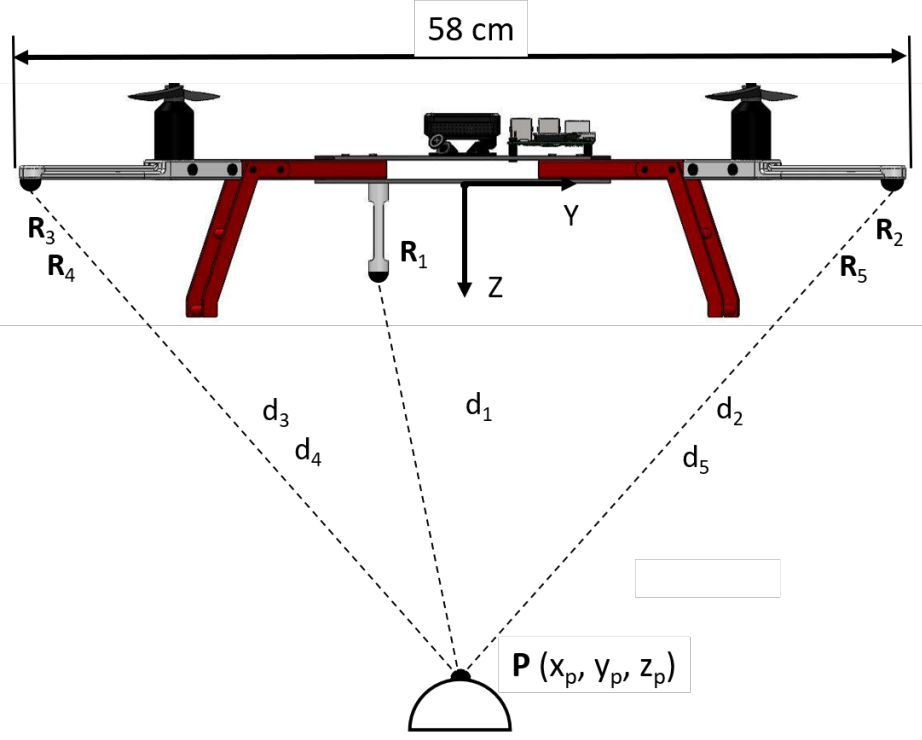

Figure 4.3: Multilateration shown on quadrotor (X into the page)

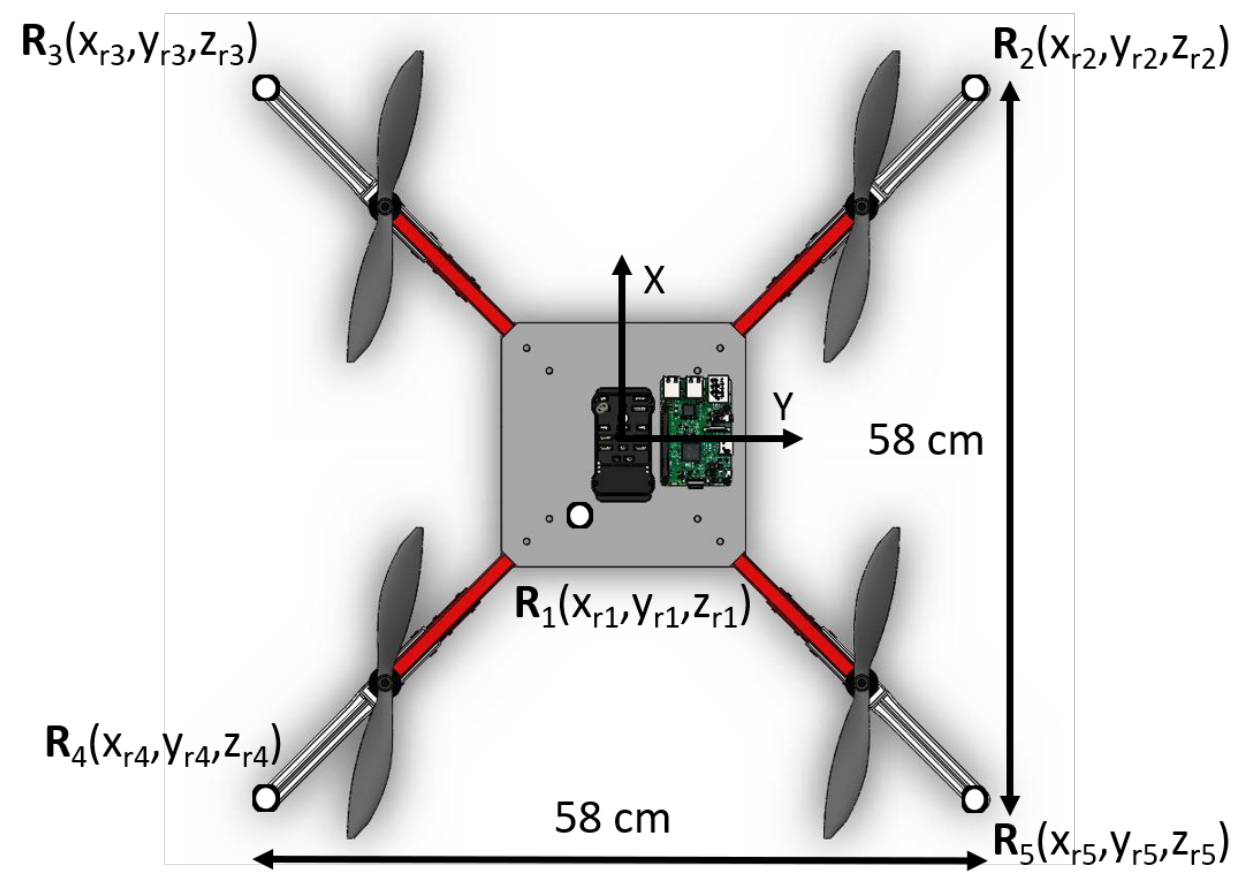

Figure 4.4: Receiver layout on quadrotor ( $\mathrm{Z}$ into the page) 
Table 4.1: Summary of the receiver positions

\begin{tabular}{|c|c|c|c|}
\hline Receiver & $x_{r}(\mathrm{~cm})$ & $y_{r}(\mathrm{~cm})$ & $z_{r}(\mathrm{~cm})$ \\
\hline \hline 1 & -5.5 & -3.5 & 6.0 \\
\hline 2 & 28.3 & 28.3 & 0.0 \\
\hline 3 & 28.3 & -28.3 & 0.0 \\
\hline 4 & -28.3 & -28.3 & 0.0 \\
\hline 5 & -28.3 & 28.3 & 0.0 \\
\hline
\end{tabular}

$e_{i}$, is unknown but is minimized using LLS or assumed to be zero for the analytical method. The distance measurements, $d_{i}$, are calculated through the measured ToF's every time a position is requested.

$$
\left(x_{p}-x_{r i}\right)^{2}+\left(y_{p}-y_{r i}\right)^{2}+\left(z_{p}-z_{r i}\right)^{2}+e_{i}^{2}=d_{i}^{2}, \quad i \in[1,5]
$$

\subsection{Hardware}

The ULS consists of a beacon located on the ground and five receivers located on the quadrotor. The infomation from these receivers is combined by a master controller on a PSoC located on the quadrotor. The PSoC consists of two components, digital hardware, and the main control loop. The components in the main control loop will be discussed in the next section. The system overview is shown in Fig. 4.5. The localization process is started by the main control loop through a signal request. The digital hardware forwards this request to the beacon through XBee wireless 
communication and notes the time of this request. The beacon contains an Arduino Nano which is a micro controller that handles requests from the PSoC and produces ultrasonic signals. Once it receives a request it produces a $40 \mathrm{kHz}$ square wave which is then amplified to $20 \mathrm{Vpp}$. This signal drives a piezo-electric speaker which produces an ultrasonic wave through the inverse piezo-electric effect. This signal is received by five piezo-electric transducers mounted on the quadrotor. The signal is converted to an electrical signal through the direct piezo-electric effect. It is then amplified and filtered to remove unwanted noise. A comparator is used for threshold detection and if the signal is above a certain amplitude it converts it to a digital signal. Once the digital signals are captured the clock which was started at the signal request is stopped and the ToF is calculated. As there are five receivers on the quadrotor this generates five ToF values which are sent to the main control loop for calculations. The final position calculated by the main control loop is represented by $\left(x_{k}, y, z_{k}\right)$ and is sent to the RPi for control.

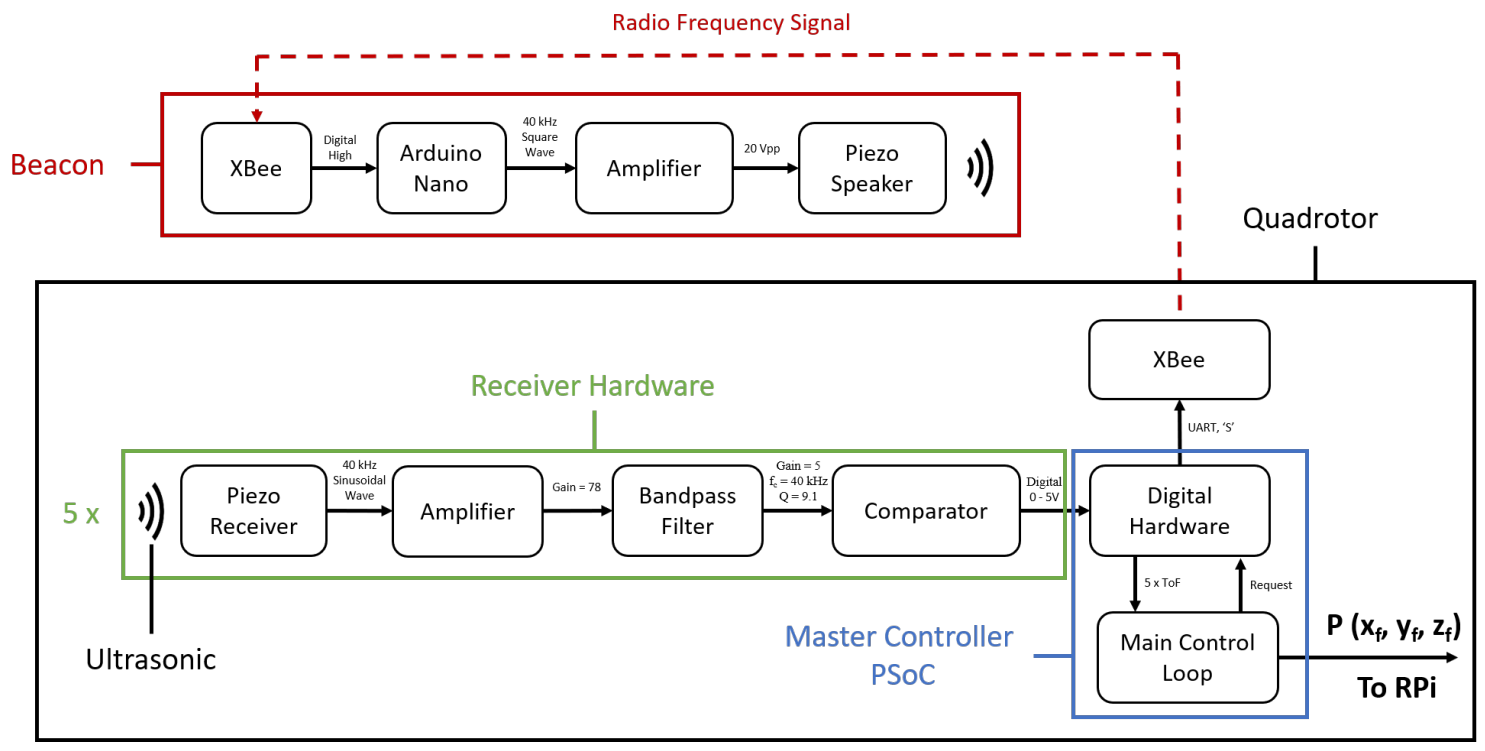

Figure 4.5: Ultrasonic Localization System block diagram

The hardware section determines the ToF for the five receivers which it sends 
to the main control loop. The next subsections introduce piezo electric transducers, these are used to transmit and receive the ultrasonic signals. Next the hardware required to generate an ultrasonic signal in the beacon is discussed follwed by the receiver hardware which turns the received ultrasonic signal into a digital signal. Finally the digital hardware which keeps track of the time and calculates the ToF is described. Based on the selected hardware and configuration the theoretical distance can then be calculated.

\subsubsection{Piezo Electric Transducer}

Piezo-electricity refers to the process of converting mechanical energy into electrical energy or electrical energy into mechanical energy. Piezo-electric materials make this possible through their unique unsymmetrical unit cell. When these cells are subject to a mechanical stress they deform and obtain an electrical potential. Typically, ultrasonic transducers use lead zirconate titanate. Applying mechanical energy to the piezo-electric material is referred to as direct piezoelectric effect. The opposite is referred to as inverse piezoelectric effect [66].

A schematic of an open type piezo-electric transducer by Murata is shown in Fig. 4.6. The piezo-electric ceramic is sandwiched between two metal plates and each plate is connected to an electric lead. The vibrating section consists of the horn, metal plates and the piezoelectric ceramic. The bottom metal plate is fixed elastically to the base. For the direct piezoelectric effect, the incoming ultrasonic wave causes the vibrating section to resonate at the design frequency. This resonance causes the piezoelectric ceramic to flex and generates a voltage at the same frequency. For the inverse effect a voltage is supplied to the lead terminals at the design frequency. This causes the piezoelectric ceramic to vibrate, which causes the horn to resonate ultrasonic signals.

The MA40S4S and the MA40S4R are used for the transmitting and receiving 


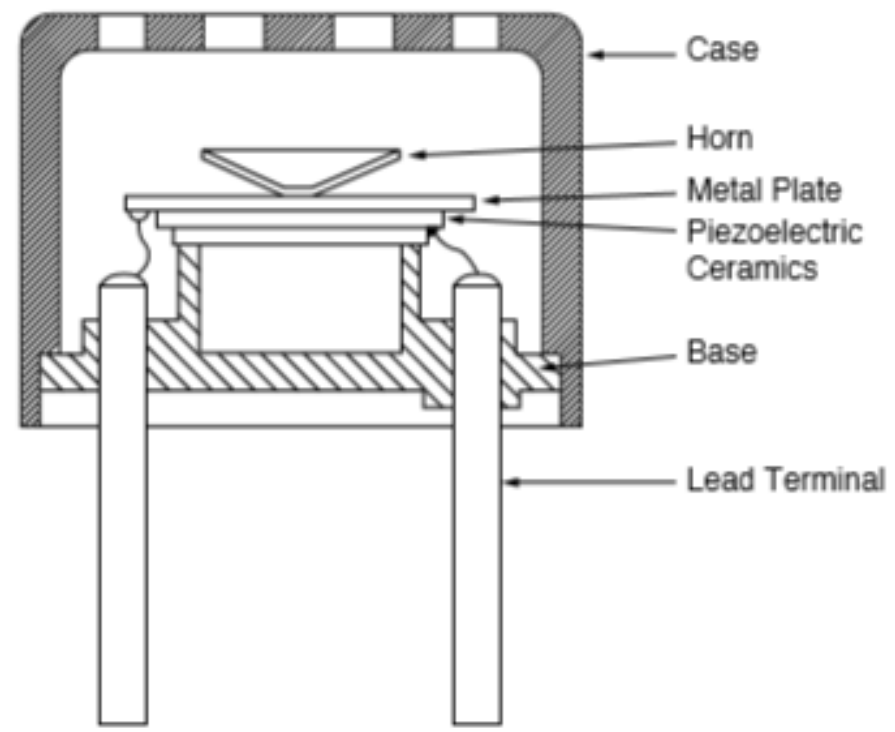

Figure 4.6: Ultrasonic transducer schematic [2]

sections respectively. They are selected because they are inexpensive transducers which operate with a high SPL and receive at a high sensitivity.

\subsubsection{Beacon}

The beacon consists of an XBee wireless receiver, Arduino Nano, amplifier and piezoelectric speaker. The XBee operates in unicast mode to reduce delay as it only needs to be paired with an XBee on the quadrotor. The XBee is connected to a digital port on the Arduino Nano rather than the serial port to decrease the dropped signals. An analysis is performed to determine that $99.4 \%$ of the signals are successfully transmitted and received by the XBee receivers. The Arduino Nano produces a 5 Vpp ultrasonic square wave which is sent to an amplifier.

The length of the ultrasonic signal is altered to assess the impact this has on the accuracy of the resulting position measurement. The period of a $40 \mathrm{kHz}$ ultrasonic 
signal is $25 \mu$ s and thus in Fig. 4.7 every two periods corresponds to a signal time of $50 \mathrm{~ms}$. Figure 4.7 shows that when the number of periods set to 1 or 5 periods ( 25 or $125 \mathrm{~ms}$ ) the number of successful measurements is low. This is because the amplitude of the wave is not yet high enough to trigger a signal capture using the threshold method. As the number of periods is increased to 10 and 15 periods (250 and $375 \mathrm{~ms}$ ) a maximum successful position rate of $72 \%$ is observed. The beacon and quadrotor are fixed with the beacon, located $177 \mathrm{~cm}$ beneath and $100 \mathrm{~cm}$ next to the quadrotor. As data is calculated over $60 \mathrm{~s}$, comparing the successful position measurements to the known location yields a standard deviation of $5.3 \mathrm{~cm}$. Increasing the length to 15 periods (375 ms) does not increase the success rate and actually increases the standard deviation and so a nominal value of 10 periods is selected.

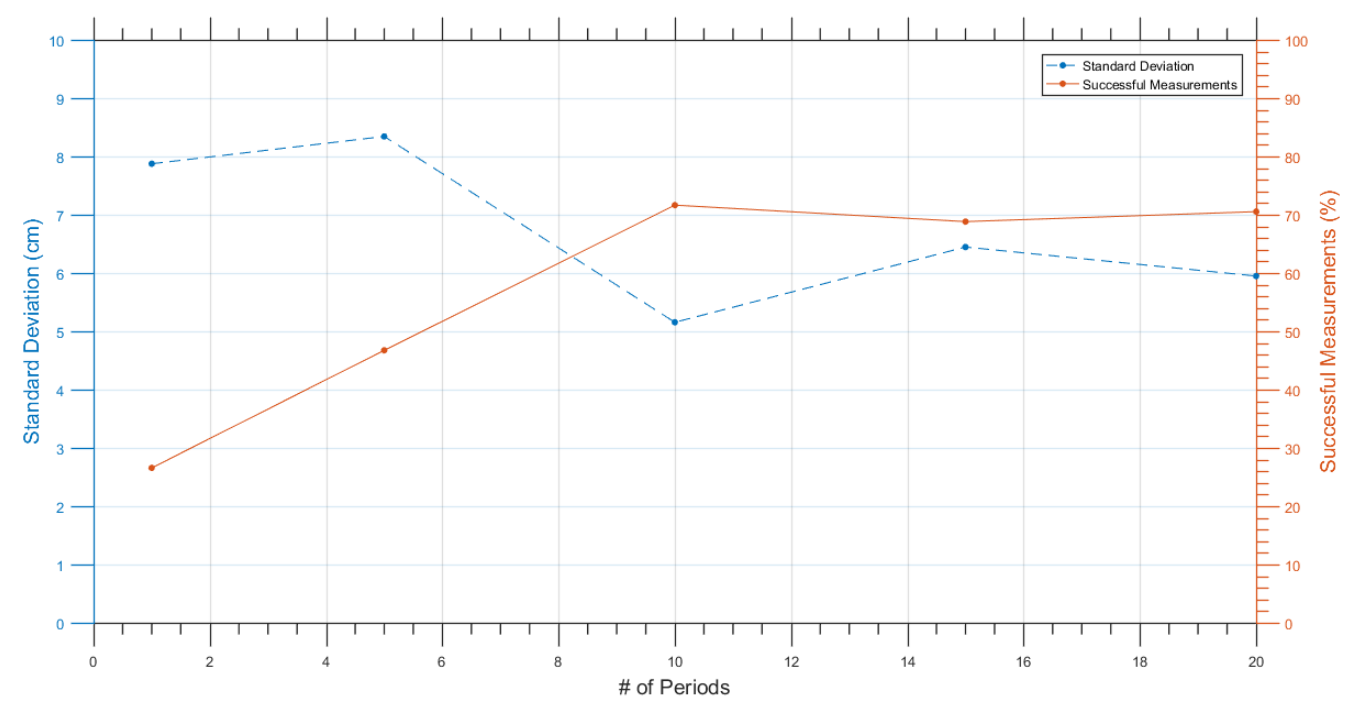

Figure 4.7: Transmission length accuracy $\mathrm{P}(100,0,177)$

The signal amplifier circuit is designed on a printed circuit board (PCB) shown in Fig. 4.8. A $22 \mathrm{~V}$ lithium polymer battery is used to power the amplifier. A non-inverting amplifier with a gain of four is used to increase the signal voltage to $20 \mathrm{~V}$. This is the maximum allowable voltage for the piezo electric transducer [67]. An MCP6022 is selected as the op amp because it has stable operation in the $40 \mathrm{kHz}$ 
frequency range and can be powered by a $22 \mathrm{~V}$ single voltage and it is inexpensive. The output of the non-inverting amplifier is connected to five voltage buffer circuits (only one is shown in Fig. 4.8). Each voltage buffer can be connected to an ultrasonic transducer shown as JP1. The five voltage buffers allow each speaker to be isolated, preventing electrical interference.

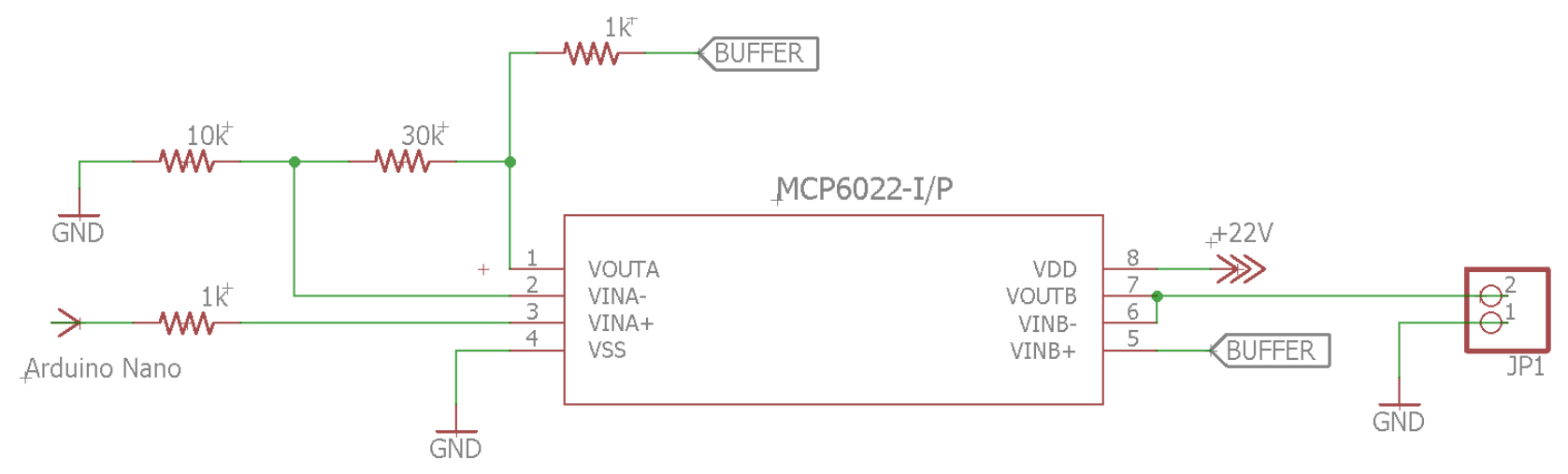

Figure 4.8: Beacon signal amplifier

There are five available channels which a speaker can connect to, these speakers can be mounted on a hemisphere to provide full hemispherical coverage as shown in Fig. 4.9. An analysis determined that the accuracy decreases using five speakers versus one speaker mounted vertically. The decrease in accuracy is attributed to wave interference when multiple speakers are producing an ultrasonic wave simultaneously. To remove this effect only the single transducer mounted vertically is used for testing.

\subsubsection{Receiver Hardware}

There are five piezo electric transducers mounted on the arms and center of the quadrotor. Having the receivers mounted on the drone makes the captured signal susceptible to noise through electromagnetic interference (EMI) from the motors and any mechanical vibrations. To mitigate these issues a shielded wire is used to carry the signal from the transducer to the amplifier and a bandpass filter is used to reduce 


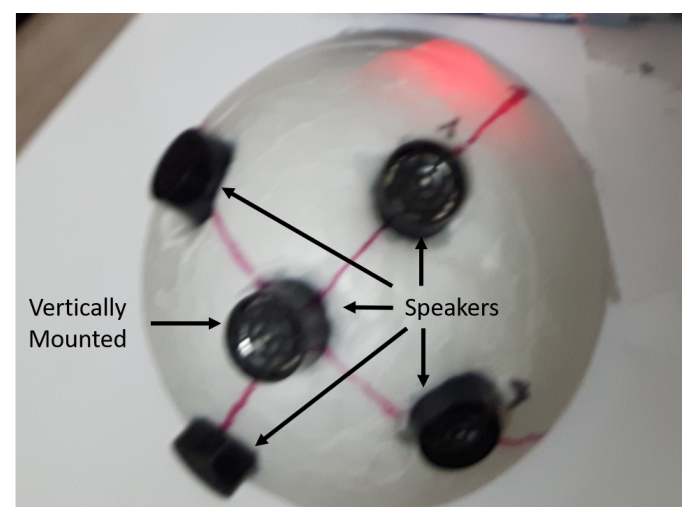

Figure 4.9: Beacon top view

electrical noise away from $40 \mathrm{kHz}$. The shielded wire is grounded at one end which forces the electrical potential of the shield to be $0 \mathrm{~V}$. This eliminates the effect of any surrounding electrical field on the captured signal.

The circuit design for a single channel is shown in Fig. 4.11. The piezo-electric transducer connects to a two-conductor shielded wire (Belden 8451 [68]) which connects to channel 0 (CHOI) as shown in Fig. 4.10. The bottom port, channel 3, is connected to the shield and grounded to reduce EMI. As shown on the figure the receiver sensitivity regulates how sensitive and noisy the signal is, choosing a higher resistance leads to a more sensitive and noisier signal. A moderate value of $3.9 \mathrm{k} \Omega$ is chosen according to the sensitivity values given in Fig. 4.12 from the Murata ultrasonic application manual [2]. The signal is amplified using a differential amplifier with a gain of 78 . The signals are treated as a differential voltage to remove any common mode noise that may have been experienced in the wire. MCP6022 is selected as the op amp as it has good performance in the $40 \mathrm{kHz}$ frequency range and it can be powered from a $5 \mathrm{~V}$ supply.

The next three circuits are a voltage divider, voltage buffer, and a bandpass filter. The voltage divider (located at the bottom Fig. 4.11) provides a bias voltage of 2.5 $\mathrm{V}$, this allows the signal to fluctuate $\pm 2.5 \mathrm{~V}$. The second circuit is a voltage buffer 


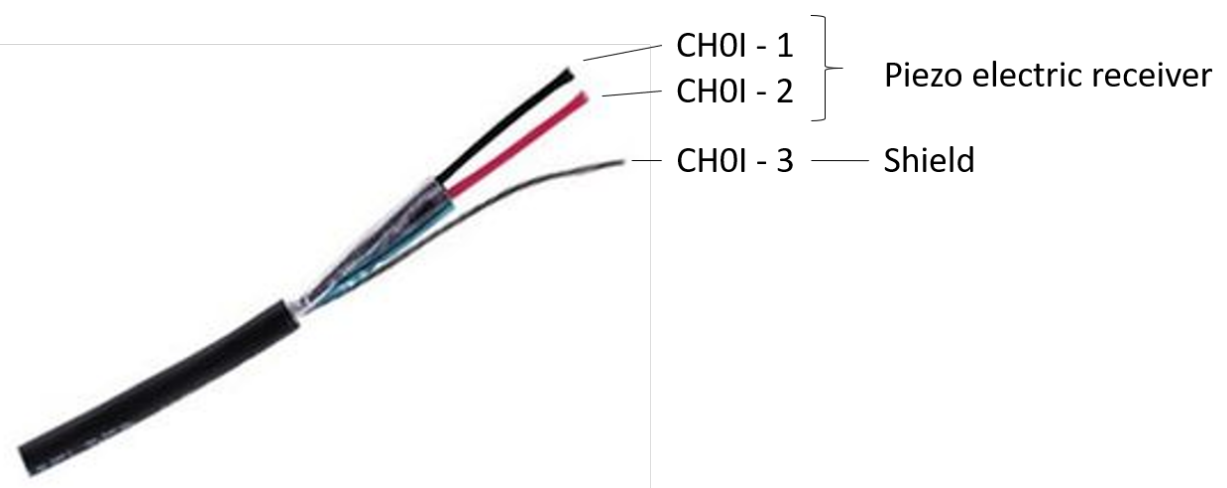

Figure 4.10: Wire connections

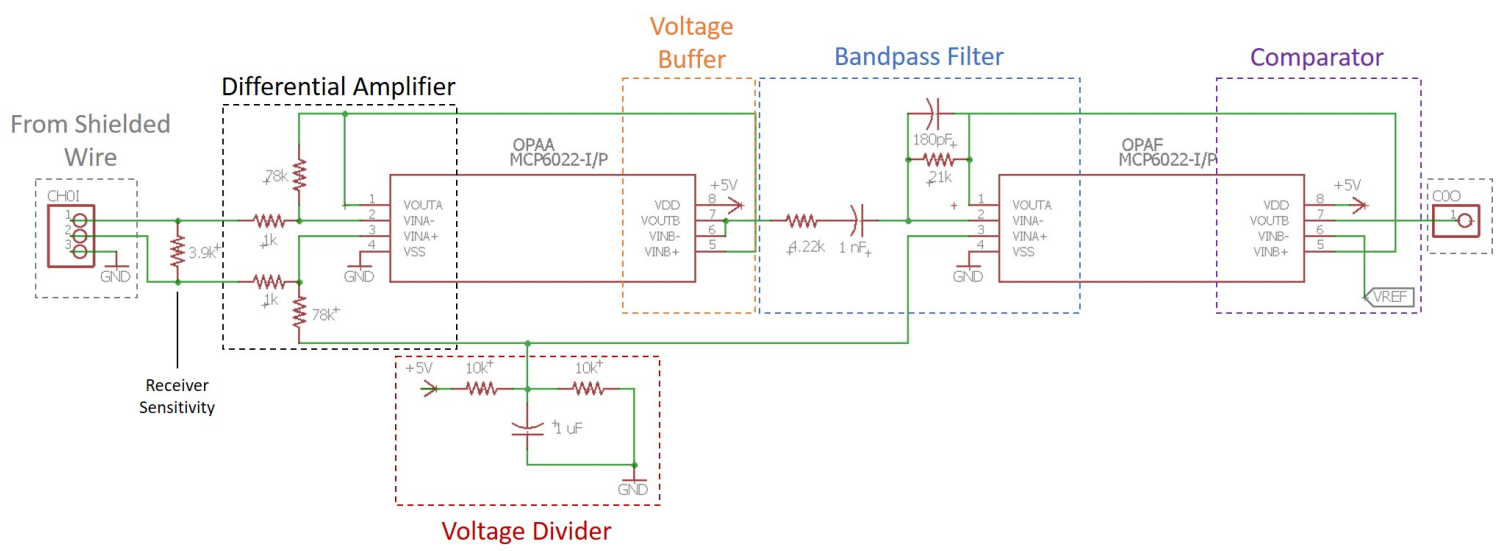

Figure 4.11: Receiver PCB

which is used to isolate the amplifier circuit from the following filter circuit. The third circuit is an inverting band pass filter with parameters as listed in Table 4.2. A narrow bandwidth is selected matching to the relatively narrow peak of the receiver sensitivity shown in Fig. 4.12. A gain of 5 increases the total circuit gain to 390 which improves detection range.

The final circuit is a comparator where the signal voltage is compared to a reference voltage, vref (adjusted using a potentiometer). If the signal voltage is greater than the reference voltage a digital high signal is sent to the PSoC. Signal noise is measured when running the motor at full throttle with a propeller. The input of the comparator 


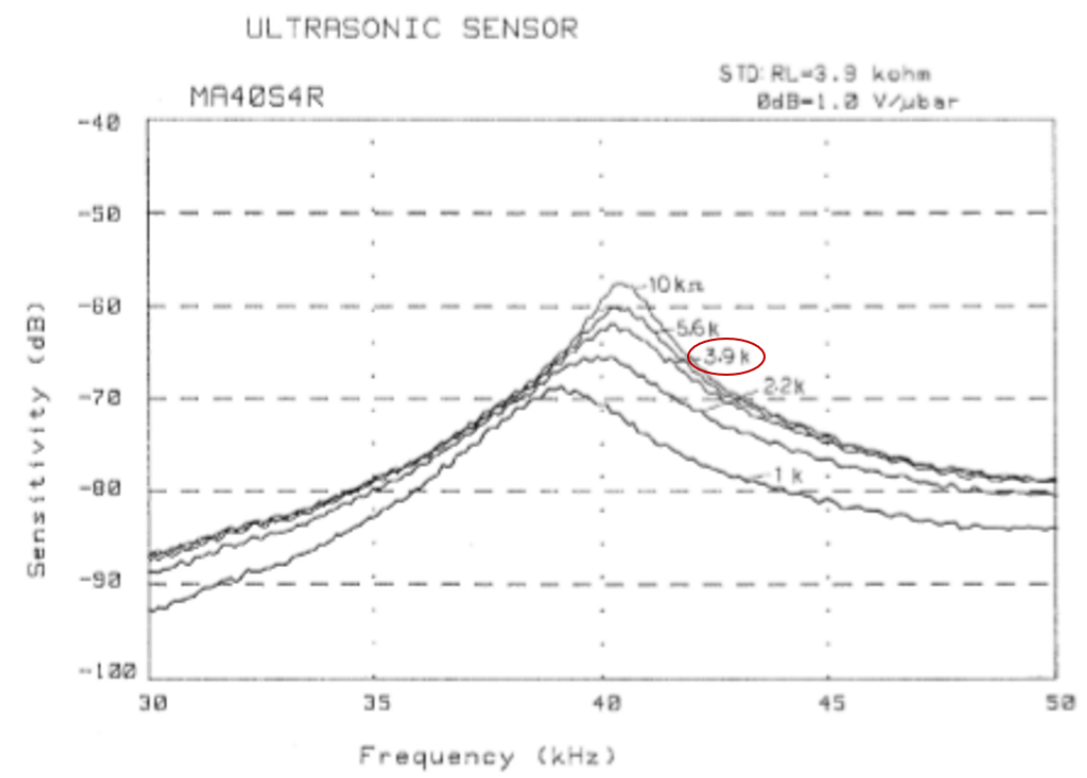

Figure 4.12: Receiver sensitivity [2]

is connected to an oscilloscope and the peaks of the noise fluctuate between $2.26 \mathrm{~V}$ and $2.66 \mathrm{~V}$. Therefore, the reference voltage is set to $3.0 \mathrm{~V}$ while the threshold detection voltage is set at $0.5 \mathrm{~V}$. 
Table 4.2: Band pass Filter Parameters

\begin{tabular}{|c|c|c|}
\hline Parameter & Value & Units \\
\hline \hline Center Frequency $\left(F_{c}\right)$ & 40 & $\mathrm{kHz}$ \\
\hline Bandwidth & 4.4 & $\mathrm{kHz}$ \\
\hline Quality Factor $(Q)$ & 9.1 & $\mathrm{~N} / \mathrm{A}$ \\
\hline Gain & 5.0 & $\mathrm{~N} / \mathrm{A}$ \\
\hline
\end{tabular}

\subsubsection{PSoC Digital Hardware}

The PSoC performs the ToF calculation using digital signal processing. The overall digital layout to measure the ToF of the signal is shown in Fig. 4.13. There are two processes this handles, it must start the ToF timer when a signal is requested and it must stop the timer once a digital signal is received from the comparator for each channel. Each channel represents the signal produced by a receiver. Each block is in reset mode when they are not expecting an ultrasonic signal (signal decay time). They are taken out of reset mode when an ultrasonic signal is requested.

\section{Start}

The process begins with the XBee UART block. The letter ' $\mathrm{S}$ ' is transmitted to the XBee on TARA 3 through UART protocol. The XBee located on TARA 3 then transmits a RF signal to the XBee receiver in the beacon to produce an ultrasonic signal. Once the UART signal has been transmitted, an interrupt signal is produced by tx_interrupt which is connected to trigger on the TOF_counter block.

The trigger starts a 16-bit timer located in the TOF_counter block. The timer 


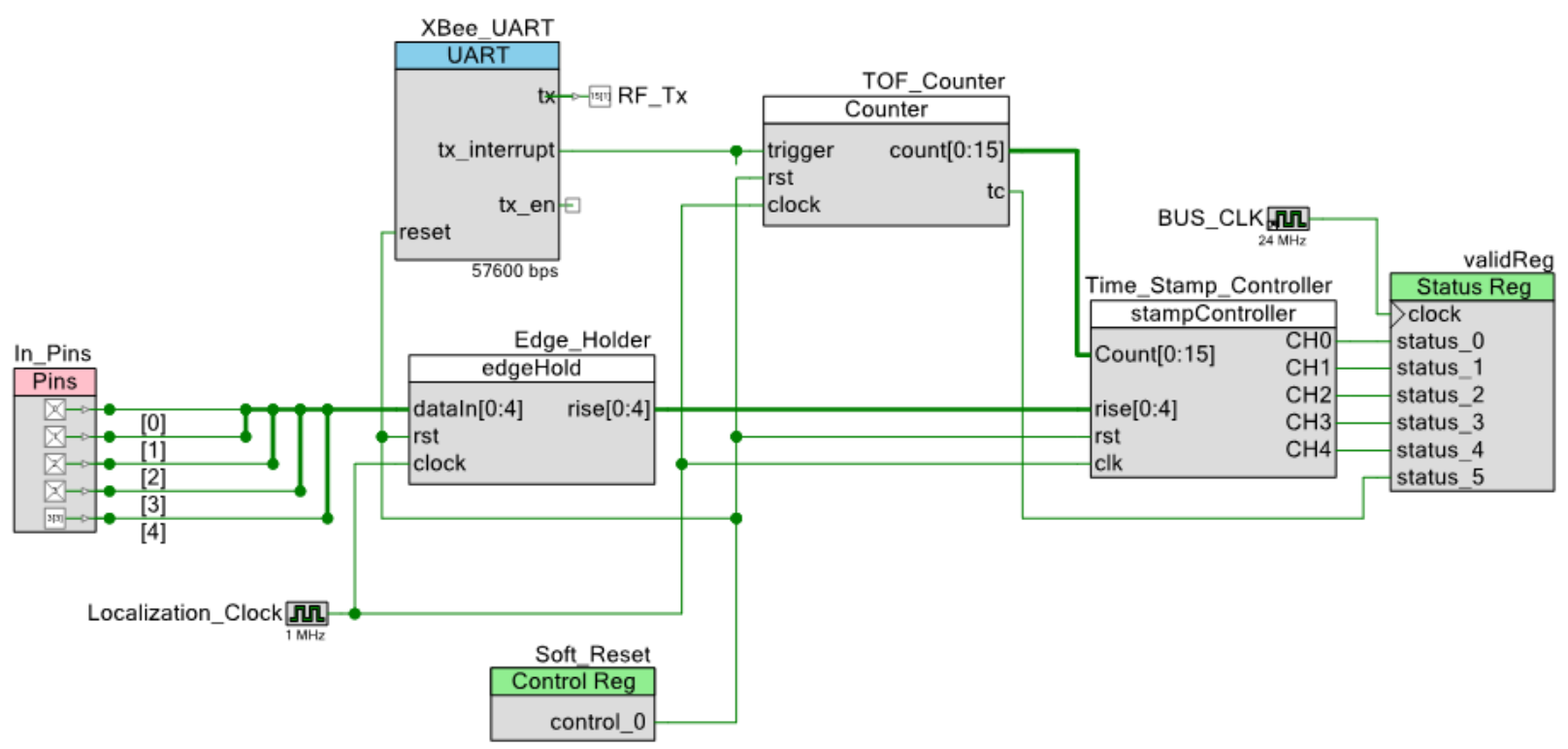

Figure 4.13: PSoC ToF digital logic

measures the ToF of the ultrasonic signal with a resolution governed by Localization_Clock. A clock speed of $1 \mathrm{MHz}$ is selected to provide a balance between resolution and maximum detection distance. This speed provides a resolution $1 \mu s$ or 0.34 mm using the speed of sound which is less than $4 \%$ of the wavelength. This resolution ensures it is not a limiting factor since threshold detection does not account for the phase of the wave. Using a 16-bit timer, the maximum ToF is $65.5 \mathrm{~ms}$ which corresponds to a detection distance of $22 \mathrm{~m}$. This range exceeds the maximum detection range for an ultrasonic system and provides time for echoes to dissipate. It produces the 16 bit count, through the output count where (0:15) represents the 16 bits of data. If the 16-bit timer finishes it sends a signal through the output, tc, to status_5 in the status register. The status register block communicates to the main control loop by providing a channel's status. Status_5 indicates in the main control loop that the timer has completed. The status register is checked using a faster clock, $24 \mathrm{MHz}$, to minimize delay.

There is a constant time delay between when the timer starts and the transmission 
of the ultrasonic signal. The time delay accounts for the beacon and receiver hardware delay. The ToF is calculated by Eq. 4.5,

$$
\text { ToF }_{i}=\text { Timer }_{i} / \text { Clock }- \text { Delay }, \quad i \in[0,4]
$$

Where Timer is the 16-bit timer count for the channel, clock is the $1 \mathrm{MHz}$ clock speed and Delay is the constant hardware delay. The delay is experimentally determined to be 6.4 ms by measuring the ToF using a known position.

\section{Stop}

The digital signal for all five channels from the comparators are shown on the left of Fig. 4.13, the lines connect to create a bus. This software starts the channel numbering from zero rather than one when describing the receivers. In this software the receiver numbers are decreased by one, that is $R_{1}=$ channel 0 . The goal is to capture the time of the first rising edge for each channel. This corresponds to the first ultrasonic wave to reach the receiver with an amplitude larger than the threshold. The bus connects to the edge holder, this block is designed to detect the first rising edge and hold the signal high while the ToF is processed. The output, rise, connects to the time stamp controller block to process the ToF for each channel and transfer the data to the main control loop for processing. The time stamp controller block sends a validation signal for each channel to the status register once a ToF has been successfully captured. The status register communicates to the main control loop when each ToF has been captured.

The edge holder block is designed to hold the signal high once a rising edge is detected, the block is shown in Fig. 4.14. It receives dataln which is a bus that contains the five different digital channels shown by (0:4). It consists of an SR flip flop and an OR block. The block starts in reset mode which ensures the output is low. It is taken out of reset mode when an ultrasonic signal is requested. Once a 
rising edge is detected from the digital data line in the output flips and stays high even if the data line goes low. This ensures the output stays high until the block is reset. The digital high signal means that an ultrasonic signal has arrived, it is sent to the Time stamp controller block through the rise output to stop the clock and calculate the ToF.

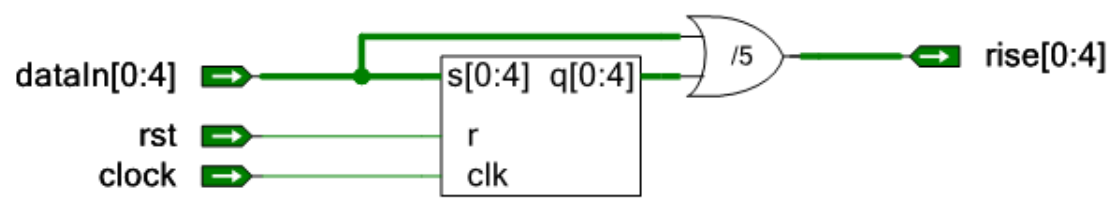

Figure 4.14: Edge holder block

The time stamp controller block (Fig. 4.15) contains the individual channel stamp blocks. It takes in the 16-bit count through the input Count and the signal from the edge holder block through the input rise. This block divides the digital bus into separate channels such that each ToF can be handled independently. It outputs a signal for each block $(\mathrm{CH} 0, \mathrm{CH} 1, \mathrm{CH} 2, \mathrm{CH} 3, \mathrm{CH} 4)$ which indicates a ToF has been captured for that channel.

A channel stamp block is shown in Fig. 4.16. The inputs are the 16 bit count (through count) and the individual channel signal (through rise). Once the reset signal is off, a pulse can be triggered. If the rise signal is high, it triggers the D flip flop. This triggers a one clock length pulse on the rising edge of the signal. The clock is divided into the least significant bit (LSB) and most significant bit (MSB) because of how the FIFO block works. The first in first out (FIFO) block receives this pulse, and captures the current time, this corresponds to the Timer value in Eq. 4.5. The DMA block transfers the time to the main control loop, where the ToF is actually calculated using Eq. 4.5. Once the transfer completes, a valid signal is produced. The FIFO block allows the time to be captured the instant a risen pulse is received. 


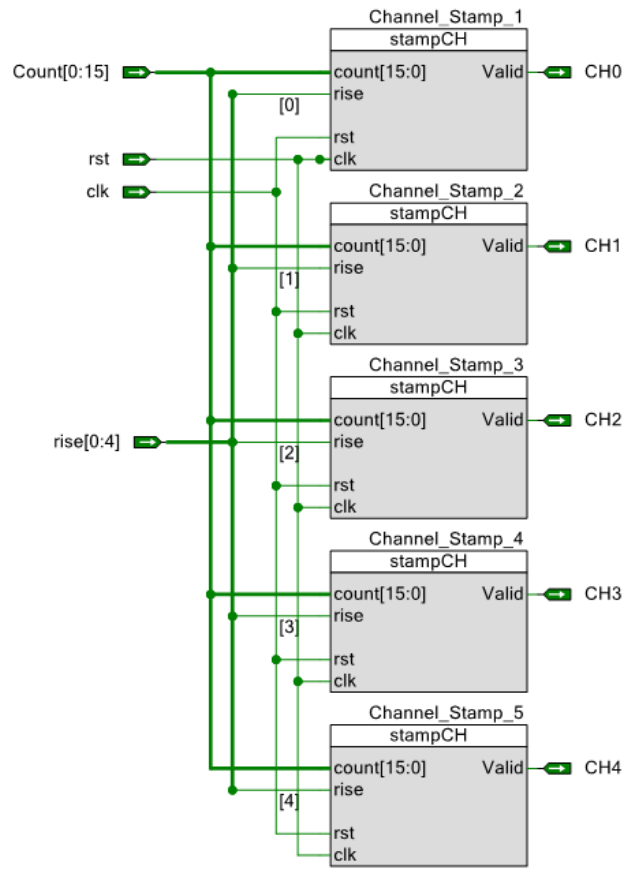

Figure 4.15: Time Stamp Controller block

If multiple channels are received at the same time, DMA can only handle one at a time. This would create a delay in the time capture without the FIFO block. DMA is typically used to transfer data in the background which allows the main control loop to continue to perform its functions without a delay.

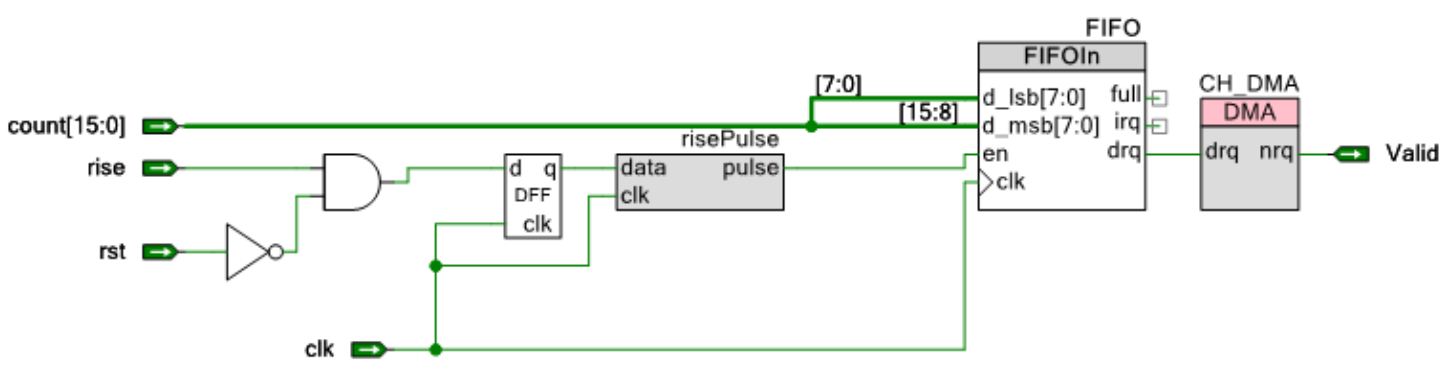

Figure 4.16: Stamp channel block 


\subsubsection{Theoretical Detection Distance}

The theoretical detectable distance using a threshold voltage of $0.5 \mathrm{~V}$ is calculated as follows. An amplifier gain of 390 (Fig. 4.11) reduces the required received signal to $1.28 \mathrm{mV}$. This is converted into a sound pressure level using the sensitivity of the receiver. The sensitivity frequency spectra for the receiver using a reference $40 \mathrm{kHz}$ wave is shown in Fig. 4.18. The peak sensitivity, $S_{d b}$, is approximately $-63 \mathrm{~dB}$ at a frequency just above $40 \mathrm{kHz}$. Along with a reference sensitivity, $S_{0}$ (given as 10V/Pa $[2])$, which using,

$$
S_{d B}=20 \log \left(S / S_{0}\right)
$$

yields a sensitivity, $S$, of $7 \mathrm{mV} / \mathrm{Pa}$. The required pressure level, $P$, can be found using,

$$
\text { Voltage }=S * P
$$

and is calculated as $0.18 \mathrm{~Pa}$ using the received signal strength of $1.28 \mathrm{mV}$. This is then converted to decibels using,

$$
S P L=20 \log \left(P / P_{0}\right)
$$

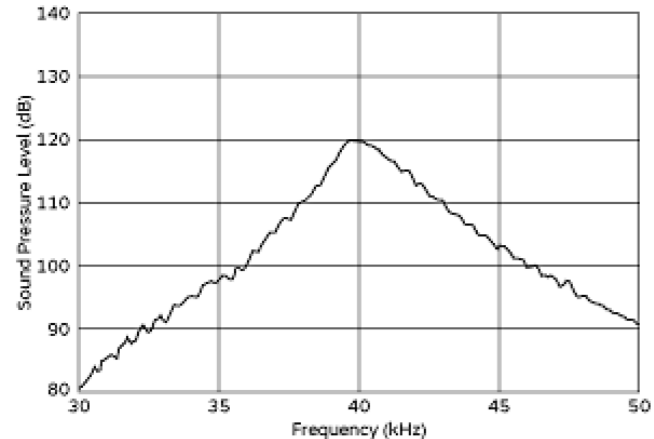

Figure 4.17: Beacon SPL [2]

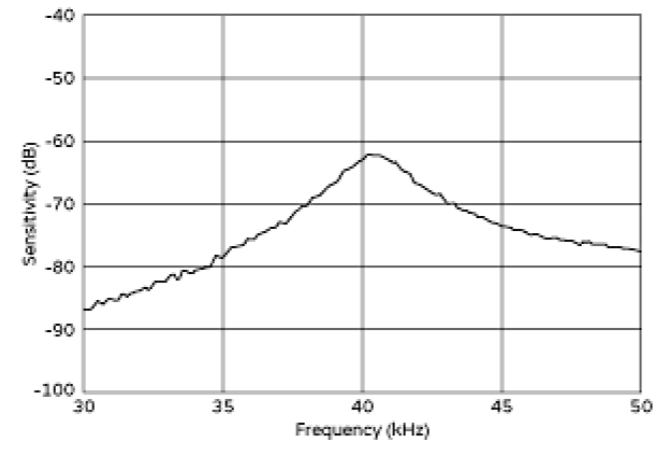

Figure 4.18: Receiver sensitivity [2] 
yielding a received SPL of $79 \mathrm{~dB}$. The frequency spectra of the transmitted sound pressure using a 10 Vrms wave is shown in Fig. 4.17. The peak transmitted frequency is at a SPL of $120 \mathrm{~dB}$ at a frequency just below $40 \mathrm{kHz}$. This allows for $41 \mathrm{~dB}$ of attenuation. The transmitted SPL decreases according to the $1 / d^{2}$ law as shown in Fig. 4.19. Neglecting atmospheric effects and directivity $(\gamma=0)$ the maximum theoretical detection distance is 8.8 metres as shown by the dashed line. In practice this range is not achieved because of atmospheric effects and noise.

If a half angle of $\gamma=45^{\circ}$ is considered between the transmitter and the receiver the distance decreases. The beam pattern for the speaker is shown in Fig. 4.20. At approximately $45^{\circ}$ there is a $5 \mathrm{~dB}$ attenuation, beyond this the attenuation quickly increases. The beam pattern for the receiver is shown in Fig. 4.21 , at a $45^{\circ}$ angle there is an approximately an $8 \mathrm{~dB}$ attenuation, beyond this the attenuation greatly increases. The maximum theoretical distance at this beam angle is $2.4 \mathrm{~m}$, shown as a dotted line in Fig. 4.19. Beyond a half angle of $45^{\circ}$ the attenuation quickly increases, decreasing the maximum detection distance.

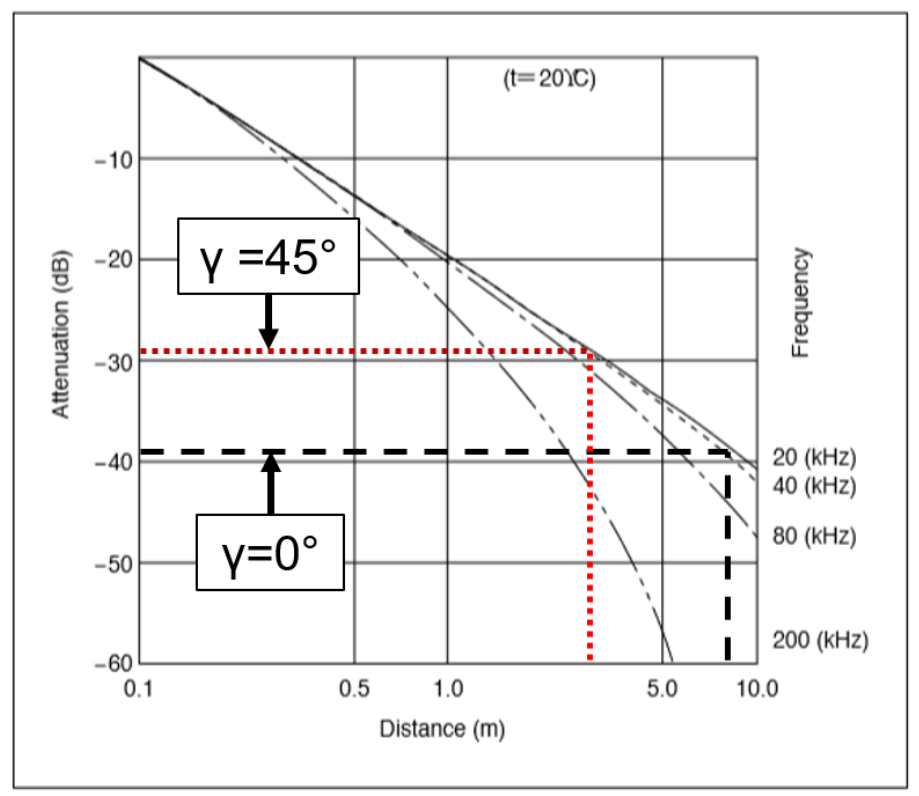

Figure 4.19: Attenuation of the ultrasonic signal [2] 


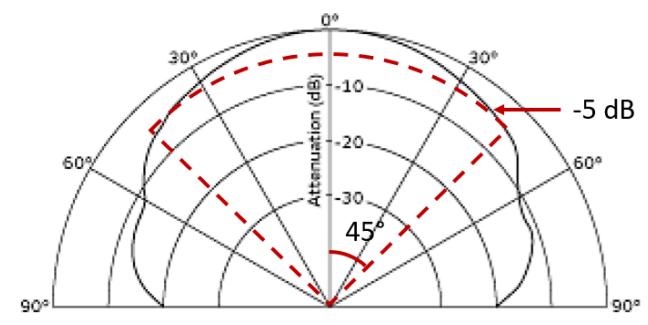

Figure 4.20: Beacon beam pattern [2]

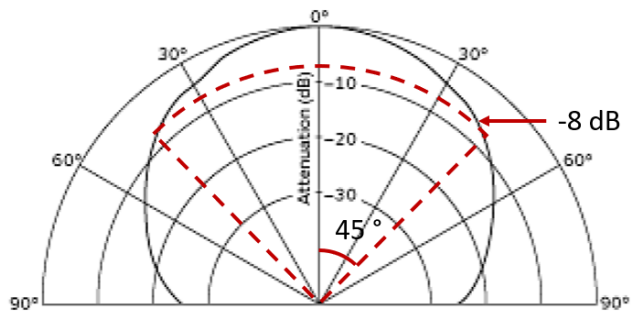

Figure 4.21: Sensitivity beam pattern

\subsection{Main Control Loop}

The main control loop is responsible for localization calculations. It receives the ToF measurements from the digital hardware on the PSoC. This section breaks down as follows, first there are two lateration techniques discussed, the analytical trilateration and LLS. The DoP is calculated to assess the configuration of the system, this is not calculated in the main control loop but is addressed here as it follows the lateration methods. To reduce noise in the position data, a Kalman filter is applied. All of these components are combined in the main control loop through an algorithm depending on the lateration technique.

\subsubsection{Analytical Method}

The analytical expression requires three receivers to be oriented on an XY plane. Any three receivers can define a local plane which is centered on a receiver, this will be known as the analytical frame. Using the analytical local frame the position of the beacon can be calculated through trilateration. This can be translated and rotated to the local coordinate frame centered on the quadrotor, which will be known as the quadrotor local frame.

The' notation means the position is in the analytical local frame as shown in Figs. 4.22 and 4.23. The axis with no' represents the quadrotor local frame. Receivers 


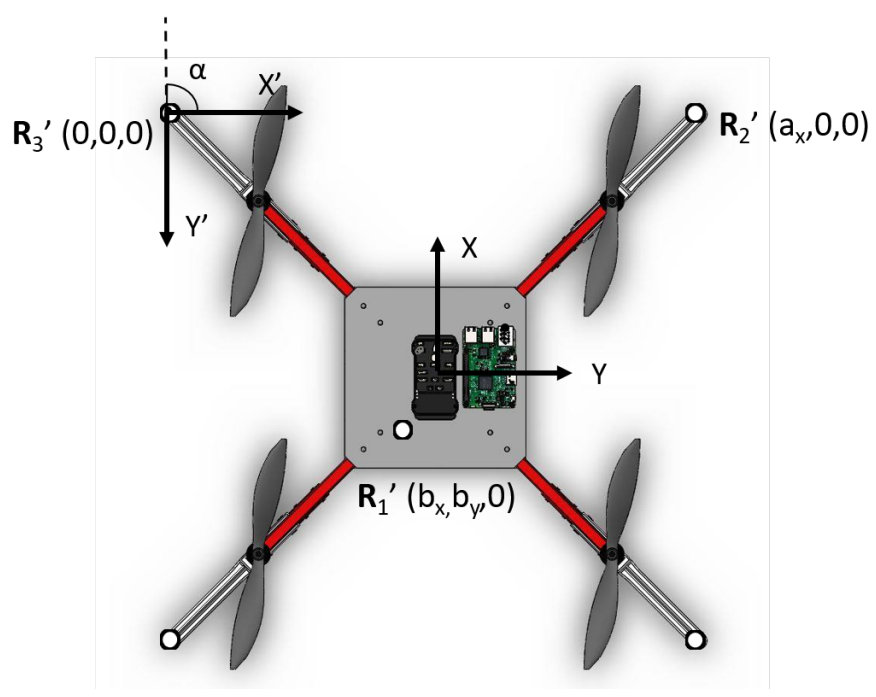

Figure 4.22: Analytical local coordinate frame top view

1, 2, and 3 will be used for the derivation but this can be any combination of three receivers. The position of the beacon in the analytical local frame is denoted as, $\boldsymbol{P}^{\prime}$. The positions of each receiver defined in the analytical local frame are $\boldsymbol{R}_{\mathbf{3}}{ }^{\prime}, \boldsymbol{R}_{\mathbf{2}}{ }^{\prime}$ and $\boldsymbol{R}_{\mathbf{1}}{ }^{\prime}$. The analytical local frame must be created such that a receiver $\left(\boldsymbol{R}_{\mathbf{3}}{ }^{\prime}\right)$ is located at the origin as shown in Fig. 4.22. Another receiver $\left(\boldsymbol{R}_{\mathbf{2}}{ }^{\prime}\right)$ must be oriented on the $\mathrm{X}^{\prime}$ axis at distance, $a_{x}$, away as shown in Fig. 4.22. The final receiver $\left(\boldsymbol{R}_{\mathbf{1}}{ }^{\prime}\right)$ must lie on the $\mathrm{X}^{\prime} \mathrm{Y}^{\prime}$ plane as previously shown in Fig. 4.22 and Fig. 4.23. The distances, $\mathrm{d}$, are measured through the ToF as shown in Eq. 4.2. The receiver order is selected such that a corner receiver is selected as the analytical frame origin and the axes are aligned such that the Z' axis points close to downward but slightly offset since $\boldsymbol{R}_{\mathbf{1}}$ is mounted beneath the other receivers.

The spherical surface equations can be written for receivers 3,2 , and 1 in the analytical local frame as follows:

$$
x_{p^{\prime}}^{2}+y_{p^{\prime}}^{2}+z_{p^{\prime}}^{2}=d_{3}^{2}
$$




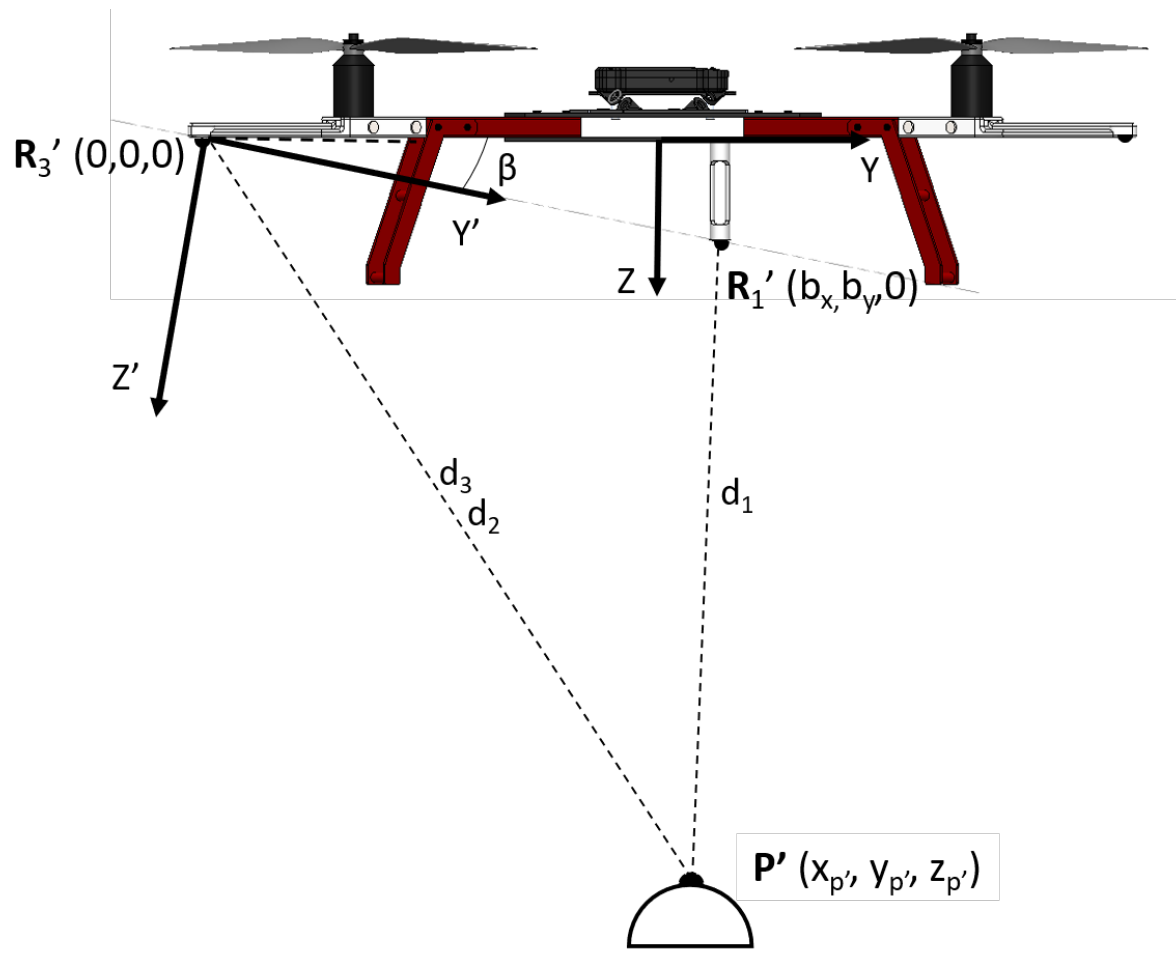

Figure 4.23: Analytical local coordinate frame side view

$$
\begin{gathered}
\left(x_{p^{\prime}}-a_{x}\right)^{2}+y_{p^{\prime}}^{2}+z_{p^{\prime}}^{2}=d_{2}^{2} \\
\left(x_{p^{\prime}}-b_{x}\right)^{2}+\left(y_{p^{\prime}}-b_{y}\right)^{2}+z_{p^{\prime}}^{2}=d_{1}^{2}
\end{gathered}
$$

The position of the beacon can be calculated through substitution and elimination. The $\mathrm{x}$ coordinate is solved by subtracting Eq. 4.9 from Eq. 4.10 and rearranging for $x_{p^{\prime}}$,

$$
x_{p^{\prime}}=\frac{d_{3}^{2}-d_{2}^{2}+a_{x}^{2}}{2 a_{x}}
$$

Subtracting Eq. 4.9 from Eq. 4.11 yields the position $y_{p^{\prime}}$ as,

$$
y_{p^{\prime}}=\frac{d_{3}^{2}-d_{1}^{2}+b_{x}^{2}+b_{y}^{2}-2 b_{x} x_{p^{\prime}}}{2 b_{y}}
$$


Now substituting the result for $x_{p^{\prime}}$ from Eq. 4.12 ,

$$
y_{p^{\prime}}=\frac{d_{3}^{2}-d_{1}^{2}+b_{x}^{2}+b_{y}^{2}}{2 b_{y}}-\frac{b_{x}\left(d_{3}^{2}-d_{2}^{2}+a_{x}^{2}\right)}{2 a_{x} b_{y}}
$$

The $z_{p^{\prime}}$ coordinate can then be solved by rearranging Eq. 4.9 for $z_{p^{\prime}}$ and using the previous results (Eq's 4.12 and 4.13) to solve,

$$
z_{p^{\prime}}=+\sqrt{d_{3}^{2}-x_{p^{\prime}}^{2}-y_{p^{\prime}}^{2}}
$$

The position coordinates of the beacon must be translated and rotated from the analytical local frame to the quadrotor local frame. The analytical frame must first rotate $\beta$ degrees around the $\mathrm{X}^{\prime}$ axis and then must rotate $\alpha$ degrees around the Z' axis. Next it must translate from $\boldsymbol{R}_{3}$ defined in the quadrotor local frame to the origin of the quadrotor local frame. These transformations are shown in Eq. (4.16).

$$
\boldsymbol{P}=\boldsymbol{W}_{z}(\alpha) \boldsymbol{W}_{x}(\beta) \boldsymbol{P}^{\prime}+\boldsymbol{R}_{3}
$$

The rotation matrices $W_{x}$ and $W_{z}$ can be written as:

$$
\begin{aligned}
& \boldsymbol{W}_{z}(\alpha)=\left[\begin{array}{ccc}
\cos (\alpha) & \sin (\alpha) & 0 \\
-\sin (\alpha) & \cos (\alpha) & 0 \\
0 & 0 & 1
\end{array}\right] \\
& \boldsymbol{W}_{x}(\beta)=\left[\begin{array}{ccc}
1 & 0 & 0 \\
0 & \cos (\beta) & \sin (\beta) \\
0 & -\sin (\beta) & \cos (\beta)
\end{array}\right]
\end{aligned}
$$

The angles $\alpha$ and $\beta$ can be calculated according to geometry as follows (based on the quadrotor local frame): 


$$
\begin{aligned}
& \alpha=\operatorname{atan}\left(\frac{x_{r 3}-x_{r 2}}{y_{r 3}-y_{r 2}}\right) \\
& \beta=\operatorname{atan}\left(\frac{y_{r 3}-y_{r 1}}{z_{r 3}-z_{r 1}}\right)
\end{aligned}
$$

A summary of the receiver combinations and the rotation angles is listed in Table 4.3. The first receiver listed is where the origin of the analytical local frame is placed, the second receiver is seperated by a distance, $a_{x}$, along the $\mathrm{X}^{\prime}$ axis, and the third receiver listed is located in the $\mathrm{X}^{\prime} \mathrm{Y}^{\prime}$ plane.

Table 4.3: Summary of the analytical method rotation angles

\begin{tabular}{|c|c|c|}
\hline Receivers & $\alpha$ & $\beta$ \\
\hline \hline $3,2,1$ & $10.1^{\circ}$ & $90^{\circ}$ \\
\hline $2,5,1$ & $13.6^{\circ}$ & $180^{\circ}$ \\
\hline $5,4,1$ & $14.8^{\circ}$ & $270^{\circ}$ \\
\hline $4,3,1$ & $10.7^{\circ}$ & $0^{\circ}$ \\
\hline $3,2,5$ & $0^{\circ}$ & $90^{\circ}$ \\
\hline $3,2,4$ & $0^{\circ}$ & $90^{\circ}$ \\
\hline $5,4,2$ & $0^{\circ}$ & $270^{\circ}$ \\
\hline $5,4,3$ & $0^{\circ}$ & $270^{\circ}$ \\
\hline
\end{tabular}

There are two combinations which are not used, $\boldsymbol{R}_{3}, \boldsymbol{R}_{5}, \boldsymbol{R}_{1}$ and $\boldsymbol{R}_{2}, \boldsymbol{R}_{4}, \boldsymbol{R}_{1}$. These two combinations nearly form a line in the analytical local frame due to the 
geometry (see Fig. 4.3). This leads to larger calculated position errors.

\subsubsection{Linear Least Squares Method}

The LLS method can be completed using the quadrotor local frame. To perform LLS the nonlinear equation of a sphere, given by Eq. 4.4, must be linearized. This can be simplified to a linear equation by taking $\boldsymbol{R}_{1}$ as a reference node and subtracting it from the other receiver equations as shown below,

$$
\begin{gathered}
\left(x_{p}-x_{r i}\right)^{2}+\left(y_{p}-y_{r i}\right)^{2}+\left(z_{p}-z_{r i}\right)^{2}+e_{i}^{2}-\left(\left(x_{p}-x_{r 1}\right)^{2}+\left(y_{p}-y_{r 1}\right)^{2}+\left(z_{p}-z_{r 1}\right)^{2}+e_{1}^{2}\right) \\
=d_{i}^{2}-d_{1}^{2}, \quad i \in[2, n]
\end{gathered}
$$

The only case where $\boldsymbol{R}_{1}$ can not be used as a reference node is in the case using the four corner receivers $\left(\boldsymbol{R}_{2}, \boldsymbol{R}_{3}, \boldsymbol{R}_{4}, \boldsymbol{R}_{5}\right)$ for LLS. This case is unique and is covered after introducing the general approach which is used in the other cases. This linearization increases the minimum number of required receivers to four to solve for the three unknowns $x_{p}, y_{p}$, and $z_{p}$. For four receiver LLS $\mathrm{n}=4$, and for five receiver LLS $\mathrm{n}$ $=5$. This equation simplifies as follows, where the unknowns $x_{p}, y_{p}$, and $z_{p}$ are all linear.

$$
\begin{aligned}
& x_{p} \cdot\left(x_{r 1}-x_{r i}\right)+y_{p} \cdot\left(y_{r 1}-y_{r i}\right)+z_{p} \cdot\left(z_{r 1}-z_{r i}\right)+e_{i}^{2}-e_{1}^{2} \\
= & 0.5 \cdot\left(x_{r 1}-x_{r i}+y_{r 1}-y_{r i}+z_{r 1}-z_{r i}+d_{i}^{2}-d_{1}^{2}\right), i \in[2, n]
\end{aligned}
$$

On the right hand side of the equal sign all of the values are known through geometry or the measured ToF. The resulting system of equations is shown in Eq. 4.23, where $\boldsymbol{P}$ is the desired position in Eq. 4.24, $\boldsymbol{A}$ is a matrix of coefficients for the position given in Eq. 4.25, $\boldsymbol{E}$ is a matrix of unknown measurement errors given in Eq. 4.26 and $\boldsymbol{b}$ is a matrix of known values given by Eq. 4.27 [43]. 


$$
\begin{aligned}
& \boldsymbol{A P}+\boldsymbol{E}=\boldsymbol{b} \\
& \boldsymbol{P}=\left[x_{p}, y_{p}, z_{p}\right]^{T} \\
& \boldsymbol{A}=\left[\begin{array}{ccc}
x_{r 1}-x_{r 2} & y_{r 1}-y_{r 2} & z_{r 1}-z_{r 2} \\
\vdots & \vdots & \vdots \\
x_{r 1}-x_{r n} & y_{r 1}-y_{r n} & z_{r 1}-z_{r n}
\end{array}\right] \\
& \boldsymbol{E}=\left[\begin{array}{c}
e_{2}^{2}-e_{1}^{2} \\
\vdots \\
e_{n}^{2}-e_{1}^{2}
\end{array}\right] \\
& \boldsymbol{b}=\left[\begin{array}{c}
0.5 \cdot\left(x_{r 1}-x_{r 2}+y_{r 1}-y_{r 2}+z_{r 1}-z_{r 2}+d_{2}^{2}-d_{1}^{2}\right) \\
\vdots \\
0.5 \cdot\left(x_{r 1}-x_{r n}+y_{r 1}-y_{r n}+z_{r 1}-z_{r n}+d_{n}^{2}-d_{1}^{2}\right)
\end{array}\right]
\end{aligned}
$$

The goal is to solve for the best position which minimizes the error. This will require matrix manipulation, the following properties are used during the derivation. Where $\boldsymbol{A}$ and $\boldsymbol{B}$ are matrices and $k$ is a scalar value.

$$
\boldsymbol{A}^{T^{T}}=\boldsymbol{A}
$$

$$
(\boldsymbol{A}+\boldsymbol{B})^{T}=\boldsymbol{A}^{T}+\boldsymbol{B}^{T}
$$


$(\boldsymbol{A} \boldsymbol{B})^{T}=\boldsymbol{B}^{T} \boldsymbol{A}^{T}$

$$
(k)^{T}=k
$$

First Eq. 4.23 is rearranged for the error shown as,

$$
E=b-A P
$$

The squared error is represented by $J$, this is the cost function which is minimized. $\mathrm{J}$ is a scalar value, $\boldsymbol{E}$ is a $\mathrm{nx} 1$ vector, $\boldsymbol{b}$ has dimensions $\mathrm{nx} 1, \boldsymbol{A}$ has dimensions $\mathrm{nx} 3$ and $\boldsymbol{P}$ has dimensions $3 \times 1$.

$$
J=\boldsymbol{E}^{T} \boldsymbol{E}=\left(\boldsymbol{b}^{T}-\boldsymbol{P}^{T} \boldsymbol{A}^{T}\right)(\boldsymbol{b}-\boldsymbol{A P})
$$

This equation is expanded to,

$$
J=\underbrace{\boldsymbol{b}^{T} \boldsymbol{b}}_{J_{1}}-\underbrace{2 \boldsymbol{P}^{T} \boldsymbol{A}^{T} \boldsymbol{b}}_{J_{2}}+\underbrace{\boldsymbol{P}^{T} \boldsymbol{A}^{T} \boldsymbol{A} \boldsymbol{P}}_{J_{3}}
$$

where,

$$
\boldsymbol{b}^{T} \boldsymbol{A} \boldsymbol{P}=\boldsymbol{b} \boldsymbol{P}^{T} \boldsymbol{A}^{T}
$$

by virtue of Eq's 4.30 and 4.31. The error is a minimum if the derivative of $J$ with respect to the position is zero (and the second derivative is positive),

$$
\frac{\partial J}{\partial \boldsymbol{P}}=\frac{\partial J_{1}}{\partial \boldsymbol{P}}+\frac{\partial J_{2}}{\partial \boldsymbol{P}}+\frac{\partial J_{3}}{\partial \boldsymbol{P}}=0
$$

The first term, $\partial J_{1} / \partial \boldsymbol{P}$, is a constant with respect to the position, since the position of the beacon, $\boldsymbol{P}=\left(x_{p}, y_{p}, z_{p}\right)$ does not appear in Eq. 4.27 thus, 


$$
\frac{\partial J_{1}}{\partial \boldsymbol{P}}=\left[\begin{array}{l}
\frac{\partial J_{1}}{\partial x_{p}} \\
\frac{\partial J_{1}}{\partial y_{p}} \\
\frac{\partial J_{1}}{\partial z_{p}}
\end{array}\right]=0
$$

For the second term, if we let $\boldsymbol{A}^{T} \boldsymbol{b}=\left(c_{1}, c_{2}, c_{3}\right)^{T}$ then we can write,

$$
J_{2}=-2\left(x_{p} c_{1}+y_{p} c_{2}+z_{p} c_{3}\right)
$$

The terms $c_{1}, c_{2}$, and $c_{3}$ are constant with respect to $\boldsymbol{P}$ and thus can write,

$$
\frac{\partial J_{2}}{\partial \boldsymbol{P}}=\left[\begin{array}{l}
\frac{\partial J_{2}}{\partial x_{p}} \\
\frac{\partial J_{2}}{\partial y_{p}} \\
\frac{\partial J_{2}}{\partial z_{p}}
\end{array}\right]=-2\left[\begin{array}{l}
c_{1} \\
c_{2} \\
c_{3}
\end{array}\right]=-2 \boldsymbol{A}^{T} \boldsymbol{b}
$$

For the remaining term, $J_{3}$, noting that the product $\boldsymbol{A}^{T} \boldsymbol{A}$ can be expressed as (using Eqs 4.28 and 4.30),

$$
\begin{gathered}
\left(\boldsymbol{A}^{T} \boldsymbol{A}\right)^{T}=\boldsymbol{A}^{T} \boldsymbol{A} \\
\boldsymbol{A}^{T} \boldsymbol{A}=\left[\begin{array}{lll}
a_{11} & a_{12} & a_{13} \\
a_{12} & a_{22} & a_{23} \\
a_{13} & a_{23} & a_{33}
\end{array}\right]
\end{gathered}
$$

Where $\mathrm{a}$ is a constant which depends on the receiver positions on the quadrotor. Multiplying by the position yields, 


$$
\boldsymbol{A}^{T} \boldsymbol{A P}=\left[\begin{array}{c}
a_{11} x_{p}+a_{12} y_{p}+a_{13} z_{p} \\
a_{12} x_{p}+a_{22} y_{p}+a_{23} z_{p} \\
a_{13} x_{p}+a_{23} y_{p}+a_{33} z_{p}
\end{array}\right]
$$

Using this result, the entire term $J_{3}$ can be wrriten as,

$$
\begin{gathered}
J_{3}=\boldsymbol{P}^{T} \boldsymbol{A}^{T} \boldsymbol{A} \boldsymbol{P} \\
J_{3}=a_{11} x_{p}^{2}+a_{22} y_{p}^{2}+a_{33} z_{p}^{2}+2 a_{12} x_{p} y_{p}+2 a_{13} x_{p} z_{p}+2 a_{23} y_{p} z_{p}
\end{gathered}
$$

thus the derivative becomes,

$$
\frac{\partial J_{3}}{\partial \boldsymbol{P}}=\left[\begin{array}{l}
\frac{\partial J_{3}}{\partial x_{p}} \\
\frac{\partial J_{3}}{\partial y_{p}} \\
\frac{\partial J_{3}}{\partial z_{p}}
\end{array}\right]=\left[\begin{array}{l}
2 a_{11} x_{p}+2 a_{12} y_{p}+2 a_{13} z_{p} \\
2 a_{12} x_{p}+2 a_{22} y_{p}+2 a_{23} z_{p} \\
2 a_{13} x_{p}+2 a_{23} y_{p}+2 a_{33} z_{p}
\end{array}\right]=2 \boldsymbol{A}^{T} \boldsymbol{A P}
$$

Combining Eq's 4.37, 4.39, and 4.45 and setting to zero,

$$
\frac{\partial J}{\partial \boldsymbol{P}}=0=-2 \boldsymbol{A}^{T} \boldsymbol{b}+2 \boldsymbol{A}^{T} \boldsymbol{A P}
$$

which can be rearranged to solve for $\boldsymbol{P}$,

$$
\boldsymbol{P}=\left(\boldsymbol{A}^{T} \boldsymbol{A}\right)^{-1}\left(\boldsymbol{A}^{T} \boldsymbol{b}\right)
$$

This expression allows the position of the beacon, $\boldsymbol{P}$, to be solved with any number of receivers greater than or equal to four. For this to be a minimum the second derivative can be found from Eq. 4.47 as, 


$$
\frac{\partial J^{2}}{\partial P^{2}}=2 \boldsymbol{A}^{T} \boldsymbol{A}
$$

If $\boldsymbol{A}$ is positive definite (which is ensured if $\boldsymbol{A}$ has full rank) then the second derivative will be greater than zero and thus $\boldsymbol{P}$ can be solved. $\boldsymbol{A}$ is ensured full rank since all the receivers are not located on a single plane.

To estimate the standard error of the least squares regression the Student's t-test statistical hypothesis is selected. This hypothesis is applicable because the error is assumed to be white gaussian, equal across all receivers and the number of receivers represent a sample. The standard error of the slope of a regression line is proportional to,

$$
S E(\boldsymbol{P}) \propto \sqrt{\frac{1}{n-4}}
$$

where n-4 represents the degrees of freedom (three unknowns and the first receiver required to linearize). Therefore increasing the number of receivers from 4 to 5 should decrease the standard error of the calculated position by a factor of $\sqrt{2}$. If TDoA is used the number of required measurements is 5 , thus the degree of freedom using five receivers is 1 and a less accurate measurement is obtained.

In the case where the four corner receivers are used for LLS, these are all mounted on the same plane (see Fig. 4.3). The simplified linearized spherical equation from Eq.4.22 can be rewritten as,

$$
\begin{gathered}
x_{p} \cdot\left(x_{r 2}-x_{r i}\right)+y_{p} \cdot\left(y_{r 2}-y_{r i}\right)+e_{i}^{2}-e_{2}^{2} \\
=0.5 \cdot\left(x_{r 2}-x_{r i}+y_{r 2}-y_{r i}+d_{i}^{2}-d_{2}^{2}\right), \quad i \in[3,5]
\end{gathered}
$$

where $z_{r i}=z_{r 1}=0$ for all receivers, thus $z_{p}$ cannot be solved for. The lateral position can still be calculated because the position that yields the minimum error squared will be the lateral position $\left(x_{p}, y_{p}\right)$. Since $\boldsymbol{R}_{1}$ is not used in this calculation, $\boldsymbol{R}_{2}$ is 
used for linearization instead. Since $z_{p}$ is no longer a variable the matrices decrease in size. The least squares problem can be rewritten as follows,

$$
\begin{gathered}
\boldsymbol{P}_{\text {plane }}=\left[x_{p}, y_{p}\right]^{T} \\
\boldsymbol{A}_{\text {plane }}=\left[\begin{array}{ll}
x_{r 2}-x_{r 3} & y_{r 2}-y_{r 3} \\
x_{r 2}-x_{r 4} & y_{r 2}-y_{r 4} \\
x_{r 2}-x_{r 5} & y_{r 2}-y_{r 5}
\end{array}\right] \\
\boldsymbol{E}_{p l a n e}=\left[\begin{array}{l}
e_{3}^{2}-e_{2}^{2} \\
e_{4}^{2}-e_{2}^{2} \\
e_{5}^{2}-e_{2}^{2}
\end{array}\right] \\
\boldsymbol{b}_{\text {plane }}=\left[\begin{array}{c}
0.5 \cdot\left(x_{r 2}-x_{r 3}+y_{r 2}-y_{r 4}+d_{4}^{2}-d_{2}^{2}\right) \\
0.5 \cdot\left(x_{r 2}-x_{r 2}+y_{r 2}-y_{r 3}+d_{3}^{2}-d_{2}^{2}\right) \\
\left.0.5 \cdot x_{r 2}-x_{r 4}+y_{r 2}-y_{r 5}+d_{5}^{2}-d_{2}^{2}\right)
\end{array}\right]
\end{gathered}
$$

where plane denotes the four receivers form a plane. This system of equations can be solved using only three receivers since there are two unknowns with an additional receiever required for linearization.

\subsubsection{Dilution of Precision}

Dilution of precision (DoP) is a method to categorize how accurate a range measurement is, mostly used for GPS measurements. The geometry of the receivers can 


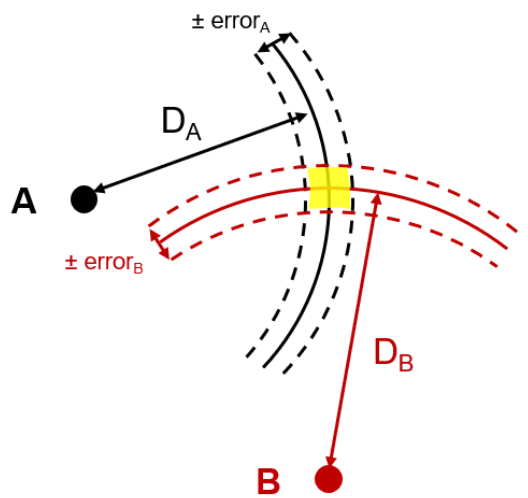

Figure 4.24: Low GDoP

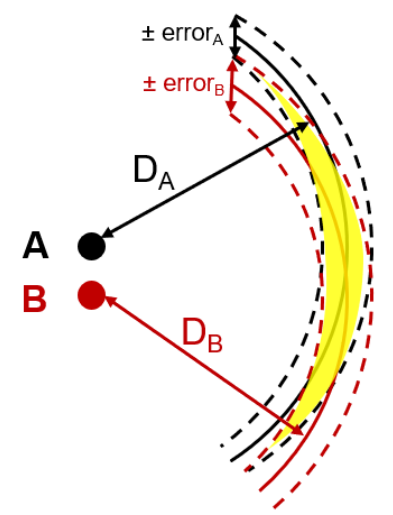

Figure 4.25: High GDoP

impact the accuracy of the receivers through the DOP. A 2D example of a low DoP is shown in Fig. 4.24, the solid lines show the distance measurement from each receiver and the dashed lines show the error in each measurement. The intersection of the two solid lines shows the position of the beacon. The shaded area is the position uncertainty defined by the measurement uncertainty from receiver A and B. By having the two measurements intersect perpendicularly a low DOP is achieved. A high DOP is shown in Fig. 4.25. The two receivers are located next to each other and the position uncertainty has significantly increased while maintaining the same measurement uncertainty.

There are two types of errors which can lead to inaccuracies, geometry related or distance related. The geometry error is based on inaccuracies measuring the receiver exact position and possible wing flex during flight. This will effect the calculated beacon position as it relies on accurate receiver positions. The true beacon position, $\hat{\boldsymbol{P}}$, can be represented by the calculated position, $\boldsymbol{P}$, and the error, $\Delta \boldsymbol{P}$.

$$
\hat{\boldsymbol{P}}=\boldsymbol{P}+\Delta \boldsymbol{P}
$$


Similarly, distance related errors consist of turbulence, air temperature, and vibrations. The true distance measurement, $\hat{d}$, can be represented by the calculated distance, $d$, and the error, $\Delta d$.

$$
\hat{d}_{i}=d_{i}+\Delta d_{i}, i \in[1,5]
$$

The DoP calculation assumes that the calculated position of the beacon, $\boldsymbol{P}$, the position of the receivers, $\boldsymbol{R}$, and the calculated distances from Eq. 4.2 are known. Let $\mu_{r i}$ denote the unit vector between the beacon and a receiver where all values are known,

$$
\mu_{r i}=\left(\frac{\left(x_{r i}-x_{p}\right)}{d_{i}}, \frac{\left(y_{r i}-y_{p}\right)}{d_{i}}, \frac{\left(z_{r i}-z_{p}\right)}{d_{i}}\right), i \in[1,5]
$$

Typically for GPS calculations of DoP a time offset is included as a fourth variable, but this is not required because the clocks are synchronized. Let the true distance measurement from the beacon to each receiver be expressed in terms of the true position, $\hat{\boldsymbol{P}}$, and the unit vectors.

$$
\hat{d}_{i}=\hat{\mu}_{r i} \cdot \hat{\boldsymbol{P}}, i \in[1,5]
$$

Substituting in Eqs. 4.55 and 4.56,

$$
d_{i}+\Delta d_{i}=\hat{\mu}_{r i} \cdot \boldsymbol{P}+\hat{\mu}_{r i} \cdot \Delta \boldsymbol{P}, i \in[1,5]
$$

This expands to,

$$
\begin{gathered}
d_{i}+\Delta d_{i}=x_{p} \frac{\left(x_{r i}-x_{p}\right)}{d_{i}}+y_{p} \frac{\left(y_{r i}-y_{p}\right)}{d_{i}}+z_{p} \frac{\left(z_{r i}-z_{p}\right)}{d_{i}}+ \\
\Delta x_{p} \frac{\left(x_{r i}-x_{p}\right)}{d_{i}}+\Delta y_{p} \frac{\left(y_{r i}-y_{p}\right)}{d_{i}}+\Delta z_{p} \frac{\left(z_{r i}-z_{p}\right)}{d_{i}}+, i \in[1,5]
\end{gathered}
$$

where, 


$$
\boldsymbol{\Delta} \boldsymbol{P}=\left[\Delta x_{p}, \Delta y_{p}, \Delta z_{p}\right]^{T}
$$

Putting this into matrix form,

$$
D+\Delta D=C P+C \Delta P
$$

where $\boldsymbol{D}$ represents a vector of the estimated distance measurements,

$$
\boldsymbol{D}=\left[d_{1}, d_{2}, d_{3}, d_{4}, d_{5}\right]^{T}
$$

and $\boldsymbol{\Delta} \boldsymbol{D}$ represents a vector of the distance measurement errors,

$$
\Delta \boldsymbol{D}=\left[\Delta d_{1}, \Delta d_{2}, \Delta d_{3}, \Delta d_{4}, \Delta d_{5}\right]^{T}
$$

The matrix $\boldsymbol{C}$ represents the unit vectors from the beacon to each receiver,

$$
\boldsymbol{C}=\left[\begin{array}{lll}
\frac{\left(x_{r 1}-x_{p}\right)}{d_{1}} & \frac{\left(y_{r 1}-y_{p}\right)}{d_{1}} & \frac{\left(z_{r 1}-z_{p}\right)}{d_{1}} \\
\frac{\left(x_{r 2}-x_{p}\right)}{d_{2}} & \frac{\left(y_{r 2}-y_{p}\right)}{d_{2}} & \frac{\left(z_{r 2}-z_{p}\right)}{d_{2}} \\
\frac{\left(x_{r 3}-x_{p}\right)}{d_{3}} & \frac{\left(y_{r 3}-y_{p}\right)}{d_{3}} & \frac{\left(z_{r 3}-z_{p}\right)}{d_{3}} \\
\frac{\left(x_{r 4}-x_{p}\right)}{d_{4}} & \frac{\left(y_{r 4}-y_{p}\right)}{d_{4}} & \frac{\left(z_{r 4}-z_{p}\right)}{d_{4}} \\
\frac{\left(x_{r 5}-x_{p}\right)}{d_{5}} & \frac{\left(y_{r 5}-y_{p}\right)}{d_{5}} & \frac{\left(z_{r 5}-z_{p}\right)}{d_{5}}
\end{array}\right]
$$

Since the errors are unknown let us approximate the solution using only the calculated values,

$$
D=C P
$$

Where relating this to the previous least squares problem (Eq. 4.23), $\boldsymbol{D}=\boldsymbol{b}$ and $\boldsymbol{C}=\boldsymbol{A}$ and neglecting the error term. Therefore the least squares solution to this 
equation will be,

$$
\boldsymbol{P}=\left(\boldsymbol{C}^{T} \boldsymbol{C}\right)^{-1}\left(\boldsymbol{C}^{T} \boldsymbol{D}\right)
$$

Substituting this expression for $\boldsymbol{P}$ back into the Eq. 4.62 yields,

$$
\boldsymbol{D}+\boldsymbol{\Delta} \boldsymbol{D}=\boldsymbol{C}\left(\boldsymbol{C}^{T} \boldsymbol{C}\right)^{-1}\left(\boldsymbol{C}^{T} \boldsymbol{D}\right)+\boldsymbol{C} \boldsymbol{\Delta P}
$$

which simplifies to,

$$
\Delta D=C \Delta P
$$

based on the following properties of inverses where A and B are two arbitrary matrices and $\mathrm{I}$ is the identity matrix,

$$
\begin{gathered}
(\boldsymbol{A} \boldsymbol{B})^{-1}=\boldsymbol{B}^{-1} \boldsymbol{A}^{-1} \\
\boldsymbol{A} \boldsymbol{A}^{-1}=\boldsymbol{I} \\
\boldsymbol{A}^{T} \boldsymbol{A}^{T^{-1}}=\boldsymbol{I}
\end{gathered}
$$

Eq. 4.69 is in the same least squares form as Eq. 4.23 where $\boldsymbol{\Delta} \boldsymbol{D}=\boldsymbol{b}$ and $\boldsymbol{\Delta} \boldsymbol{C}=\boldsymbol{A}$ and neglecting the error term. Solving this equation for $\boldsymbol{\Delta} \boldsymbol{P}$ using the least squares solution (Eq. 4.47) yields,

$$
\boldsymbol{\Delta} \boldsymbol{P}=\left(\boldsymbol{C}^{T} \boldsymbol{C}\right)^{-1}\left(\boldsymbol{C}^{T} \boldsymbol{\Delta} \boldsymbol{D}\right)
$$

This solution will be used to find the DoP values but first variance $\left(\sigma^{2}\right)$ is defined. Variance is the square of the standard deviation $(\sigma)$. The dilutions of precision depend on determining the variance of the position $\left(\sigma_{x}^{2}, \sigma_{y}^{2}, \sigma_{z}^{2}\right)$. Co-variance is how 
the variables vary with each other such as how the $\mathrm{x}$ position increases when the $\mathrm{y}$ position increases. For this application the co-variance is nearly zero as each axis is independent. The co-variance matrix will be used to estimate the position variance. First the co-variance of the position is defined as,

$$
\operatorname{cov}(\boldsymbol{P})=\left\langle\boldsymbol{\Delta} \boldsymbol{P} \boldsymbol{\Delta} \boldsymbol{P}^{T}\right\rangle
$$

Where \langle\rangle denote the expectation operator. Substituting in Eq. 4.73 yields,

$$
\operatorname{cov}(\boldsymbol{P})=\left(\boldsymbol{C}^{T} \boldsymbol{C}\right)^{-1} \boldsymbol{C}^{T}\left\langle\boldsymbol{\Delta} \boldsymbol{D} \boldsymbol{\Delta} \boldsymbol{D}^{T}\right\rangle \boldsymbol{C}\left(\boldsymbol{C}^{T} \boldsymbol{C}\right)^{-1}
$$

If all the range measurement errors are assumed to have the same variance, $\sigma_{d}^{2}$, are uncorrelated, and are zero mean, then the expectation simplifies to,

$$
\left\langle\Delta \boldsymbol{D} \Delta \boldsymbol{D}^{T}\right\rangle=\sigma_{d}^{2}
$$

Substituting this results into Eq. 4.75 and simplifying,

$$
\operatorname{cov}(\boldsymbol{P})=\sigma_{d}^{2}\left(\boldsymbol{C}^{T} \boldsymbol{C}\right)^{-1}
$$

This expression can be solved for since the geometry of the receivers, the calculated position of the beacon and the distance measurements to each receiver are known. Solving this equation produces a $3 \times 3$ matrix which is equivalent to the covariance matrix. By definition the covariance matrix is,

$$
\operatorname{cov}(\boldsymbol{P})=\left[\begin{array}{ccc}
\sigma_{x}^{2} & \sigma_{x y} & \sigma_{x z} \\
\sigma_{x y} & \sigma_{y}^{2} & \sigma_{y z} \\
\sigma_{x z} & \sigma_{y z} & \sigma_{z}^{2}
\end{array}\right]
$$


Using this result the variances for the position can be identified along the diagonal. As previously mentioned the co-variances are nearly zero since each measurement should be independent. They are not exactly zero due to measurement errors. From this matrix the values for multiple measures of precision dilution can be obtained. These DoP calculations are geometry dependent and represent scaling factors for the range measurement variance, $\sigma_{d}^{2}$. Using four receivers, the ideal orientation which results in a PDoP of unity would be a tetrahedron surrounding the transmitter.

$$
\begin{aligned}
& \text { Position Dilution of Precision }=\sqrt{\sigma_{x}^{2}+\sigma_{y}^{2}+\sigma_{z}^{2}} \\
& \text { Horizontal Dilution of Precision }=\sqrt{\sigma_{x}^{2}+\sigma_{y}^{2}} \\
& \text { Vertical Dilution of Precision }=\sqrt{\sigma_{z}^{2}}
\end{aligned}
$$

The measured receiver distances have a standard deviation of $3.46 \mathrm{~cm}\left(\sigma_{d}^{2}\right)$, with the beacon stationary at $(0,0,177)$. The HDoP is calculated at different locations and the estimated horizontal standard deviation is shown in Fig. 4.26. The figure shows the beacon location at different $(\mathrm{X}, \mathrm{Y})$ positions relative to the quadrotor centre. For all of these locations the height is held constant at $177 \mathrm{~cm}$. There are nine nominal positions tested shown with letters A-I tested at three different radii $(0 \mathrm{~cm}, 50 \mathrm{~cm}$, and $100 \mathrm{~cm}$ ) shown by the dashed lines. A schematic of the quadrotor is shown in the top left for scale purposes and to provide the orientation. The arrow indicates the front of the quadrotor and each receiver is labelled, this is a bottom view since the $\mathrm{Z}$ axis is out of the page (whereas Fig. 4.4 is the top view).

The calculated horizontal (XY plane) standard deviation for each position based on the HDoP is shown using the dotted circles, the values are displayed to the top right above each position. As the beacon moves further away the HDoP increases because 
the direction vectors become closer to coincident. Underneath the HDoP values are the VDoP values which are lower than the HDoP values due to the geometry receivers. The average horizontal standard deviation of $31.6 \mathrm{~cm}$ is provided in the top right by taking the root mean square of the horizontal standard deviation values. The HDoP values are significantly higher than the $\mathrm{VDoP}$ values due to the geoemetry of the receivers as represented in Figure 4.25. If the height is increased the values for the HDoP increase and the values for VDoP remain similar. By mounting the corner receivers as far away as possible, this gives the lowest possible DoP. If the corner receivers are mounted half way along each arm the DoP values increase.

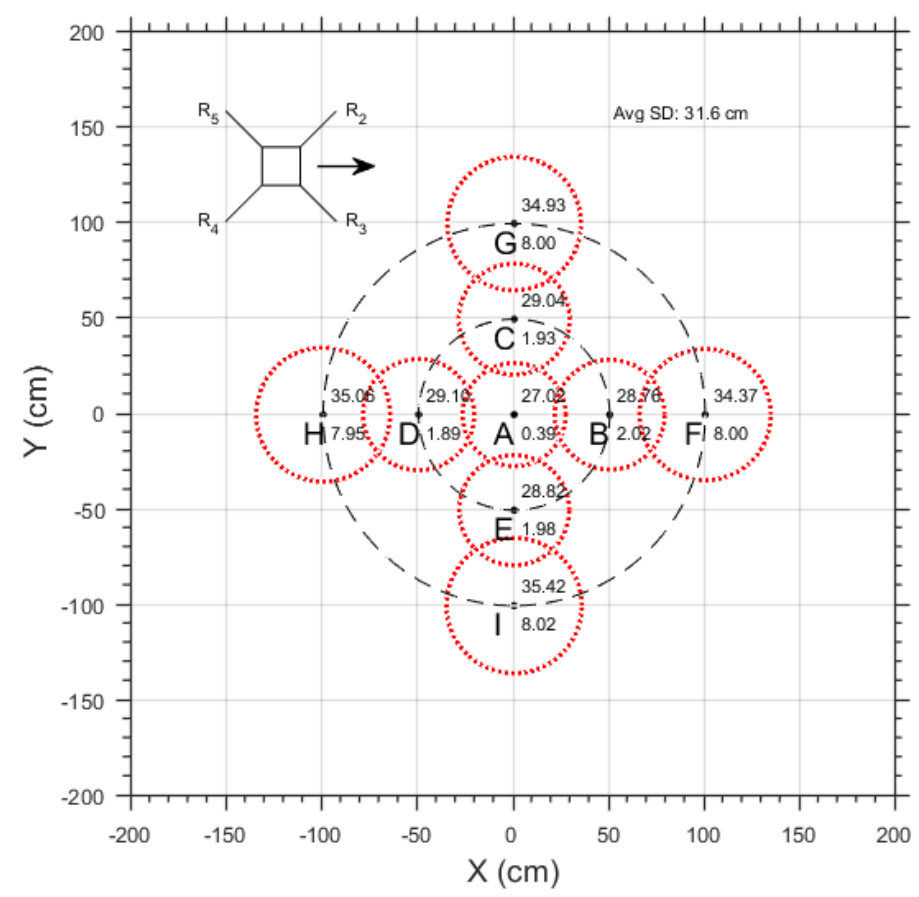

Figure 4.26: Dilution of precision at different locations

The system has a potential for high vertical accuracy directly over the beacon $(0.39 \mathrm{~cm})$ but this increases as the radius increases. To improve the vertical accuracy a downward facing sonar is used to obtain the height. Interference with the lateration 
system is avoided through time division multiple access (TDMA). The sonar measures the return time of flight of the signal. The MaxBotix MB1240 has the narrowest beam pattern which decreases scatter caused by the turbulence of the rotors. It has a maximum detection range of $7.65 \mathrm{~m}$ due to the attenuation of the ultrasonic wave. The sensor operates at $42 \mathrm{kHz}$ with a $1 \mathrm{~cm}$ resolution. The maximum update rate is $10 \mathrm{~Hz}$, limited by the decay time of the ultrasonic wave [69]. The measurement from the sonar is denoted as, $z_{s}$. When referring to specifically the vertical sonar it will be called the sonar. The ultrasonic beacon and receiver system is referred to as the lateration system and both of these combined make up the ULS.

In the case where the four receivers are mounted on the plane the vertical height can not be calculated through LLS. The vertical sonar allows the height to be calculated in this case.

\subsubsection{Kalman Filter}

A Kalman filter is applied to decrease real time noise in the position data. The Kalman filter is a set of recursive mathematical equations used to estimate a state vector while minimizing the mean error squared. The state vector is represented by $\boldsymbol{X}_{k}$, which in this application are the Kalman filtered positions $\left(x_{k}, y_{k}, z_{k}\right)$, and the Kalman filtered velocities $\left(\dot{x_{k}}, \dot{y_{k}}, \dot{z_{k}}\right)$.

$$
\boldsymbol{X}_{k}=\left[x_{k}, \dot{x}_{k}, y_{k}, \dot{y}_{k}, z_{k}, \dot{z}_{k}\right]^{T}
$$

The first step for a Kalman filter problem is to predict the future states based on the current states, these states are represented by,

$$
\boldsymbol{X}_{k+1}=\left[x_{k+1}, \dot{x}_{k+1}, y_{k+1}, \dot{y}_{k+1}, z_{k+1}, \dot{z}_{k+1}\right]^{T}
$$

To predict the future state, the process gain model is chosen as the random force 
model [70]. The equations are provided for the $\mathrm{X}$ axis, similarly these can be written for the two other axes,

$$
\begin{gathered}
x_{k+1}=x_{k}+\dot{x}_{k} d t+\frac{1}{2} w_{k} d t^{2} \\
\dot{x}_{k+1}=\dot{x}_{k}+w_{k} d t
\end{gathered}
$$

Where dt represents the change in time between steps. The acceleration is represented by $w_{k}$. It is assumed that the quadrotor is moving slow during hover and landing and thus is not accelerating over the time-step and any acceleration is assumed to be noise. At high accelerations this model is not applicable. The process noise is assumed to be zero-mean, independent and white Gaussian.

This can be written in matrix form to provide the general Kalman filter process model,

$$
\boldsymbol{X}_{k+1}=\boldsymbol{B}_{k} \boldsymbol{x}_{k}+\boldsymbol{G}_{k}
$$

Where the state transition matrix is described by $\boldsymbol{B}_{k}$, and the vector $\boldsymbol{G}_{k}$ represents the process noise matrix.

$$
\boldsymbol{B}_{k}=\left[\begin{array}{llllll}
1 & d t & 0 & 0 & 0 & 0 \\
0 & 1 & 0 & 0 & 0 & 0 \\
0 & 0 & 1 & d t & 0 & 0 \\
0 & 0 & 0 & 1 & 0 & 0 \\
0 & 0 & 0 & 0 & 1 & d t \\
0 & 0 & 0 & 0 & 0 & 1
\end{array}\right]
$$




$$
\boldsymbol{G}_{k}=\left[\begin{array}{c}
w_{k} \cdot d t^{2} / 2 \\
w_{k} \cdot d t \\
w_{k} \cdot d t^{2} / 2 \\
w_{k} \cdot d t \\
w_{k} \cdot d t^{2} / 2 \\
w_{k} \cdot d t
\end{array}\right]
$$

The next step is to update the prediction using measurements. The measurements consists of the lateral position from lateration, $\left(x_{p}, y_{p}\right)$ and the vertical height from the sonar, $z_{s}$. The velocities are not measured but are observed through the position states. The measurement vector is represented by $\boldsymbol{\zeta}_{k}$,

$$
\boldsymbol{\zeta}_{k}=\left[x_{p}, y_{p}, z_{s}\right]^{T}
$$

The measurements connect to the states through the Kalman filter measurement model,

$$
\boldsymbol{\zeta}_{k}=\boldsymbol{H}_{k} \boldsymbol{x}_{k+1}+\boldsymbol{V}_{k}
$$

Where the observation matrix is described by $\boldsymbol{H}_{k}$ and the measurement noise matrix is expressed by $\boldsymbol{V}_{k}$. The observation matrix to convert the states into the measurements is given by, 


$$
\boldsymbol{H}_{k}=\left[\begin{array}{llllll}
1 & 0 & 0 & 0 & 0 & 0 \\
0 & 0 & 1 & 0 & 0 & 0 \\
0 & 0 & 0 & 0 & 1 & 0
\end{array}\right]
$$

The measurement noise matrix is provided by,

$$
\boldsymbol{V}_{k}=\left[u_{k}, u_{k}, \epsilon_{k}\right]^{T}
$$

Where $u_{k}$ is the measurement noise for the multilateration equations and is initially set as $31.6 \mathrm{~cm}$ based on the average standard deviation from the DoP calculation (Fig.4.26) but changes based on the lateration algorithm. The measurement noise for the multilateration positions $\left(x_{p}, y_{p}\right)$ are assumed to be equivalent. The sonar measurement noise is represented by, $\epsilon_{k}$, and is experimentally measured to be $0.28 \mathrm{~cm}$ at a height of $177 \mathrm{~cm}$. The noise is assumed to be zero-mean, independent and white Gaussian.

\subsubsection{Algorithms}

A block diagram for the analytical method is shown in Fig. 4.27. It considers that only three receivers are connected even though there is a potential of five receivers. When this algorithm is implemented these are the only three receivers mounted on the quadrotor and the other receivers are mounted afterwards, these three receivers are $\boldsymbol{R}_{2}, \boldsymbol{R}_{3}$, and $\boldsymbol{R}_{4}$ (shown in Fig. 4.3). The PSoC starts the cycle by requesting an ultrasonic signal and waits until all three measurements are received. Once three measurements are received the distance is calculated by Eq. 4.2. The first filter ensures the measurement is positive and less than the maximum detection distance of $5 \mathrm{~m}$. This distance is selected as less than the maximum theoretical distance as 
external influences are not considered in the calculation. The second filter ensures the distance measurements are possible. The maximum relative measurement difference is the distance between the two receivers. This occurs when the signal propagates on the path connecting the two receivers. In this system it is not possible because the signal is limited to a beam angle of $45^{\circ}$, but this constraint is left due to measurement errors. This filter is applied to every distance measurement, $d_{i}$, against every other measurement, $d_{j}$. If the measurements pass the filters, the position is calculated using the analytical method described in Eq's 4.9 - 4.20. The height is measured using the sonar by triggering a pulse at a delayed time to avoid interference. The final measurement filter checks the calculated position is within a $45^{\circ}$ cone of the beacon. If the measurements fail any filter or the timer expires, the measurements are unsuccessful. The sonar height is always measured as it is independent of the distance measurement filters. A Kalman filter is used to reduce the noise on the calculated position and height before it is sent to the RPi. If the measurement passes all the filters it is considered successful as shown by the shaded boxes.

The next configuration adds another receiver on the remaining arm, the four receivers mounted on the quadrotor in this configuration are the four corner receivers $\left(\boldsymbol{R}_{2}, \boldsymbol{R}_{3}, \boldsymbol{R}_{4}\right.$, and $\left.\boldsymbol{R}_{5}\right)$. A similar block diagram is shown in Fig. 4.28 where the position is calculated using the LLS method with two significant differences. The first difference is that four measurements are required instead of three. The second is that LLS is used for localization instead of trilateration.

The final configuration adds the final receiver in the middle, $\boldsymbol{R}_{1}$, for five receiver LLS to increase accuracy. The same block diagram applies when using four or five receivers except the five receivers requires five successful distance measurements. 


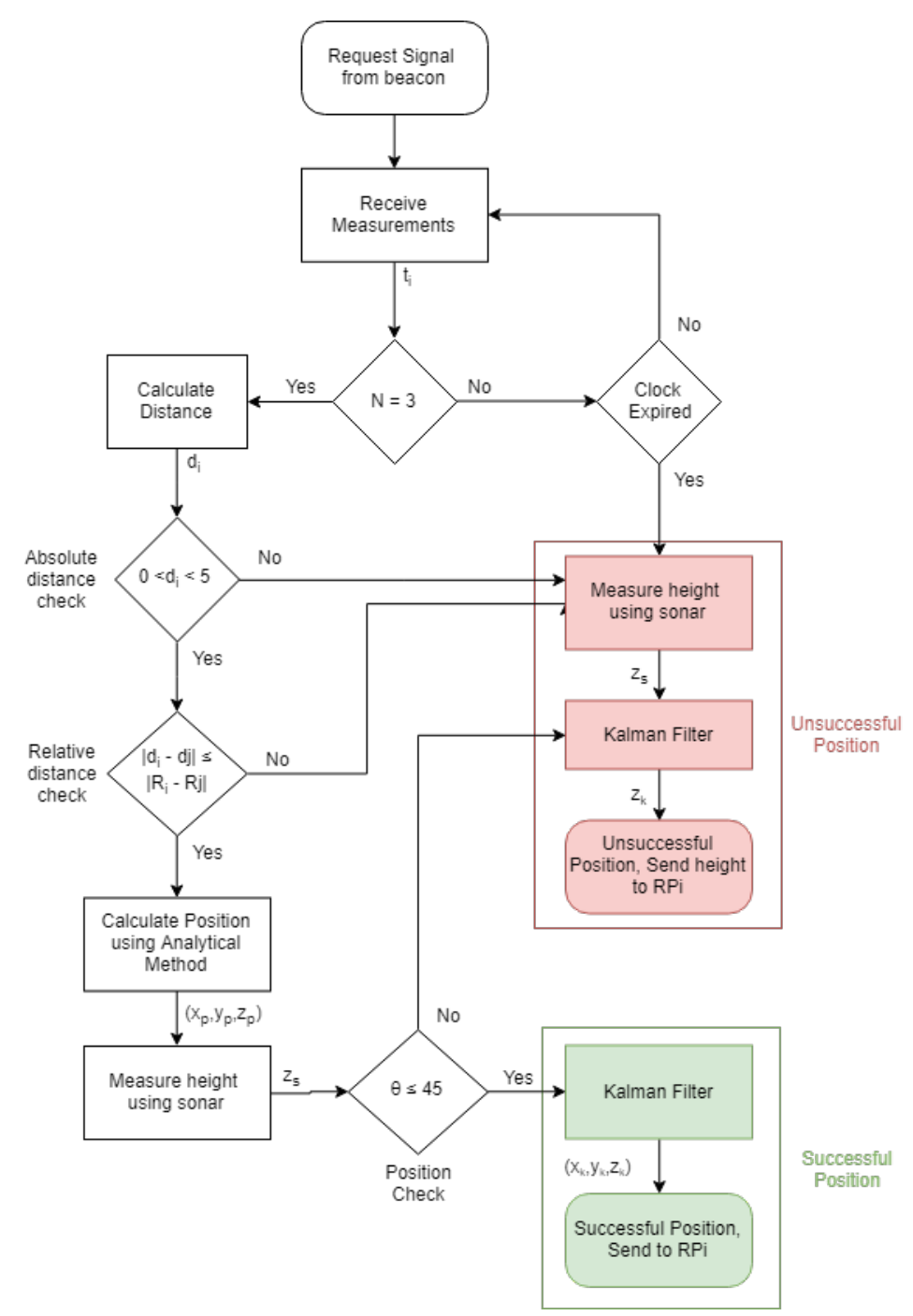

Figure 4.27: PSoC analytical method block diagram

\section{Loop Rate}

The ultrasonic transmission rate is tested to determine if multipath propagation between the chosen 10 period chirps influences the position accuracy. Figure 4.29 shows the effect of the update rate on both the standard deviation in the position estimate over $30 \mathrm{~s}$ and the number of successful measurements obtained. The measurements are tested with the beacon and quadrotor fixed at a position of $(100,0,177)$. For 


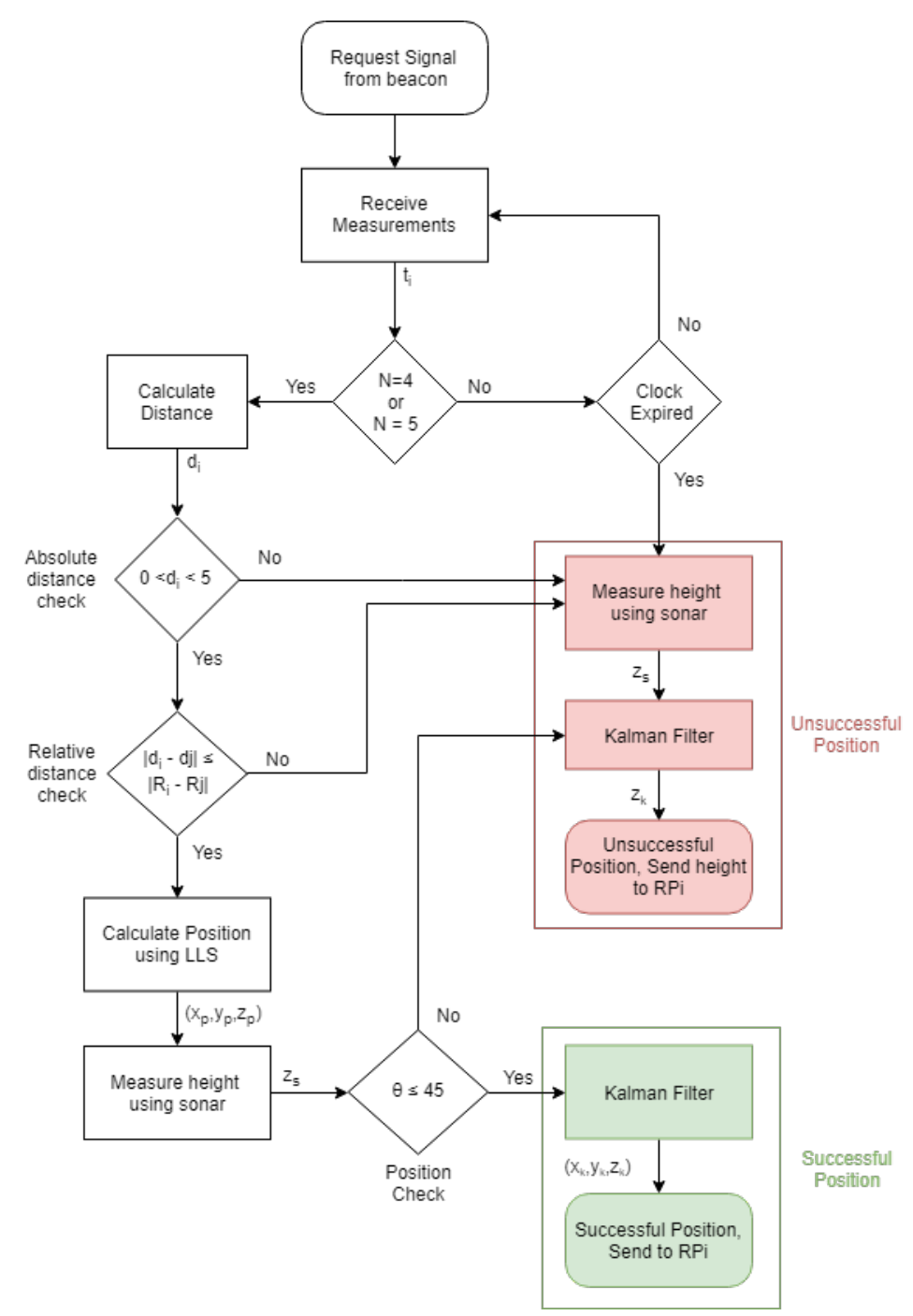

Figure 4.28: PSoC block diagram LLS

each frequency tested a similar number of successful measurements is obtained (approx. $70 \%$ ). Similarly, most frequencies tested also show a similar standard deviation around $5 \mathrm{~cm}$. However, at $1 \mathrm{~Hz}$ the standard deviation rises to over $20 \mathrm{~cm}$ due to a smaller sample size since each test is limited to $30 \mathrm{~s}$. This shows that an update rate greater than $5 \mathrm{~Hz}$ and up to $25 \mathrm{~Hz}$ does not have a significant impact on the results.

The maximum rate of the sonar is set to $10 \mathrm{~Hz}$ to limit interference between measurements [69]. Since lateral positioning of the quadrotor is more dynamic than 


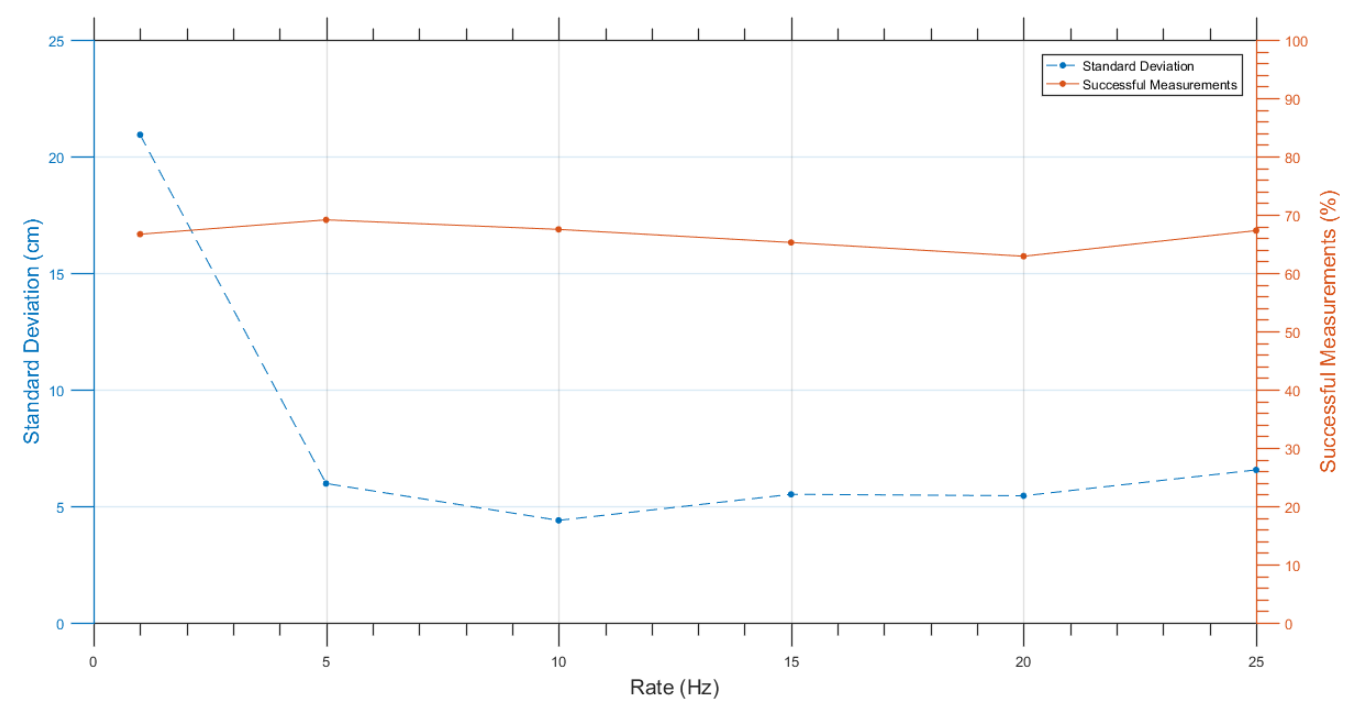

Figure 4.29: Transmission rate accuracy $\mathrm{P}(100,0,177)$

the altitude, it is desirable to have a higher update rate for the lateral position than the altitude. A nominal value of $10 \mathrm{~Hz}$ is selected for the lateral positioning rate because this is the nominal value for GPS update rate in ArduCopter [71]. To achieve this rate, the sonar operates at $2 \mathrm{~Hz}$, while the lateration system operates at $10 \mathrm{~Hz}$. The previous timestep height is used when the height is not measured. 


\section{Chapter 5}

\section{Results}

The results section begins with stationary system accuracy tests to determine the performance of the ULS using the different lateration algorithms. Next the system is implemented into the control scheme and tested in a GPS denied environment for hover. To improve the results a new algorithm is derived to enhance the accuracy. Using this algorithm TARA 3 completes a hover and a landing. The system is taken into an outdoor environment and the ULS positioning is compared against the raw GPS position. Finally the beacon travels in a line at a constant rate and the quadrotor tracks the beacon and then tries to land on the moving beacon.

Further stationary accuracy testing using configurations which are not used in flight are shown in Appendix A. Appendix B compares the performance of the ULS with the implemented control scheme and using GPS with ArduCopter's flight modes (land, GPS hold) in an outdoor environment for hover, landing, and completing a mission. 


\subsection{Lateration Algorithm}

\subsubsection{Accuracy Assessment}

The accuracy of the position system is evaluated by mounting TARA 3 at a fixed height of $177 \mathrm{~cm}$ and moving the beacon to various $(\mathrm{x}, \mathrm{y})$ locations on a positioning paper as shown in Fig. 5.1. Data is collected at a specific location for 30 seconds allowing for approximately 300 data points to be collected at each location. The axes are located on TARA 3 as this is how the position is calculated and the beacon moves relative to TARA 3 which is held stationary. The center position $(0,0)$ is aligned by using a drop string from the center of TARA 3. The positioning paper axes are aligned visually with the TARA 3 axes. For these tests the motors are running at half throttle such that TARA 3 is still suspended to simulate in flight vibrations and turbulence.

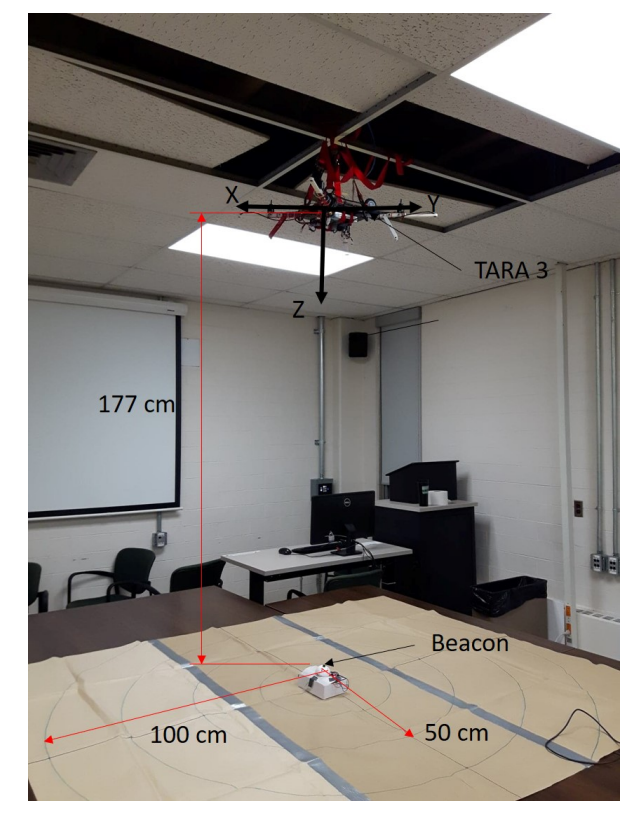

Figure 5.1: Accuracy testing

Different lateration algorithms are used depending on the number of receivers. As the number of receivers increase the expected accuracy is expected to increase 
because the algorithm accounts for more measurement error. However, the number

of successful measurements is expected to decrease because it requires more signals to be captured.

The results for the trilateration algorithm are shown in Fig. 5.2, this algorithm uses three corner receivers (receivers 2, 3, and 4) as shown with circles and are labelled on the quadrotor schematic. The arrow shows the forward direction of the quadrotor (where $+\mathrm{X}$ is forward). This figure follows a similar same format as presented in the dilution of precision section (Fig. 4.26). The nominal locations are located with solid circles and the mean of the position measurement is shown with an $\mathrm{X}$ marker. The dotted circles around each location represent the measured standard deviation at that location calculated as,

$$
\sigma_{x y}=\sqrt{\sum_{i=1}^{i=300} \frac{\left(x_{i}-\bar{x}\right)^{2}}{300}+\frac{\left(y_{i}-\bar{y}\right)^{2}}{300}}
$$

Where $\sigma_{x y}$ is the combined xy standard deviation represented by the dotted circle, and $\bar{x}$ and $\bar{y}$ represent the mean positions. The summation is over the 300 data points, and each individual measurement is denoted by $x_{i}$ and $y_{i}$. The written percentage values are the percentage of successful measurements at that location as determined by the algorithm (Fig. 4.27). In addition the RMSE is calculated at each location as,

$$
R M S E_{x y}=\sqrt{\sum_{i=1}^{i=300} \frac{\left(x_{i}-\hat{x}\right)^{2}}{300}+\frac{\left(y_{i}-\hat{y}\right)^{2}}{300}}
$$

Where $\hat{x}$ and $\hat{y}$ represent the desired positions as shown with solid circles. The average RMSE is shown in the top right corner along with the average standard deviation and the mean successful measurements. The standard deviation is the smallest near the centre and on average increases with the radius. Similarly, the number of successful measurements is greater near the centre and mostly decreases as the radius increases. The receivers located over the bottom right quadrant $(\mathrm{B}, \mathrm{E}, \mathrm{F}, \mathrm{I})$, 
are expected to have a better accuracy and more successful measurements since they are a closer proximity and decrease the propagation distance and beam angle $(\gamma)$. The average standard deviation is better for the bottom right quadrant (B,E,F,I) with $10.4 \mathrm{~cm}$ versus $12.3 \mathrm{~cm}$ for the top right quadrant (C,D,G,H). The average number of successful measurements is also better with $71 \%$ in the bottom right quadrant and $67 \%$ for the top left quadrant. The mean positions for the outer radius $(100 \mathrm{~cm})$ are all inside the expected radius. The ToF software delay is calibrated to be directly over the beacon which results in a better average position for the $50 \mathrm{~cm}$ radius and directly over the beacon. As the beacon moves outward the calculated position may be closer than actual.

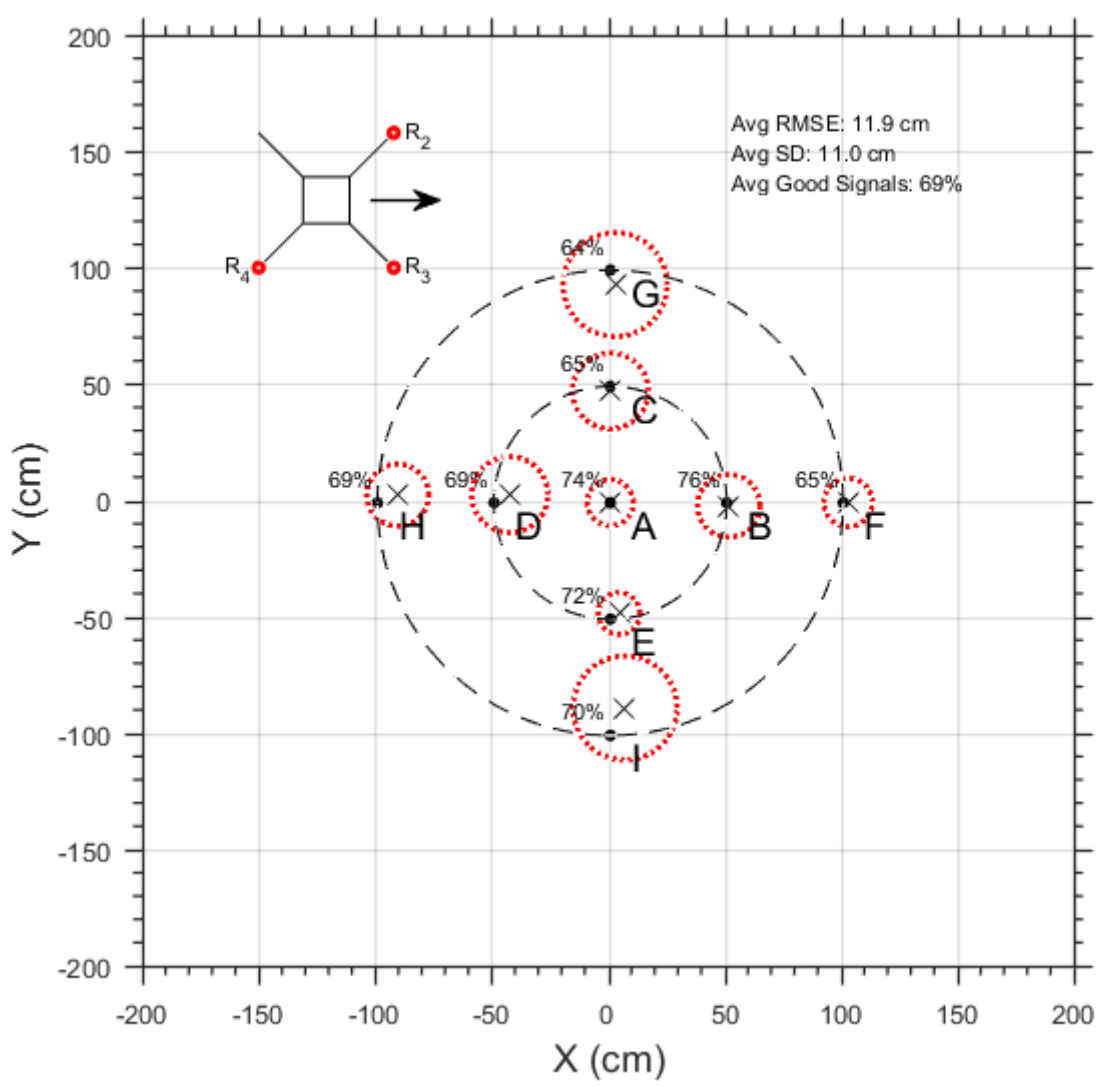

Figure 5.2: Trilateration position accuracy 
The results for the four-receiver algorithm are shown in Fig. 5.3 using the four corner receivers. It follows similar trends as the trilateration algorithm but has a large standard deviation at locations $\mathrm{E}$ and $\mathrm{G}$, this is attributed to incorrect distance measurements passing the measurement filter and contributing to incorrect position measurements leading to a high standard deviation. There is significant variation between the number of successful measurements with $77 \%$ in the middle and only $44 \%$ at point $\mathrm{G}$. The three-receiver algorithm slightly outperforms the four-receiver LLS algorithm in the average RMSE (11.9 vs $12.1 \mathrm{~cm})$ the average standard deviation $(11.0 \mathrm{~cm}$ vs $11.3 \mathrm{~cm})$ and average number of successful measurements (69\% vs 67\%). As the number of receivers increase the expected accuracy is expected to increase because the algorithm is accounting for measurement error. The only locations where the trilateration outperforms the four receiver LLS is location E, F and G which is due to incorrect distance measurements making it through the measurement filter. The number of successful measurements is expected to decrease because it requires more signals to be captured.

The five-receiver algorithm is shown in Fig. 5.4, it follows the same trends as the previous algorithms. It outperforms the three and four receiver algorithms for average RMSE and average standard deviation which is expected because increasing the number of measurements should increase the accuracy. It also calculates fewer successful measurements which supports that increasing the number of measurements will decrease the number of successful measurements calculations. The mean values of the outer radius are much closer to the nominal points as compared to the trilateration algorithm which shows the inconsistencies with the mean position at the outer radius is more of an algorithm related issue. The points are all still within the circle which shows that a small change of calibration of the software delay could increase the accuracy of the mean position at the $100 \mathrm{~cm}$ radius but will decrease the accuracy of the mean position at the $50 \mathrm{~cm}$ radius. When these standard deviations are compared 


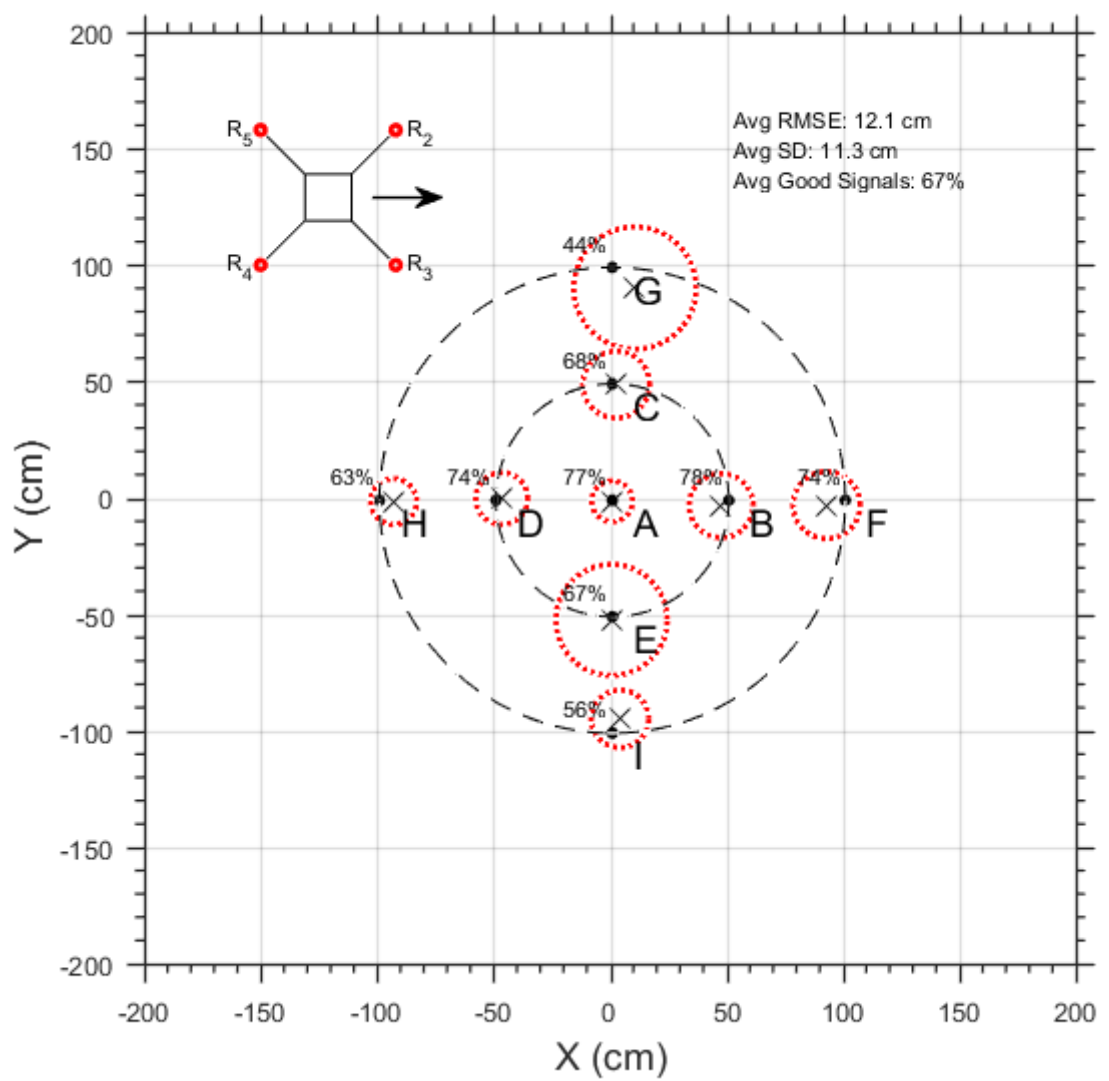

Figure 5.3: Four-receiver LLS position accuracy

with the HDoP values provided in Fig. 4.26, the actual position measurements have a lower standard deviation due to the measurement filters and Kalman filter which reduce noise.

The algorithms all have an average RMSE $<12.1 \mathrm{~cm}$, a standard deviation $<11.3$ cm but are limited by the number of successful measurements with all the algorithms less than $69 \%$. This can be converted to an average update rate of less than $6.9 \mathrm{~Hz}$. 


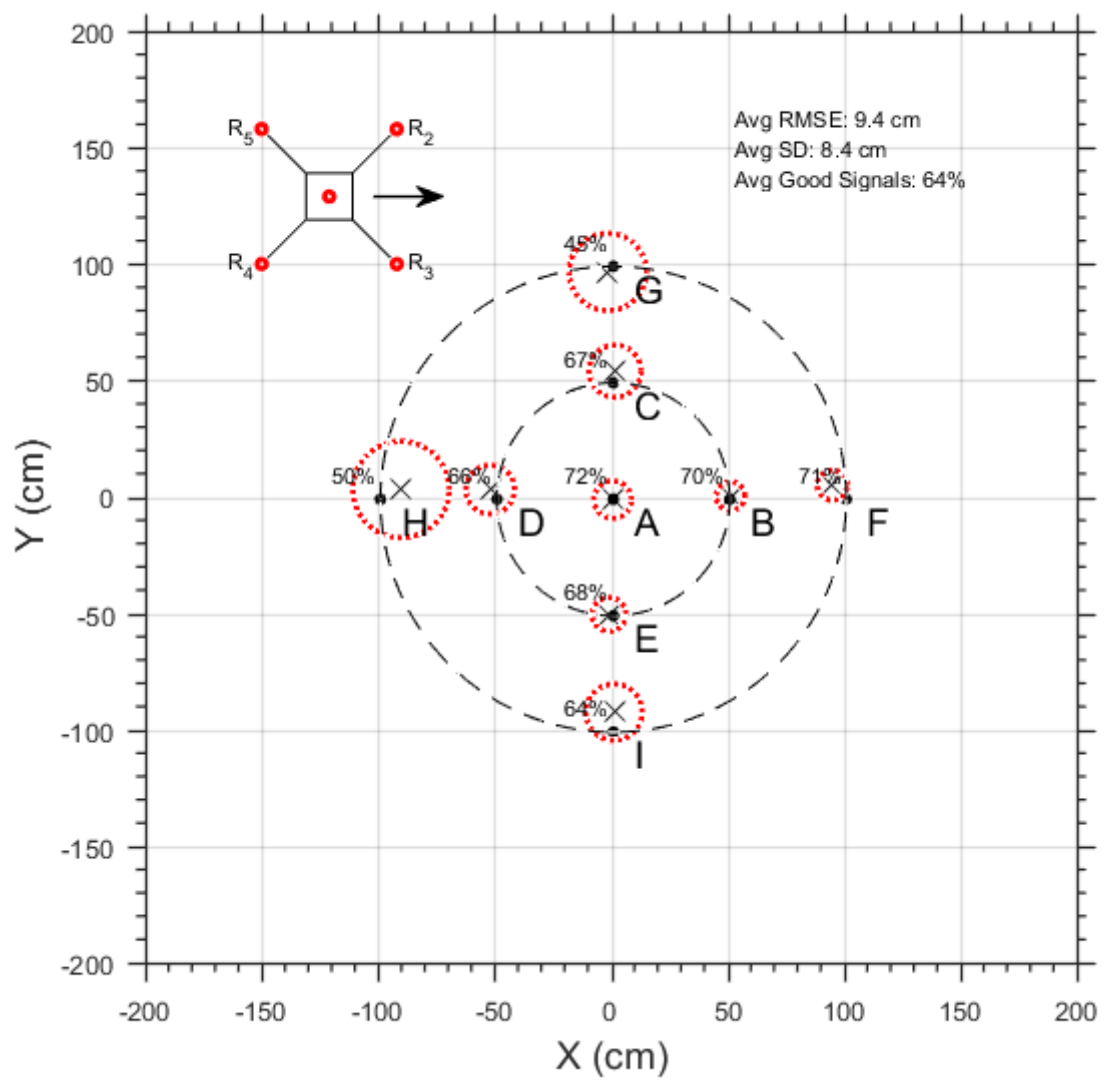

Figure 5.4: Five-receiver LLS position accuracy

\subsubsection{GPS-Denied Hover Flight Testing}

Flight tests are performed in a GPS denied environment located in a $9.75 \mathrm{~m}$ x $6.40 \mathrm{~m}$ x $5.64 \mathrm{~m}(\mathrm{~L} \times \mathrm{W} \times \mathrm{H})$ concrete walled and wooden floored room. In this environment there is no external wind but there are wind currents generated by the propellers. TARA 3 hovering over top of the beacon is shown in Fig. 5.5, where the beacon is labelled $\mathrm{C}(0,0,0)$ below TARA 3 . The coordinate axis have been rotated from centered on TARA 3 to centered on the beacon for visualization purposes since it is easier to understand. For each test TARA 3 is manually positioned above the beacon to an approximate position of $(0,-100,150)$ (located at point A) and set to autonomous 
mode as per section 3.2.3. In the first set of tests TARA 3 tries to position itself to location $\mathrm{B}(0,0,150)$ and then hover there using different lateration algorithms for comparison.

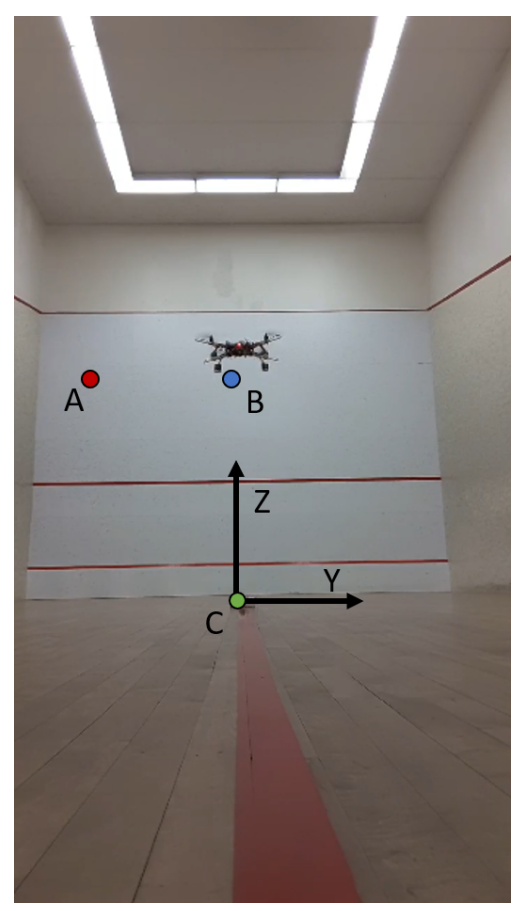

Figure 5.5: GPS-denied flight testing (X into the page)

Each test is run until TARA 3 drifts off and falls outside of the $45^{\circ}$ cone and can no longer localize itself. This typically occurs after 30 seconds so each test is only shown for the first 30 seconds in order to compare lateration algorithms for an equivalent time period. Before each test the ArduCopter is calibrated level to offset any IMU bias. Each test is shown for a hover of 30 seconds for comparison. The hover is started from an offset position $(0,-100,150)$ to require motion (essentially a step input).

The trilateration algorithm results are shown in Fig. 5.6. The position is displayed every time a successful position is calculated by the ULS as described in section 4.3. The algorithm uses receivers 2, 3, and 4, the same as the accuracy test in Fig. 5.2. 
The $\mathrm{X}$ position $\left(x_{k}\right)$ of TARA 3 is shown in the solid black line and the Y position $\left(y_{k}\right)$ of TARA 3 is shown as the dashed grey line. The RMS error is calculated between the current position and the desired position for each position calculated on each axis. This is used as relative comparison between tests. The good signals are calculated from the number of successful measurements divided by the total amount of requested signals. During the test TARA 3 overshoots the beacon in the Y direction before returning to the desired $\mathrm{y}=0$ position above the beacon. The $\mathrm{X}$ position is started above the beacon $(\mathrm{x}=0)$ and remains within $50 \mathrm{~cm}$ of the beacon. This algorithm has an RMS error of $38.1 \mathrm{~cm}$ for the $\mathrm{X}$ axis and $72.4 \mathrm{~cm}$ for the $\mathrm{Y}$ axis. This algorithm calculates a successful position $47 \%$ of the time which is lower than the stationary test (69\%). This is due to attitude changes which effect the beam angle and increased noise since the propellers are now running at hover throttle rather than half hover throttle. The average update rate is $4.7 \mathrm{~Hz}$.

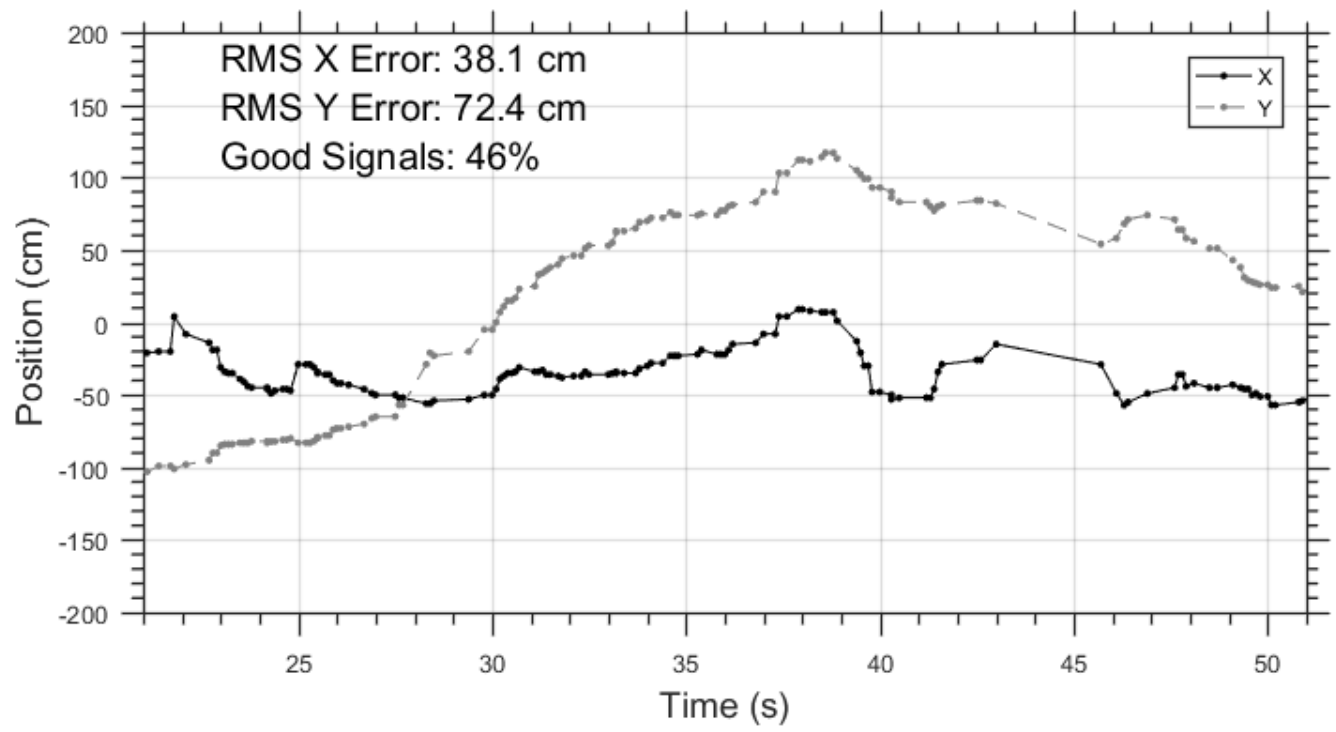

Figure 5.6: Hovering in GPS-denied environment using three-receiver algorithm

The results for the four-receiver LLS algorithm results are shown in Fig. 5.7. It uses the four corner receivers which is the same setup as the accuracy test in Fig. 
5.3. Along the $\mathrm{Y}$ axis it overshoots the beacon twice. Similar to the trilateration algorithm, it remains within $50 \mathrm{~cm}$ along the $\mathrm{X}$ axis. The RMS error is $29.2 \mathrm{~cm}$ for the $\mathrm{X}$ axis and $69.3 \mathrm{~cm}$ for the $\mathrm{Y}$ axis, which is $24 \%$ and $4 \%$ respectively better than the trilateration algorithm. This shows that it has better performance using the fourreceiver LLS algorithm in terms of RMSE. The number of successful measurements is $46 \%$ which is $1 \%$ less than the three receivers, this makes sense as more measurements are required. This update rate is less than the $67 \%$ in the stationary tests due to attitude changes and increased noise. The average update frequency is $4.6 \mathrm{~Hz}$.

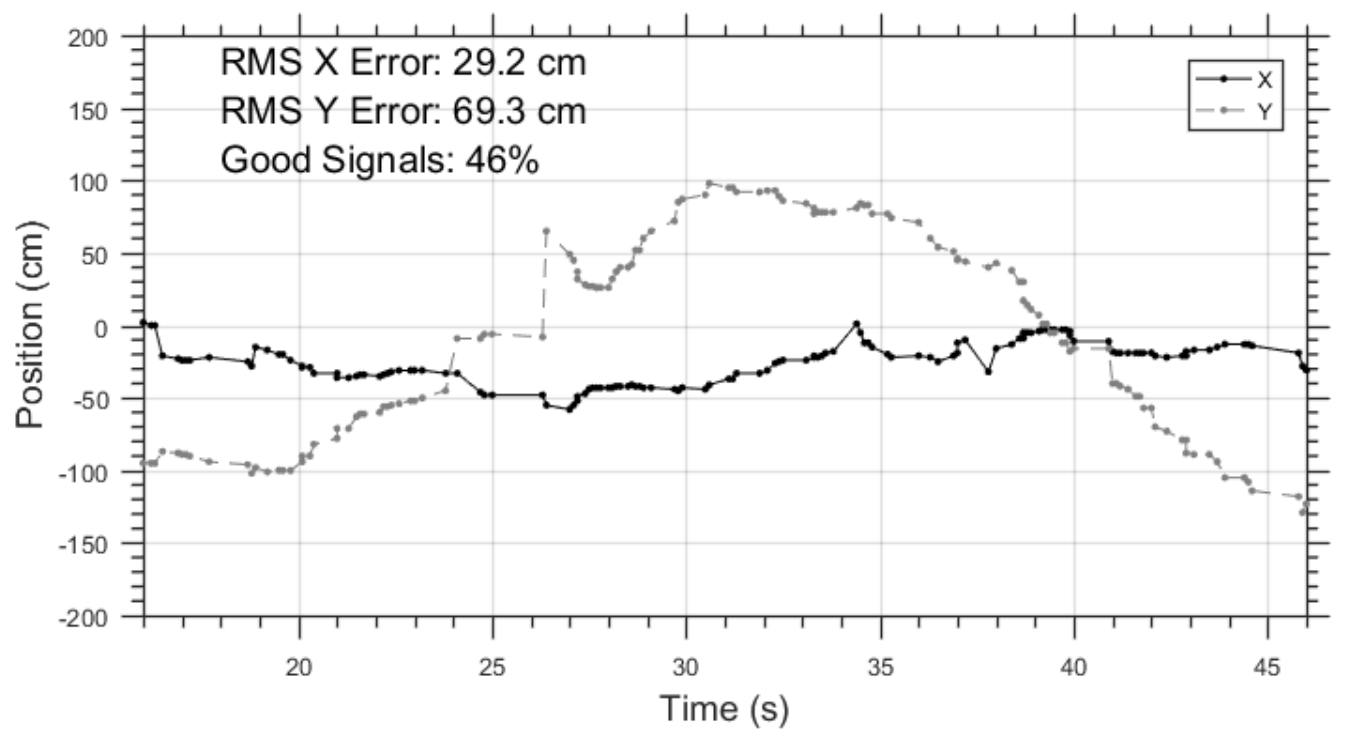

Figure 5.7: Hovering in GPS-denied environment using four-receiver algorithm

The five-receiver LLS algorithm results are shown in Fig. 5.8. Along the Y axis it starts with similar behavior, overshooting the beacon but then begins converging on the beacon near the end. Along the $\mathrm{X}$ axis, it remains within $50 \mathrm{~cm}$ of the beacon. The RMS error for the $\mathrm{X}$ axis is $27.7 \mathrm{~cm}$ and for the $\mathrm{Y}$ axis it is $57.2 \mathrm{~cm}$ which is $5.1 \%$ and $17.4 \%$ respectively better than the four-receiver LLS algorithm. This makes sense as it is a more accurate algorithm as shown in Fig. 5.4. The percentage of good signals decreased by $1 \%$ because it requires an extra successful measurement. The 
number of successful measurements is less than the stationary tests (64\%) due to the same reasons as the other algorithms. The average update frequency is $4.5 \mathrm{~Hz}$.

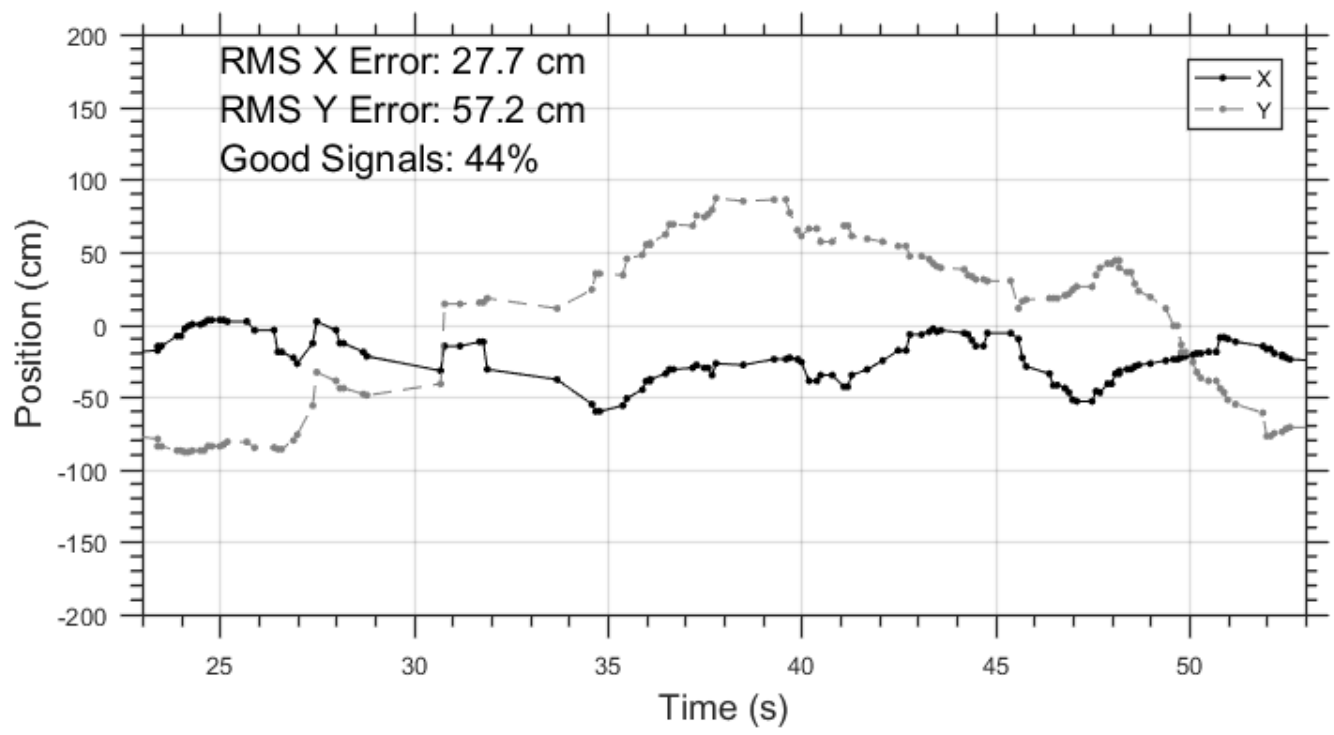

Figure 5.8: Hovering in GPS-denied environment using five-receiver algorithm

None of these algorithms truly converge to the beacon along the $\mathrm{Y}$ axis for an extended period time before drifting outside of the $45^{\circ}$ cone. Furthermore, the most accurate system only has $45 \%$ of the signals requested resulting in a successful position estimate. Therefore a new algorithm is proposed to perform localization.

\subsection{Combined Lateration Algorithm}

Localization can be performed if three or more succesful distance measurements are obtained. In the previous examples a successful position required all of the distance measurements to pass the measurement filters. To improve the number of successful positions, the two algorithms (Figs 4.27 and 4.28) are combined as shown in Fig. 5.9 to form the combined lateration algorithm. As the signals are received the distance measurement filters are applied. Any measurement that is not received or does not 
pass a filter is removed. If there are five distance measurements, LLS with all five measurements is used to obtain the best accuracy. If there are any four successful measurements LLS is still used for localization, this is not limited to the four corner receivers as previously discussed. If there are only three successful measurements trilateration is used. This can be any three distance measurements as discussed in Table 4.3 and not limited to only $\boldsymbol{R}_{2}, \boldsymbol{R}_{3}$, and $\boldsymbol{R}_{4}$ as the previous trilateration algorithm. If less than three successful distance measurements are obtained an unsuccessful position is obtained. 


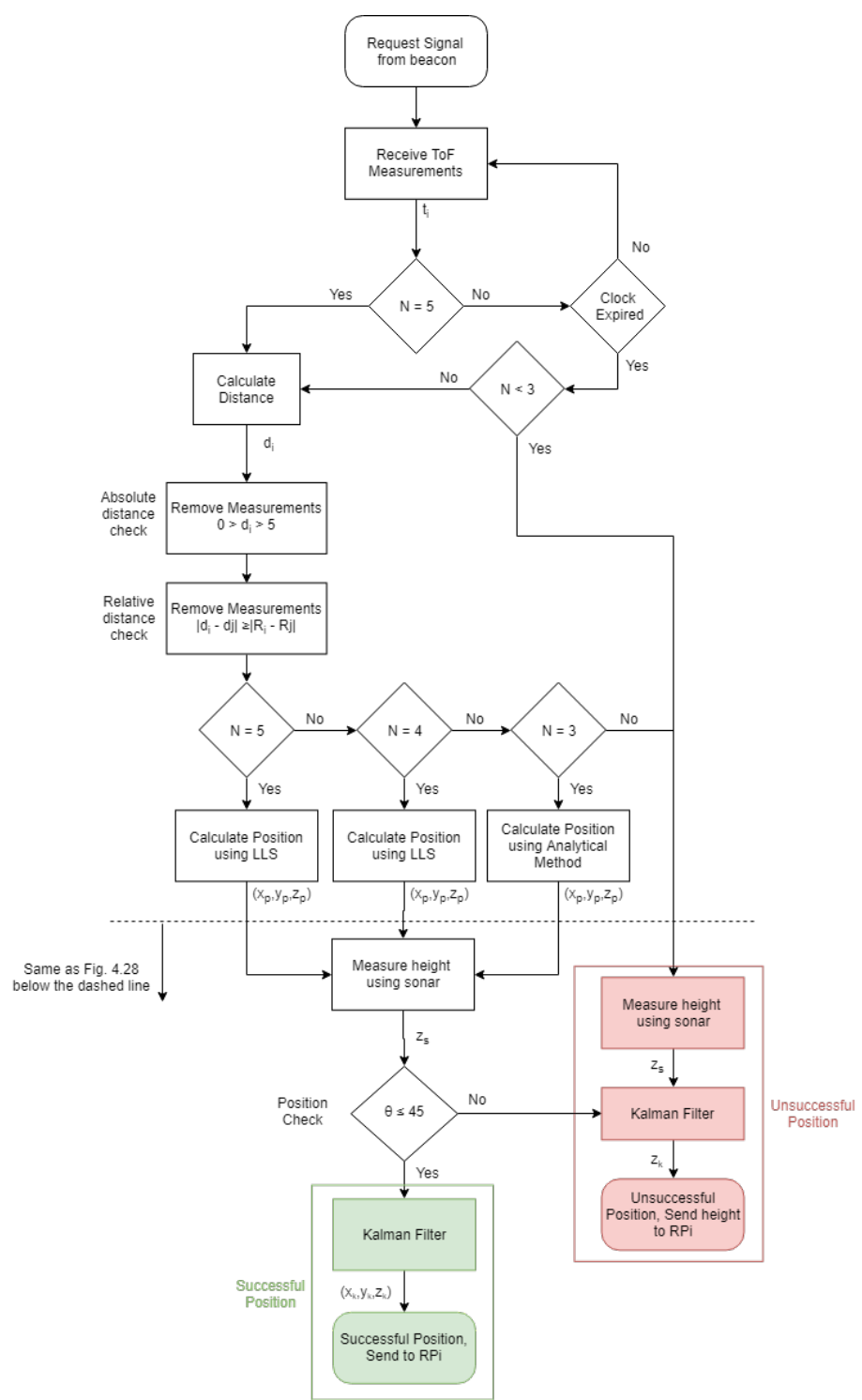

Figure 5.9: PSoC block diagram combined lateration algorithm 


\subsubsection{Accuracy Assessment}

The combined lateration algorithm position accuracy is shown in Fig. 5.10. The mean positions are closer to the nominal locations than the previous algorithms with the outer radius mean positions only slightly inside the radius. This has a 7\% (8.7 vs $9.4 \mathrm{~cm}$ ) better RMSE, a 5\% (8.0 vs $8.3 \mathrm{~cm}$ ) better standard deviation and 29\% (93\% vs 64\%) more successful measurements than the five-receiver LLS algorithm. The average update rate is $9.3 \mathrm{~Hz}$. The better accuracy can be attributed to calculating a successful position more often. This helps the Kalman filter remove more noise since the time steps between ULS position updates are smaller. The multiple receiver algorithm provides more consistency from the different beacon locations.

Comparing this with other ultrasonic literature with applications to flying, Eckert reports a maximum position error of $2 \mathrm{~cm}$ and Paredes [40] has a standard deviation of $3.7 \mathrm{~cm}$ with a $2.4 \mathrm{~Hz}$ update rate. The system is less accurate than these other two systems but the receivers are mounted on the quadrotor which enables the portable system. This system has a higher update rate than Paredes system, Eckert does not list the update rate. Comparing this accuracy to the accuracy of the AT\&T system with the same ultrasonic configuration. The AT\&T configuration has a $9 \mathrm{~cm}$ accuracy (95\% confidence) and the combined lateration algorithm has an accuracy of $15.7 \mathrm{~cm}$ ( $95 \%$ confidence) which is $74 \%$ worse. However, this is acceptable and makes sense due to the geometry of the receivers, in this application they are located on the drone which are relatively close which leads to a high DoP. In the AT\&T application the receivers are located in a room around the beacon which leads to a lower DoP. 


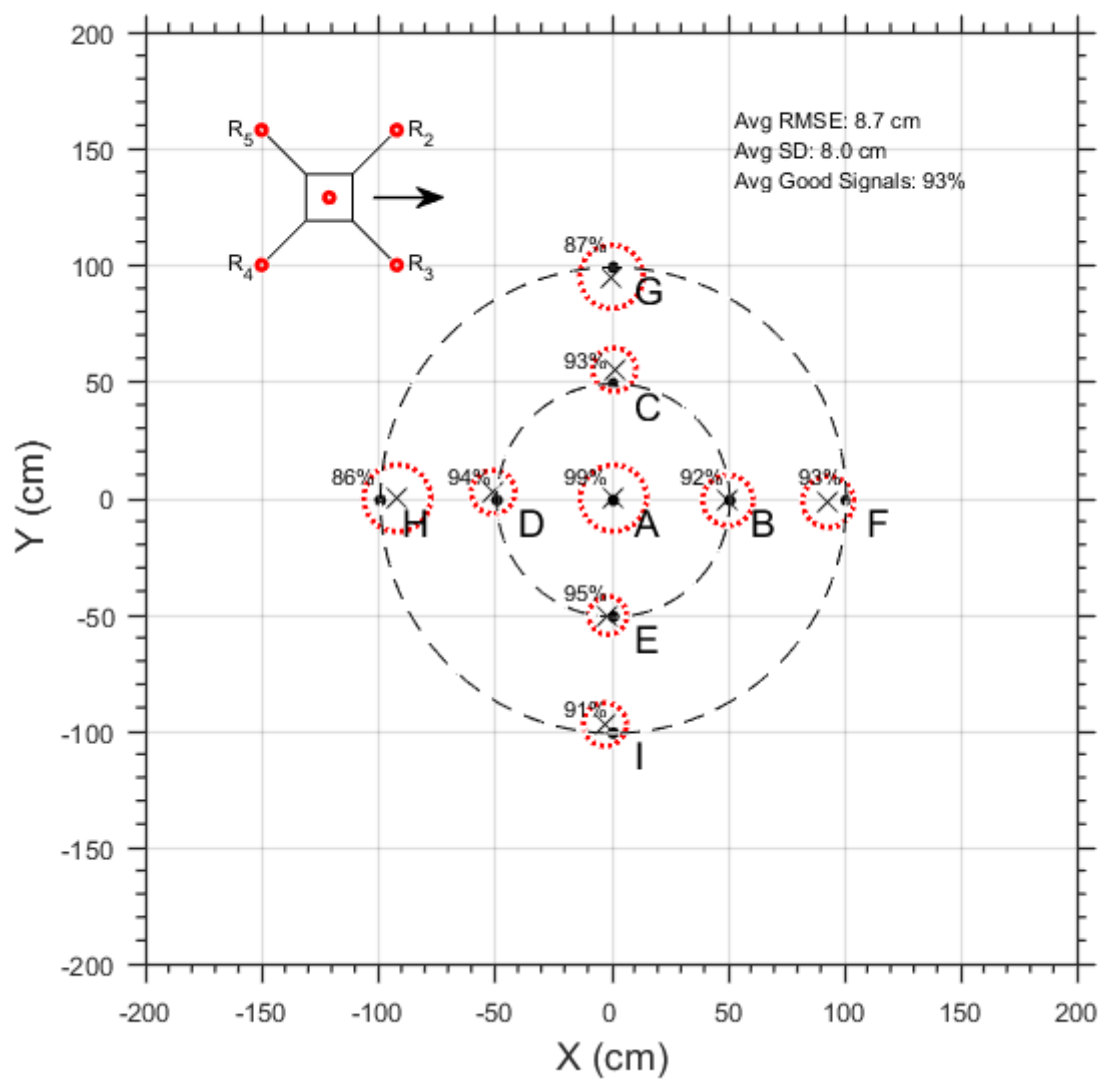

Figure 5.10: Combined lateration position accuracy

\subsubsection{GPS-Denied Hover Flight Testing}

This algorithm is tested at the same GPS-denied location as the previous lateration algorithms. The combined lateration algorithm results are shown in Fig. 5.11. Initially along the Y axis TARA 3 overshoots the beacon as it seeks to reduce the initial offset of $\mathrm{y}=-100 \mathrm{~cm}$ but does well to converge towards $\mathrm{y}=0$ afterwards. Similarly, as the previous tests, TARA 3 remains within $50 \mathrm{~cm}$ along the $\mathrm{X}$ axis. The RMS error for the $\mathrm{X}$ axis is $28.7 \mathrm{~cm}$ and $32.7 \mathrm{~cm}$ for the $\mathrm{Y}$ axis, the $\mathrm{X}$ axis performed $3 \%$ (28.7 vs $27.7 \mathrm{~cm}$ ) worse than the five receiver algorithm but this error is still within half a wingspan. For the $\mathrm{Y}$ axis the RMS error is 32.7 which is $43 \%$ (32.7 vs $57.2 \mathrm{~cm}$ ) 
better than the five-receiver LLS algorithm. The number of successful measurements has increased to $81 \%$ which is an increase of $35 \%$ ( $81 \%$ vs $46 \%$ ) over the trilateration algorithm. The successful measurements percentage is less than the stationary performance (93\%) due to increased noise and a changing attitude. The average update frequency is $8.1 \mathrm{~Hz}$. This algorithm proves to be superior in terms of accuracy and update frequency than the previous algorithms.

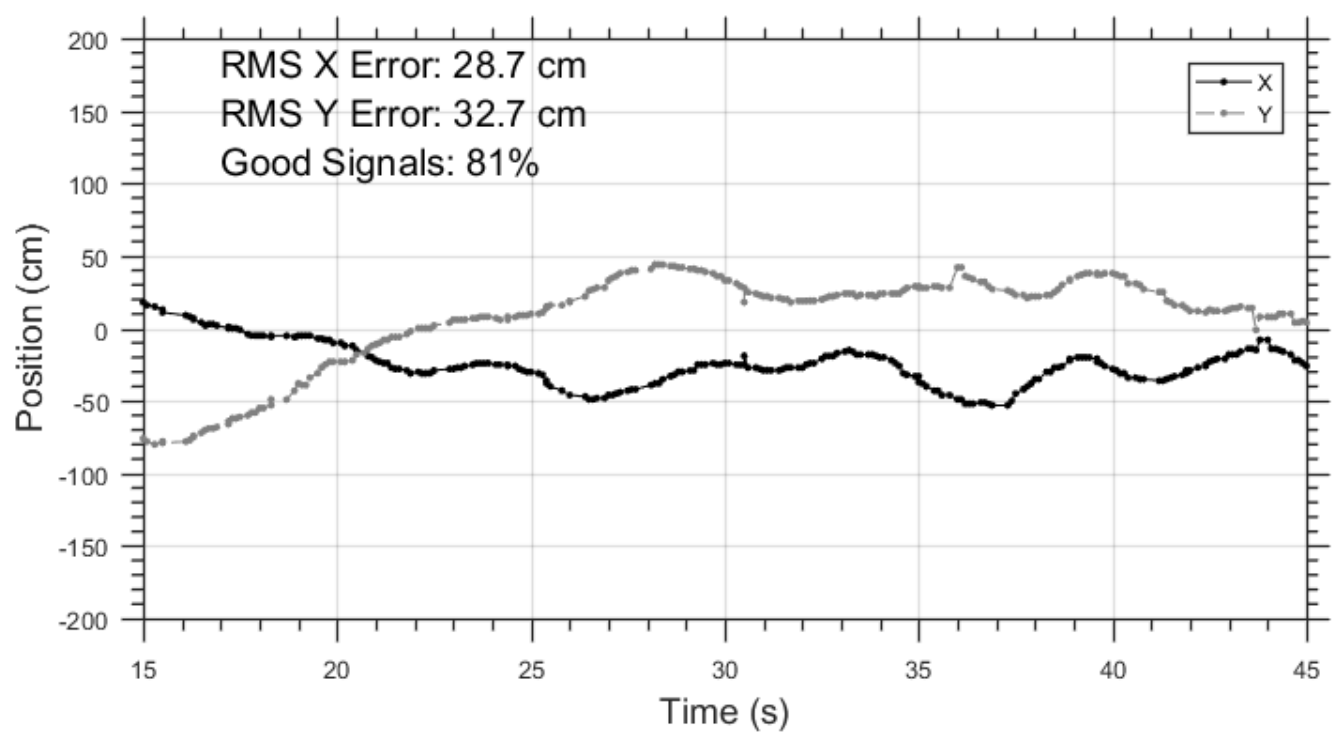

Figure 5.11: Hovering in GPS-denied environment using combined lateration algorithm

An extended hover using the multiple receiver algorithm lasting nearly two minutes is shown in Fig. 5.12. The position converges with an RMS error of $23.8 \mathrm{~cm}$ for the $\mathrm{X}$ axis and $40.6 \mathrm{~cm}$ for the $\mathrm{Y}$ axis. For the majority of the flight TARA 3 stays within a half wingspan $(40 \mathrm{~cm})$ over the beacon, with an average position update rate of $8.3 \mathrm{~Hz}$. The altitude control is shown in Fig 5.13 where TARA 3 is shown to hover near the desired height of $1.5 \mathrm{~m}$ (shown in grey), with an average error of $7.5 \mathrm{~cm}$ or within $1 \%$ of the desired. 


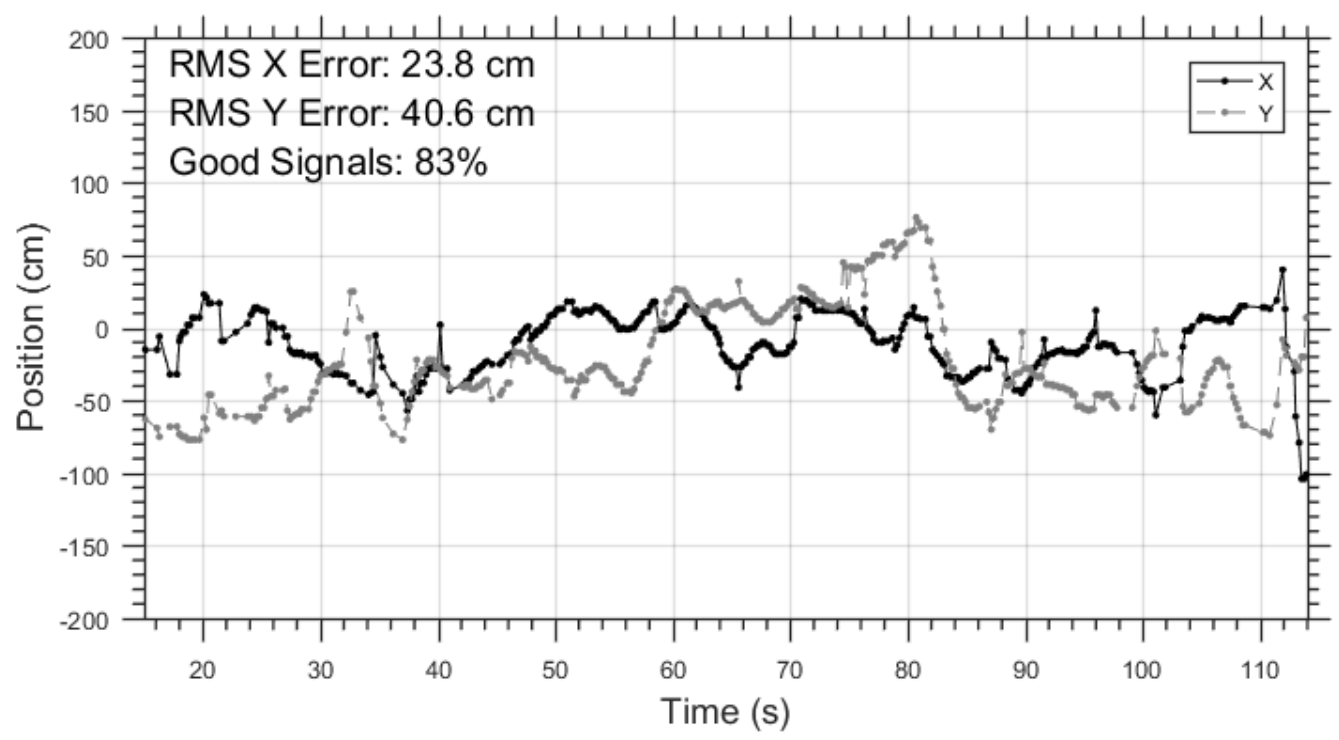

Figure 5.12: Extended hovering in GPS-denied environment using combined lateration algorithm

\subsubsection{GPS-Denied Landing}

For the landing data presented, the logic is presented in Fig. 5.14. From a hovering state the quadcopter will try to position itself over the beacon. If it is within a half wingspan radius $(40 \mathrm{~cm})$ it will begin to descend (center cylinder Fig. 5.15) at a rate of $50 \mathrm{~cm} / \mathrm{s}$ while still controlling the position to directly over the beacon, $(0,0)$. If TARA 3 leaves the acceptable radius the descent will stop and it will correct the position back toward $(0,0)$ while holding altitude. TARA 3 will continue to descend while remaining within the acceptable radius using this algorithm until it reaches a minimum height, $80 \mathrm{~cm}$. Below this height the receivers cannot properly compute positions due to the beam pattern. Therefore, once this height is reached TARA 3 begins a steady descent until reaching the ground at a rate of $50 \mathrm{~cm} / \mathrm{s}$. Below a height of $30 \mathrm{~cm}$ the downward facing sonar stops receiving accurate data and thus the autopilot relies on inertial measurements until touchdown.

For the autonomous landing, TARA 3 starts from approximately point A in Fig. 


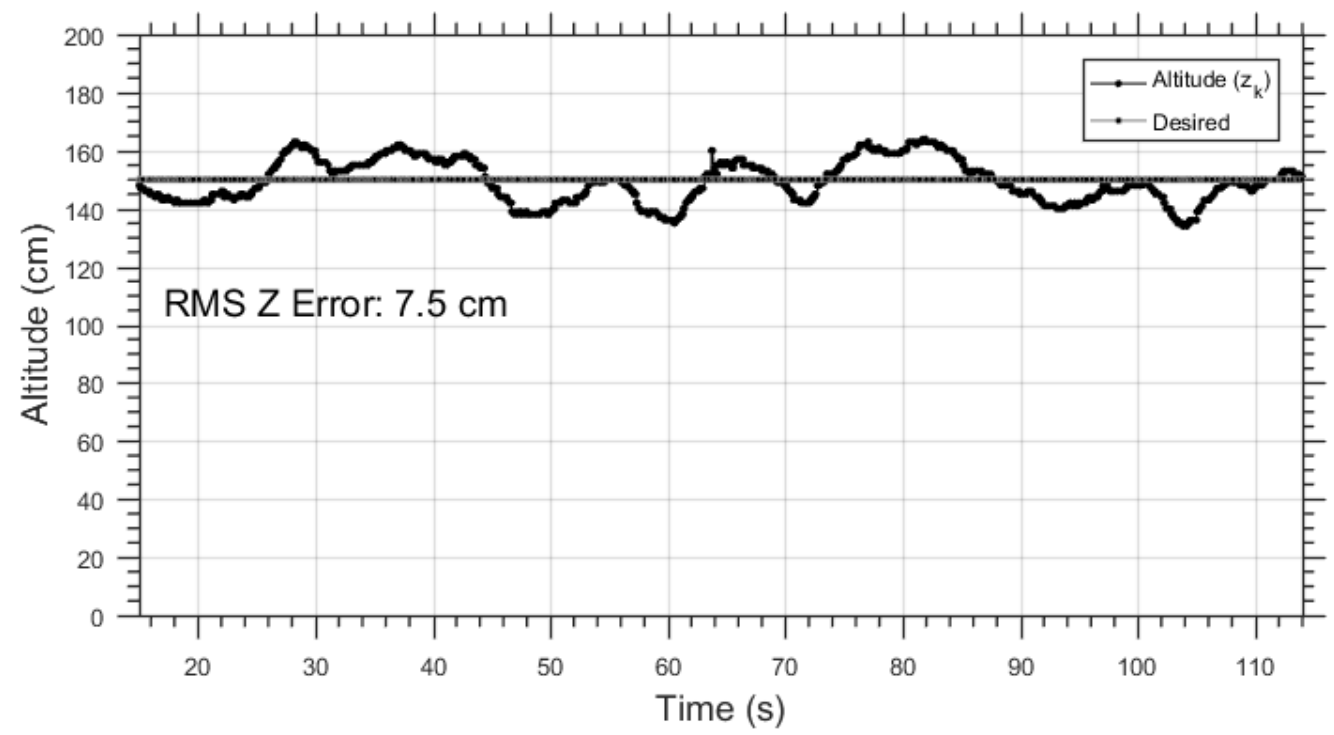

Figure 5.13: Extended hovering altitude using combined lateration algorithm

$5.5(0,-100,150)$ and attempts to land on the beacon at point C. TARA 3 is positioned manually to point A and set to autonomous mode as in previous flight tests. Figure 5.16 shows the autonomous landing results where the figure starts after TARA 3 has been switched to autonomous mode (at $13 \mathrm{~s}$ ). The top figure shows the $\mathrm{X}$ position with a solid black line and the $\mathrm{Y}$ position as a dashed grey line against time. The bottom figure shows the height of TARA 3 against time. As TARA 3 moves from the initial offset along $\mathrm{Y}$ to directly over the beacon it is trying to maintain a hover at $150 \mathrm{~cm}$ as shown by the line $<19 \mathrm{~s}$. The acceptable radius lines to start the landing procedure are shown as dotted lines in the XY position graph, then at $19.5 \mathrm{~s}$ TARA 3 is within the acceptable radius and thus begins to descend. For the remainder of the flight TARA 3 remains within the acceptable radius as shown with the large shaded region. A dashed dot line indicates the final descent height $(80 \mathrm{~cm})$ which TARA 3 reaches at 24 seconds and thus continues to descend regardless of position as shown with the smaller shaded region. The dashed double dot line shows the final height which the sonar can detect which is reached just before 28 seconds. The final distance 


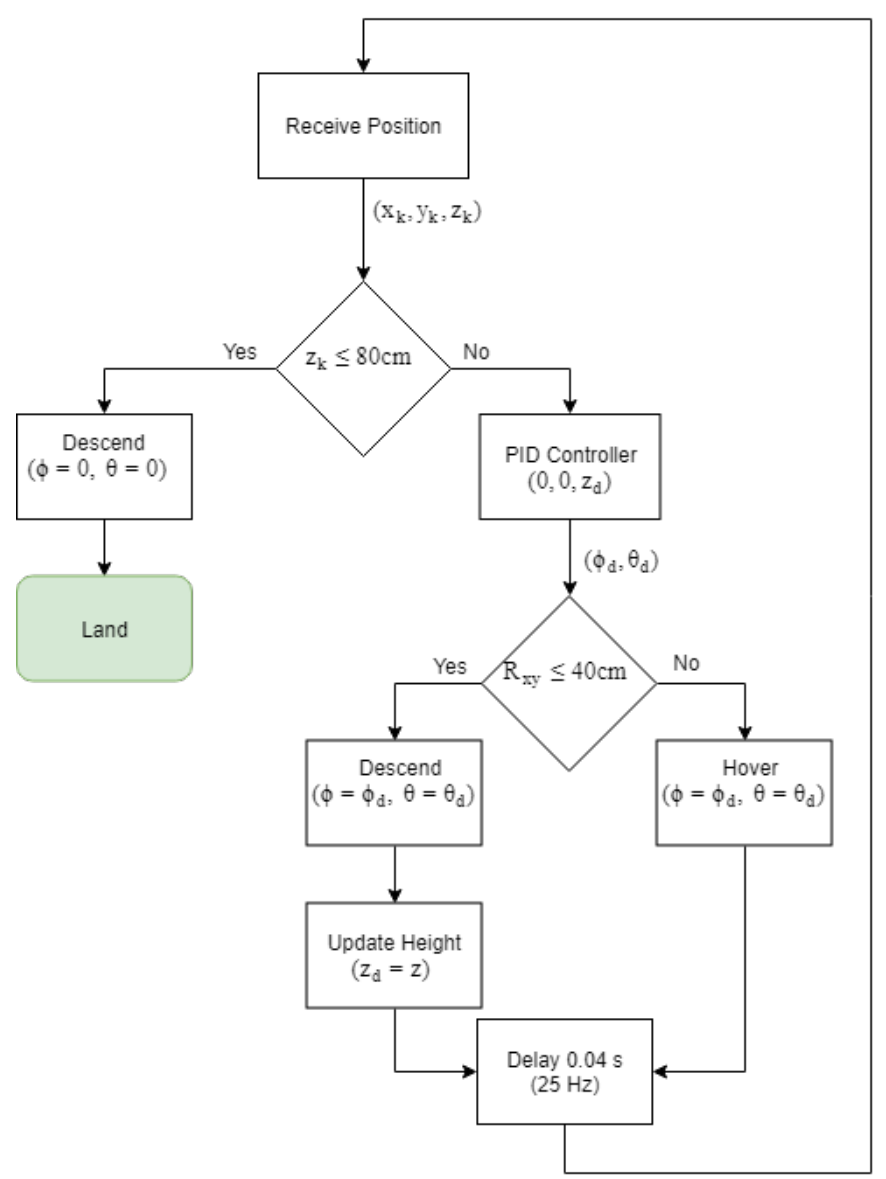

Figure 5.14: Landing flow diagram

from the beacon to the center of the quadrotor is measured to be $18 \mathrm{~cm}$ or $22.5 \%$ of the wingspan. The landing took a total of $15.5 \mathrm{~s}$ from the time TARA 3 is switched to autonomous mode at a position of $(0,-100,150)$ to landing. 


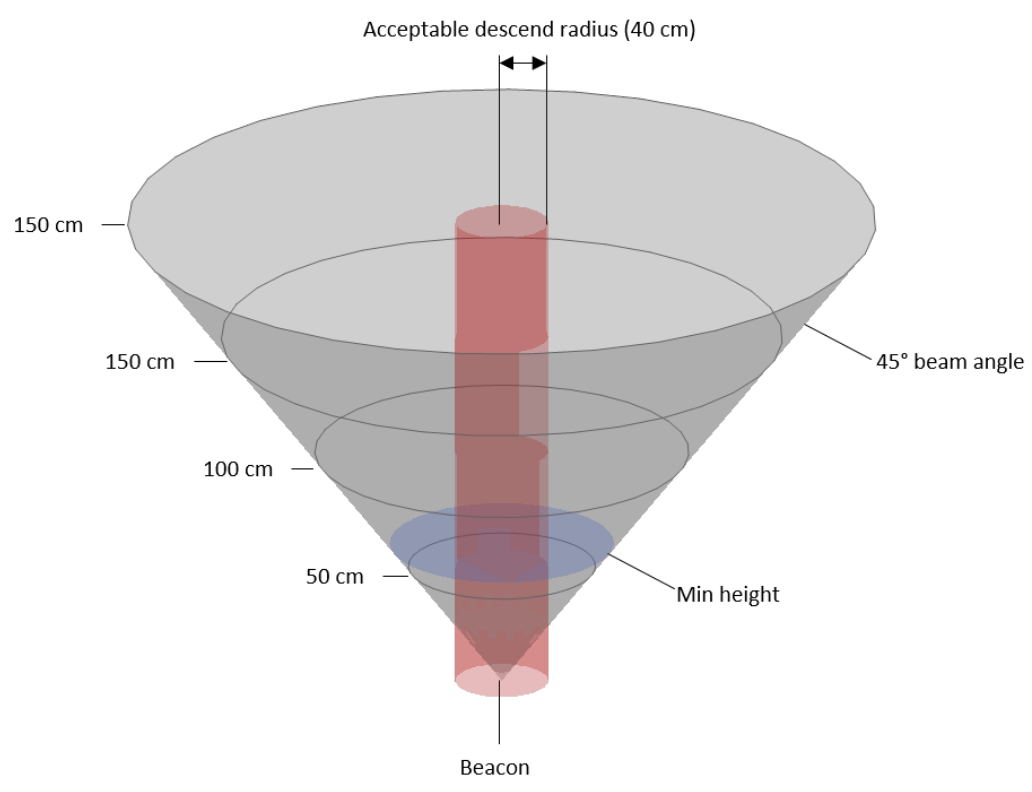

Figure 5.15: Landing visualization

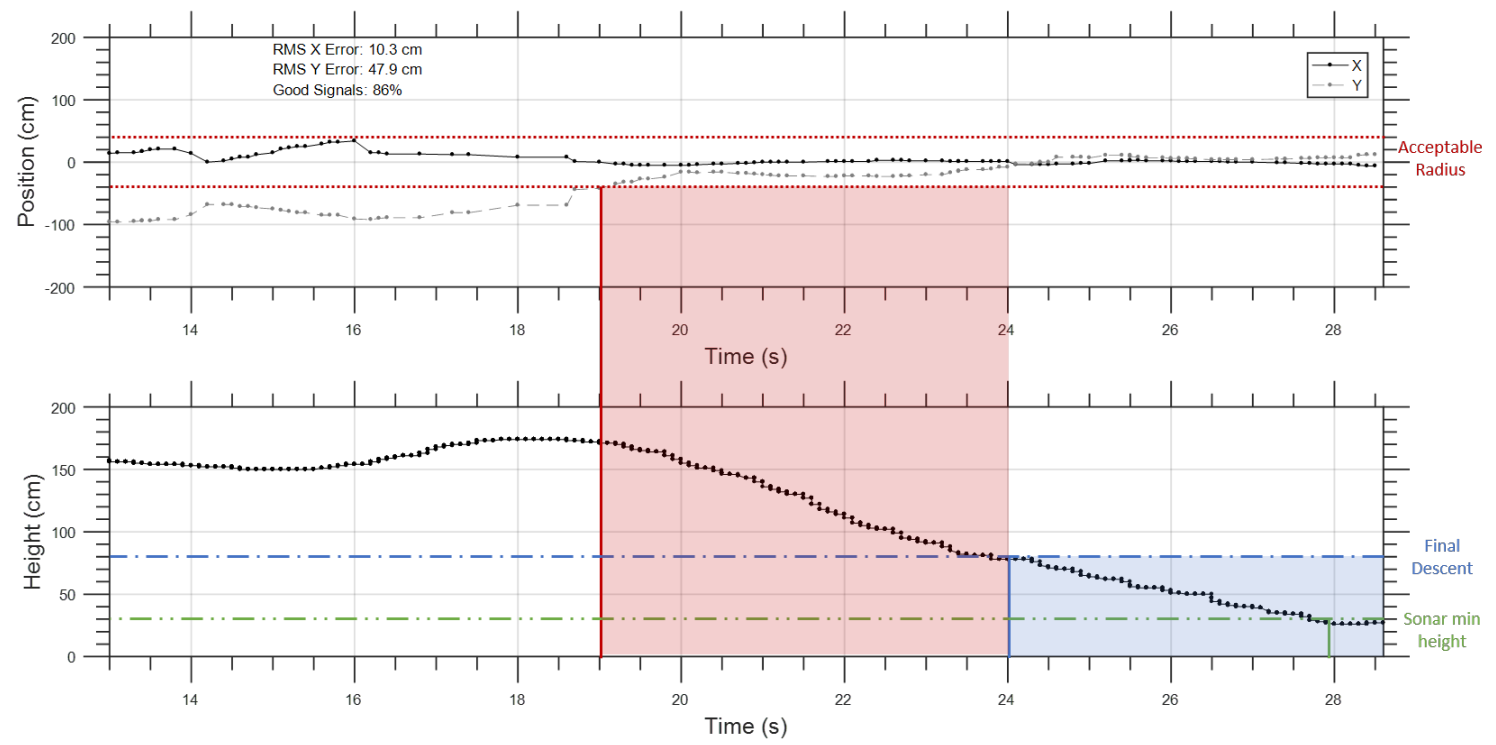

Figure 5.16: Landing in GPS-denied environment 


\subsection{GPS Comparison}

To compare the performance of the ULS to a raw GPS signal outdoor tests are conducted on private property in Class E airspace outside Carleton Place, Ontario on August 24 2019. The drone has een registered with Transport Canada under C1926038730. Figure 5.17 shows TARA 3 hovering over the beacon, shown in white. This location is chosen because it is isolated from people to create a controlled environment. The coordinate axes are shown on the beacon, the axes are flipped from TARA 3 to the beacon for visualization purposes. The location is moved from the picture to a different location on the property to allow for flight testing over smooth pavement. Conducting the tests over grass caused the downward facing sonar to be noisy. A Bios home weather station (Product BW995) weather vane is shown on the right, this is to view any gusts which occur during video review. This data is recorded on a day with minimal wind with an average wind speed of $0.0 \mathrm{~km} / \mathrm{hr}$, a temperature of $22.4^{\circ} \mathrm{C}$, an atmospheric pressure of $99327 \mathrm{~Pa}$ and a relative humidity of $61 \%$. The data is sampled once every two minutes thus it does not give insight into wind gusts.

\subsubsection{Stationary Testing}

The accuracy of the setup is tested in an open environment to compare to the reported accuracy. The GPS is mounted to the quadrotor and left in a fixed position on the ground for five minutes. The reported accuracy is provided in Table 5.1, the reported parameters are also included again for convenience. The measured frequency is $70 \%$ lower than the reported maximum frequency. The velocity and heading accuracy are not measured experimentally.

The combined lateration algorithm has a $1040 \%$ (8.0 vs $91.1 \mathrm{~cm})$ better average standard deviation than the GPS. The ULS also has a 56\% (9.3 vs $4.1 \mathrm{~Hz}$ ) better update rate than the raw GPS signal. 


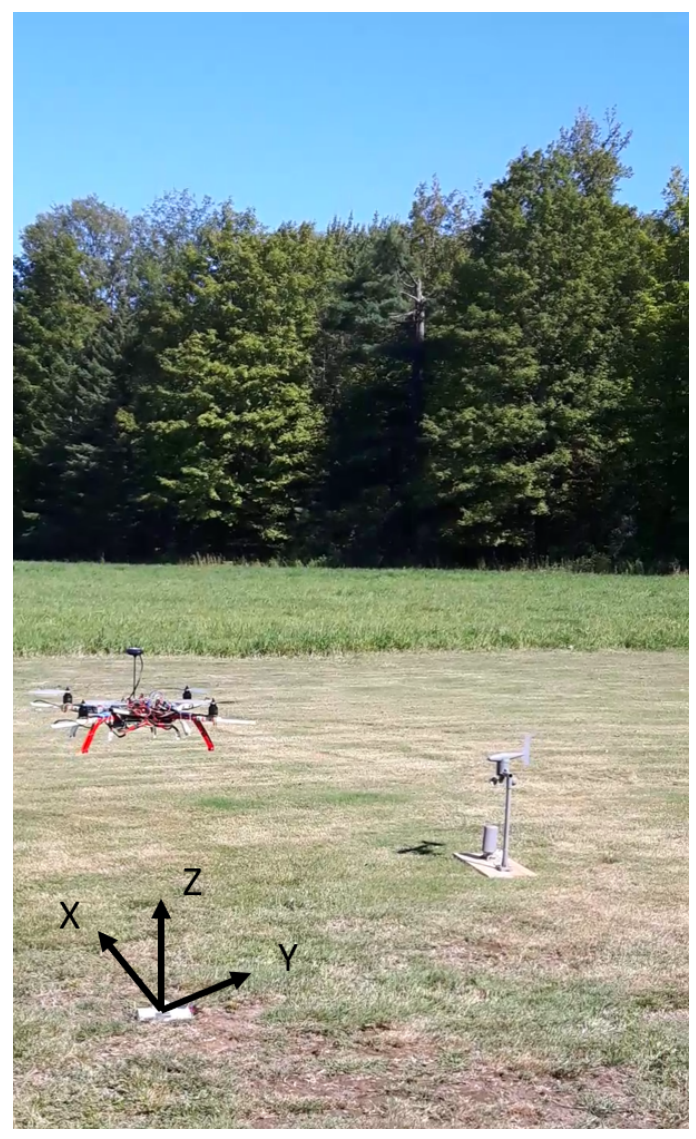

Figure 5.17: Outdoor flight-testing using TARA 3

\subsubsection{Hover Testing}

To test the relative accuracy of the systems TARA 3 conducts an ULS controlled hover directly above the beacon to a desired position of $(0,0,250)$. The raw GPS signal is measured during the hover but is not used for control. The GPS location has been calibrated to the beacon such that the origin $(0,0,0)$ for each system are the same. The $\mathrm{X}$ axis data is shown in Fig. 5.18 where the black solid line is the ULS $\mathrm{X}$ position $\left(x_{k}\right)$ and the grey dashed line is the raw GPS position $\left(x_{G}\right)$. TARA 3 is manually positioned above the beacon and just after $16 \mathrm{~s}$ is switched to autonomous hover (where the figure begins). The raw GPS signal has significantly more noise than the ULS with many sharp peaks. Over time the GPS position begins to drift 
Table 5.1: GPS Accuracy

\begin{tabular}{|c|c|c|c|}
\hline Parameter & Reported [63] & Experimental & Units \\
\hline \hline Accuracy & 250 (Absolute) & $91.1(\mathrm{SD})$ & $\mathrm{cm}$ \\
\hline Frequency & $<14$ & 4.1 & $\mathrm{~Hz}$ \\
\hline Velocity Accuracy & 5 & N/A & $\mathrm{cm} / \mathrm{s}$ \\
\hline Heading Accuracy & $0.3^{\circ}$ & N/A & N/A \\
\hline
\end{tabular}

with the first half of the hover having a smaller difference between the second half. The test is manually stopped after $112 \mathrm{~s}$ due to low battery concerns. The average error is calculated as the difference between the ULS signal and the raw GPS signal, there is an average difference of $-23.0 \mathrm{~cm}$. The average ULS update rate is $9.3 \mathrm{~Hz}$ which is $132 \%$ faster than the $4.0 \mathrm{~Hz}$ average update rate of the GPS. The Y axis is shown in Fig. 5.19 which shows similar behaviour as the $\mathrm{X}$ axis with an average error of $-23.1 \mathrm{~cm}$. Both positions start near $\mathrm{y}=0$ but as the test progresses the GPS data drifts further away from the ULS data. The $\mathrm{Z}$ axis is shown in Fig. 5.20, with the desired altitude shown with a dash dot line at $250 \mathrm{~cm}$. The ULS measures the height as initially below $250 \mathrm{~cm}$ and controls up to the desired height. The GPS height fluctuates above and below the ULS height with an average variation of 34.5 $\mathrm{cm}$ from the desired height of $250 \mathrm{~cm}$.

This test shows that the ULS has superior performance than the raw GPS position data during flight tests while hovering over the beacon. The ULS has a better update rate and does not drift as the GPS data has shown. 


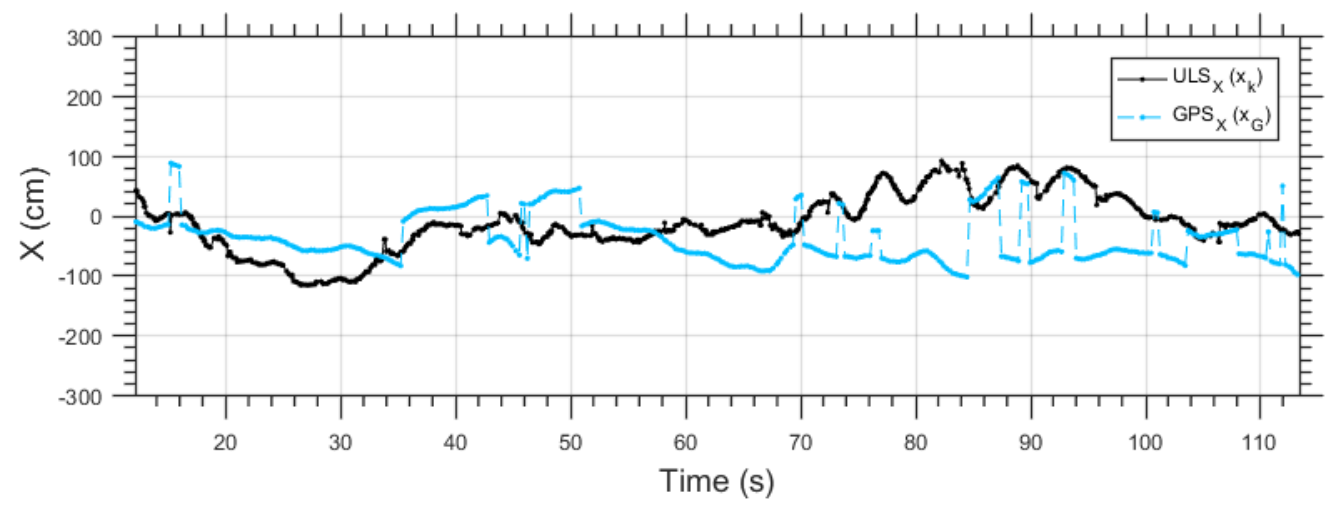

Figure 5.18: ULS - GPS comparison, X axis

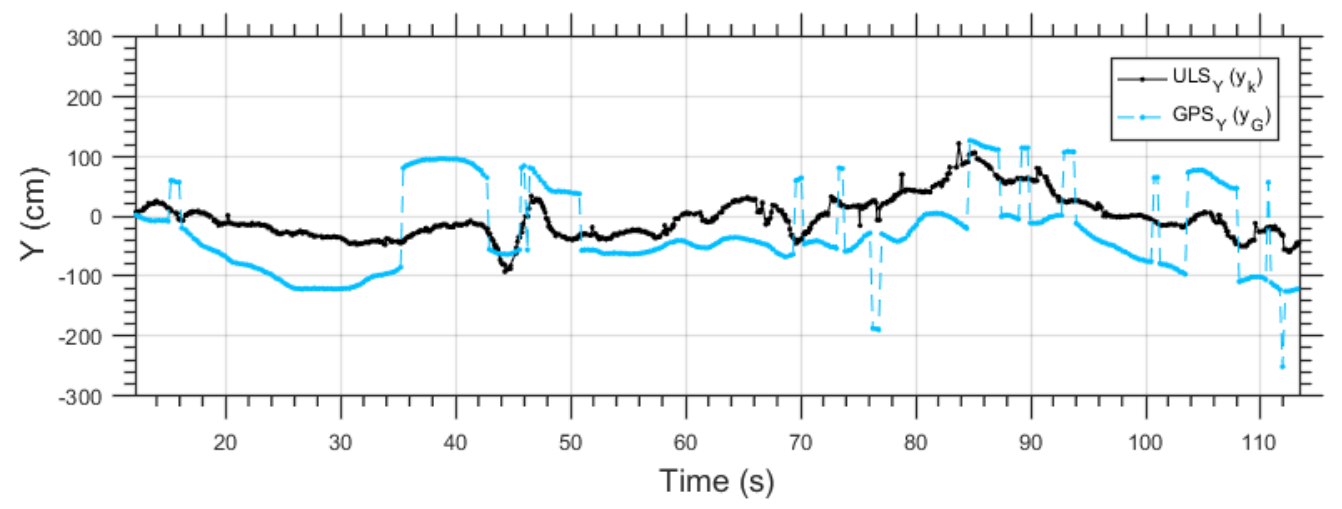

Figure 5.19: ULS - GPS comparison, Y axis

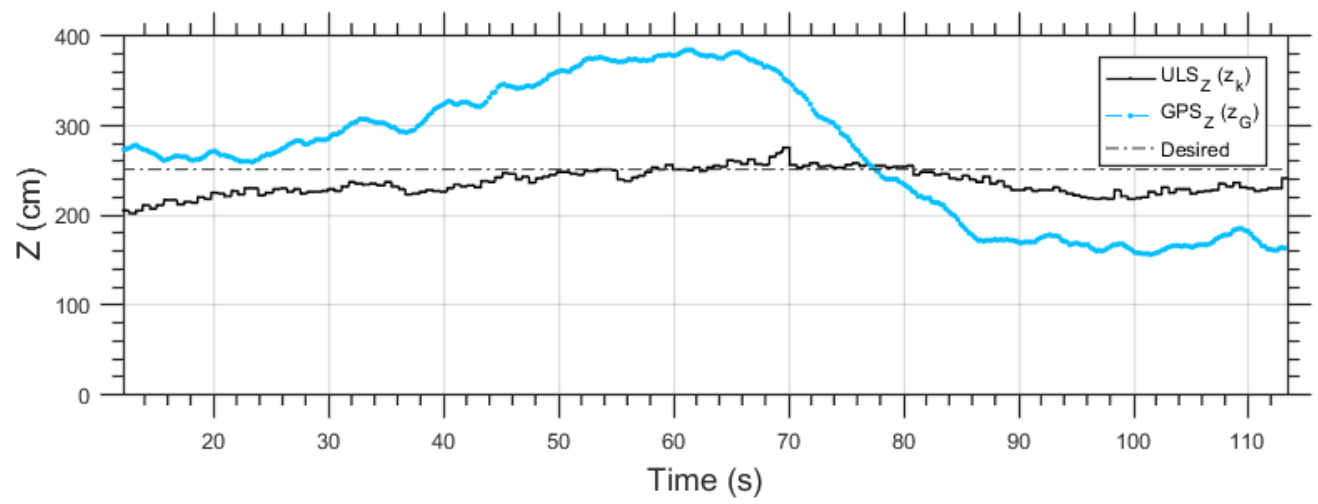

Figure 5.20: ULS - GPS comparison, Z axis 


\subsection{Portable Beacon}

The previous location for GPS denied hover and landing is too small for a portable beacon test. The portable beacon flight tests are performed in a GPS denied environment located in a gymnasium. This environment has enough space for TARA 3 to perform an autonomous hover and landing using a moving beacon. In this environment there is no external wind but there are wind currents generated by the propellers. TARA 3 hovering over top of the beacon is shown in Fig. 5.21, where the beacon is below TARA $3(0,0,0)$. The coordinate axes have been rotated from centered on TARA 3 to centered on the beacon for visualization purposes since it is easier to understand. There are pylons set up on the right side with $3.0 \mathrm{~m}$ increments for a total distance of $15 \mathrm{~m}$. For each test TARA 3 is manually positioned above the beacon to an approximate position of $(0,0,200)$ and set to autonomous mode as per section 3.2.3. After the beacon is pulled in a straight line in the positive $\mathrm{X}$ direction at a constant rate of $0.3 \mathrm{~m} / \mathrm{s}(1 \mathrm{~km} / \mathrm{hr})$. The beacon is manually pulled using a 6.0 $\mathrm{m}$ rope for safety. The beacon is angled slightly backwards such that it points in the direction of TARA 3 while following behind the beacon.

\subsubsection{Hover}

The performance of an autonomous hover using the ULS is shown in Fig. 5.22. TARA 3 is manually positioned above the beacon and set to autonomous mode at the start of the figure $(\approx 18 \mathrm{~s})$ where it tries to control to a position $(0,0,200)$ above the beacon in centimeters. At $21 \mathrm{~s}$ the beacon begins to move forward (positive $\mathrm{x}$ ) at an average speed of $0.3 \mathrm{~m} / \mathrm{s}$. This causes TARA 3 to initially drift behind in the negative $\mathrm{X}$

direction, however in the $\mathrm{X}$ direction it is able to catch up and stay within $1 \mathrm{~m}$ of the beacon. At $72 \mathrm{~s}$ the beacon has moved $15 \mathrm{~m}$ and is stopped. TARA 3 continues to hover above the beacon for the remainder of the flight. TARA 3 is successfully 


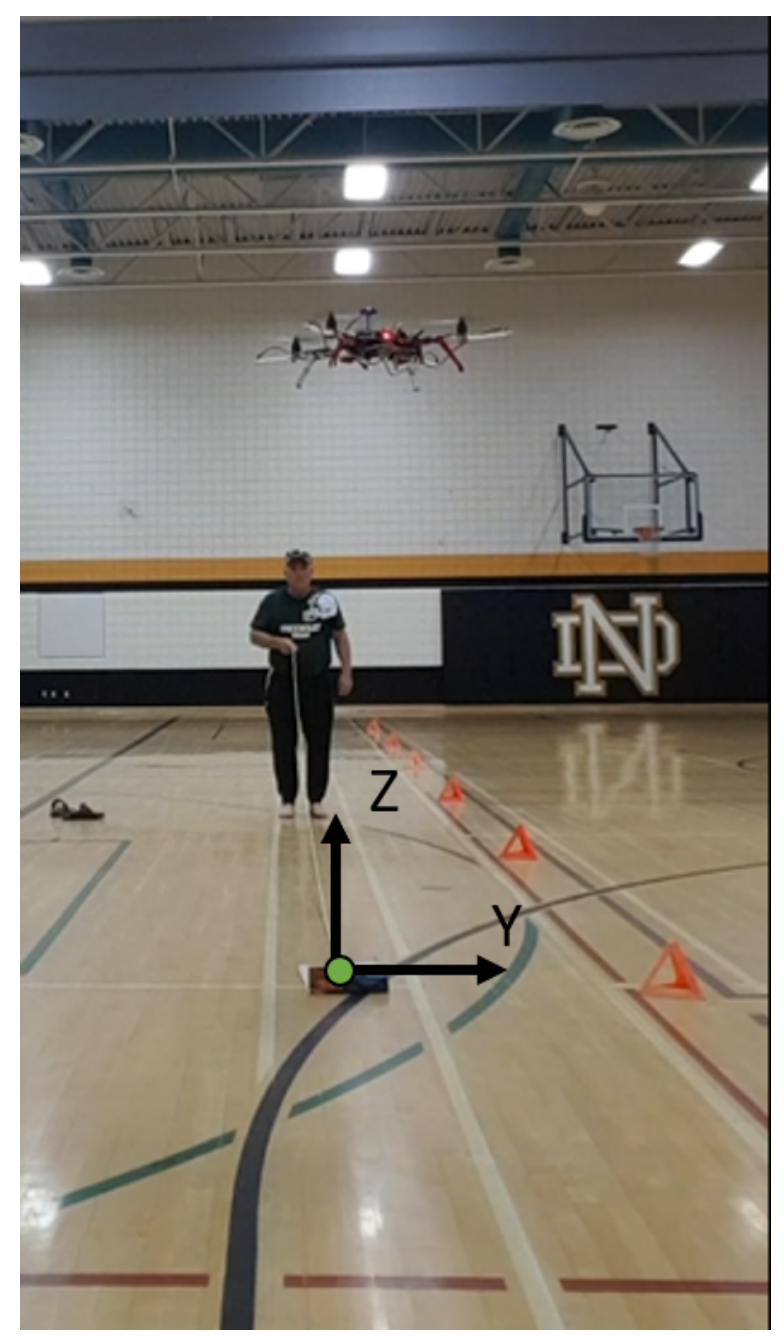

Figure 5.21: Portable test setup (X into the page)

able to track the portable beacon with a RMS error along the X axis of $45.4 \mathrm{~cm}$ and $27.4 \mathrm{~cm}$ along the $\mathrm{Y}$ axis. It makes sense the $\mathrm{X}$ axis has a larger RMS error since this is the direction of travel of the beacon. The moving beacon RMS error is higher than the indoor extended hover with the stationary beacon (RMSE along the X of $23.8 \mathrm{~cm}$ and RMSE along the $\mathrm{Y}$ of $40.6 \mathrm{~cm}$ ). This shows the moving beacon had an impact on the results but is still able to successfully track the beacon. The percentage of good signals has increased to $90 \%$ from $83 \%$ in the indoor extended hover with the stationary beacon. This could be related to the orientation of the beacon which 
points towards TARA 3 for the majority of the flight. This decreases the beam angle which increases the accuracy. For the altitude TARA 3 is initially started from an altitude of approximately $115 \mathrm{~cm}$ and tries to control up to the desired height of 200 $\mathrm{cm}$. When the beacon starts moving at $21 \mathrm{~s}$ the altitude begins to drop as TARA 3 must pitch forward to catch up to the beacon but begins to ascend at approximately $34 \mathrm{~s}$ towards the desired altitude. The altitude has a RMS error of $48.6 \mathrm{~cm}$ mostly due to initializing the height below the desired height.

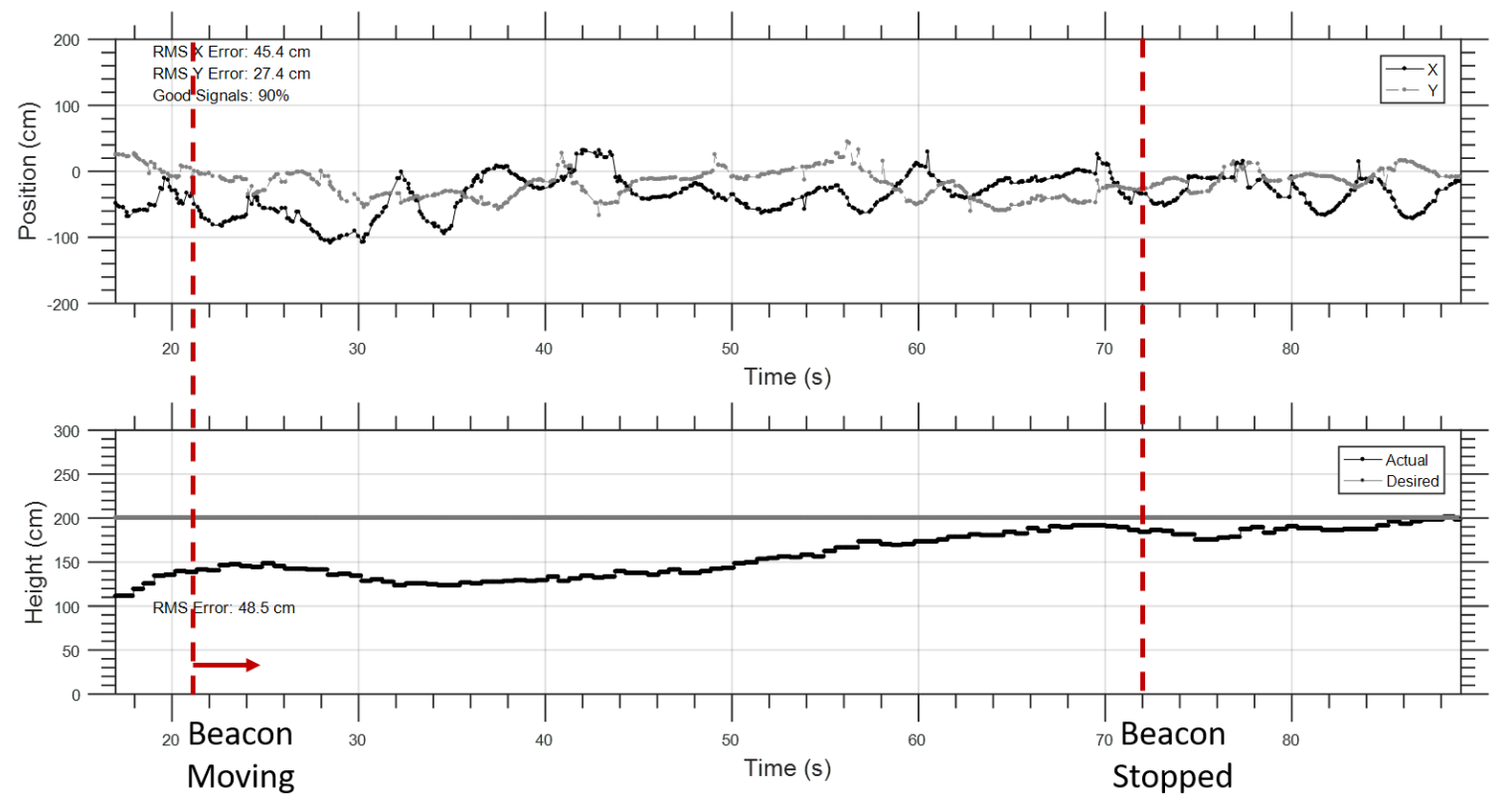

Figure 5.22: Portable beacon autonomous hover

\subsubsection{Landing}

An autonomous landing is attempted using the portable beacon as shown in Fig. 5.23. TARA 3 is manually positioned above the beacon and at $17 \mathrm{~s}$ is switched to autonomous mode (start of the figure). As TARA 3 hovers over the beacon and drifts towards the negative $\mathrm{X}$ and $\mathrm{Y}$ direction due to IMU bias. Calibrations are attempted to remove this IMU bias but are unsuccessful. This IMU bias is attributed 
to magnetic distortions from testing indoors. It has a lower impact during the hover test as it is the first test conducted indoors. The beacon begins moving in the positive $\mathrm{X}$ direction at $29 \mathrm{~s}$ which causes TARA 3 to drift further behind. However, TARA 3 is able to control itself along the $\mathrm{X}$ and $\mathrm{Y}$ axis to within the acceptable radius shown as dotted lines in the XY position graph at approximately $42 \mathrm{~s}$. As TARA 3 is within the acceptable radius, it begins to descend at a rate of $35 \mathrm{~cm} / \mathrm{s}$. For the remainder of the flight TARA 3 remains within the acceptable radius as shown with the large shaded region. A dashed dot line indicates the final descent height $(80 \mathrm{~cm})$ which TARA 3 reaches before 42 seconds and thus continues to descend regardless of position as shown with the smaller shaded region. The dashed double dot line shows the final height which the sonar can detect which is reached just before 49 seconds. The final distance from the beacon to the center of the quadrotor is measured to be $21 \mathrm{~cm}$ or $26.3 \%$ of the wingspan. The RMS error is $47.2 \mathrm{~cm}$ for the $\mathrm{X}$ axis and $50.8 \mathrm{~cm}$ for the $\mathrm{Y}$ axis. It is expected that the $\mathrm{X}$ axis has a larger RMS error since TARA 3 is following behind the beacon in the $\mathrm{X}$ direction but this anomaly is related to the IMU bias as previously discussed. The RMS error is greater than the indoor test with the stationary beacon (Fig 5.16) with a RMS error along the X axis of 10.3 $\mathrm{cm}$ and $47.9 \mathrm{~cm}$ along the $\mathrm{Y}$ axis. This makes sense with the IMU bias issues and using a moving beacon. The percentage of successful measurements for the moving beacon increased to $93 \%$ versus $86 \%$ with the stationary beacon most likely due to the orientation of the beacon as previously discussed.

These tests have shown that TARA 3 can successfully hover and land using a portable beacon in a GPS denied environment. Faster beacon speeds are not explored because staying within the cone of the beacon becomes more of a controller based issue. Whereas this thesis focuses on the localization aspect while using a sufficient controller to achieve the desired missions. The controller issue is highlighted in Appendix B where ArduCopter's built in controller has significantly better performance 


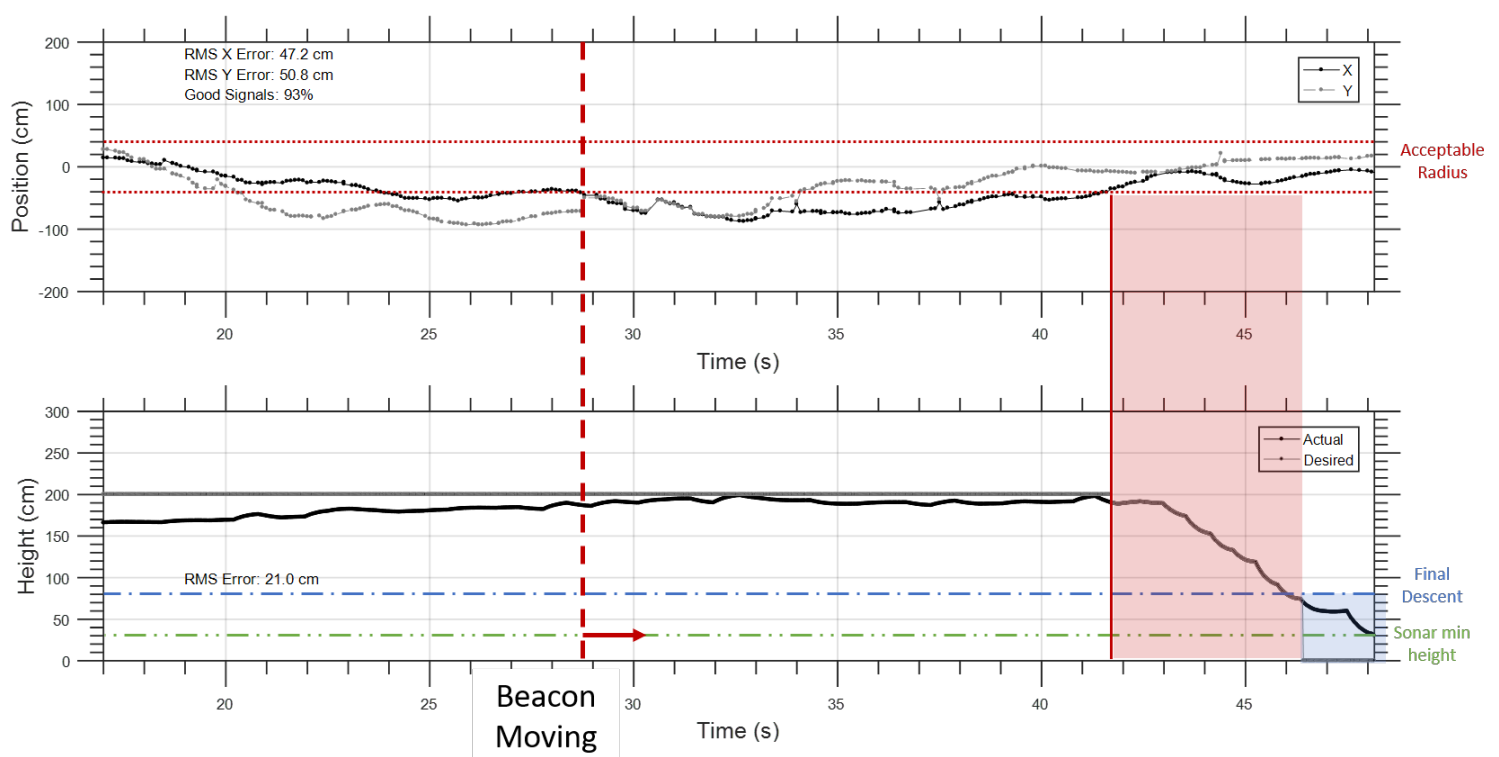

Figure 5.23: Portable beacon autonomous landing

using the raw GPS signal despite the raw GPS signal having worse performance than the ULS. 


\section{Chapter 6}

\section{Conclusion}

This thesis presents a method for localization in a GPS-denied environment. Based on the requirements, ultrasound is selected for the localization of a quadrotor. A gap in the research led to the concept of using a portable beacon by mounting the receivers on the quadrotor. The selected system uses ultrasound which limits the detection range but is able to provide accurate results based on similar systems. Time

of flight is selected as the ranging technique because it provides accurate distance measurements with the speed of sound. Thereshold detection is used to provide fast measurements. The analytical and the linear least square lateration methods are explored and compared.

The system accuracy is assessed by mounting TARA 3 in the ceiling and measuring the position of the beacon at set locations. The five-receiver linear least squares is more accurate than the trilateration method by $31 \%$. However, it receives $5 \%$ fewer successful measurements because it requires more distance measurements. GPSdenied flight tests are conducted and again the five-receiver LLS algorithm performs the best. It outperforms the hover performance of the trilateration method with a $23 \%$ better average root mean square error. However, the five-receiver LLS algorithm receives $2 \%$ fewer successful measurements due to the extra distance measurements. 
The five-receiver LLS has the greatest accuracy but does not have a sufficient update rate. A combined lateration algorithm is developed in order to perform quadrotor localization. The combined lateration algorithm outperforms the other methods with an average standard deviation of $8.0 \mathrm{~cm}$ and an average number of successful measurements of $93 \%$. The combined lateration algorithm has superior hover performance relative to the other algorithms with a root mean square error of $51.3 \mathrm{~cm}$ and an average percentage of successful measurements of $81 \%$. The combined lateration algorithm completes autonomous landings to a beacon within $22.5 \%$ of the wingspan $(18 \mathrm{~cm})$.

The combined lateration algorithm has superior performance than the GPS in terms of stationary accuracy and update rate. During an outdoor hover controlled by the ULS, the raw GPS position data drifts and is noisier than the data from the ULS.

TARA 3 is successfully able to hover and land using a portable beacon in a GPS denied environment. TARA 3 is able to track the beacon along a linear path over a distance of $15 \mathrm{~m}$ with similar accuracy and update rate as the stationary beacon hover tests. TARA 3 is also able to successfully land on the moving beacon travelling on a linear path with similar accuracy and update rate as the stationary beacon tests.

\subsection{Summary of Contributions}

- Developed a portable ultrasonic localization system capable of localizing a quadrotor in a GPS denied environment. Performed autonomous hover and landing using a stationary and moving beacon in a GPS-denied environment.

- Developed a combined lateration algorithm which performs localization using five, four or three distance measurements. This combines the improved accuracy of linear least squares with the improved update rate of analytical trilateration 
to achieve a better performance than using an individual algorithm.

- Achieved better accuracy and a higher update rate than a standard GPS.

- Integrated the system with a commercial product, the Pixhawk, which demonstrates this product can be placed on most drones for localization.

\subsection{Future Research}

- The key limitation to the ultrasonic localization system is the detection distance. Ultra-wideband presents a method which has a high accuracy and a detection range of over $200 \mathrm{~m}$ in an open environment. The ranging technique, lateration technique and controls can be identical as the ultrasonic system. If the same receiver locations are used, the dilution of precision must be considered as the quadrotor distance increases.

- Large scale ultrasonic localization can be obtained by using multiple beacons. Using correlation techniques, each beacon can be assigned a unique identifier which identifies which beacon is communicating with the quadrotor. As the quadrotor travels it will know its position based on the calculated position through lateration and the position of the beacon which it uses for lateration.

- Currently a Kalman filter is used to reduce noise in the position data but the position accuracy can be improved by integrating the ultrasonic position and IMU into an extended Kalman filter. This can allow for a higher position update rate as the IMU will provide position updates between the ULS position updates. In addition an EKF can be used to estimate the velocity of the beacon which can be used in a controller. Attempts were made to inject the ultrasonic signal as a GPS signal into ArduCopter which would take advantage of ArduCopter's EKF. However, noisy measurements caused the extended Kalman filter to diverge. 
- The controller is designed to work for a stationary beacon or a slow moving beacon. For higher beacon speeds the controller should be optimized for a moving beacon.

- Indoor environments contain magnetic distortions which cause the IMU magnetometer to have issues. The magnetometer is responsible for providing yaw information, but the ULS can also provide yaw information if a second beacon is placed at a fixed distance from the first beacon. This would remove the requirement for a magnetometer and allows for indoor localization unaffected by magnetic distortions.

- When testing outdoors wind has a large influence on the control of TARA 3, to the point where outdoor tests needed to be conducted in a near wind free environment. To improve this, the hybrid controller presented by Ali Raza [72], designed to optimize control in an urban wind environment should be implemented. 


\section{List of References}

[1] W. J. H. T. Center. "Global Positioning System (GPS) Standard Positioning Service (SPS) Performance Analysis Report." (March), 1-61 (2014).

[2] Murata Manufacturing Co. Ltd. "Ultrasonic Sensor Application Manual." pages $1-15$ (2008).

[3] J. Gundlach. "Designing Unmanned Aircraft Systems." (2012).

[4] J. Laliberte. "MECH 5805 Uninhabited Aircraft Systems." (2017).

[5] J. Feist. "Military drones - the new air force - DroneRush." (2019).

[6] R. Struthers. "Drone Delivery Canada Leaps Ahead Of Amazon Prime Air And Google Wing - Drone Delivery Canada Corp. (OTCMKTS:TAKOF) — Seeking Alpha." (2019).

[7] T. Reuters. "Google's Wing Aviation gets OK for drone deliveries in U.S. CBC News." (2019).

[8] DroneDeliveryCanada. "Drone Delivery Canada Supports Transport Canada Drone Delivery Canada: Drone Delivery Canada.” (2019).

[9] M. Margaritoff. "Transport Canada Selects Fortem Technologies to Join BVLOS Drone Flight Trials - The Drive." (2018).

[10] LiveViewGPS. "GPS and its Three Main Competitors: Galileo, Beidou, GLONASS - Live View GPS Tracking Blog." (2014).

[11] O. T. Martí. "Study of differential GPS system for UAVs." (2016).

[12] U. Shah, R. Khawad, and K. M. Krishna. "DeepFly: Towards Complete Autonomous Navigation of MAVs with Monocular Camera." Proceedings of the Tenth Indian Conference on Computer Vision, Graphics and Image Processing - ICVGIP '16 pages 1-8 (2016). 
[13] P. K. Enge. "The Global Positioning System: Signals, measurements, and performance." International Journal of Wireless Information Networks 1(2), 83-105. ISSN 10689605 (1994).

[14] N. Aleksander, K. Kamil, D. Krzysztof, and K. Roman. "Differential navigation for UAV platforms with mobile reference station." Selected Topics in Applied Computer Science pages 465-471. ISSN 17924863 (2010).

[15] M. Gowda, J. Manweiler, A. Dhekne, R. R. Choudhury, and J. D. Weisz. "Tracking drone orientation with multiple GPS receivers." Proceedings of the 22nd Annual International Conference on Mobile Computing and Networking - MobiCom '16 pages 280-293 (2016).

[16] T. H. Witte and A. M. . Wilson. "Accuracy of WAAS-enabled GPS for the determination of position and speed over ground." 38, 1717-1722 (2005).

[17] Kairos Autonomi. "GPS Correction Comparisons: RTK versus DGPS.” (2014).

[18] S. Tansuriyong, M. Kyan, K. Numata, S. Taira, and T. Anezaki. "Verification Experiment for Drone Charging." pages 229-232 (2017).

[19] R. Li, Z. Du, Y. Zhao, and S. Liu. "Design and Implementation of Mobile Robot Ultrasonic Localization System." 28th Chinese Control and Decision Conference (CCDC) pages 5347-5352 (2016).

[20] F. Kendoul. "Survey of Advances in Guidance , Navigation , and Control of Unmanned Rotorcraft Systems." 29(2) (2012).

[21] C. Martínez, I. F. Mondragón, M. A. Olivares-Méndez, and P. Campoy. "Onboard and ground visual pose estimation techniques for UAV control." Journal of Intelligent and Robotic Systems: Theory and Applications 61(1-4), 301-320. ISSN 09210296 (2011).

[22] S. Lange, N. Sunderhauf, and P. Protzel. "A vision based onboard approach for landing and position control of an autonomous multirotor UAV in GPSdenied environments." Proceedings of the International Conference on Advanced Robotics pages 1-6 (2009).

[23] M. Bošnak, D. Matko, and S. Blažič. "Quadrocopter hovering using positionestimation information from inertial sensors and a high-delay video system." Journal of Intelligent and Robotic Systems: Theory and Applications 67(1), 4360. ISSN 09210296 (2012). 
[24] F. Cocchioni, E. Frontoni, G. Ippoliti, S. Longhi, A. Mancini, and P. Zingaretti. "Visual Based Landing for an Unmanned Quadrotor." Journal of Intelligent and Robotic Systems: Theory and Applications 84(1-4), 511-528. ISSN 15730409 (2016).

[25] Y. H. Lee and J. S. Chahl. "Design and implementation of a vision-based hovering and feature tracking algorithm for a quadrotor." IOP Conference Series: Materials Science and Engineering 152(1). ISSN 1757899X (2016).

[26] E. Johnson, A. Calise, Y. Watanabe, J. Ha, and J. Neidhoefer. "Real-timevisionbasedrelativeaircraftnavigation." AIAA Journal of Aerospace Computing, Information, and Communication 4(4), 707-738 (2007).

[27] L. Wang and X. Bai. "Quadrotor Autonomous Approaching and Landing on a Vessel Deck." Journal of Intelligent and Robotic Systems: Theory and Applications (December), 1-19. ISSN 15730409 (2017).

[28] M. Robotics. "Marvelmind Indoor Navigation System Operating Manual." .

[29] F. Zafari, A. Gkelias, and K. Leung. "A Survey of Indoor Localization Systems and Technologies." pages 1-30 (2017).

[30] Government of Canada. "Devices Using Ultra-Wideband ( UWB ) Technology." $\mathbf{1}(1)$ (2018).

[31] A. Alarifi, A. Al-Salman, M. Alsaleh, A. Alnafessah, S. Al-Hadhrami, M. AlAmmar, and H. Al-Khalifa. "Ultra Wideband Indoor Positioning Technologies: Analysis and Recent Advances." Sensors 16(5), 707. ISSN 1424-8220 (2016).

[32] M. M. Saad, C. Bleakley, and S. Dobson. "Robust High-Accuracy Ultrasonic Range Measurement System." IEEE Transactions on Instrumentation and Measurement 60(10). ISSN 10534822 (2011).

[33] J. M. Martín, A. R. Jiménez, F. Seco, L. Calderón, J. L. Pons, and R. Ceres. "Estimating the 3D-position from time delay data of US-waves: Experimental analysis and a new processing algorithm." Sensors and Actuators, A: Physical 101(3), 311-321. ISSN 09244247 (2002).

[34] R. Want, A. Hopper, V. Falcão, and J. Gibbons. "The active badge location system." ACM Transactions on Information Systems 10(1), 91-102. ISSN 10468188 (1992). 
[35] A. Harter, A. Hopper, P. Steggles, A. Ward, and P. Webster. "The anatomy of a context-aware application." Wireless Networks 8(2-3), 187-197. ISSN 10220038 (2002).

[36] N. B. Priyantha, A. Chakraborty, and H. Balakrishnan. "The Cricket locationsupport system." Proceedings of the 6th annual international conference on Mobile computing and networking - MobiCom '00 2000(August), 32-43. ISSN 09574174 (2000).

[37] J. Eckert, K. Koeker, P. Caliebe, F. Dressler, and R. German. "Self-localization capable mobile sensor nodes." 2009 IEEE International Conference on Technologies for Practical Robot Applications, TePRA 2009 pages 224-229 (2009).

[38] J. Eckert, F. Dressler, and R. German. "Real-time indoor localization support for four-rotor flying robots using sensor nodes." 2009 IEEE International Workshop on Robotic and Sensors Environments, ROSE 2009 - Proceedings pages 23-28 (2009).

[39] J. Eckert, R. German, and F. Dressler. "An indoor localization framework for four-rotor flying robots using low-power sensor nodes." IEEE Transactions on Instrumentation and Measurement 60(2), 336-344. ISSN 00189456 (2011).

[40] J. A. Paredes, F. J. Álvarez, T. Aguilera, and J. M. Villadangos. "3D indoor positioning of UAVs with spread spectrum ultrasound and time-of-flight cameras." Sensors (Switzerland) 18(1), 1-15. ISSN 14248220 (2018).

[41] B. Katona and K. Morgansen. "Navigation of Indoor Spaces Using Multiple Quadrotors." (January), 1-25 (2019).

[42] T.-s. Nguyen, T.-n. Nguyen, Q.-s. Tran, and T.-h. Huynh. "Improvement of Ultrasound-Based Localization System Using Sine Wave Detector and CAN Network." Journal of Sensor and Actuator Networks 6(3), 12. ISSN 2224-2708 (2017).

[43] J. Qi and G.-P. Liu. "A Robust High-Accuracy Ultrasound Indoor Positioning System Based on a Wireless Sensor Network." Sensors 17(11), 2554. ISSN 1424-8220 (2017).

[44] C. Medina, J. C. Segura, and . De la Torre. "Ultrasound indoor positioning system based on a low-power wireless sensor network providing sub-centimeter accuracy." Sensors (Switzerland) 13(3), 3501-3526. ISSN 14248220 (2013). 
[45] A. De Angelis, A. Moschitta, P. Carbone, M. Calderini, S. Neri, R. Borgna, and M. Peppucci. "Design and Characterization of a Portable Ultrasonic Indoor 3-D Positioning System." IEEE Transactions on Instrumentation and Measurement 64(10), 2616-2625. ISSN 00189456 (2015).

[46] O. Bischoff, N. Heidmann, J. Rust, and S. Paul. "Design and implementation of an ultrasonic localization system for wireless sensor networks using angle-ofarrival and distance measurement." Procedia Engineering 47, 953-956. ISSN 18777058 (2012).

[47] M. O. Khyam, L. Xinde, S. S. Ge, and M. R. Pickering. "Multiple Access Chirp-Based Ultrasonic Positioning." IEEE Transactions on Instrumentation and Measurement 66(12), 3126-3137. ISSN 00189456 (2017).

[48] A. Benini, A. Mancini, and S. Longhi. "An IMU/UWB/vision-based extended kalman filter for mini-UAV localization in indoor environment using 802.15.4a wireless sensor network." Journal of Intelligent and Robotic Systems: Theory and Applications 70(1-4), 461-476. ISSN 09210296 (2013).

[49] A. K. Raja and Z. Pang. "High accuracy indoor localization for robot-based fine-grain inspection of smart buildings." Proceedings of the IEEE International Conference on Industrial Technology 2016-May, 2010-2015 (2016).

[50] M. Segura, V. Mut, and C. Sisterna. "Ultra wideband indoor navigation system." IET Radar Sonar \& Navigation 6(5), 402. ISSN 17518784 (2012).

[51] N. Kirchner and T. Furukawa. "Infrared localisation for indoor uavs." ICST05: Proceedings of the 2005 International Conference on Sensing Technology pages 60-65 (2005).

[52] J. Lategahn, M. Müller, and C. Röhrig. "TDoA and RSS based extended kalman filter for indoor person localization." IEEE Vehicular Technology Conference pages 0-4. ISSN 15502252 (2013).

[53] V. C. Paterna, A. C. Augé, J. P. Aspas, and M. A. P. Bullones. "A bluetooth low energy indoor positioning system with channel diversity, weighted trilateration and kalman filtering." Sensors (Switzerland) 17(12). ISSN 14248220 (2017).

[54] D. Zhang, L. T. Yang, M. Chen, S. Zhao, and S. Member. "06891174." pages 1-10 (2014).

[55] Optitrack. "OptiTrack Motion Capture." (2018). 
[56] Pozyx. "Pozyx Accurate Positioning."

[57] J. Eckert, R. German, and F. Dressler. "On autonomous indoor flights: Highquality real-time localization using low-cost sensors." IEEE International Conference on Communications pages 7093-7098. ISSN 15503607 (2012).

[58] I. Inc. "MPU-6000 and MPU-6050 Product Specification." InvenSense Inc. $\mathbf{1}(408), 1-57$ (2013).

[59] ST-Microelectronics. "L3GD20H: 3-axis digital output gyroscope." (April 2015) (2013).

[60] ST-Microelectronics. "Lsm303D eCompass module." (November), 1-52 (2013).

[61] T. E. S. Solutions. "MS5611-01BA03." m (2017).

[62] Ardupilot. "ArduPilot Codebase." (2016).

[63] Ublox. "Neo-M8." (2016).

[64] Microstar. "Ziegler-Nichols Tuning Rules for PID."

[65] MaxBotix Inc. "Ultrasonic Sensor Operation on a UAV." (2017).

[66] C. Fleischer. "How Piezoelectricity Works."

[67] Murata. "MA40S4S/MA40S4R Application Note." (Dm), 1-13.

[68] Belden. "Belden 8451 Shielded Wire." .

[69] MaxBotix Inc. "Datasheet XL-MaxSonar - EZ Series." pages 1-16 (2015).

[70] R. Zhang, F. Höflinger, and L. Reindl. "TDOA-Based localization using interacting multiple model estimator and ultrasonic transmitter/receiver." IEEE Transactions on Instrumentation and Measurement 62(8), 2205-2214. ISSN 00189456 (2013).

[71] W. Selby. "ArduCopter." (2014).

[72] S. A. Raza. "Autonomous UAV Control for Low-Altitude Flight in an Urban Gust Environment by." (August) (2015).

[73] J. Etele. "Aircraft Performance Lecture Notes." Technical report (2016). 


\section{Appendix A}

\section{Additional Accuracy Tests}

Additional accuracy tests are performed to assess the impact of various parameters which could effect the noise of the measurements.

\section{A.1 Beacon Setup}

The beacon setup is tested using a single transducer versus using five transducers mounted on a hemisphere. A single transducer is limited to a cone of $45^{\circ}$ due to directivity attenuation. Therefore, multiple transducers provides full hemispherical coverage which potentially decreases the directivity attenuation of the beacon (there is still attenuation on the receiving side due to the receiving angle). The position accuracy for a single transducer beacon is shown in Fig. A.1 (same figure as Fig. 5.4) and the for the 5 transducer beacon in Fig. A.2. The full beacon has very poor mean measurements at point $\mathrm{F}$ and I most likely due to wave interference. The single transducer beacon has a $53 \%$ better RMSE, a $52 \%$ better average standard deviation than the 5 transducer beacon. It also has $8 \%$ more successful measurements. It outperforms the 5 transducer beacon at nearly every location. By using multiple transducers in the beacon there is interference between the ultrasonic signals sent. Any constructive or destructive interference leads to distance measurements that fail 
the filter tests described in section 4.3 .5 and so a single transducer beacon is used for all testing.

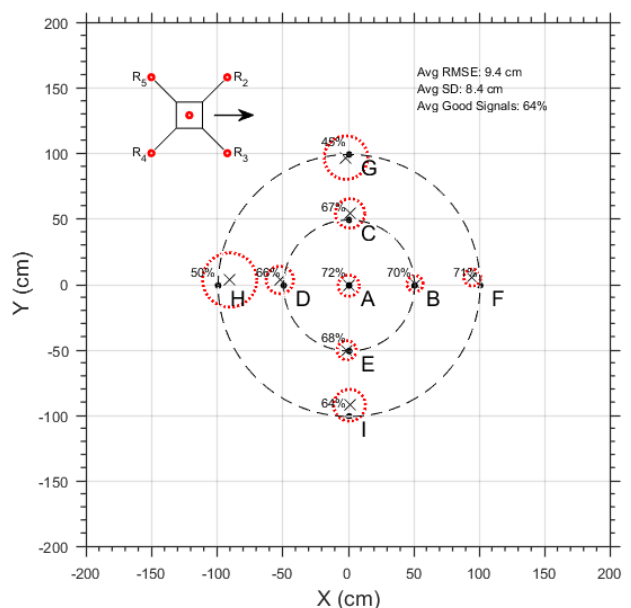

Figure A.1: Position accuracy of a single transducer

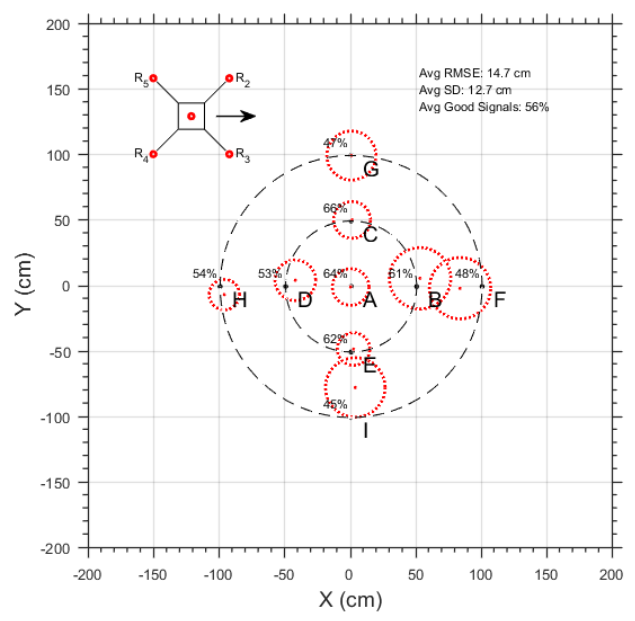

Figure A.2: Position accuracy of the full beacon

\section{A.2 Propellers Running}

An additional test is conducted with the motors off to determine the effect the propellers have on the measurements. To simulate in flight dynamics such as vibrations and turbulence the propellers operate at half the required hover throttle. The position accuracy results are shown with the propellers in Fig. A.3 (same figure as Fig. 5.4) and without in Fig. A.4. The motors off have a $3.7 \%$ better average standard deviation than the propellers on but a $5 \%$ worse average RMSE. The motors off receives $10 \%$ more successful measurements than the propellers on. At nearly all the locations the motors off case outperforms the propellers on. This shows that the EMI, vibrations and turbulence have an impact on the results. The worse RMSE could be due to an improper setup of the nominal positions as all other metrics have 
outperformed with the propellers off.

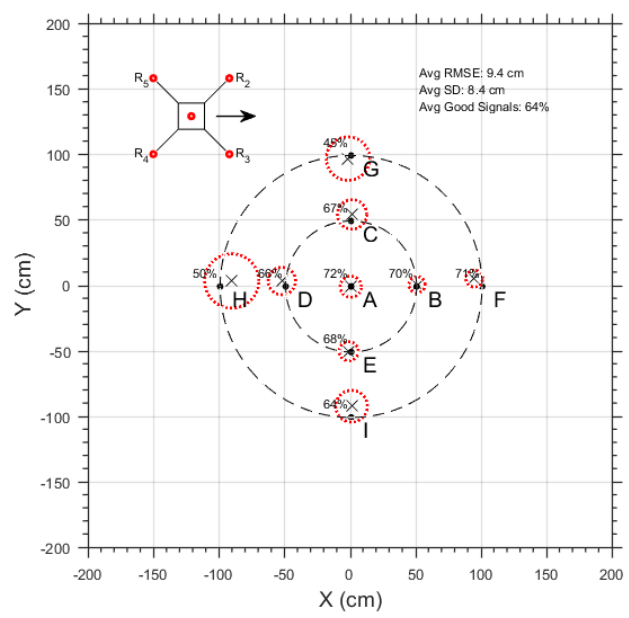

Figure A.3: Position accuracy with propellers at half hover throttle

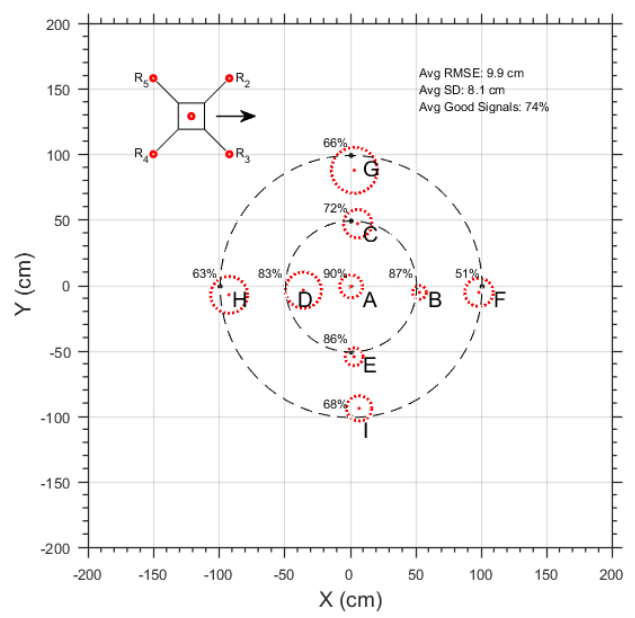

Figure A.4: Position accuracy with no motors

\section{A.3 Receiver Mounting Location on Quadrotor}

The receiver location is changed to determine the impact of mounting the receivers off or on the motor arms. Additional arms are mounted orthogonal to the motor arms in a '+' configuration as shown in Fig. A.5. The receivers remain at the same distance away from each other the dilution of precision remains constant. This reduces the EMI since the wires are not running directly next to the motors. In addition, this changes the propagation characteristics through the propeller wake. The position accuracy for the motors mounted on the arms is shown in Fig. A.6 (same figure as Fig. 5.4) and the orthogonal layout is shown in Fig. A.7. The regular setup has a $65 \%$ better RMSE, a $67 \%$ better average standard deviation and receives $3 \%$ more successful measurements than the orthogonal layout. It outperforms the orthogonal layout at most beacon locations. The poor accuracy is attributed to vibrations and inaccurate 
receiver locations. The orthogonal arms have slight lateral movement which can lead to inaccurate measurements.

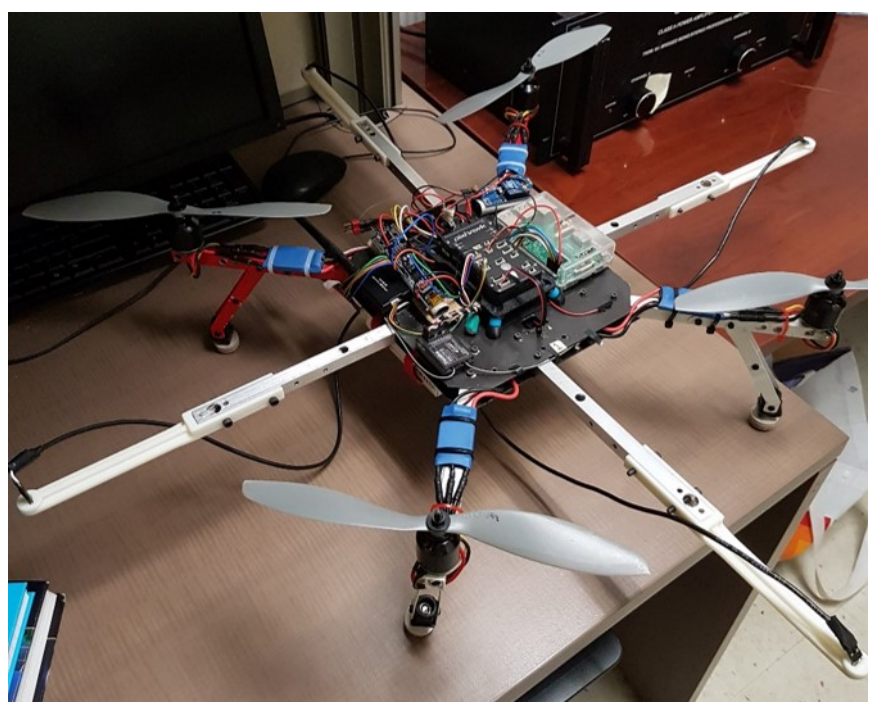

Figure A.5: Orthogonal receiver configuration 


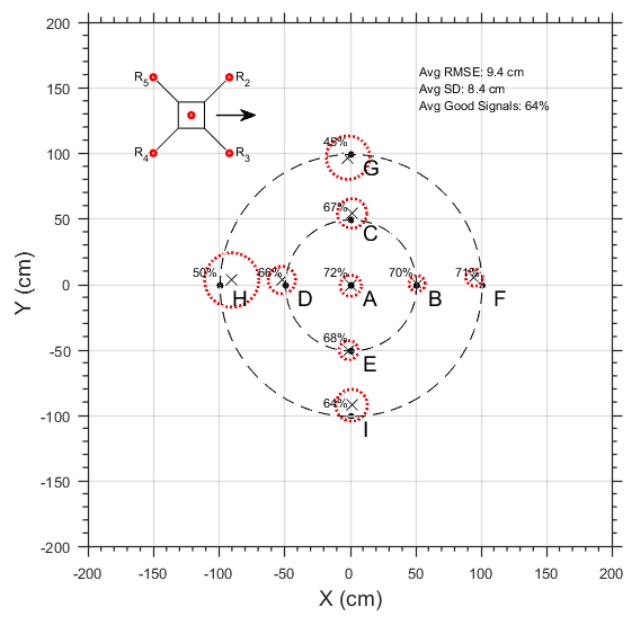

Figure A.6: Position accuracy of receivers mounted on motor arms

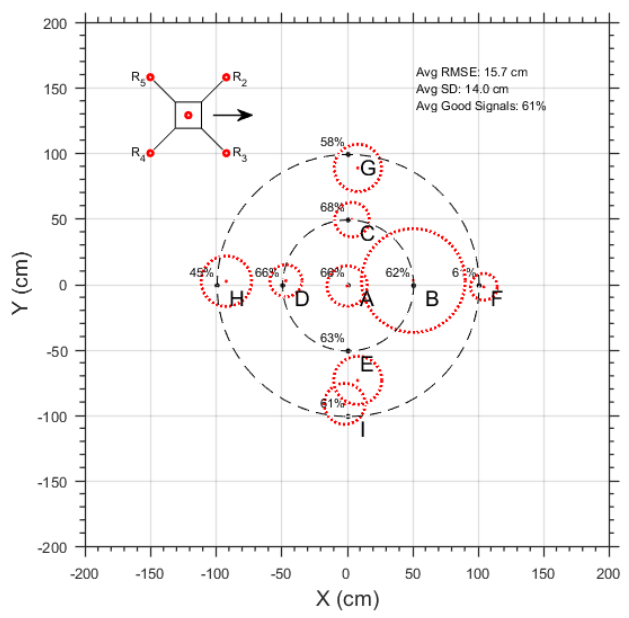

Figure A.7: Position accuracy of the orthogonal layout 


\section{Appendix B}

\section{Outdoor Flight Testing}

The ULS is compared with a GPS connected to a Pixhawk running Arducopter. The GPS system uses a superior control system built into Arducopter. It fuses the GPS signal with an IMU through an EKF to increase accuracy. The ULS uses the beacon as the target, whereas the GPS system uses a desired coordinate. They are compared using their hover and landing performance. For all ULS tests the GPS is disconnected such that the two systems are independent of each other. Having the GPS connected during the flight tests would correct for the IMU bias that develops during the ULS tests.

Tests using the raw GPS signal as an input to the control system rather than the ULS are conducted but are unsuccessful. The raw GPS signal is too noisy to be used in the same controller as the ULS.

\section{B.1 Hover}

TARA 3 is manually positioned above the beacon and set to autonomous mode where it tries to control to a position $(0,0,250)$ above the beacon in centimeters. The performance of an autonomous hover using the ULS is shown in Fig. B.1. The position $(\mathrm{X}, \mathrm{Y})$ graph is shown on top where $\mathrm{X}$ is the black line and $\mathrm{Y}$ is the grey dashed line 
representing the points $\left(x_{k}, y_{k}\right)$. At the top the wind direction is shown with arrows obtained from the weather vane in the post processed video, the axes indicate the wind direction. The system is capable of hovering over the beacon for $205 \mathrm{~s}$ before the flight leaves the $45^{\circ}$ cone due to IMU drift. The bottom graph shows the height against time with the desired height $(2.5 \mathrm{~m})$ shown as a grey dashed line. The altitude fluctuates drastically with an RMS error of $185.7 \mathrm{~cm}$ or $74.3 \%$ of the desired altitude. The sonar works better over hard surfaces, the grass causes the signal to diverge and leads to incorrect signals. This leads to the noisy performance shown in the altitude graph. This is significantly worse altitude control than the GPS-denied altitude control with a RMS error of $7.5 \mathrm{~cm}$. Based on the video performance the physical altitude does not have a RMS error of $185.7 \mathrm{~cm}$ but is caused by sonar measurement noise due to the grass surface. The position performance fluctuates within $2 \mathrm{~m}$ of the beacon and converges to the beacon location. It has a RMS error along the $\mathrm{X}$ axis of $67.0 \mathrm{~cm}$ and along the $\mathrm{Y}$ axis of $48.2 \mathrm{~cm}$ as compared to the indoor test with an RMS error along the $\mathrm{X}$ axis of $23.8 \mathrm{~cm}$ and along the $\mathrm{Y}$ axis of $40.6 \mathrm{~cm}$. This performance is worse than the indoor performance due to poor altitude control and outdoor conditions. The number of successful measurements is $82 \%$ which is comparable to the $83 \%$ in the indoor tests.

For GPS controlled hover testing TARA 3 is manually positioned over the desired coordinate and set to GPS hold where it uses its built-in control system to maintain its position. The GPS hold function is covered in Section 3.2.3. The desired position is set to the GPS position when it is switched from manual control to GPS hold. The performance using the GPS system is shown in Fig. B.2 where the desired position is $(0,0,253)$. The graph has been shifted such that the desired position lies on the 0 axis for the $\mathrm{x}$ and $\mathrm{y}$ directions. This system has a RMS error along the $\mathrm{X}$ axis of $20.4 \mathrm{~cm}$ and of $5.6 \mathrm{~cm}$ along the $\mathrm{Y}$ axis. There may have been an initial velocity towards negative $\mathrm{x}$ which led to a higher RMS value as the wind should not have 


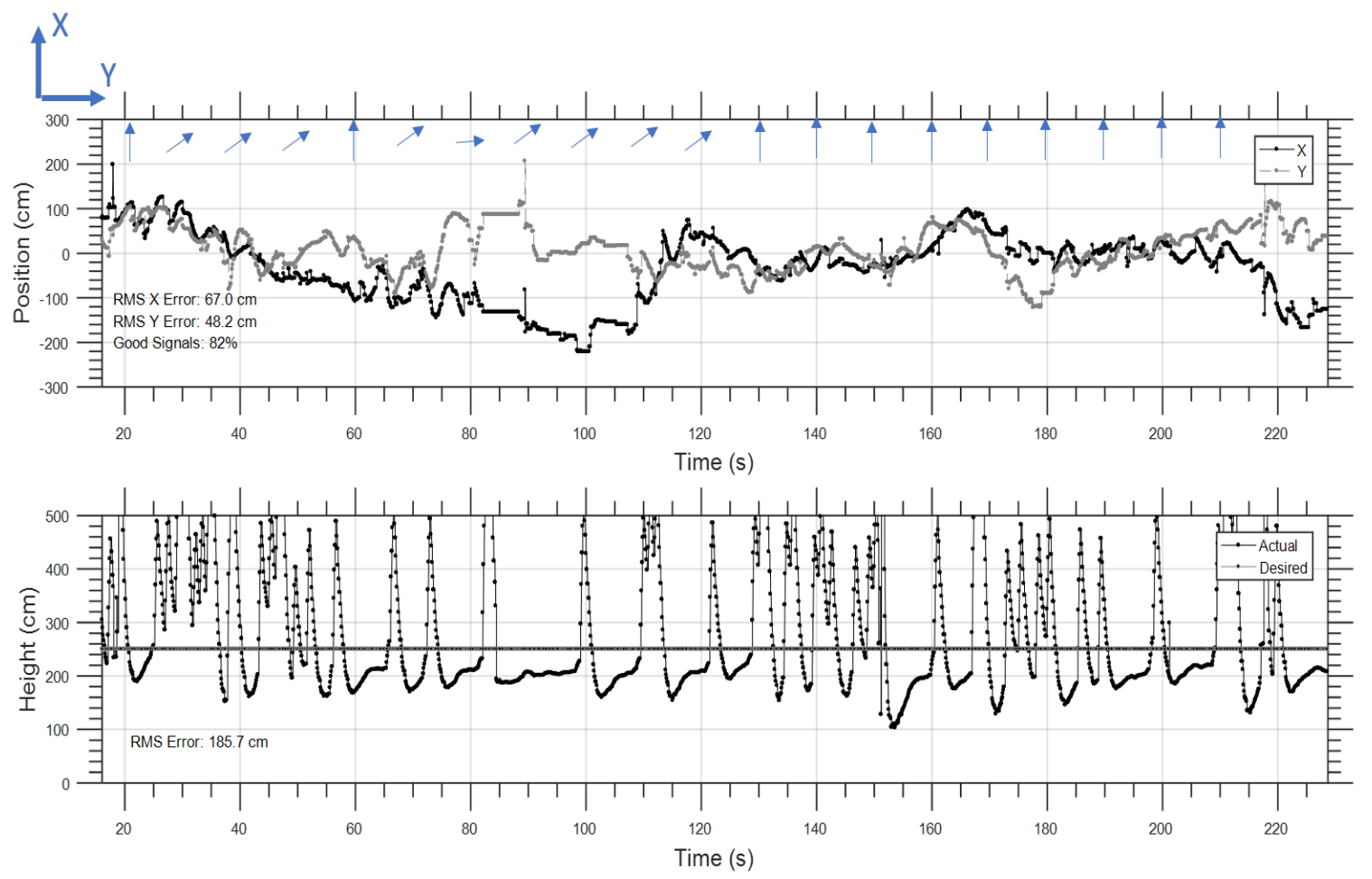

Figure B.1: Hover in an outdoor environment using the ULS

contributed in that direction. The $\mathrm{Z}$ axis has good control with an RMS error of only $9.0 \mathrm{~cm}$ as compared to the sonar outdoors $(185.7 \mathrm{~cm})$. The GPS outperforms the ULS for hover and this can be related to poor altitude measurements and the fusion of multiple sensors through ArduCopter's EKF yielding a superior performance.

\section{B.2 Landing}

For the ULS landing, TARA 3 follows the same landing logic as in Fig. 5.14. TARA 3 starts above the beacon trying to hover at a desired altitude of $2.5 \mathrm{~m}$. Similar to the hover performance, there is significant fluctuation in the height due to measurement noise. The desired land velocity is set at $35 \mathrm{~cm} / \mathrm{s}$. The figure starts after it has been switched to autonomous mode at $15 \mathrm{~s}$. TARA 3 tries to hover over top of the beacon 

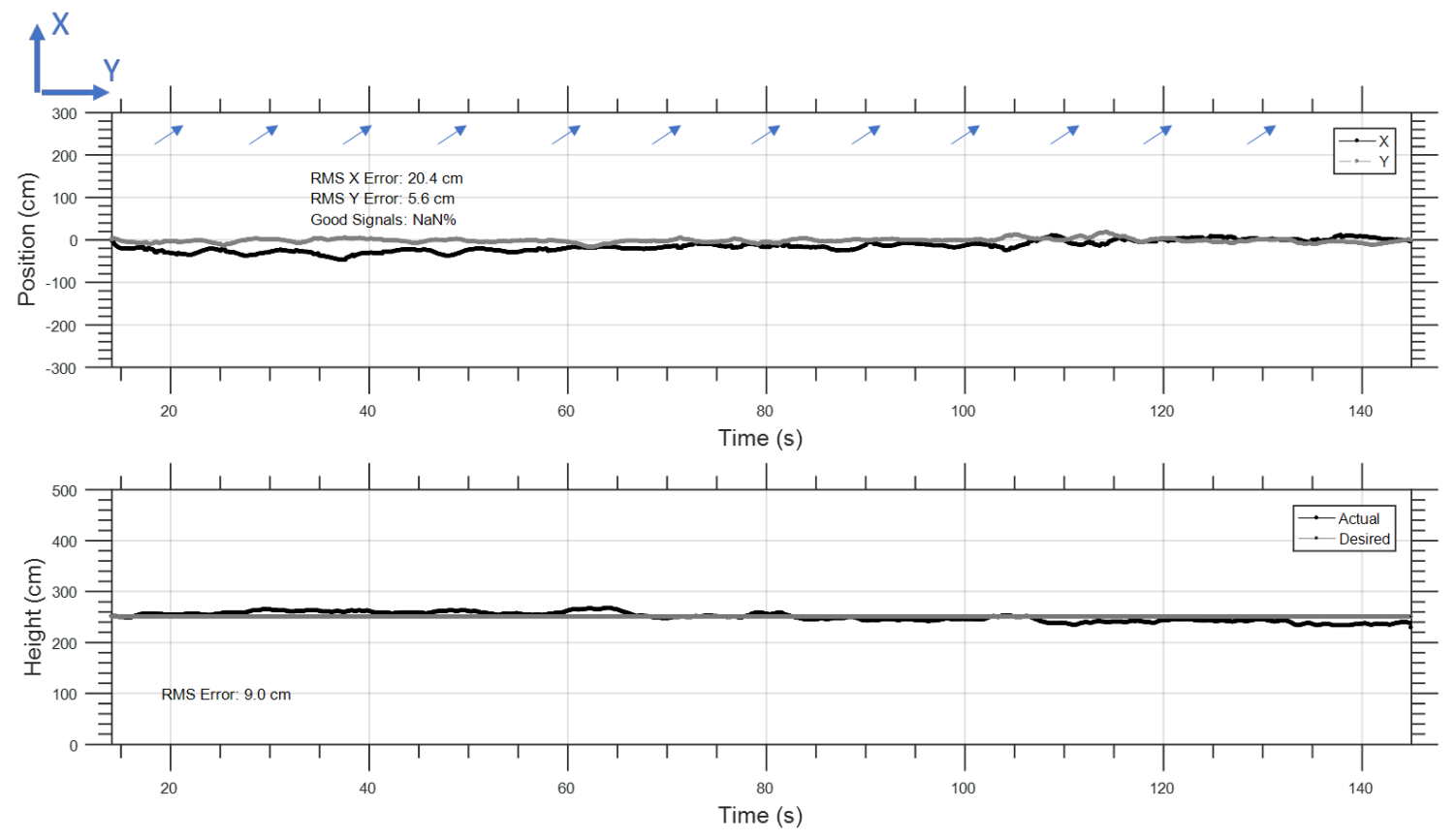

Figure B.2: Hover in an outdoor environment using GPS

to where the acceptable radius lines are shown as red dotted lines in the position graph. At $72 \mathrm{~s}$ TARA 3 briefly enters the acceptable radius, as this happens the desired altitude gets updated to the current altitude of $2.0 \mathrm{~m}$ as shown in the altitude graph at $72 \mathrm{~s}$. Due to poor altitude control it is already at this height and did not need to descend to obtain the new setpoint. Now TARA 3 tries to position over top of the beacon at this new desired altitude of $2.0 \mathrm{~m}$ and reaches the acceptable radius at $84 \mathrm{~s}$ and TARA 3 begins to descend for $2 \mathrm{~s}$ when again it leaves the acceptable radius. However the downward momentum of TARA 3 brings it to the final descent altitude as shown by the blue dash dot line. TARA 3 remains in the final descent region and lands. The green dash double dot line shows the sonar final minimum height measured by the sonar, beyond this there are no more height values. The final measured distance away is $53 \mathrm{~cm}$ (66\% wingspan) from the beacon measured by a tape measure as compared to $18 \mathrm{~cm}$ indoors. This landing took significantly longer than the indoor landing with $78 \mathrm{~s}$ vs $7.5 \mathrm{~s}$. The RMS error along the $\mathrm{X}$ axis is 90.0 
$\mathrm{cm}$ and along the $\mathrm{Y}$ axis is $87.7 \mathrm{~cm}$ which is worse than the indoor performance. The poor performance can be related to poor altitude measurements. This also has worse RMS accuracy than the outdoor hover performance (Fig. B.1) most likely because it did not run as long as the hover which provided time to stabilize over the beacon and decrease the RMS error. The percentage of successful measurements is $81 \%$ which is similar to the indoor performance (Fig. 5.16 86\%) and the outdoor hover (Fig. B.1 $82 \%)$

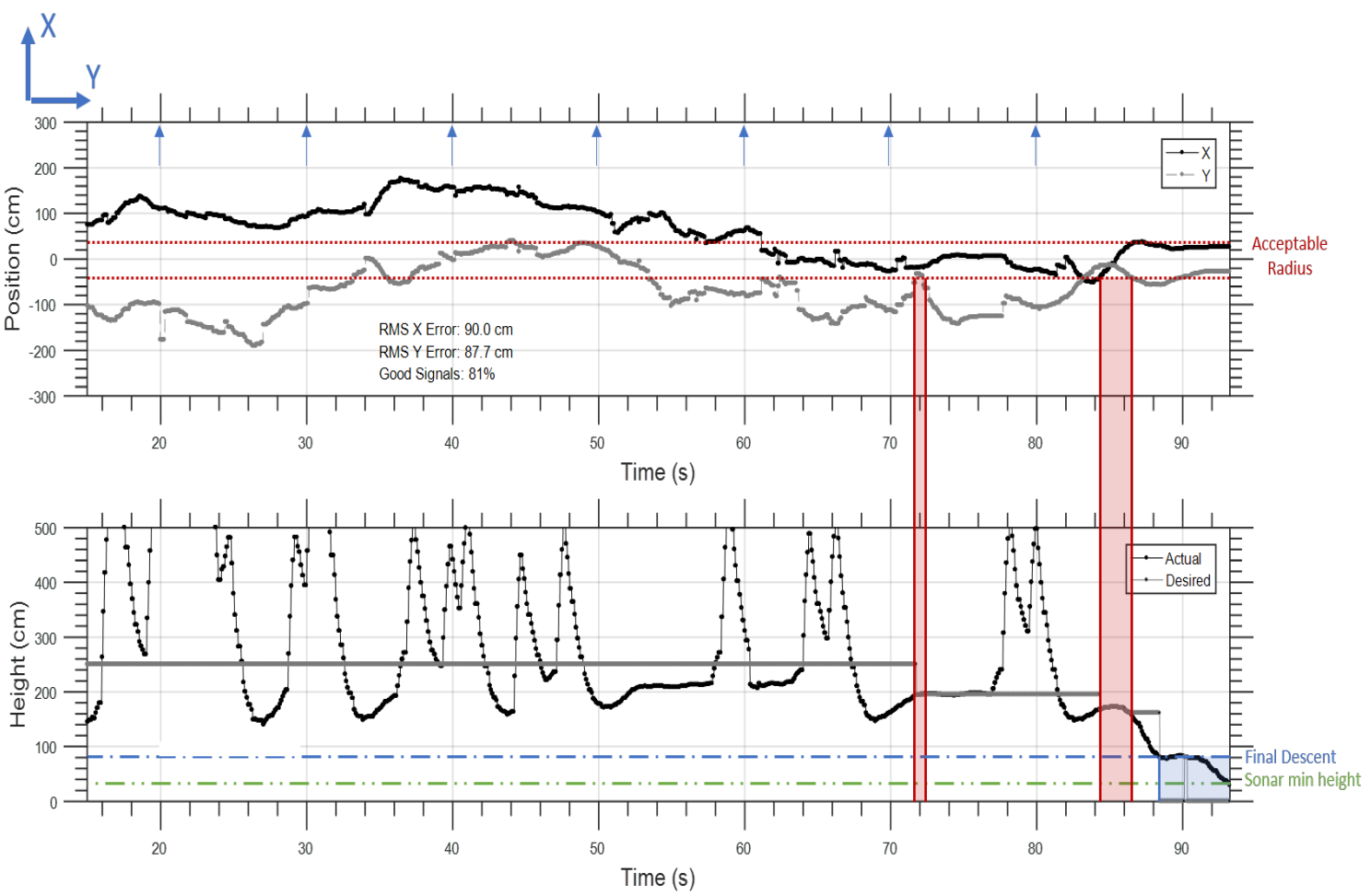

Figure B.3: Landing in an outdoor environment using the ULS

For the GPS system, TARA 3 is manually positioned over the desired coordinate and switched to landing mode which is covered in Section 3.2.3. The desired coordinate is the position directly under TARA 3 when it is switched to land mode $(0,0,0)$. The performance of the GPS landing is shown in Fig. B.4. During the landing there is a RMS error of $17.8 \mathrm{~cm}$ along the $\mathrm{X}$ axis and a RMS error of $7.0 \mathrm{~cm}$ along the $\mathrm{Y}$ 
axis. This RMS is significantly lower than the error from the ULS showing that it has superior performance since it is combined with other measurements through the EKF.
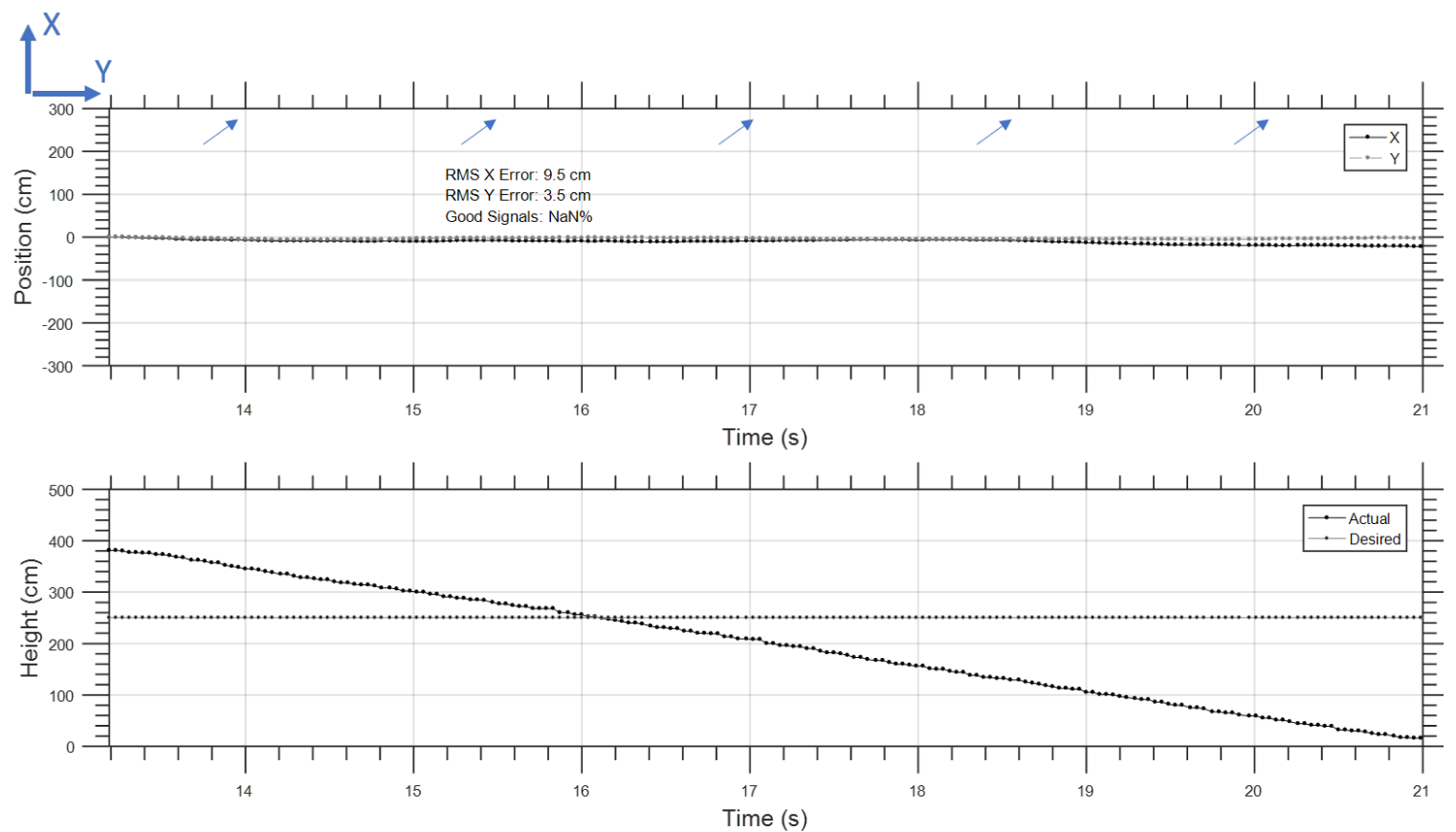

Figure B.4: Landing in an outdoor environment using the GPS system

\section{B.3 GPS ULS Combined Mission}

A package delivery mission profile is tested to see the capability and performance using a precision landing versus the standard performance. The mission consists of a cruise to the beacon using the GPS to control through ArduCopter waypoint navigation. Then switches to land mode to begin descending while maintaining the current coordinate if it is outside the detection range of the ULS. Once an ultrasonic signal is received the ULS takes over control using the Guided No GPS mode to complete a precision landing. For comparison with only GPS, the quadrotor simply 
lands using the land mode at the desired coordinate. The mission profile is shown in Fig. B.5. To estimate the beacon GPS coordinates, TARA 3 is placed stationary at the location of the beacon and the GPS position is averaged over five minutes before the flights.

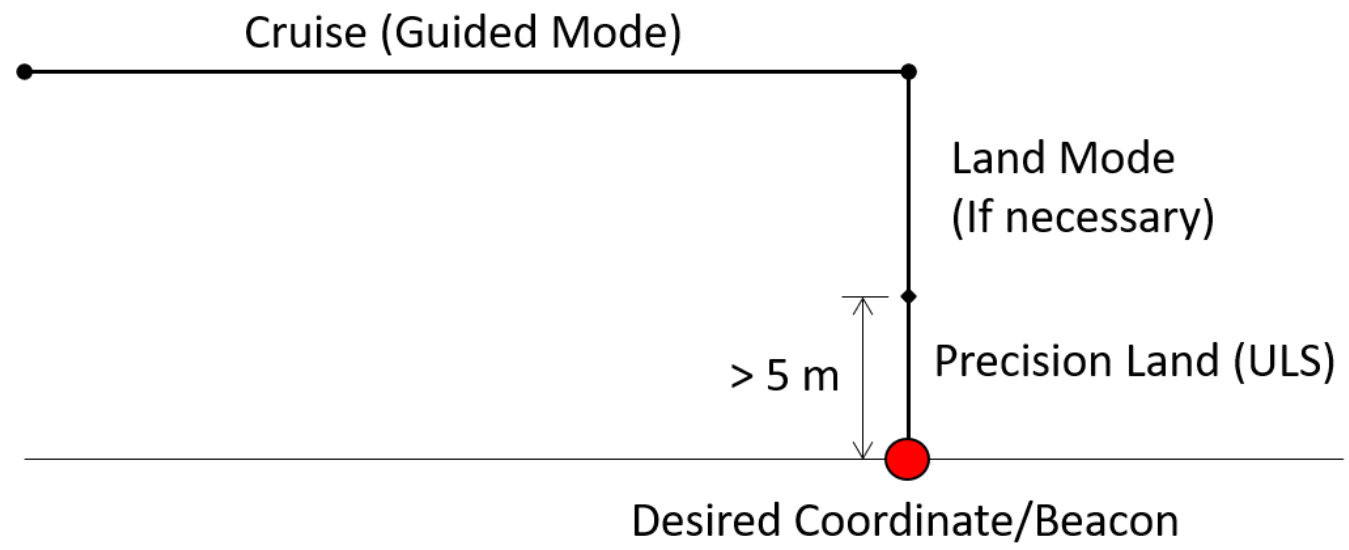

Figure B.5: Mission profile

The landing phase for a mission using the ULS is shown in Fig. B.6. TARA 3 is positioned manually to a position outside of the ULS range and is switched to autonomous mode where the figure begins at 65 s. First TARA 3 uses GPS to control to the beacon as shown by the grey shaded region. The bottom graph shows TARA 3 is hovering at a constant altitude of $4.5 \mathrm{~m}$ towards the beacon. At $79 \mathrm{~s}$ TARA 3 is in range of the ULS and it takes over position control, where it tries to hover to within the acceptable radius. It tries to maintain the current desired height (shown by the dashed line) but the sonar has a difficult time measuring the height accurately due to measurement noise over grass. As the system jumps from GPS positioning to ULS positioning the position jumps because there is a difference between the absolute positioning of the GPS and the ULS. This difference is due to GPS drift after the beacon location $(0,0)$ was calibrated. At $96 \mathrm{~s}$ TARA 3 is within the acceptable range $(40 \mathrm{~cm})$ of the beacon and begins to descend as shown by the green shaded region. 
Once it begins to descend the desired position gets set to the desired position for hover or landing of $2.5 \mathrm{~m}$. It begins to descend but due to noise the height increases mid way through and at $98 \mathrm{~s}$ TARA 3 falls outside of the acceptable radius. It continues to control towards the beacon laterally and tries controlling to a height of $2.5 \mathrm{~m}$. At $102 \mathrm{~s}$ TARA 3 is within the acceptable radius again and begins to descend until 104 s. It falls outside the radius but the momentum keeps it descending. At $105 \mathrm{~s}$ TARA 3 is within the acceptable radius and begins to descend where at $108 \mathrm{~s}$ it reaches the final descent phase $(z<80 \mathrm{~cm})$ as shown with purple until it finally lands. The final position away from the beacon is $32 \mathrm{~cm}$ measured by a tape measure. This shows that the ULS can successfully be combined with ArduCopter waypoint navigation for a precision landing at a beacon.
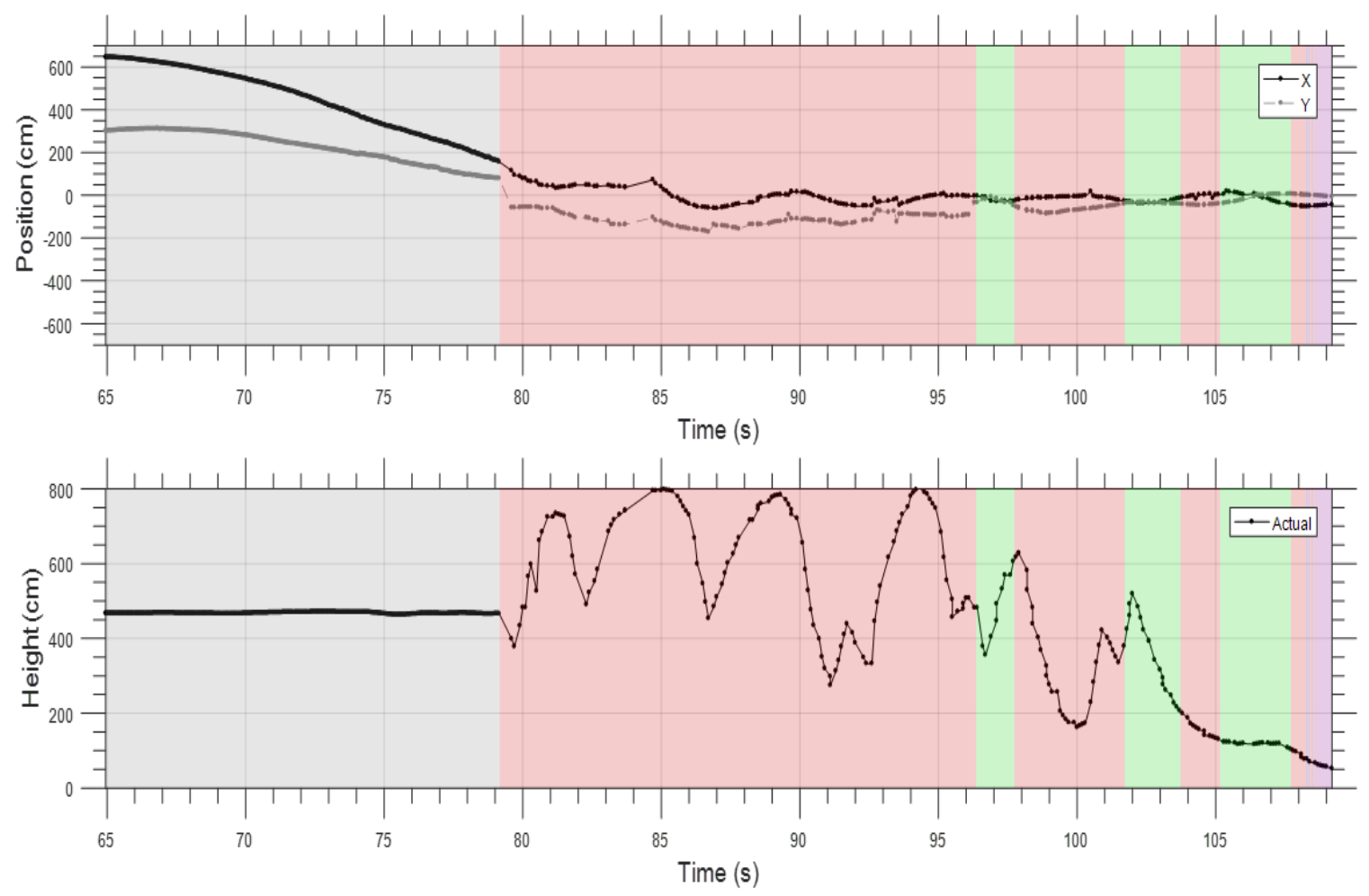

Figure B.6: Mission in an outdoor environment using the ULS

The mission using only GPS follows the same principle except it continues to land 
using GPS to the desired coordinate. During this mission TARA 3 starts a similar distance away and travels to the beacon as shown by the grey shaded region. At 30 s TARA 3 reaches the desired location at a height of $4 \mathrm{~m}$ and starts to descend. It descends and lands at $39 \mathrm{~s}$, according to the GPS it lands at a distance of $3.6 \mathrm{~cm}$ away. However measuring the distance using a tape measure between where TARA 3 lands and the beacon is $195 \mathrm{~cm}$, the absolute location of the GPS is off. The ULS landed with a position $84 \%$ closer than the GPS system alone, it provides an absolute positioning reference which can correct for the GPS drift.
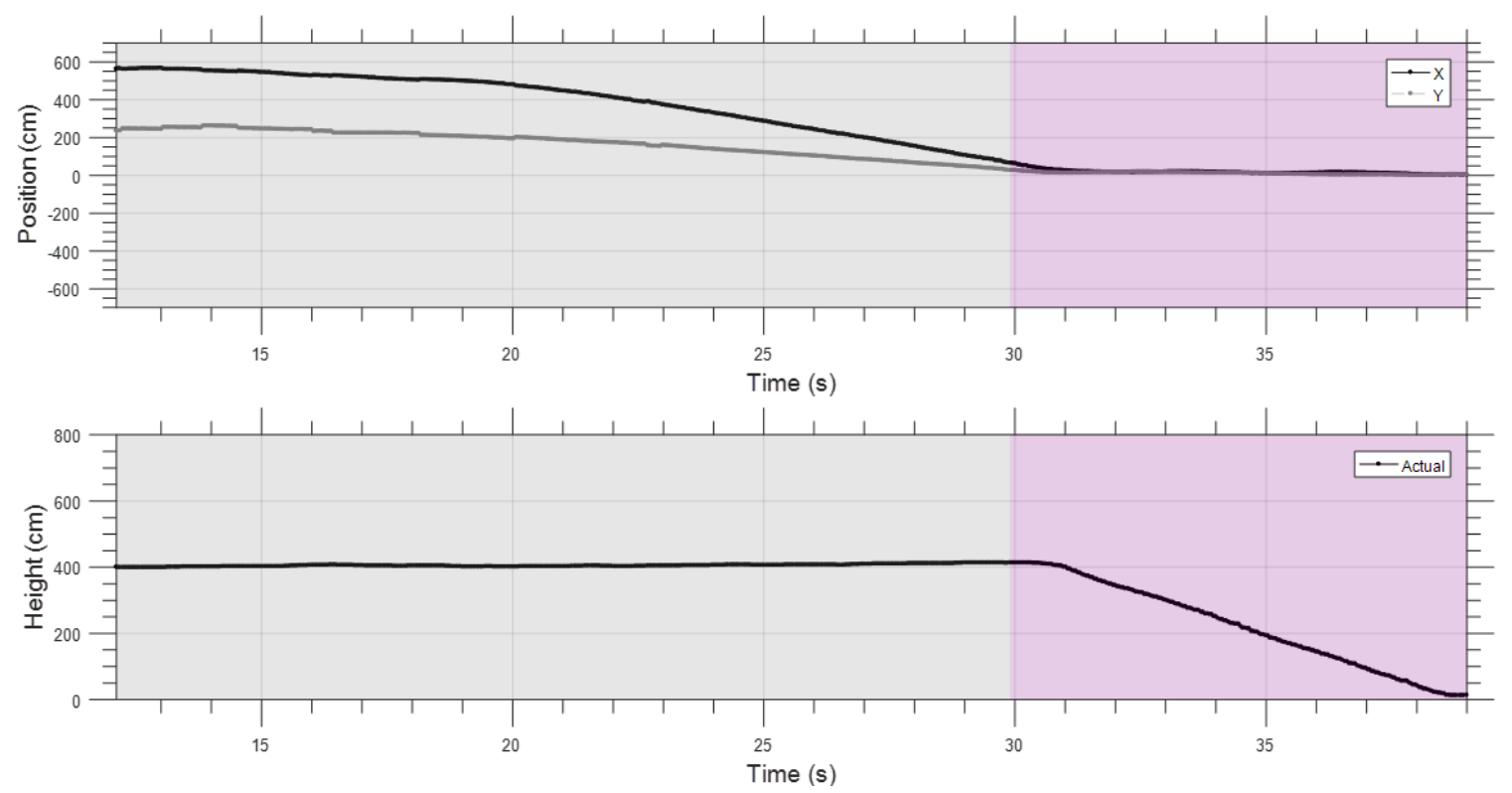

Figure B.7: Mission in an outdoor environment using the GPS 


\section{Appendix $\mathrm{C}$}

\section{Wake Velocity}

In theory, the propeller wake velocity can have an impact on the net propagation of the ultrasonic signal. Based on momentum theory, the wake velocity through the rotor can be related to the thrust by Eq. C.1 [73].The thrust is equivalent to a quarter of the weight of the quadrotor for hover shown by Eq. C.2. Table C.1 summarizes the calculation. The hover velocity is calculated to be $6.29 \mathrm{~m} / \mathrm{s}$ which is less than $2 \%$ of the speed of sound and is considered negligible to the propagation of the net velocity of the sound wave.

$$
\begin{gathered}
v_{r}=\sqrt{\frac{T}{2 \rho A}} \\
T_{\text {Hov }}=0.25 \mathrm{~m}
\end{gathered}
$$


Table C.1: Rotor velocity calculation

\begin{tabular}{|c|c|c|c|}
\hline Parameter & Value & Units & Source \\
\hline \hline Mass & 2.22 & $\mathrm{~kg}$ & Measured \\
\hline Weight & 21.8 & $\mathrm{~N}$ & Calculated \\
\hline Density & 1.13 & $\mathrm{~kg} / \mathrm{m}^{3}$ & Sea Level \\
\hline Radius & 0.140 & $\mathrm{~m}$ & Design \\
\hline Area & 0.0613 & $\mathrm{~m}^{2}$ & Design \\
\hline Hover Rotor Thrust & 5.45 & $\mathrm{~N}$ & Eq. C.2 \\
\hline Hover Rotor Velocity & 6.29 & $\mathrm{~m} / \mathrm{s}$ & Eq. C.1 \\
\hline
\end{tabular}

